\title{
Geodetic investigation of triggered slip below Fiordland
}

By

Danielle M. P. Lindsay

\begin{abstract}
A thesis
submitted to Victoria University of Wellington in fulfilment of the requirements for the degree of Master of Science in Geophysics.
\end{abstract}

Victoria University of Wellington

2019 
"InSAR... allows one the luxury of going on geodetic fishing trips, whereby one essentially asks 'I wonder if.. .?', in search of the unexpected"

- Simons and Rosen (2015) 


\section{Abstract}

Secretary Island, at the head of Doubtful Sound in Fiordland, has been seismically active in past 30 years, with earthquakes larger than $M_{w} 6.5$ : the 1989 Doubtful Sound, 1993 Secretary Island, and 2003 Fiordland earthquakes. These events were approximately coincident with the $17^{\circ}$ bend in the strike of the young, obliquelyconverging, and steeply dipping Puysegur Subduction Zone. This section of the plate interface also has a history of triggered slip: the 1989 earthquake is inferred to have triggered the 1993 earthquake and, further north at George Sound, triggered afterslip was reported following the 2009 Dusky Sound earthquake. We have used L-band (23.6 cm-wavelength) Synthetic Aperture Radar (SAR) data from the ALOS1 and ALOS2 satellites, and C-band (5.5 cm-wavelength) SAR data from Sentinel 1A/B satellites, to test the hypothesis that triggered slip also occurred in the vicinity of Secretary Island following the 2007 George Sound, 2009 Dusky Sound and 2016 Kaikōura earthquakes. SAR images were aligned, interfered, filtered, and unwrapped using GMTSAR processing tools. Long-wavelength ionosphere noise was removed by inverting for the best-fitting linear plane, and we assumed a linear function of height to remove short-wavelength atmospheric noise. Small Baseline Subset (SBAS) timeseries analysis indicated a localised deformation signal centred on Secretary Island following the Dusky Sound earthquake. A re-analysis was undertaken of the co- and post-seismic deformation caused by the Dusky Sound earthquake so that any surface deformation centred on Secretary Island could be isolated. Campaign and continuous Global Positioning System (GPS) data were simultaneously inverted with co- and post-seismic interferograms using a statistical Bayesian modelling approach to determine the optimal Dusky Sound earthquake source parameters. Limitations arising from orbital drift, the frequency of SAR acquisitions and the observation 
geometry hindered our ability to constrain the timing, magnitude and location of reactivated slip from a source similar to the 2003 Secretary Island earthquake. Our findings indicate that slip was not triggered following either the 2007 George Sound earthquake or 2016 Kaikōura earthquake. However, we cannot rule out triggered slip near Secretary Island following the 2009 Dusky Sound earthquake. Any such slip likely occurred on an area of c. $350 \mathrm{~km}^{2}$ (c. $15 \mathrm{~km}$ updip of the Secretary Island epicentre) with an average slip of 1-3 m, producing motion away from the satellite of c. $25 \mathrm{~mm}$ at Secretary Island. 


\section{Acknowledgements}

I would like to thank my supervisors. I thank Ian Hamling for his patience and guidance as I got my head around all things InSAR. I would like to thank John Townend for all the opportunities he has provided me over the years I've been studying. In particular my first geophysics experience in the Whataroa Valley, and in the last year supporting me to attend an InSAR processing short-course and AGU fall meeting in the US. These opportunities have shaped my life in very meaningful ways and I am very grateful.

Over the course of the year, my office mates have kept morale high and the banter flowing. I would like to thank them for creating a positive and supportive working environment, where we could all share the pain of our battles. I would like to thank the $\mathrm{CKCC}$ for the early afternoon caffeine boosts. I am very appreciative to the people around the world who helped via email, answering questions and sharing scripts, in particular, Sandra Slead and Xihoua (Eric) Xi at Scripps Institution of Oceanography who have been superstars. I would also like to thank Peirre Romant for useful conversations at AGU about tremor in Fiordland and for his willingness to share his data.

I am very grateful for the support of my family for housing and feeding me while I studied. A particular mention goes to my Dad, Graeme, who played a big part in editing this thesis. Finally, I would like to thank my husband Andy, he has gone above and beyond in supporting me in my final stages of this project.

This project was generously supported by EQC and Frank Evison Research Scholarship in Geophysics. Additional funding to support travel to AGU came from a Faculty Strategic Research Grant, and UNAVCO supported my attendance at the 'InSAR Processing and Theory with GMTSAR' short-course in San Diego. 
Many figures in this thesis were produced using Generic Mapping Tools (GMT) from Wessel and Smith (2013) 


\section{Contents}

Abstract $\quad$ v

Acknowledgements $\quad$ vii

Contents $\quad$ ix

List of Figures $\quad$ xii

List of Tables $\quad$ xiii

List of Acronyms $\quad$ XV

1 Introduction $\quad 1$

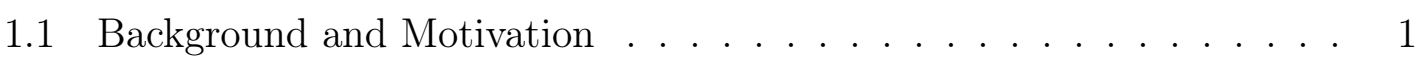

1.2 Tectonic Setting . . . . . . . . . . . . . . . . . 2

1.3 Regional Seismicity . . . . . . . . . . . . . . . . . 7

$1.4 \mathrm{InSAR} \ldots \ldots \ldots \ldots \ldots \ldots \ldots$

1.5 Thesis Objectives . . . . . . . . . . . . . . . . . . 11

1.6 Thesis Structure . . . . . . . . . . . . . . . . . . 12

$\begin{array}{lll}2 & \text { Theory } & 15\end{array}$

2.1 InSAR Background . . . . . . . . . . . . . . . . . . 15

2.2 SAR Acquisition . . . . . . . . . . . . . . . . . 16

2.3 Generating Interferograms . . . . . . . . . . . . . . . . . 22

2.4 Unwrapping Interferograms . . . . . . . . . . . . . . . . . . . . . . . . . . . . . . . . 32

2.5 InSAR Time Series . . . . . . . . . . . . . . . . . . . . . 32

2.6 Incorporation of GPS . . . . . . . . . . . . . . . . . 35

2.7 Modelling . . . . . . . . . . . . . . . . . 36

2.8 Determining Uncertainty in Interferograms . . . . . . . . . . . . . 37

3 Methodology $\quad 41$

3.1 Data . . . . . . . . . . . . . . . . . . 41

3.2 Anticipated Challenges . . . . . . . . . . . . . . . . . . . . 42

3.3 InSAR Processing . . . . . . . . . . . . . . . . . . . . . . . 47

3.4 Short Baseline Subset (SBAS) Time Series . . . . . . . . . . . . . . 67

3.5 Modelling . . . . . . . . . . . . . . . . . . . . . . 69

3.6 Summary . . . . . . . . . . . . . . . . . 76

4 Results $\quad \mathbf{7 9}$

4.1 Preliminary Investigation . . . . . . . . . . . . . . . . . 80

4.2 Interferograms . . . . . . . . . . . . . . . . . . 83 
4.3 Time Series . . . . . . . . . . . . . . . . . . . . . . 87

4.4 Dusky Sound Modelling . . . . . . . . . . . . . . . . . 92

4.5 Isolating Local Deformation Source . . . . . . . . . . . . . . . 97

4.6 Summary . . . . . . . . . . . . . . . . . . . 103

$\begin{array}{llr}5 & \text { Discussion and Conclusions } & 107\end{array}$

5.1 Limitations of the Dataset . . . . . . . . . . . . . . . . 107

5.2 Uncertainty Introduced from Methodology . . . . . . . . . . . . . . . 109

5.3 Comparison of Dusky Sound Deformation Model . . . . . . . . . . . . 111

5.4 Magnitude of Detectable Slip . . . . . . . . . . . . . . . . . . 112

5.5 Triggered Slip near Secretary Island . . . . . . . . . . . . . . . . . . 114

5.6 Comparison with Independent Observations . . . . . . . . . . . . . . 117

5.7 Comparison with Triggered Slip at George Sound . . . . . . . . . . . 118

5.8 Future Research . . . . . . . . . . . . . . . . . . . . . . 120

5.9 Summary and Conclusions . . . . . . . . . . . . . . . . 122

$\begin{array}{ll}\text { Bibliography } & 125\end{array}$

$\begin{array}{ll}\text { Appendices } & 135\end{array}$ 


\section{List of Figures}

1.1 Tectonic setting of Fiordland . . . . . . . . . . . . . . . . . . . 3

1.2 Location of reactivated slip at George Sound . . . . . . . . . . . . . 4

1.3 Cross-section through Puysegur Subduction Zone in Fiordland . . . . 6

1.4 Relocated aftershocks from 1989 and 1993 Fiordland earthquakes . . 10

1.5 Schematic illustrations of the SAR acquisition geometry . . . . . . . . 11

1.6 Schematic of InSAR phase difference . . . . . . . . . . . . . . . . . . 12

2.1 Timeline of InSAR satellite missions . . . . . . . . . . . . . . . . 17

2.2 SAR acquisition geometry and effect of topography on SAR coverage 18

2.3 Schematic of radar chirp function . . . . . . . . . . . . . . . . . . 19

2.4 Zero-Doppler geometry for SLC formation . . . . . . . . . . . . . . 22

2.5 Image geometry showing baseline distance and look angles . . . . . . 23

2.6 Example of interferogram networks . . . . . . . . . . . . . . 27

2.7 Relationship of phase between wrapped and unwrapped images . . . . 29

2.8 Example of closed loop phase residual from Snaphu . . . . . . . . . 29

2.9 Schematic of nearest neighbour interpolation for unwrapping . . . . . 30

2.10 Schematic of linear inversion for a stack of interferograms . . . . . . . 33

2.11 Example comparison of GPS and InSAR derived velocities . . . . . . 39

2.12 Example of InSAR noise field estimation with a semi-variogram . . . 40

3.1 SAR frames used in this study . . . . . . . . . . . . . . . . . . . . . 43

3.2 Baseline distance vs. time plots for ALSO1, ALOS2 and Sentinel 1A/B 44

3.3 Median annual total rainfall for Southland . . . . . . . . . . . . . . 46

3.4 GMTSAR InSAR processing flow . . . . . . . . . . . . . . . . . . 48

3.5 Example's of GMTSAR processing output . . . . . . . . . . . . . . . . 49

3.6 Alignment of SAR images relative to a super-master . . . . . . . . . . 52

3.7 Comparison of images with persistent scatterers weighting . . . . . 53

3.8 Comparison of coherence from SAR images acquired during summer . 54

3.9 Baseline distance plot of tested interferograms networks . . . . . . . . 55

3.10 Region used to select interferograms with high coherence for Network 457

3.11 Gaussian filter tests with increasing spatial wavelength . . . . . . . . 59

3.12 Coherence threshold testing for pixel unwrapping . . . . . . . . . . 61

3.13 Comparison of tests with and without interpolation prior to unwrapping 64

3.14 Ionosphere and atmosphere noise in unwrapped interferograms . . . . 65

3.15 Unwrapped phase, calculated linear plane and detrended interferogram 65

3.16 Comparison between topography and residual noise . . . . . . . . . . 66

3.17 Stacked interferograms, showing sensitivity to coherence and detrending 68

3.18 Comparison of SBAS results from parameters testing . . . . . . . . . 70

3.19 Quad-tree sampling for Dusky Sound modelling . . . . . . . . . . . . 73

3.20 Variogram for track 639 used to estimate noise . . . . . . . . . . . . . 74

3.21 Example of GBIS convergence . . . . . . . . . . . . . . . . 75 
4.1 Preliminary investigation of ALOS1 interferograms . . . . . . . . . 81

4.2 Preliminary investigation of ALOS2 interferograms . . . . . . . . . 81

4.3 Forward model of 2003 Secretary Island displacement field . . . . . . 82

4.4 Displacement fields from Dusky Sound and Secretary Island EQs . . . 83

4.5 Example interferograms with good, moderate and low coherence . . . 84

4.6 Circle test to determine the amount of uncertainty . . . . . . . . . . . 86

4.7 Temporal Constraint on the timing of triggered slip . . . . . . . . . . 87

4.8 Cumulative deformation field . . . . . . . . . . . . . . . . . 89

4.9 Cumulative deformation field - cross section . . . . . . . . . . . . 90

4.10 Cumulative deformation field - pixel time series . . . . . . . . . . . . 91

4.11 Interferograms of the Dusky Sound earthquake . . . . . . . . . . . . 94

4.12 Convergence and PDF plots for final Dusky Sound model parameters 98

4.13 Joint Probabilities for Dusky Sound (uniform slip) . . . . . . . . . . . 99

4.14 Final Dusky Sound Model . . . . . . . . . . . . . . . . . . . . . . . 100

4.15 Data and Model of GPS horizontal displacement . . . . . . . . . . . . 101

4.16 Isolating Secretary Island deformation signal . . . . . . . . . . . . . . 104

4.17 Cross-sections comparing Secretary Island source models . . . . . . . 105

4.18 Histogram of residual data . . . . . . . . . . . . . . . . . 106

5.1 Comparison of modelling with previous work . . . . . . . . . . . 113

5.2 Tectonic interpretation of Secretary Island deformation signal . . . . 114

5.3 Fiordland velocity field following 2009 Dusky Sound earthquake . . . 116

5.4 Alternative tectonic interpretation from GPS observations . . . . . . 118

5.5 Recently reported tremor in Fiordland . . . . . . . . . . . . . . . . . 119

5.6 Recent Seismicity and Tremor . . . . . . . . . . . . . . . . . . . 121

A1 Alignment of SAR images for Sentinel 1A/B . . . . . . . . . . . 137

A2 Alignment of SAR images relative to a super-master . . . . . . . . . . 138

A3 Interferogram Network . . . . . . . . . . . . . . . . . . . . 139

A4 Preliminary investigation - ALOS1 Wrapped phase . . . . . . . . . 140

A5 Preliminary investigation - ALOS2 Wrapped phase . . . . . . . . . . 140

A6 Examples of unwrapping errors . . . . . . . . . . . . . . . . . 141

A7 Example of coherence plots . . . . . . . . . . . . . . . . . . . . 142

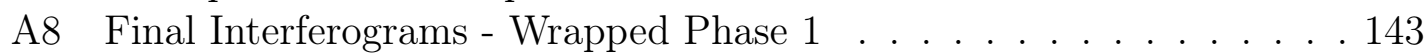

A9 Final Interferograms - Wrapped Phase 2 . . . . . . . . . . . . . . . . 144

A10 Final Interferograms - Wrapped Phase 3 . . . . . . . . . . . . . . . . 145

A11 Final Interferograms - Wrapped Phase 4 . . . . . . . . . . . . . . 146

A12 Final Interferograms - Wrapped Phase 5 . . . . . . . . . . . . . . . . 147

A13 Final Interferograms - Unwrapped Phase 1 . . . . . . . . . . . . . . . 148

A14 Final Interferograms - Unwrapped Phase 2 . . . . . . . . . . . . . . . 149

A15 Final Interferograms - Unwrapped Phase 3 . . . . . . . . . . . . . 150

A16 Final Interferograms - Unwrapped Phase 4 . . . . . . . . . . . . . 151

A17 Final Interferograms - Unwrapped Phase 5 . . . . . . . . . . . . 152 


\section{List of Tables}

3.1 Summary of data sources . . . . . . . . . . . . . . . . . . . . . 42

4.1 Uniform slip plane inversion results . . . . . . . . . . . . . . . . 95

1 Testing of cross correlation parameters for SAR alignment . . . . . 137 


\title{
List of Acronyms
}

\author{
ALOS Advanced Land Observing Satellite \\ CC cross-correlation \\ cGPS continuous Global Positioning System \\ DEM Digital Elevation Models \\ Envisat European Space Agency environmental satellite \\ ERS European Remote Sensing \\ ESA European Space Agency \\ FBD Fine Beam Dual Polarization \\ FBS Fine Beam Single Polarization \\ FFT fast Fourier transform \\ GBIS Geodetic Bayesian Inversion Software V1.0 \\ GMT Generic Mapping Tools \\ GPS Global Positioning System \\ InSAR Interferometric Synthetic Aperture Radar \\ ISCE InSAR Scientific Computing Environment \\ JAXA Japan Aerospace Exploration Agency \\ LOS line-of-sight \\ MAVL Mavora Lakes \\ MCMC Markov Chain Monte Carlo Method \\ PALSAR Phased Array type L-band Synthetic Aperture Radar \\ PDF probability density functions \\ PS Persistent Scatterers \\ PYGR Puysegur Point \\ RMS root mean square \\ SAR Synthetic Aperture Radar \\ SBAS Small Baseline Subset \\ sGPS semi-continuous Global Positioning System \\ SLC single look complex \\ slipBERI slip BayEsian Regularised Inversion \\ Snaphu Statistical-Cost, Network-Flow Algorithm for Phase Unwrapping \\ SNR signal-to-noise ratio \\ StaMPS Stanford Method for Persistent Scatterers \\ TEC Total Electron Content \\ TOPS Terrain Observation with Progressive Scan
}




\section{Introduction}

\subsection{Background and Motivation}

The Fiordland region of southwest New Zealand has experienced three earthquakes greater than $M_{w} 6.5$ in the past 20 years: the $2003 M_{w} 7.2$ Secretary Island, 2007 $M_{w} 6.8$ George Sound and $2009 M_{w} 7.8$ Dusky Sound earthquakes [Figure 1.1]. The section of the plate boundary that ruptured in the 2006 George Sound earthquake was reactivated following the 2009 Dusky Sound earthquake, releasing moment equivalent to a $M_{w} 6.7$ earthquake [Figure 1.2] (Hamling and Hreinsdóttir, 2016). Two lines of evidence suggest that aseismic slip could also be triggered below Secretary Island following large regional earthquakes. First, following the Dusky Sound earthquake an unusual signal in the GPS velocity field was recorded at Secretary Island. The velocity vector at Secretary Island (site DTJ5) was misoriented with respect to neighbouring stations in Fiordland, showing a deviation from the expected deformation field [Figure 5.3, as discussed in Chapter 5] (Hreinsdóttir, pers. comm., 2018). Second, tremor was detected near Secretary Island following the 2009 Dusky Sound and 2016 Kaikōura earthquakes, which may be indicative of reactivated aseismic slip (Peng et al., 2018, Romanet and Ide, 2019). These observations motivate the hypothesis that slip on a patch similar to the 2003 Secretary Island earthquake was reactivated following large regional earthquakes.

Interferometric Synthetic Aperture Radar (InSAR) is a useful geodetic technique to apply in New Zealand due to its spatial coverage, ability to penetrate through cloud cover and precision of measurements (Massonnet et al., 1993). Due to the remoteness and rugged terrain of Fiordland, there are sparse (c. 10-100 km spacing) geodetic ground-based measurements from continuous GPS (cGPS) and cam- 
paign GPS (sGPS). In comparison, InSAR provides near-continuous spatial coverage with horizontal resolution in the order of decimetres $\left(10^{-1} \mathrm{~m}\right)$, vastly improving the spatial density of measurements available. In this study, we analyse a time-series of interferograms centred on Secretary Island to identify new regions of triggered slip following the 2007 George Sound, 2009 Dusky Sound, and distal 2016 Kaikōura earthquakes. As part of this study, we re-analyse the Dusky Sound earthquake deformation field with a Bayesian statistical approach so that displacement from the earthquake can be adequately removed from the dataset.

\subsection{Tectonic Setting}

The present-day Australian-Pacific plate boundary through the continent of Zealandia is comprised of three first-order tectonic structures; the Puysegur Subduction Zone to the south, the Alpine Fault along the west side of the Southern Alps and the Hikurangi Subduction Zone off the East Coast of the North Island (Mortimer et al., 2017). The polarity of subduction switches between the two subduction zones, with the Australian plate being subducted below the Pacific in the Puysegur Subduction Zone and the opposite occurring along the Hikurangi Subduction Zone. Plate motion is additionally accommodated along second-order structures such as the Marlborough Fault Zone, that connects the Alpine Fault and the Hikurangi Subduction Zone, and the Taupō Volcanic Zone in the central North Island which accommodates back-arc rifting.

The Alpine Fault and Puysegur Subduction Zone intersect offshore from Fiordland in the southwest corner of the South Island [Figure 1.1]. At this latitude, the Australian and Pacific plates are obliquely converging at $\sim 37 \mathrm{~mm} / \mathrm{yr}$ and the relative plate motion is partitioned between strike-slip motion on the Alpine Fault in the shallow crust and thrust-faulting on the Puysegur subduction interface at depth (Beavan et al., 2010, Demets et al., 2010). The surface trace of the Alpine Fault terminates north of Resolution Ridge and the fault itself merges with the Puysegur subduction interface (Reyners and Webb, 2002, Sutherland et al., 2000). 


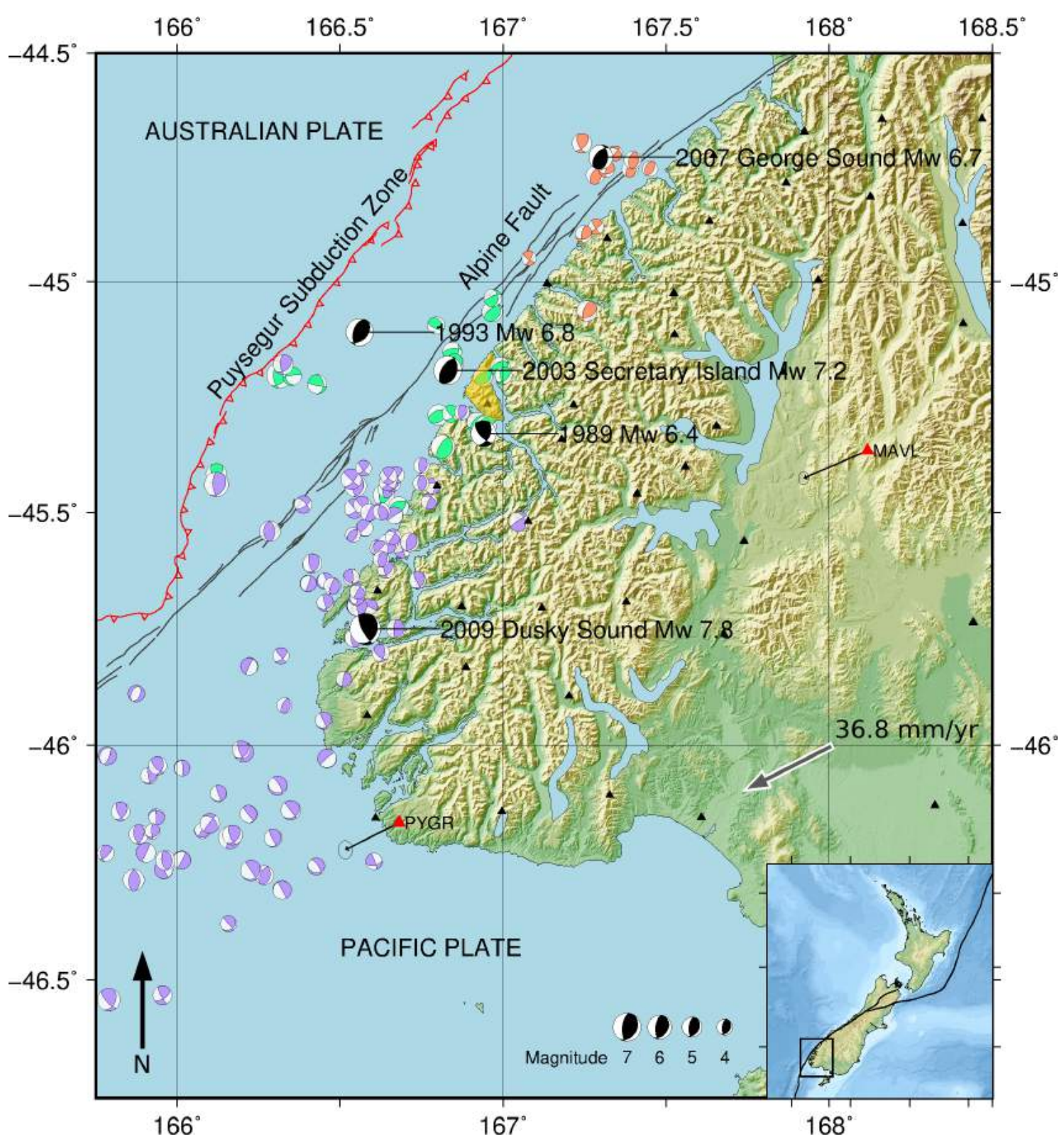

Figure 1.1: Tectonic setting of Fiordland, New Zealand. Plate motion between the Australian and Pacific plates is partitioned between the Alpine Fault(dextral strikeslip) and the Puysegur Subduction Zone (oblique reverse dip-slip). Focal mechanisms for large regional earthquakes and aftershocks from within three months of the 2003 Secretary Island (green), 2007 George Sound (orange) and 2009 Dusky Sound (purple) earthquakes are plotted (Ristau et al., 2016). Continuous (red triangles) and campaign (black triangles) GPS sites are plotted. Grey arrow from MORVEL 200 (Demets et al., 2010) and black arrows at cGPS stations (Beavan et al., 2016) indicate long term plate motion relative to a fixed Australian Plate . Secretary Island has been highlighted in yellow. Faults in black from the Active Fault Database Langridge et al. (2016). 

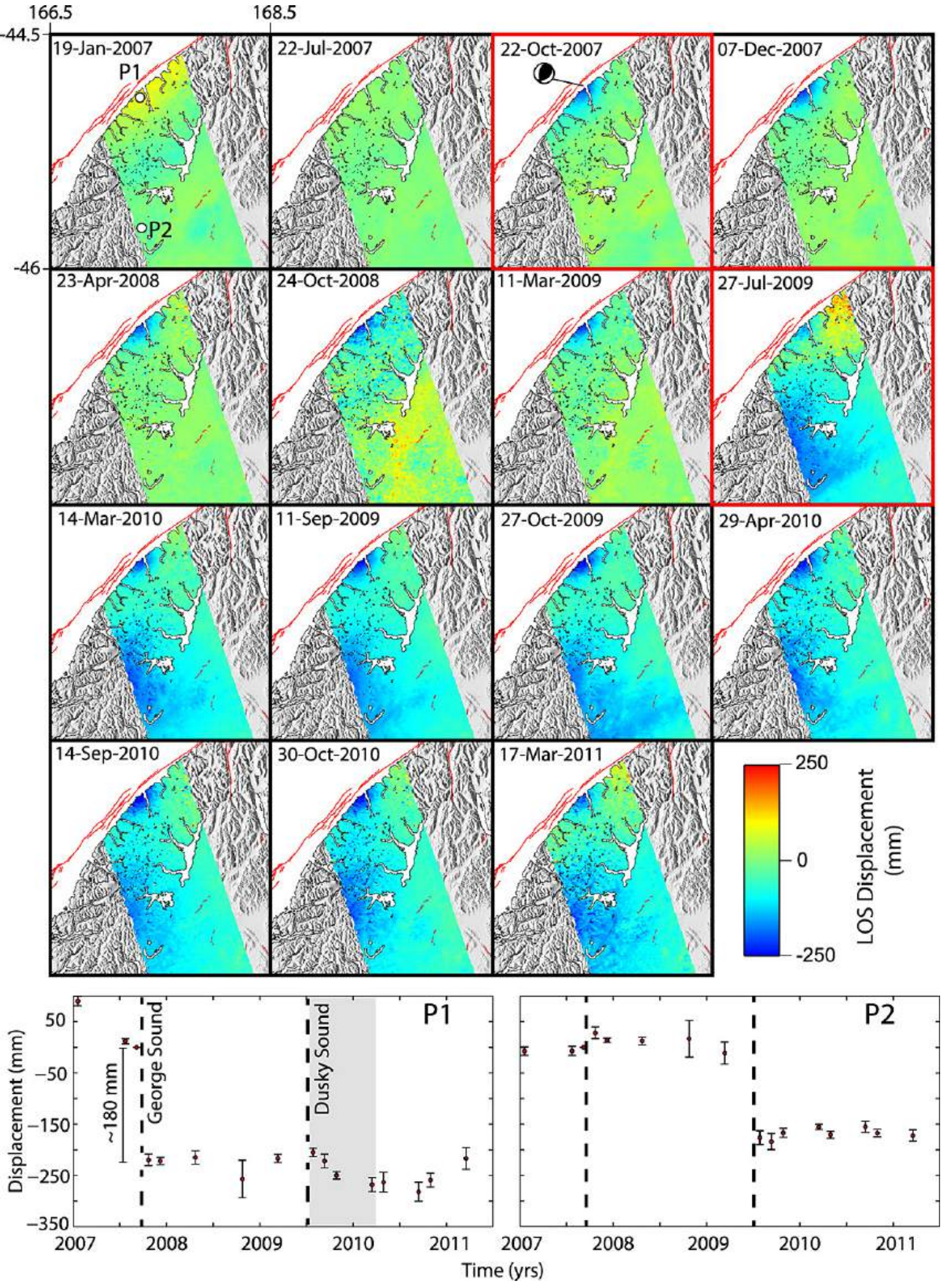

Figure 1.2: (Top) Time series of LOS displacements showing reactivated slip near George Sound, after Hamling and Hreinsdóttir (2016). Red boxes indicate epochs showing deformation associated with 2007 George Sound and 2009 Dusky Sound earthquakes. Negative LOS indicated motion toward the satellite. (Bottom) P1 and P2 (location indicated top left) show clear offsets from the George Sound earthquake, P1 has a curved motion following the Dusky Sound earthquake indicating reactivated slip. 
The Puysegur Subduction Zone formed c. 20 Myr ago as a result of relative changes in motion between the Australian and Pacific plates in the obliquely converging setting (Sutherland, 1995). The Euler pole describing Pacific/Australia relative motion migrated southwards terminating rifting in the Emerald Basin and initiating transform motion along the Alpine Fault (Sutherland, 1995). In the MidMiocene, the inactive Eocene passive margin was subducted below the South Island and it is now located below Fiordland (Sutherland et al., 2000) [Figure 1.3].

The present-day locations of the Alpine Fault and Puysegur Subduction Zone have been suggested to be controlled by the inherited structure of the Eocene passive margin boundary, which separates relatively buoyant Cretaceous continental crust and Eocene-Oligocene oceanic crust (Sutherland et al., 2000). The buoyant continental crust may encourage uplift of the Southern Alps and may have exacerbated the bend in the subducted plate (Eberhart-Phillips and Reyners, 2001). The recent formation of the Puysegur Subduction Zone, in the Miocene, makes it a type example of a young subduction zone that can be studied to investigate subduction initiation processes (Sutherland et al., 2009).

The Puysegur Subduction Zone has an unusually steep dip. At depth, the interface dips steeply eastward at c. $70^{\circ}$ and has a distinct change in strike near Doubtful Sound from $023^{\circ}$ to the south to $040^{\circ}$ to the north (Anderson and Webb, 1994, Eberhart-Phillips and Reyners, 2001, Reyners et al., 2002, WarrenSmith et al., 2017). The Puysegur Subduction Zone has been well imaged with mirco seismicity, with the depth of seismicity increasing in depth from $20 \mathrm{~km}$ below Jackson Bay, to $>100 \mathrm{~km}$ only $40 \mathrm{~km}$ southwest (Warren-Smith et al., 2017). These micro-seismicity observations define a c. $20 \mathrm{~km}$-thick Benioff zone associated with the downgoing Australian plate (Warren-Smith et al., 2017).

The abrupt $17^{\circ}$ change in strike is coincident with the subducting plate approaching a near-vertical orientation as it bends around a high-velocity body $\left(V_{p}>8.5 \mathrm{~km} / \mathrm{s}\right)$ in the mantle (Eberhart-Phillips and Reyners, 2001, Reyners et al., 2002). Sutherland et al. (2000) suggested that the unusually steep bend in the crust has been accommodated by a tear in the Australian Plate. Alternative mantle 


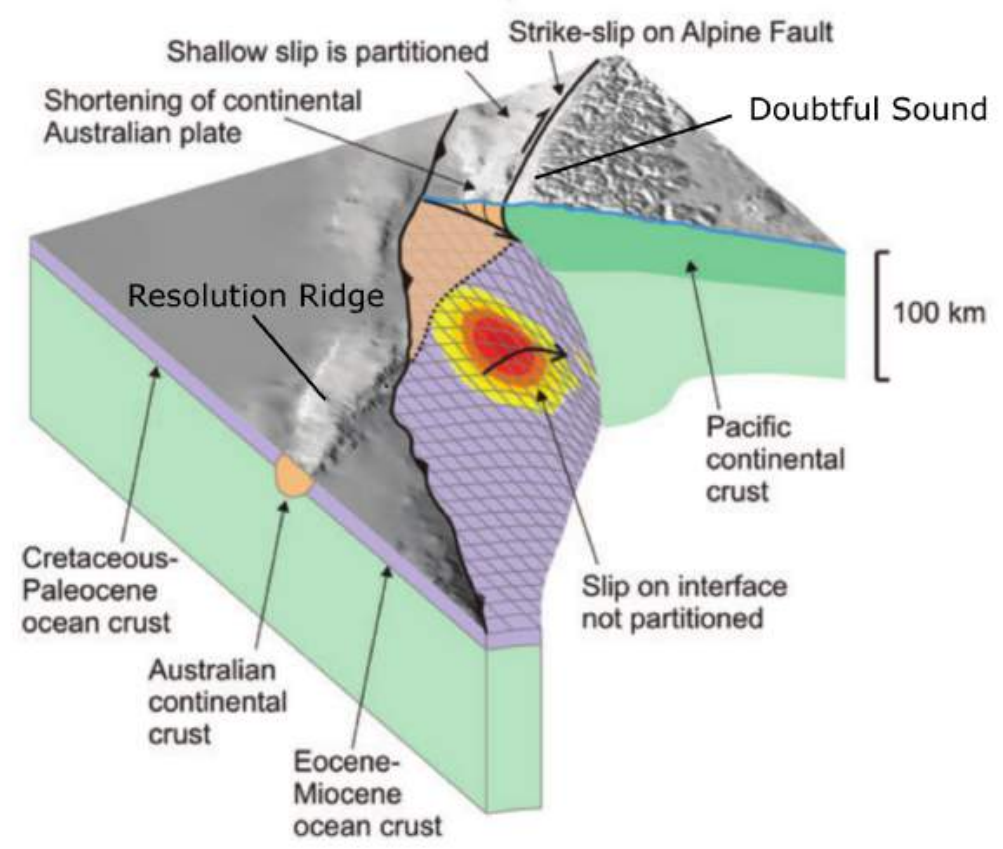

Figure 1.3: Cross-section through Fiordland showing the location of the Eocene passive margin relative to the Puysegur Subduction Zone and the southern portion of the Alpine Fault. The yellow/red shows the modelled rupture region of the 2009 Dusky Sound earthquake, after Beavan et al. (2010).

deformation models of this location include crustal under-plating of the Australian plate forcing the incoming oceanic plate to bend steeply (Lamb, 2015, Warren-Smith et al., 2016), westward subduction of the Cretaceous Hikurangi Plateau (Reyners et al., 2017), and uniform lithospheric thickening producing an anomalous large crustal root (Stern et al., 2000). The lack of data in this region limits the ability to discriminate between proposed deformation models (Boese et al., 2018).

Extensive paleoseismic research reveals that the Alpine Fault is late in its interseismic cycle (e.g. Berryman et al., 2012, Howarth et al., 2018, 2014, 2016) and that the fault is capable of generating earthquakes larger than $M_{w} 8$ (Sutherland et al., 2007). The South Westland portion of the Alpine Fault has a recurrence interval of 291 \pm 23 years and last ruptured in 1717 (Cochran et al., 2017). Strikeslip rates along the Alpine Fault increase southward from $13.6 \pm 1.8 \mathrm{~mm} / \mathrm{yr}$ along the northern section (Langridge et al., 2010), $27 \pm 5 \mathrm{~mm} /$ year through the central section (Norris and Cooper, 2001), increasing to rates of $31.4_{-3.5}^{+2.1} \mathrm{~mm} / \mathrm{yr}$ on the offshore portion of the Alpine Fault (Barnes, 2009). Although there have been several large earthquakes in the Fiordland Region, including 5 larger than $>M_{w} 6.5$ in the past 
30 years, there have not been any earthquakes of this size along the Alpine Fault during the same period.

\subsection{Regional Seismicity}

In the last 30 years, the Fiordland region has experienced a series of moderate to large earthquakes in the upper crust, on the subduction interface and at intraplate depths (Beavan et al., 2010, Doser et al., 1999, Petersen et al., 2009, Reyners et al., 1991, 2003). Here we review seismicity clustered around three locations - Dusky Sound, George Sound and Doubtful Sound - to provide historical context for studying potential new regions of reactivated slip.

The $M_{w}$ 7.8 Dusky Sound earthquake on 15 July 2009 was the largest earthquake to have occurred in New Zealand since the $1931 M_{w} 7.4$ Napier earthquake (Beavan et al., 2010). Slip in this earthquake occurred on the subduction interface below the inferred location of the Eocene rift boundary [Figure 1.3] (Beavan et al., 2010). The focal mechanism of the earthquake indicated predominately oblique motion (Fry et al., 2010). The amount of radiated seismic energy for the Dusky Sound event was relatively low, corresponding to an energy magnitude of $M_{e} 7.2$, that is smaller than the moment magnitude of $M_{w} 7.8$ (Fry et al., 2010). Fry et al. (2010) showed that Coulomb failure stress increased on parts of the southern Alpine Fault following the Dusky Sound earthquake, implying that the Alpine Fault was brought closer to failure (Beavan et al., 2010). Geodetic modelling of the rupture plane by Beavan et al. (2010) indicated maximum slip of c. $5 \mathrm{~m}$ at c. $17 \mathrm{~km}$ depth, $30 \mathrm{~km}$ south-west of the epicentre.

The $M_{w} 6.7$ George Sound earthquake occurred on 16 October 2007. The centroid moment tensor (CMT) solution for the mainshock places the hypocentre at $21 \mathrm{~km}$ depth, c. $20 \mathrm{~km}$ offshore from George Sound with a reverse-faulting mechanism (Petersen et al., 2009). The best-fitting geodetic models of co-seismic displacement and aftershock locations indicated a principal fault plane dipping eastward, which is consistent with rupture of the plate interface (Petersen et al., 2009). Geodetic mod- 
elling of InSAR and Global Positioning System (GPS) data implied $1.7 \mathrm{~m}$ of slip, at c. 16-20 km depth, producing a geodetic moment equivalent to a $M_{w} 6.9$ earthquake (Hamling and Hreinsdóttir, 2016). Two aftershock clusters were observed, indicating activation of a shallow crustal fault, in addition, to slip on the interface (Reyners et al., 2003). Little post-seismic deformation was observed in InSAR timeseries (Hamling and Hreinsdóttir, 2016). Hamling and Hreinsdóttir (2016) used InSAR and GPS to observe the reactivation of the 2007 George Sound fault plane following the 2009 Dusky Sound earthquake. Up to $0.6 \mathrm{~m}$ of afterslip was inferred to have occurred on the plate interface, releasing moment equivalent to a $M_{w} 6.7$ earthquake (Hamling and Hreinsdóttir, 2016).

Doubtful Sound is located between Dusky Sound and George Sound. At the head of the fjord lies Secretary Island, which has been the location of three large earthquakes in the past 30 years: $M_{w} 6.4$ in 1989, $M_{w} 6.8$ in 1993 and $M_{w} 7.2$ in 2003 (Reyners et al., 2003, Reyners and Webb, 2002). The 1993 Secretary Island event ruptured updip of the 1989 Doubtful Sound event and may have been triggered it [Figure 1.4] (Reyners and Webb, 2002). According to this interpretation, the deeper 1989 event may have ruptured an isolated asperity, after which the Coulomb stress on the shallower portion of the interface increased, moving the shallower portion closer towards failure and ultimately triggering the 1993 event (Reyners and Webb, 2002).

On 21 August 2003, a $M_{w} 7.2$ low-angle thrust earthquake ruptured c. $10 \mathrm{~km}$ offshore from Secretary Island, between the 1989 and 1993 epicentres [Figure 1.1] (Reyners et al., 2003). Geodetic measurements from ten sGPS sites were used to model the main shock fault plane and indicated slip on the subduction interface in the direction of subduction (Reyners et al., 2003). Maximum horizontal and vertical displacements of $170 \mathrm{~mm}$ and $130 \mathrm{~mm}$ respectively were recorded near the head of Doubtful Sound (Reyners et al., 2003). The focal mechanisms of all the large events near Secretary Island show predominantly reverse slip geometries with a subordinate dextral component (Reyners and Webb, 2002). Importantly for this study, the findings of Reyners and Webb (2002) addressing the triggering of the 
1993 earthquake sequence by the 1989 earthquake, indicating that the section of the plate interface below Secretary Island has a history of triggered slip.

The Kaikōura earthquake did not occur in Fiordland, but is considered as part of this study as the earthquake triggered slow slip events up the east coast of the of North Island (Wallace et al., 2018) and tremor was detected in the Fiordland region (Peng et al., 2018). On 14 November 2016, the $M_{w} 7.8$ Kaikōura earthquake ruptured 21 faults with a combined rupture length of c.,170 km (Stirling et al., 2017). The complexity of the surface ruptures was revealed with InSAR and GPS, with maximum horizontal displacement of c. $12 \mathrm{~m}$ along the Kekerengu Fault, and maximum vertical displacement of up to $8 \mathrm{~m}$ along the Papatea Fault (Hamling et al., 2017). Shallow slow slip triggered off the east coast of the North Island, and deep slow slip below the southern North Island, released cumulative moment equivalent to a $M_{w} 7.5$ earthquake (Wallace et al., 2018).

\subsection{InSAR}

InSAR is a geodetic technique which calculates the phase difference between two Synthetic Aperture Radar (SAR) images acquired at different points in space and time to produce an interferogram which measures changes in the two-way travel path between the satellite and the ground surface [Figure 1.5, Massonnet and Feigl (1998)]. SAR uses microwave-frequency waves (1 mm-1 m wavelengths) which can propagate through clouds, fog, and smoke without degradation of the signal (Massonnet and Feigl, 1998). The phase difference between SAR acquisitions spanning an earthquake (or other ground-deforming process) provide a measure of the change in path length between the satellite and a point on the earth's surface [Figure 1.6]. Relative surface deformation can be measured with InSAR to millimetre scales, and when used in conjunction with sGPS and cGPS measurements, the absolute value of displacement can also be derived (Bürgmann et al., 2000a).

In this study, we use SAR images acquired by the Advanced Land Observing Satellite (ALOS) 1 and 2 operated by Japan Aerospace Exploration Agency 

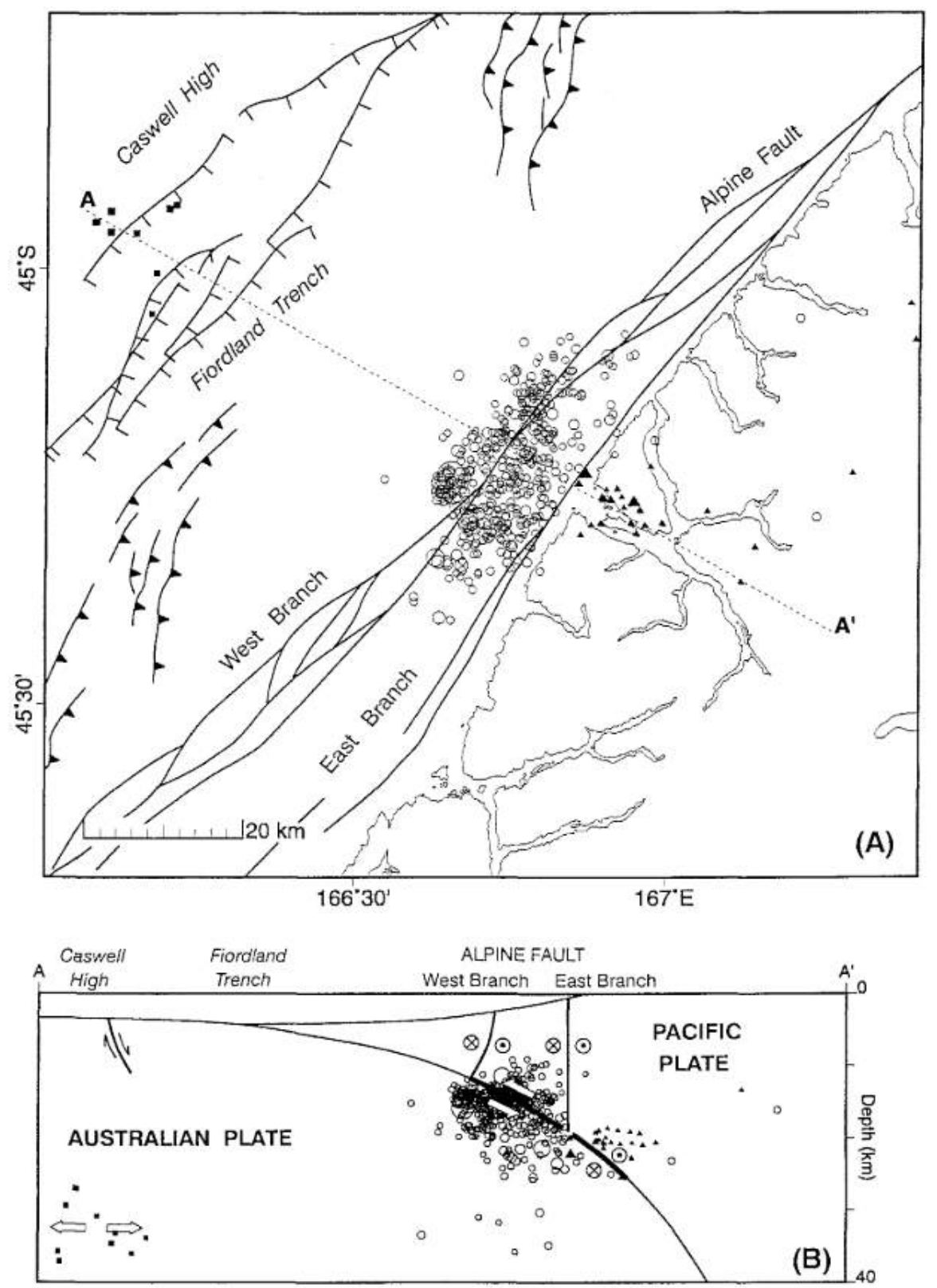

Figure 1.4: Historic earthquakes from Secretary Island (Reyners and Webb, 2002). Triangles represent relocated aftershocks from the 1989 sequence; circles represent thrust events from the 1993 sequence; and squares denote normal events following 1993 sequence. 


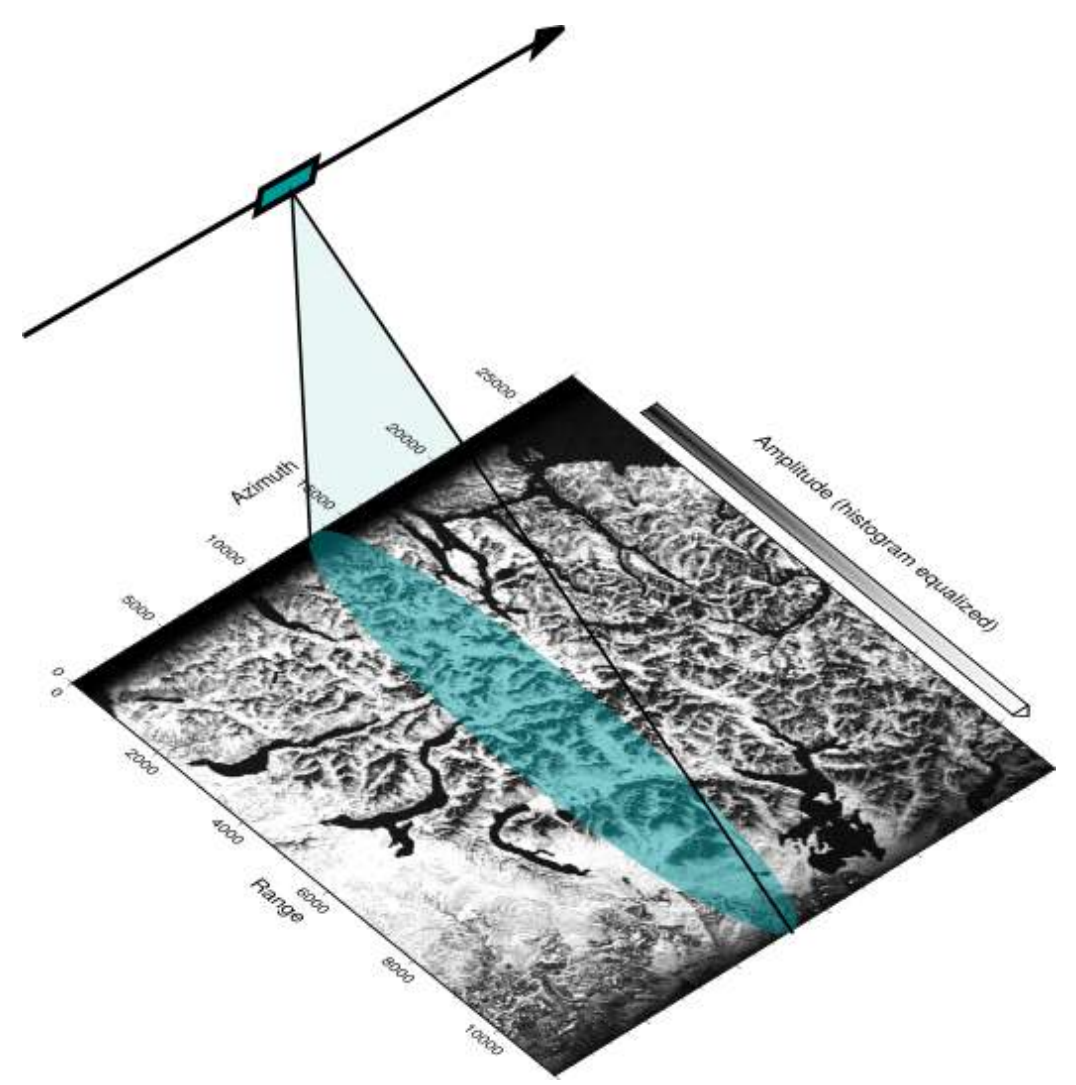

Figure 1.5: Schematic illustrations of the SAR acquisition geometry. Each pulse emitted illuminates an elliptical patch of the Earth's surface from the satellite with a side looking radar. Depending on the scattering properties of the surface of the earth, a proportion of the emitted signal is reflected back to the satellite. The reflected signal provides a measure of the distance between points on the surface of the earth and the satellite.

(JAXA) to resolve deformation between 2006-2011 and 2015-2017, and by the Sentinel 1A/B satellites operated by the European Space Agency (ESA) for 2016-2017. As described in Chapter 3, InSAR processing has been completed using GMTSAR from Sandwell et al. (2011b), Statistical-Cost, Network-Flow Algorithm for Phase Unwrapping (Snaphu) from Chen and Zebker (2000), Small Baseline Subset (SBAS) from Schmidt and Bürgmann (2003) and Geodetic Bayesian Inversion Software V1.0 (GBIS) from Bagnardi and Hooper (2018).

\subsection{Thesis Objectives}

This thesis tests the hypothesis that slip on a patch similar to that which ruptured in the 2003 Secretary Island earthquake was reactivated following the 2007 George 


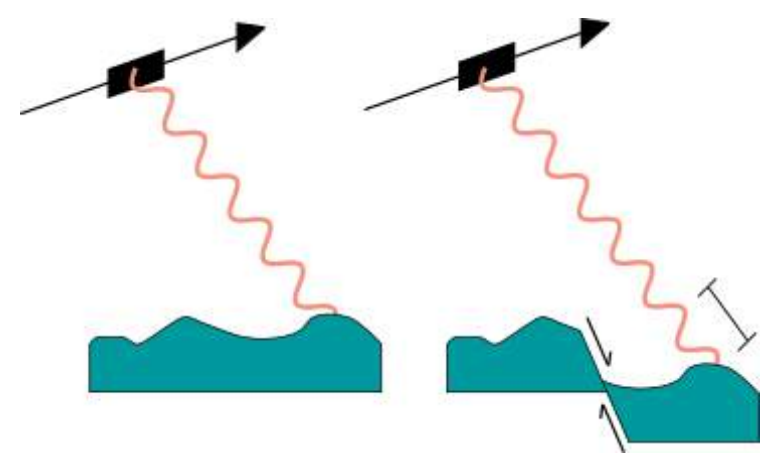

Figure 1.6: Schematic illustration of the phenomenon governing of InSAR phase difference. An earthquake, or other ground deforming event such as a volcanic eruption, landslide or ice stream, shifts the earth away from a satellite making the distance between the two greater. The signal then completes an additional fraction of a cycle and is reflected with a different measure of phase.

Sound, 2009 Dusky Sound and 2016 Kaikōura earthquakes. To address this, we must determine the magnitude of detectable slip and then consider what seismic processes are compatible with the observed deformation field. In the case slip is detected, we will estimate the magnitude, location and temporal constraints of the event. We will address the hypothesis via three specific objectives:

1. Generate interferograms spanning 2007-2011 and 2015-2017 for the purpose of stacking, time series analysis and surface deformation modelling.

2. Complete time-series analysis of interferograms to determine precise postseismic ground deformation following each earthquake.

3. Finally, isolate local deformation centred on Secretary Island to determine the magnitude of any after-slip following the 2007 George Sound, 2009 Dusky Sound or 2016 Kaikōura earthquakes. To do this we must model the coseismic deformation from the 2009 Dusky Sound earthquake for the purpose of accurately removing the deformation field from the data.

\subsection{Thesis Structure}

The structure of this thesis is briefly outlined below with descriptions of the contents of each chapter. This thesis is written in the first personal plural, 'we' rather than 
'I', as this work is being prepared for publication. However, all work in this thesis was completed by Danielle Lindsay. This thesis is structured as follows:

- Chapter 1: An introduction to the tectonic history, recent seismicity of Fiordland and InSAR techniques, and objectives for this project.

- Chapter 2: A review of SAR acquisition, InSAR processing and modelling methods applied in this project.

- Chapter 3: Methods of data processing, parameter testing, time series generation and modelling.

- Chapter 4: Observations and modelling results including preliminary investigation, interferograms, time series of cumulative displacement and Bayesian modelling.

- Chapter 5: Discussion of limitations and uncertainties, the magnitude of detectable slip within the data, and the results presented in Chapter 4, followed by concluding statements. 


\section{Theory}

This chapter describes the theory underpinning Synthetic Aperture Radar (SAR) acquisition and focusing, Interferometric Synthetic Aperture Radar (InSAR) processing, time series analysis and Bayesian modelling. Where applicable, current best practice for InSAR processing is explained and sources of error, error corrections and sources of uncertainty in an InSAR-derived velocity field are discussed.

\subsection{InSAR Background}

The early 1990s heralded a new era in geodesy whereby the application of satellitebased InSAR mapping increased spatial resolution and the precision of measurements to previously unobtainable accuracy (Goldstein et al., 1993, Massonnet et al., 1993). This allowed for the derivation of the complete three-dimensional (3D) displacement field of earthquake, volcano and cryosphere deformation with unprecedented detail (Goldstein et al., 1993, Massonnet et al., 1993). Following the 1992 Landers earthquake in California, the fault rupture and co-seismic displacement were mapped at a resolution of $100 \mathrm{~m}$ to a precision of c. $3 \mathrm{~cm}$ (Massonnet et al., 1993). At a similar time, InSAR was first applied to monitoring ice sheet motion in Antarctica (Goldstein et al., 1993). The detection limit with InSAR for ice stream motion was $4 \mathrm{~mm}$ horizontally and $1.5 \mathrm{~mm}$ vertically, enabling the grounding line of the ice sheet to be mapped with a resolution of $0.5 \mathrm{~km}$ (Goldstein et al., 1993).

SAR uses electromagnetic waves in the microwave spectrum that can penetrate through cloud-cover and operate day and night (Bürgmann et al., 2000a). Early SAR platforms used C-band $(\lambda \approx 6 \mathrm{~cm})$, and L-band $(\lambda \approx 24 \mathrm{~cm})$ (Massonnet and Feigl, 1998). Satellites launched since 2008, such as TerraSAR-X, have also 
included $\mathrm{X}$-band $(\lambda \approx 3 \mathrm{~cm})$ which reflects off urban structures and is useful for engineering applications (Simons and Rosen, 2015).

Early InSAR studies were limited in part by the computational demands of processing large datasets and the number and quality of SAR images acquired (Chen and Zebker, 2000). During the 1990s, four civilian satellites with SAR capacity were in orbit: ERS-1, ERS-2, JERS-1 and RADAR-SAT [Figure 2.1] (Massonnet and Feigl, 1998). Project-specific satellites were launched with orbits designed to capture deformation of specific regions, including RADARSAT-1 which provided extensive coverage of Arctic Ocean sea ice (Massonnet and Feigl, 1998). Since the early 2000s, radars operating at different microwave frequencies have been launched including, the Advanced Land Observing Satellite (ALOS) 1 and 2 (L-band) satellites from Japan Aerospace Exploration Agency (JAXA) and Sentinel 1A and 1B (C-band) satellites from the European Space Agency (ESA) (Rosenqvist et al., 2007, Torres et al., 2012). The NASA-ISRO SAR (NISAR) mission is set to launch in 2021, with radar equipment (for both C-band and L-band) optimised for studying hazards and global environmental change (Rosen et al., 2015). With one satellite, two components of a deformation field can be derived, whereas with two or more satellites the full 3D deformation field can be mapped: as more satellites are launched, global coverage of deformation events continues to improved(Simons and Rosen, 2015).

\subsection{SAR Acquisition}

SAR measures the two-way travel path of a microwave signal between a satellitebased radar and a point on the Earth's surface. As the satellite travels along its orbital track, the ground is illuminated by a side-looking beam and the amplitude and phase of the reflected signal are recorded (Massonnet and Feigl, 1998) [Figure 2.2]. The look angle of the antenna is typically right-looking, meaning that the ascending (south to north) and descending (north to south) passes of the satellite observe the same scene from opposite directions (Simons and Rosen, 2015). More recently deployed satellites, such as ALOS2, have the ability to steer the beam to 


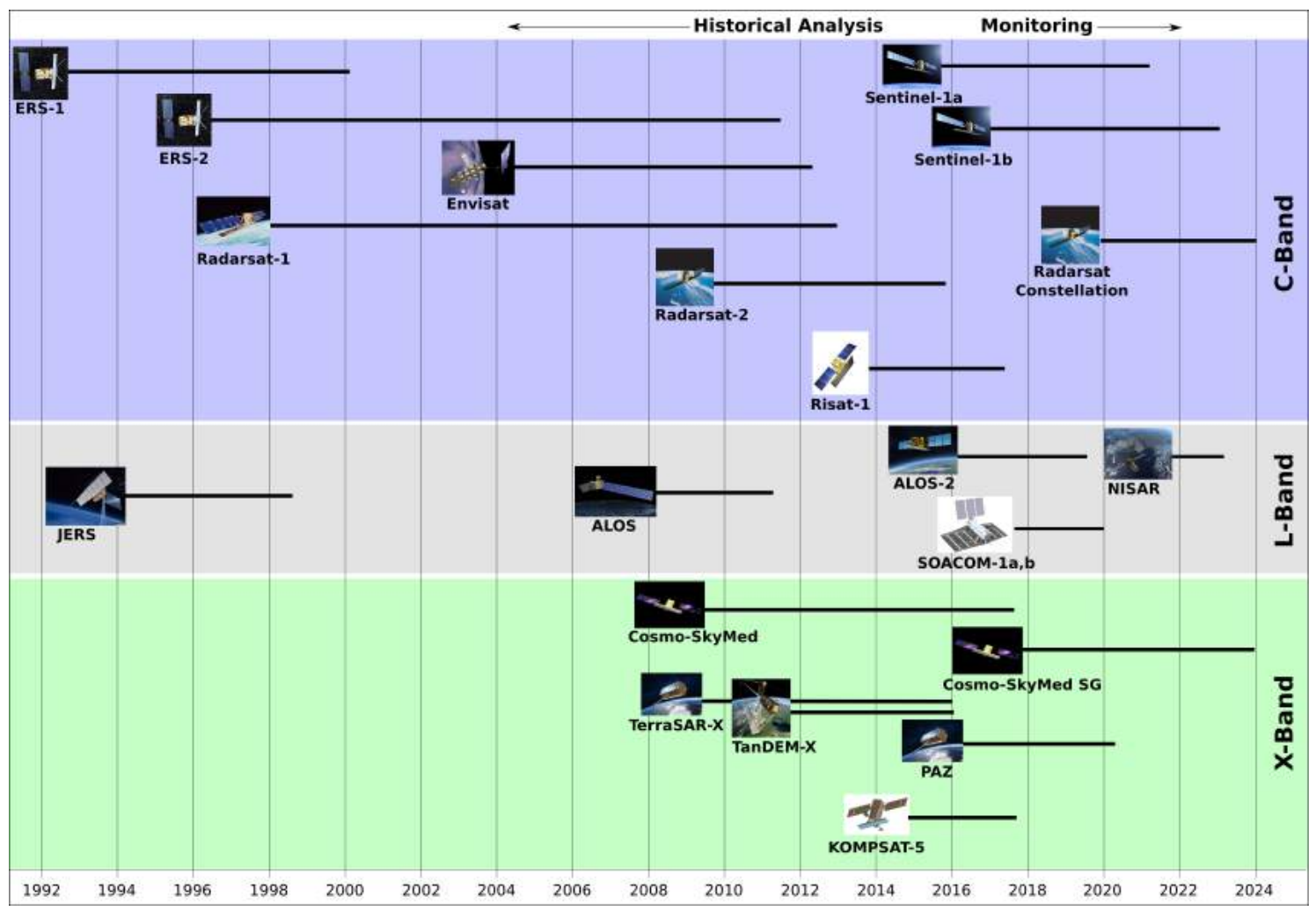

Figure 2.1: Timeline of SAR missions (UNAVCO, 2018). This project uses data from ALOS, ALOS2, Sentinel-1A and Sentinel-1B for the period 2011-2018. Satellite missions are grouped based on their signal wavelngth: C-band $(\lambda \approx 6 \mathrm{~cm})$ which scatters in foliage, and L-band $(\lambda \approx 24 \mathrm{~cm})$ which penetrates to the ground surface and $\mathrm{X}$-band $(\lambda \approx 3 \mathrm{~cm})$ which reflects off urban structures (Massonnet and Feigl, 1998). 


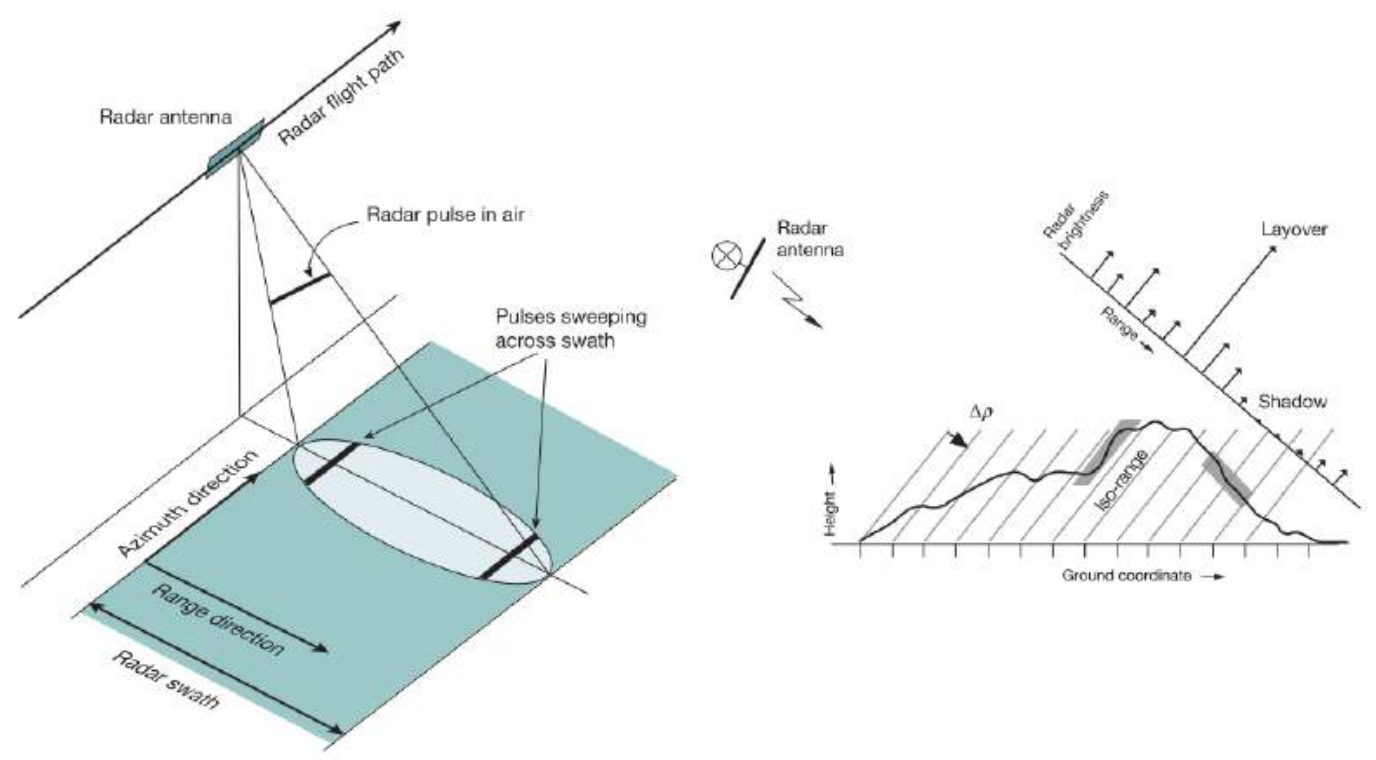

Figure 2.2: (Left) The SAR instrument is carried on a platform which travels along a curvilinear path in the 'along-track' or 'azimuth' direction. The pulse from the radar sweeps across the 'radar swath' in the 'range' direction and the back-scattered signal bounces back to the radar (Simons and Rosen, 2015). (Right) The backscattered energy is collapsed into a $2 \mathrm{D}$ image with range-azimuth coordinates. Note the 'shadow' region forming a data gap on the far side of the hill where the wavefront does not propagate (Simons and Rosen, 2015).

look either left or right in order to observe the same scene from difference orbital geometries (Liang and Fielding, 2017).

The ideal radar pulse is a delta function, as a short pulse-length allows two closely-spaced points on the Earth's surface to be discriminated accurately (Rosen et al., 2000). However, signals with shorter wavelengths require a stronger source to travel from the satellite to earth and back, and recording of the reflected signal must be completed more frequently. To overcome this, the radar pulse is emitted as chirp function that is later compressed with a matched filter to remove the chirp and model the signal as a delta function [Figure 2.3] (Simons and Rosen, 2015).

The transmitted signal has a known amplitude, polarisation, phase, time reference, and frequency (Massonnet and Feigl, 1998). Once the signal is reflected, or scattered by the ground surface, a portion of the signal returns to the radar receiver and the two-way travel time is measured (Simons and Rosen, 2015). Rocks, vegetation, and urban structures are examples of scatterers: they each reflect the energy differently, depending on the wavelength of the incoming radar signal and 


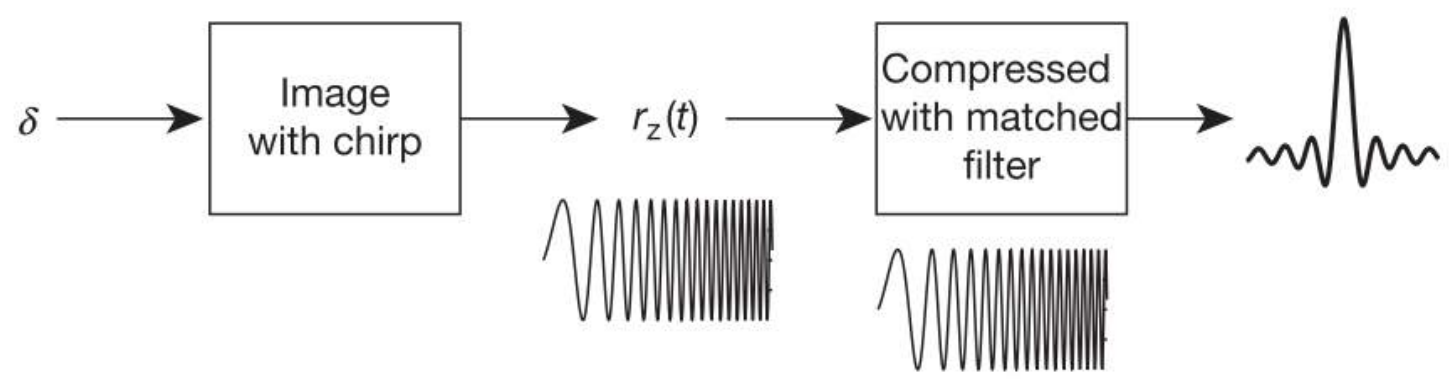

Figure 2.3: The radar emits the signal as a chirp function. During focusing, the image is compressed with a matched filter to devolve the chirp. The radar signal then approximates a delta function and resolution is improved (Simons and Rosen, 2015).

the azimuth and look-angle of the satellite (Ferretti et al., 2007). Each pixel in a SAR image is the sum of all scatterers on the ground within the corresponding ground resolution element. The amplitude of the returned signal is dependent on the amount of scattered energy reflected back to the receiver (Bürgmann et al., 2000a). The returned phase of the signal is a two-way range measurement of the number of cycles completed between the radar and the surface and back again.

There are two modes of data collection used in this study: Strip Map used by ALOS1 and ALOS2, and Terrain Observation with Progressive Scan (TOPS), used by Sentinel. Strip Map records one swath continuously as the radar travels along-track producing a single frame with a width of c. $80 \mathrm{~km}$ (Massonnet and Feigl, 1998). ALOS1 and ALOS2 have two ways of recording Strip Map data: Fine Beam Single Polarization (FBS) and Fine Beam Dual Polarization (FBD), which has a narrower bandwidth than FBS (Liang and Fielding, 2017, Rosenqvist et al., 2007). To process images with different polarisations, they need to be converted to a common polarisation, and best practice is to down-sample the FBD to FBS. TOPS makes three progressive sweeps to generate one swath with a width of c. $250 \mathrm{~km}$ made up of three sub-swaths that can be processed independently or as a single image (Torres et al., 2012). ALOS1 data are available in raw unfocused format, whereas ALOS2 and Sentinel 1A/B data are focused by the associated agency. 


\section{Precise Satellite Orbits}

Precise knowledge of each radar satellite's orbit is very important for correct focusing, transformation from radar to geographic coordinates, alignment of images and removing topographic components from interferograms (Massonnet and Feigl, 1998). Precise orbits are computed frequently in real-time and re-calculated retrospectively with higher precision, so the difference between the expected location and the true location can be corrected for during processing. The precise orbit information is contained in the header of ALOS1 and ALOS2 data and can be downloaded as a separate file for Sentinel 1A/B data.

\section{SAR Image Focus}

A raw SAR image is focused into a single look complex (SLC) format so that phase information at a decimetre resolution (on the ground-surface) can be extracted. The SLC is an image in the range and azimuth imaging plane, in which each pixel contains both amplitude and phase information as a complex magnitude value (Rosen et al., 2000). To produce a SLC, each echo must be focused in the range and azimuth directions, and the successive echos then sorted with respect to the shortest two-way travel-path for each ground point and the satellite along azimuth (i.e. zero-Doppler geometry) (Rosen et al., 2000).

Each pulse emitted by a radar illuminates an elliptical region on the ground surface of approximately $10 \mathrm{~km} \times 80 \mathrm{~km}$ in the range and azimuth directions. The range resolution $\left(R_{r}\right)$ is dependent on the speed of light $(c)$, the pulse length $(\tau)$ and the look angle $(\theta)$ (Bürgmann et al., 2000a):

$$
R_{r}=\frac{c \tau}{2 \sin \theta}
$$

To obtain the required resolution, a succession of individual echos are focused into a SAR image. Range compression deconvolves the range chirp function to sharpen the focusing of the image in the range direction (Rosen et al., 2000). Range migration 
uses the Doppler frequency shift of the returned signal and the precise location of the satellite to flatten the image and make a single feature appear as a straight line in the azimuth direction [Figure 2.4] (Bürgmann et al., 2000a). This is a similar process to that involved in forming a seismic shot gather, whereby a point-source reflector on the ground surface produces a travel-time parabola as the satellite receives echos at different distances along-track in the azimuth direction.

Azimuth compression is then completed to collapse a straight line in the azimuth direction back to the original point source by deconvolving the azimuth chirp (Rosen et al., 2000). The azimuth resolution $\left(R_{a}\right)$ for a single echo is dependent on the the slant angle $\left(\theta_{r}\right)$, height of the satellite $(H)$, nominal slant range $(\rho=$ $H / \cos \theta)$, the wavelength of the signal $(\lambda)$ and the length of the radar $(L)$ (Bürgmann et al., 2000a):

$$
R_{a}=\rho \sin \theta_{r}=\frac{\rho \lambda}{L}
$$

Finally, the successive echos are sorted with regard to the zero-Doppler geometry (Rosen et al., 2000). Echos which fall along the line of equi-Doppler and equidistance are organised relative to each other within the SLC [Figure 2.4] (Massonnet and Feigl, 1998). The zero-Doppler line falls where the range distance between a point on the surface and satellite is the shortest (the near-range distance). When arranging echos in zero-Doppler geometry, each pixel is organised as if it is being viewed from directly above (i.e. the location of shortest-range distance) (Massonnet and Feigl, 1998).

To achieve adequate coverage in generating a SAR image, the footprints of each pulse must overlap, requiring the radar to emit one pulse every half-length of the radar as the satellite travels along orbit (Massonnet and Feigl, 1998). The pulse repetition frequency must be high enough to sample the whole Doppler spectrum to avoid aliasing, and must have sufficient time between pulses so that the far-range return of one pulse is received before the near-range return of next pulse [Figure 2.4] (Rosen et al., 2000). The pulse rate is also limited by the rate at which the data can be transmitted to the ground. For a $10 \mathrm{~m}$-long radar $(L)$ a pulse must be emitted 
Figure 2.4: Geometry of side-looking radar showing geometry of the zero Doppler line. The echos from a radar pulse are sorted with regards to the travel time of the returned sample and the Doppler frequency shift as the radar views a target on the ground from successive echos (Massonnet and Feigl, 1998).

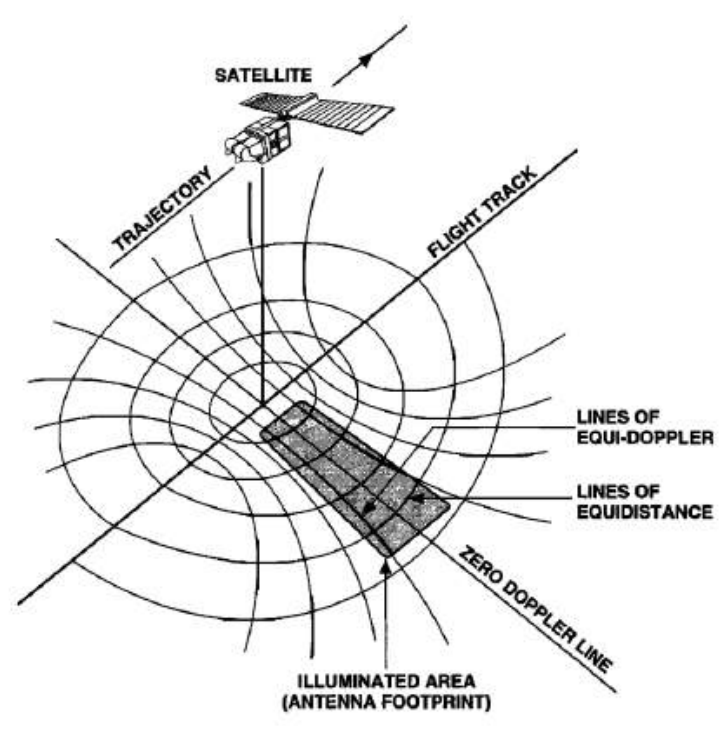

every $5 \mathrm{~m}$, yielding an azimuth resolution $\left(R_{a}^{\prime}\right)$ of $5 \mathrm{~m}$ (Massonnet and Feigl, 1998):

$$
R_{a}^{\prime}=\frac{\lambda H}{2 R_{a} \cos \theta}=\frac{L}{2}
$$

Once the SAR focus has been performed, the satellite has orbited the Earth emitting chirps at microwave frequencies, the returning phase and amplitude information reflecting off the earth's surface was recorded, the returned echoes are deconvolved and sorted so the SLC image appears as if the whole scene was observed from directly above at with a decimetre resolution.

\subsection{Generating Interferograms}

The interference pattern is extracted from two SLC images by multiplying, pixel by pixel, the phase of the first image $\left(\phi_{1}\right)$ by the complex conjugate of the phase of the second image $\left(\phi_{2}\right)$ (Massonnet and Feigl, 1998). The phase measurement of the SLC image $\left(\phi_{1}, \phi_{2}\right)$ represents the path length between the satellite and the ground surface $\left(\rho_{1}, \rho_{2}\right)$ [Figure 2.5]. In calculating the interferometric phase $\left(\phi_{I}\right)$, the common phase component in each image cancels leaving only the phase difference due to changes in the differential path delay $(\delta \rho)$ (Zebker et al., 1994):

$$
\phi_{I}=\phi_{1}-\phi_{2}=\frac{4 \pi}{\lambda}\left(\rho_{2}-\rho_{1}\right)=\frac{4 \pi}{\lambda} \delta \rho
$$




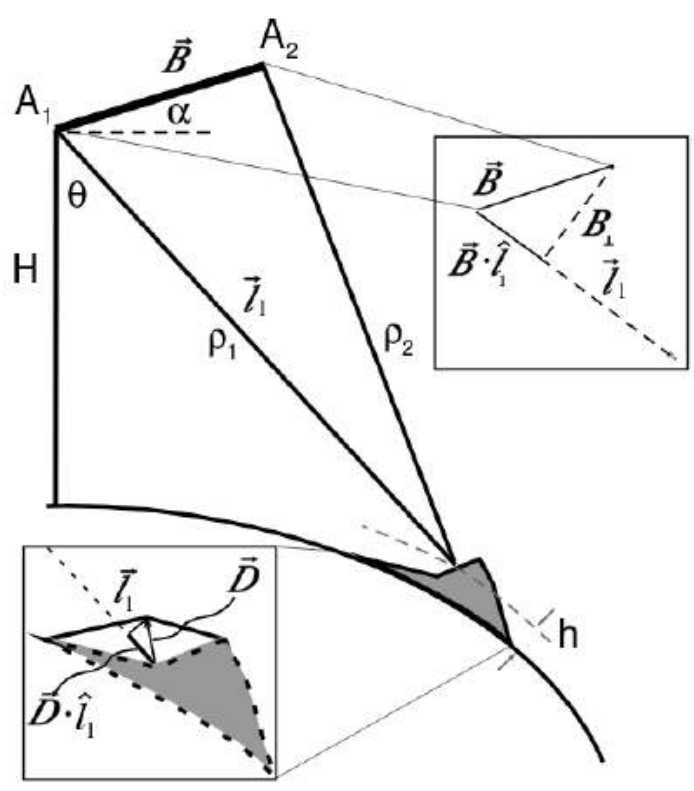

Figure 2.5: Imaging geometry for satellites $A_{1}$ and $A_{2}$, orbiting at an altitude of $H$, with a baseline separation vector $\vec{B}$ of length $B$. The distance from the satellite to the ground for each satellite, $\rho_{1}$ and $\rho_{2}$, is measured along the look vector $\hat{l}_{1}$. The perpendicular baseline $B_{\perp}$ is the dot product of the baseline separation vector and the look vector. The look angle $\theta$ is fixed for each satellite while the angle $\alpha$ varies depending on the baseline separation (Bürgmann et al., 2000a).

The interferometric phase is sensitive to topographic changes when observing the same scene from different observation geometries (i.e. for topographic mapping) or to surface motion when the same scene is observed at different times (i.e. surface deformation caused by an earthquake) (Zebker et al., 1994).

The resulting interferogram is a map of phase change, whereby each fringe or contour represents a phase change of one wavelength ( $2 \pi$ radians) along the twoway travel path (Bürgmann et al., 2000a), representing the amount the ground has moved (i.e. total ground displacement vector $(\vec{D})$ ) in the radar's line-of-sight (LOS) $\left(\vec{l}_{1}\right)$. The LOS displacement of each fringe is equal to half the wavelength $(\pi)$ of the original signal, which for the L-band $(\lambda=23 \mathrm{~cm})$ ALOS1 data, represents $11.5 \mathrm{~cm}$ of LOS displacement.

The interferometric phase $\left(\phi_{I}\right)$ contains contributions from the curvature of the earth $\left(\Delta \phi_{\text {geom }}\right)$, topography $\left(\Delta \phi_{\text {topo }}\right)$, surface deformation $\left(\Delta \phi_{\text {def }}\right)$, orbital error $\left(\Delta \phi_{\text {orb }}\right)$, ionosphere and troposphere delay $\left(\Delta \phi_{\text {atm }}\right)$ and phase noise $\left(\Delta \phi_{\text {noise }}\right)$ (Hooper, 2008):

$$
\phi_{I}=\Delta \phi_{\text {geom }}+\Delta \phi_{\text {topo }}+\Delta \phi_{\text {atm }}+\Delta \phi_{\text {noise }}+\Delta \phi_{\text {orb }}+\Delta \phi_{\text {def }}
$$

The phase contribution from the curvature of the earth $\left(\Delta \phi_{\text {geom }}\right)$ and the phase contribution from orbital errors $\left(\Delta \phi_{\text {orb }}\right)$ are calculated using the baseline distance 
$(B)$, the look angle $(\theta)$, the angle between the reference and the repeat satellites

$(\alpha)$, and the distance between each satellite and the ground $\left(\rho_{1}\right.$ and $\left.\rho_{2}\right)$ [Figure 2.5] (Zebker et al., 1994). The baseline distance $B$ is a measure of the spatial separation between the first and second passes of a satellite when observing the same scene (Bürgmann et al., 2000a). The additional path length $(\delta \rho)$ from orbital errors increases as the baseline distance increases (Zebker et al., 1994):

$$
\delta \rho \approx B \sin (\theta-\alpha)+\frac{B^{2}}{2 \rho}
$$

The phase contribution from orbital errors $\left(\Delta \phi_{\text {orb }}\right)$ is typically removed prior to extracting the phase-difference (Sandwell et al., 2011b). The observation geometry of the two acquisitions is established and a six-parameter affine transformation is completed to warp the repeat image, or 'slave' into alignment with the reference image, or 'master', with sub-pixel accuracy (Bürgmann et al., 2000a). The slave image is aligned to the master image in such a way that the scene is in effect being observed from exactly the same geometry and phase extraction occurs without the contribution from the orbital errors $\left(\Delta \phi_{\text {orb }}\right)$ described above.

The topographic contribution $\left(\Delta \phi_{\text {topo }}\right)$ is removed by subtracting a highresolution Digital Elevation Model (DEM), such as the $30 \mathrm{~m}$ Shuttle Radar Topography Mission (SRTM) DEM, from the total phase value (Massonnet et al., 1993). The quality of the DEM is important, as the uncertainties in the topographic model are propagated and reduce the accuracy of the final phase (Zebker et al., 1994). Transmission delays $\left(\Delta \phi_{a t m}\right)$ due to variations in density of free electrons in the ionosphere and water vapour in troposphere are removed after the formation of the interferogram, as described in Sections 2.4 and 2.4 (Fattahi and Amelung, 2015).

Once the aforementioned contributions have been removed, those produced by surface deformation $\left(\Delta \phi_{\text {def }}\right)$ and residual noise $\left(\Delta \phi_{\text {noise }}\right)$ remain in the final interferogram (Massonnet and Feigl, 1998). The predominant contribution to the noise field is from poor image coherence, with other low-level interference sources such as multi-pathing and channel cross-talk affecting urban areas also generating 
noise (Simons and Rosen, 2015).

\section{Image Coherence}

The correlation value or image coherence $\left(\gamma_{\text {total }}\right)$, is a measure of how similar or different the phase of two SAR scenes are, where 0 is not similar at all and 1 is perfectly matched (Bürgmann et al., 2000a). Coherence is controlled by the spatial $\left(\gamma_{\text {spatial }}\right)$, Doppler $\left(\gamma_{\text {Doppler }}\right)$, thermal $\left(\gamma_{\text {thermal }}\right)$ and temporal $\left(\gamma_{\text {temporal }}\right)$ changes of the image geometry and surface scattering properties (Osmanoğlu et al., 2016):

$$
\gamma_{\text {total }}=\gamma_{\text {spatial }}+\gamma_{\text {Doppler }}+\gamma_{\text {thermal }}+\gamma_{\text {temporal }}
$$

Other factors being equal, interferograms generated from SAR images separated by short intervals of time, short-baseline separations and small changes in look angle will have higher correlation values and a smaller noise component (Hooper, 2008).

Spatial coherence $\left(\gamma_{\text {spatial }}\right)$ is controlled by the baseline distance between the two acquisitions forming an interferogram (Rosen et al., 2000). As interference relies on the random phase component of the two images cancelling, a small phase component relating to the different observation geometries will still be present in the data, unless the images have been aligned with sub-pixel accuracy. The change in path-length between the two acquisitions means the phase will not cancel perfectly and the interferogram will be dominated by 'speckle' without any coherent phase returns (Massonnet and Feigl, 1998). Large-baseline distances are a problem for older satellites such as ALOS1, for which the drift is as much as $6000 \mathrm{~m}$ in a four year period [Figure 3.2]. More recent platforms, such as ALOS2 and Sentinel 1A/B have much better control on orbits and baseline distances vary by approximately $\pm 200 \mathrm{~m}$ over one year [Figure 3.2]. For each system, a critical baseline threshold exists beyond which the phase difference due to the curvature of the earth will exceed $2 \pi$ radians and the resulting interferogram will be completely decorrelated (Sandwell et al., 2011a). For ALOS1 with a look angle of $34^{\circ}$, orbiting at an altitude of $700 \mathrm{~km}$, the critical baseline distance is $6.5 \mathrm{~km}$ for FBD and $13.1 \mathrm{~km}$ for FBS (Sandwell 
et al., 2011a). As a rule of thumb, the optimal baseline distance is one-quarter of the critical baseline distance; longer baseline distances are more susceptible to topographic contributions (Sandwell et al., 2011a).

Temporal decorrelation $\left(\gamma_{\text {temporal }}\right)$ arising from changes in the scattering properties of the ground surface over time is one of the largest limitations to extracting interferometric phase (Wei and Sandwell, 2010). Growth of leafy vegetation or fresh snow cover, among other processes, change the range distance and scattering properties of the ground, producing a small phase shift (Bürgmann et al., 2000a). If the change in LOS distance is less than one phase cycle, the affected pixels may remain coherent. Longer-wavelength (L-band) interferograms perform better than shorter-wavelength systems in highly vegetated regions for the same temporal separations (Wei and Sandwell, 2010). This is due to the longer-wavelength signals maintaining coherence when large changes in LOS distance occur. As an example, L-band (ALOS1) data in a highly vegetated region in California remained coherent with a 2 year temporal separation whereas C-band (ERS-2) interferograms lost coherence after six months (Wei and Sandwell, 2010). Additional contributions to temporal decorrelation can stem from local events such as landslides, erosion, glacier flow and changes in surface water extent (Massonnet and Feigl, 1998).

The Doppler correlation $\left(\gamma_{\text {Doppler }}\right)$ relates to whether the scene is observed at exactly the same look angle and altitude. Decorrelation occurs if the satellite has a sizeable difference in pitch, roll or yaw between acquisitions (Osmanoğlu et al., 2016). The contribution from thermal changes $\left(\gamma_{\text {thermal }}\right)$ to the radar instrument itself is often negligible and typically ignored (Osmanoğlu et al., 2016).

\section{Interferogram networks}

Depending on the intended final use of the interferograms, different pairs of SAR images are selected and networks of interferogram are processed relative to one another. When measuring co-seismic offsets, two-pass processing is used, whereby one interferogram is generated using two SAR images acquired either side of an earthquake (e.g. Hamling et al., 2017, Massonnet et al., 1993, Pritchard et al., 2002). 

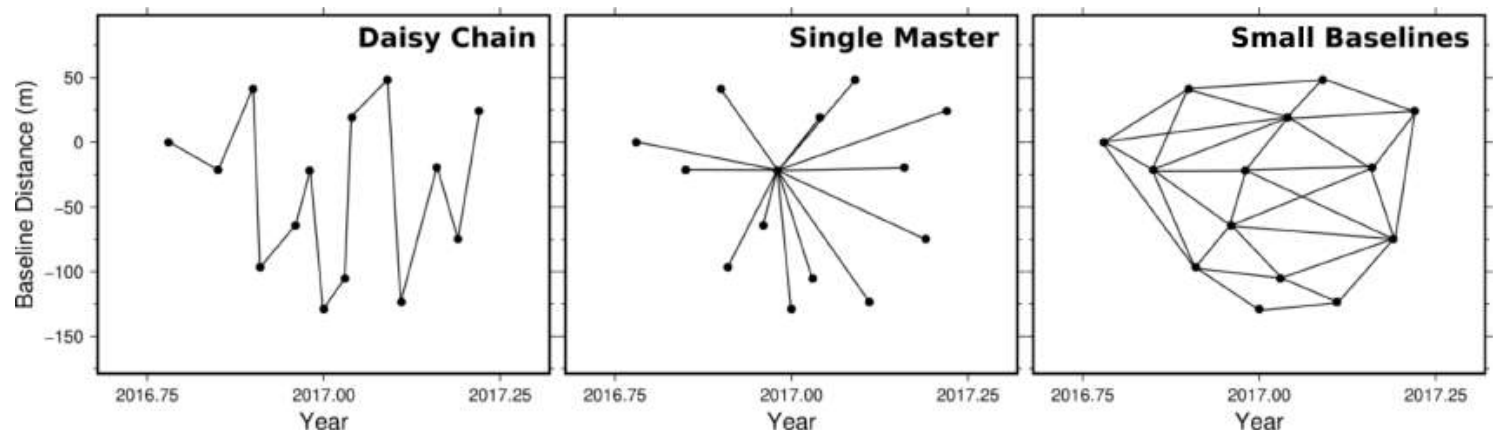

Figure 2.6: Examples of interferogram networks for time series processing. The daisy chain connects acquisitions in chronological order, single master connects all acquisitions to a one image and, small-baselines makes pairs with the temporal and spatial separations below a given threshold.

In contrast, for measuring long-term interseismic deformation, networks of interferograms are constructed so that surface creep can be observed through time (e.g. Bürgmann et al. (2000b), Chaussard et al. (2015), Fialko (2006)).

Figure 2.6 presents different configurations of interferometric pairs in a network. A daisy chain, in which each acquisition is connected chronologically, is useful when baseline distances are short and all acquisitions have high signal-to-noise ratios (SNR), this network is a good first approach for checking for temporally variable deformation (Massonnet and Feigl, 1998). A single master network is used when performing Persistent Scatterers (PS) processing, for example with Stanford Method for Persistent Scatterers (StaMPS) (Hooper, 2008). For time series derived using the Small Baseline Subset (SBAS) method, a network of SAR pairs are selected based on temporal and spatial separation thresholds, yielding many pairs with multiple connected loops within the network (Schmidt and Bürgmann, 2003).

\section{Filtering}

Filtering is used to remove unwanted noise from an interferogram prior to unwrapping. When searching for a regional tectonic signal, short-wavelength noise is removed with a long-wavelength filter, while a short-wavelength filter would be used to identify features such as a localised area of subsidence (Rosen et al., 2000). Two types of filters are typically applied during InSAR processing: a Goldstein-Werner Adaptive filter and Gaussian low-pass filtering (Goldstein and Werner, 1998). 
The power spectrum of an interferogram is mostly characterised by white noise, with only a narrow band related to the phase signal (Goldstein and Werner, 1998). The white noise is mainly due to loss of coherence from topography, baseline geometry and thermal contributions. In the frequency domain, the Adaptive Goldstein-Werner filter has the characteristics of a broadband filter in the noisy regions of the spectrum and a narrow bandpass where the phase signal is present (Goldstein and Werner, 1998). Effectively, the filter adapts to local phase variations: where the phase changes quickly spatially the filter strength is lower and where the phase changes slowly the filter strength increases (Goldstein and Werner, 1998).

A certain level of caution is required when filtering interferograms, as filtering errors can be spatially correlated, and when stacked these can be amplified. The work of Tymofyeyeva and Fialko (2015) suggests that filtering should not be applied when generating a series of interferograms for time series analysis to avoid the introduction of spatially correlated filtering errors to the time-series solution.

\subsection{Unwrapping Interferograms}

To estimate surface displacement, the InSAR image must be 'unwrapped' to produce a relative deformation field from which ambiguous multiples of $2 \pi$ have been removed (Rosen et al., 2000). A 'wrapped' interferogram is an image with fringes of relative phase difference expressed as multiples of the radar's wavelength (Price and Sandwell, 1998). The phase unwrapping process adds $2 \pi$ radians for each full cycle of deformation within the frame, reconstructing the deformation field by shifting each fringe by $2 \pi$ [Figure 2.7] (Massonnet and Feigl, 1998).

The Statistical-Cost, Network-Flow Algorithm for Phase Unwrapping (Snaphu) developed by Chen and Zebker (2000) is one of the most widely applied unwrapping algorithms. The program is largely based on the premise that phase differences around a closed loop of pixels should sum to zero [Figure 2.8] (Chen and Zebker, 2000). The identified residual loops are then used to determine how many full phase cycles must be added to produce the unwrapped phase gradient (Chen and Zebker, 


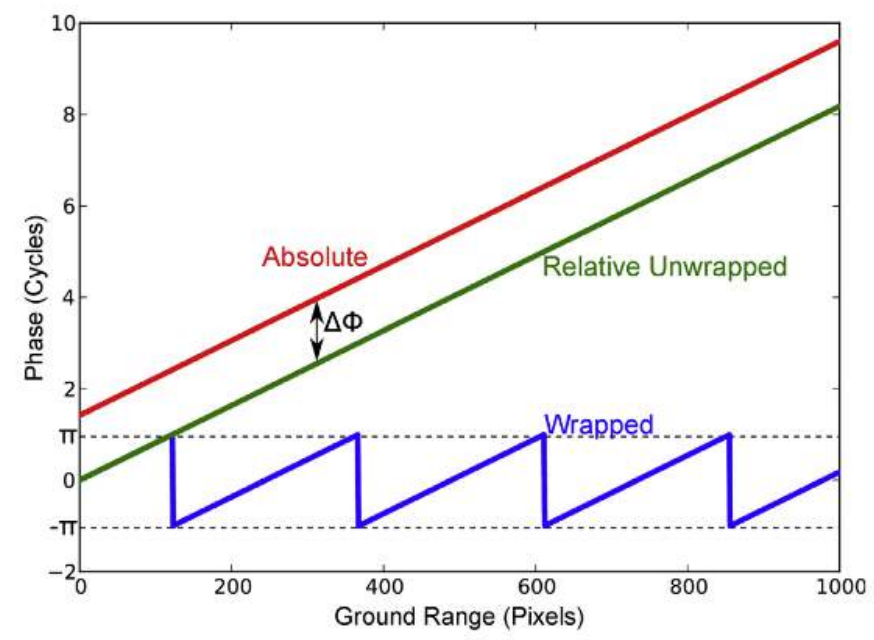

Figure 2.7: A wrapped interferogram with a constant phase change across the scene (from 0 to 1000 pixels) has a cyclic phase measure of $\pm \pi$. Unwrapping the image adds $2 \pi$ for each full cycle, shifting the phase measure to a relative deformation field. GPS data are then used to shift the relative measure of displacement to an absolute reference frame by performing a static shift $(\Delta \Phi)$, aligning the deformation field to the ground measurements. Figure originally from Massonnet and Feigl (1998) adapted by Osmanoğlu et al. (2016).

2000). Snaphu was developed for application to images with uniform geographic sample spacing, which is not always the case (Simons and Rosen, 2015).

Phase aliasing or gaps in data can lead to unwrapping errors where a $2 \pi$ (one phase cycle) step occurs across a boundary with no corresponding ground deformation (Bürgmann et al., 2000a). Phase aliasing results when the true phase changes by more than one cycle between samples. This can arise in regions with steep phase gradients (e.g. near to a fault rupture when the deformation within

$$
\begin{aligned}
0.4-0.1 & =0.3 \\
0.6-0.4 & =0.2 \\
0.9-0.6 & =0.3 \\
0.1-0.9 & =0.2 \\
& =+1.0
\end{aligned}
$$

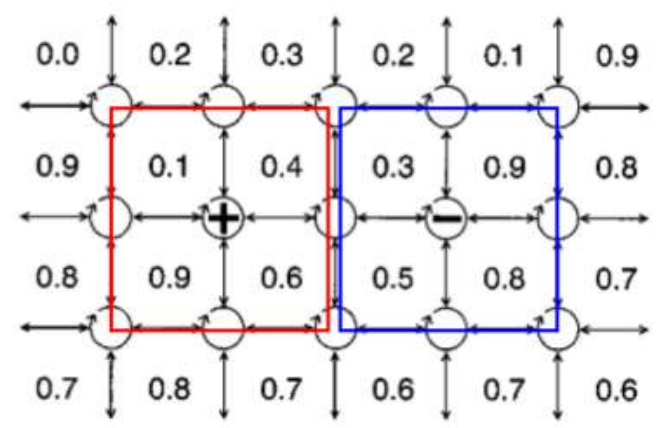

$$
\begin{aligned}
0.9-0.3 & =-0.4 \\
0.8-0.9 & =-0.1 \\
0.5-0.8 & =-0.3 \\
0.3-0.5 & =-0.2 \\
& =-1.0
\end{aligned}
$$

Figure 2.8: Example of closed loop residuals in network equivalent phase unwrapping adapted from Chen and Zebker (2000). Each box represents the wrapped phase measure of one pixel, residuals are calculated around $2 \times 2$ loops. If the sum of the differences is not equal to zero then the loop is identified as a residual. 
Figure 2.9: Schematic of nearest neighbour interpolation for unwrapping from Shanker and Zebker (2009). The circled data are the original data points from a sparsely sampled image. Residuals are calculated in non-overlapping triangular loops and the dense uniformly spaced grid is then inferred by nearest neighbour interpolation.

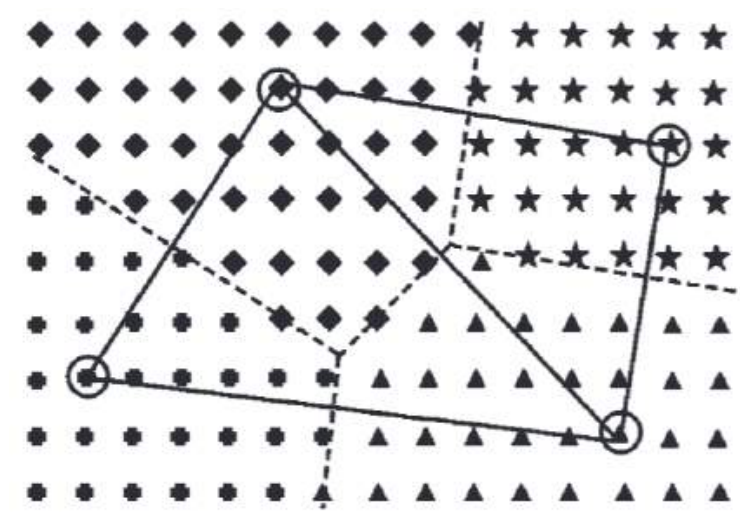

one pixel is greater than $2 \pi$ ), or steep topography, or as a result of large orbital separations (Massonnet and Feigl, 1998). Gaps in data coverage due to water bodies or noise from changes in the scattering properties (i.e. seasonal snow cover or vegetation growth) can under-sample phase changes (Massonnet and Feigl, 1998). The undersampled phase or gaps in data can lead to unwrapping errors in which segments of the unwrapped interferogram have a step in phase of $2 \pi n$, where $n$ is an unknown integer. Unwrapping errors typically occur in parts of the interferogram that are disconnected from the rest of the image (i.e. islands separated by fjords from the mainland) [Figure A6]. These errors can be manually fixed by adding or subtracting the multiples of $2 \pi$ where required.

Shanker and Zebker (2009) developed an interpolation method to improve unwrapping results from sparsely sampled images. This approach uses nearest neighbour interpolation to interpolate missing values in a sparsely sampled region on a regular grid [Figure 2.9]. Snaphu then unwraps the real and interpolated values as usual. One constraint of the interpolation method is that the solution from unwrapping will still be poor if there are not enough input values, regardless of the application of the interpolation before unwrapping (Shanker and Zebker, 2009).

In an interferogram, the noise component arising from ionospheric effects can be manifest as long-wavelength signals spanning the whole frame (Massonnet and Feigl, 1998). Ionosphere delays are produced by variations in the density of free electrons (or total electron content (TEC)) between 50-1000 km above the Earth's surface (Bevis et al., 1992). The highest density of charged particles occurs at an altitude of c. $300 \mathrm{~km}$ (Fattahi and Amelung, 2015). Lower-frequency signals (i.e. 
L-band) experience a greater propagation delay than higher-frequency signals (i.e. C-band). The TEC can be measured directly by comparing the signal from two different frequencies received by the same GPS antenna and the ionosphere contribution then removed from the unwrapped interferogram (Fattahi and Amelung, 2015). A dense network of GPS stations is required for the spatial resolution of TEC measurements to be useful in resolving changes in TEC across an InSAR image (Fattahi and Amelung, 2015). Measurements must also be repeated as TEC changes through time (Fattahi and Amelung, 2015).

Orbital errors from large baseline distances, as described above, also appear as long-wavelength phase ramps. If the perpendicular baseline distance is larger enough in regions with variable topography short-wavelength errors can also occur (Simons and Rosen, 2015). The differences in observation geometry mean the scene is not being viewed from exactly the same perspective and the topographic component of phase is still present in the data set (Massonnet and Feigl, 1998).

Detrending images is a simple and quickly applied method for reducing long-wavelength noise introduced by ionosphere delays and orbital errors (Simons and Rosen, 2015). The long-wavelength contribution is modelled by a linear 'ramp' or biquadratic polynomial, which is then subtracted to remove the trend from the unwrapped interferogram (Fialko, 2006). The ramp is calculated using a far-field portion of the interferogram that is not experiencing deformation (Fialko, 2006). This method works well in regions where deformation is constrained to a small region of an InSAR frame. However, in regions in which deformation is present across the whole scene, a polynomial surface can erroneously remove part of the deformation field and a bilinear surface is preferential (Simons and Rosen, 2015).

InSAR images can be polluted with noise from troposphere delays with spatial variations at a $1 \mathrm{~km}$ scale (Hanssen et al., 1999). Troposphere delays arise from variations in the distribution of water vapour and air pressure (Bevis et al., 1992). In regions of variable topography, the water content can be topographically correlated along valley walls or ridges. This generates topographically correlated noise on much smaller scales than global models of atmospheric water content can 
adequately represent (Hanssen et al., 1999).

As atmosphere (and ionosphere delays) are not correlated with time, stacking images is a way of increasing the signal-to-noise ratio (SNR) (Fialko, 2006). Stacking improves the SNR by averaging out residual noise in the data. Corrections for long-wavelength noise must be completed before stacking or the final stack may be biased as a result (Simons and Rosen, 2015). Stacking interferograms generates a linear deformation rate, this is useful for a fault experiencing constant creep (e.g. Fialko (2006)) at the surface but more detailed time series analysis is required for temporally variable deformation (e.g. Hamling and Hreinsdóttir (2016)).

\subsection{InSAR Time Series}

The detection and monitoring of creeping faults and slow slip regions has been achieved using time series analysis of interferograms (Bürgmann et al., 2000b, Lyons and Sandwell, 2003). A time series of displacement or variable deformation rates can be calculated from a network of interferograms using methods such as PS and SBAS (Berardino et al., 2002, Hooper et al., 2004, Schmidt and Bürgmann, 2003), described below.

The Persistent Scatterers (PS) method is most useful in urban environments where buildings and other structures provided surfaces which enhance pixel coherence through time (Hooper et al., 2004). A PS is a pixel containing an object, such as building, on the ground surface that consistently returns more energy than surrounding surfaces (Hooper et al., 2012). The phase value of the pixel will be dominated by the contribution from the particular PS (Hooper et al., 2004). In rural areas, optimally orientated exposed rocks can act as PS (Simons and Rosen, 2015). The PS method selects pixels which are coherent through time and weights them accordingly. One master image is used and all other images are processed with respect to that image. The accuracy of this method, when deformation is linear through time, can be better than $1 \mathrm{~mm} / \mathrm{yr}$ (Hooper et al., 2012).

The SBAS approach performs a least squares linear inversion to produce 


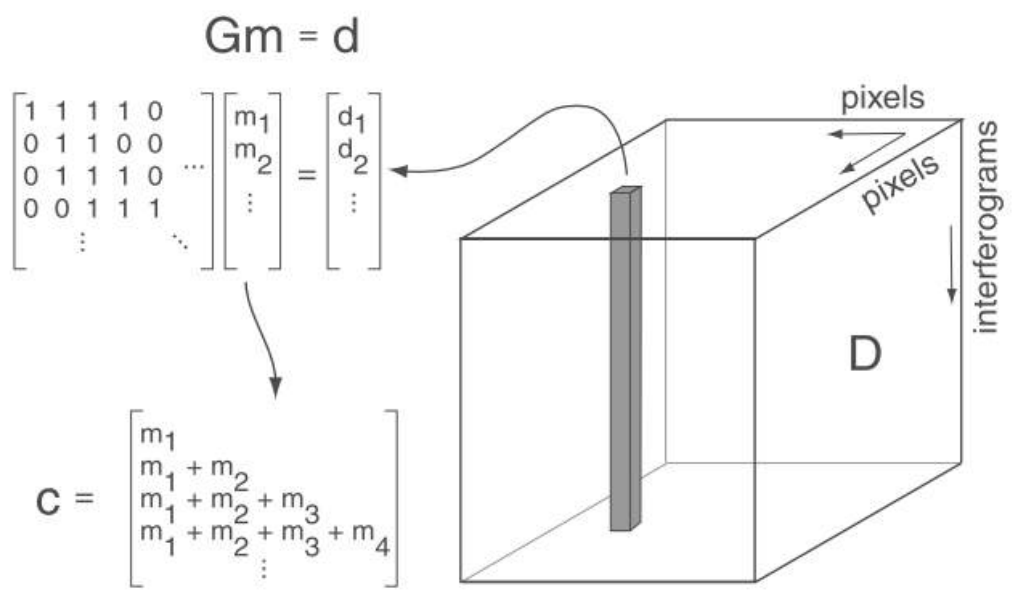

Figure 2.10: Linear inversion problem. Schematic shows relationship between data and model matrices in small baseline method inversion. The inversion is performed pixel by pixel through a stack of interferograms, solving for the pixel displacement at each time step, and applies smoothing factors spatially at completion (Schmidt and Bürgmann, 2003).

a times series of range change deformation (Berardino et al., 2002, Schmidt and Bürgmann, 2003). An inversion is performed on a network of unwrapped interferograms, with short temporal and spatial separations, to solve for deformation at each time step (Hooper et al., 2012). The deformation is solved relative to a stable point within the frame to which each of the interferograms is tied (Berardino et al., 2002). All scenes must be connected to all other scenes through a network of interferograms, if a scene is only linked to one other it will be dropped from the inversion (Chaussard et al., 2015). The inversion is repeated for each pixel that is coherent through all interferograms [Figure 2.10] (Schmidt and Bürgmann, 2003). Ideally, the number of interferograms $N$ is larger than the number of SAR scenes $S$ : when $N$ approaches $S$ the model becomes unstable (Schmidt and Bürgmann, 2003).

The linear SBAS inversion uses a design matrix $G$ which is constructed from the temporal distribution of SAR acquisitions, model parameters $(m)$ and data vector $(d)$ that contains the range change data for each pixel $(i, j)$ in each $\left(k^{t h}\right)$ interferogram (Schmidt and Bürgmann, 2003):

$$
\mathbf{G}_{i j} \mathbf{m}_{i j}=\mathbf{d}_{i}
$$


The error $(e)$, the difference between the modelled $\left(d^{\text {pre }}\right)$ and observed $\left(d^{\text {obs }}\right)$, is calculated at each step:

$$
\left[e_{i}=\mathbf{d}_{i}^{o b s}-\mathbf{d}_{i}^{p r e}\right.
$$

When the error approaches zero the model parameters $\left(m^{e s t}\right)$ predict the observed data (Schmidt and Bürgmann, 2003):

$$
\mathbf{m}^{\text {est }}=\left[\mathbf{G}^{T} \mathbf{G}\right]^{-1} \mathbf{G}^{T} \mathbf{d}
$$

Once obtained, the increments of LOS displacement are summed to produce a time series of cumulative displacement. Each time step uses the same reference pixel, so all deformation is measured relative to a common location (Schmidt and Bürgmann, 2003). The spatial resolution of the time series is determined by the density of coherent pixels through all time steps, while the temporal resolution is dictated by the acquisition rate of the SAR data (Schmidt and Bürgmann, 2003).

Recent adaptations to early SBAS algorithms include coherence selection of interferograms (Chaussard et al., 2015) and closed-loop atmosphere corrections (Tymofyeyeva and Fialko, 2015). Chaussard et al. (2015) developed a coherencebased selection to improve SBAS results with a network of over 3600 interferograms constructed from more than $250 \mathrm{SAR}$ images. Interferograms were included in the inversion if more than $50 \%$ of the pixels within a region of interest had a coherence of more than 0.5. This selection discriminates against interferograms with poor coherence, even if they are within the maximum baseline distance threshold. The atmosphere correction method from Tymofyeyeva and Fialko (2015) is based on the premise that interferograms sharing an acquisition date contain the same contributions from propagation delays. The atmospheric delays are solved for by calculating the residuals of circle tests, where unwrapped interferograms $t_{1}-t_{2}, t_{2}-t_{3}, t_{3}+t_{1}$ should sum to zero and where they don't this difference is attributed to temporally variable atmospheric noise. Once estimated, the atmospheric phase residuals are removed from each time step in the SBAS inversion. 


\subsection{Incorporation of GPS}

The inclusion of GPS in InSAR processing allows for translating the relative deformation field into an absolute reference frame (Bürgmann et al., 2000a). A static shift can be applied so that relative InSAR displacements (e.g. the mean value of pixels within $200 \mathrm{~m}$ of a GPS station) are shifted to the value of local GPS stations, this is often approximated as an additional ramp (Chaussard et al., 2015). This ties the InSAR time series to an absolute value within the frame and can then be used for more detailed analysis of the deformation field.

Integrating GPS has also proven to improve the accuracy of the measured 3D deformation field. Bürgmann et al. (2006) used the horizontal velocity field from a dense GPS network to remove the horizontal component of the InSAR deformation and thereby resolve vertical displacements with sub-mm/yr precision. Fialko (2006) used linear planes calculated between GPS and InSAR images to removed residual contributions from long-wavelength noise to significantly increase the SNR.

A trade-off exists for aligning InSAR with GPS-derived velocities because the two data sets become intrinsically dependent and the GPS cannot be used to estimate errors in the InSAR (Chaussard et al., 2015). By not aligning InSAR to GPS Chaussard et al. (2015) were able to use the GPS network to estimate longand short-wavelength noise within the data, compare final velocities and estimate the uncertainty of the velocity field.

\section{Sub-sample interferograms}

Prior to modelling, InSAR images must be sub-sampled to reduce the number of data points so that modelling can be completed with the computational power available. Uniform (e.g. Pritchard et al., 2002), resolution based, deformation gradient (e.g. Jónsson et al., 2002, Simons et al., 2002) are common methods for achieving this with varying results (Simons and Rosen, 2015). Here we implement an adaptive quad-tree sampling method (gradient-based method) (Bagnardi and Hooper, 2018). 
The quad-tree sample divides the image into quadrants, calculates a low-order bestfit surface and removes it (Bagnardi and Hooper, 2018). If the residual value for the quadrant is greater than a given threshold then the quadrant will be divided again (Bagnardi and Hooper, 2018). This process is continued until the variance in each quadrant is below the specified threshold. This gradient based, quad-tree produced sub-sampled images with sample density higher closer to the deformation source and requires no prior knowledge of the fault geometry (Bagnardi and Hooper, 2018).

\subsection{Modelling}

Modelling of co-seismic and post-seismic deformation is completed with a simple source model implemented with a statistical Bayesian inversion approach. Deformation is modelled as a rectangular dipping fault with uniform slip embedded in an isotropic elastic half-space with an assumed Poissons's ratio $v=0.25$ (Bagnardi and Hooper, 2018, Okada, 1985). The fault length, width, depth, dip, strike, location, amount of stike-slip and dip-slip are determined. Bayesian modelling generates posterior probability density functions (PDFs) which determine the likelihood of a range of source parameters given the deformation pattern and model bounds specified (Bagnardi and Hooper, 2018). Unlike direct-search inversion methods, the Bayesian approach is able to appropriately characterise uncertainties associated with estimates of source parameters by presenting credible intervals for each value (Bagnardi and Hooper, 2018). Posterior PDF are generated for each source parameter and an optimal set of source parameters is identified using the maximums of each distribution (Bagnardi and Hooper, 2018).

The data vector $d$ is equal to the product of the non-linear function $G$ and model parameters $m$, and the error $\epsilon$ :

$$
d=G(m)+\epsilon
$$

The probability of a set of model parameters given how well those parame- 
ters fit the data, with a certain uncertainty, is described by a conditional posterior PDF $p(m \mid d)$ (Bagnardi and Hooper, 2018):

$$
p(m \mid d)=\frac{p(d \mid m) p(m)}{p(d)}
$$

This posterior PDF is calculated for a single set of data with:

$$
p(d \mid m)=(2 \pi)^{-N / 2}\left|\sum_{d}\right|^{-\frac{1}{2}} \times \exp \left[-\frac{1}{2}(d-G m)^{T} \sum_{d}^{-1}(d-G m)\right]
$$

where $N$ is the total number of data points and $\sum_{d}^{-1}$ is the inverse of the variancecovariance matrix for a given data set (Bagnardi and Hooper, 2018). The upper and lower bounds of a outcome are set, where within these bounds each value is equally probable and outside the probability is set to zero, $p(m)=0$.

\subsection{Determining Uncertainty in Interferograms}

Outlined below are methods for identifying and quantifying error which were applied throughout processing. These were used to validate processing choices and quantify uncertainty in the result. Estimation of spatial error in interferograms is important as errors propagated through to time series analysis and modelling, may affect the final interpretation. Examination of error and uncertainty must be undertaken to assess the accuracy of any processing corrections applied, to understand the limits of the data, and to provide a level of uncertainty for results produced.

\section{Standard Deviation}

A simple and meaningful measure of noise is the standard deviation of the phase (Goldstein and Werner, 1998). This is a useful method for determining whether a correction such as filtering has reduced noise by comparing the standard deviation before and after the correction (Goldstein and Werner, 1998). 


\section{Circular Tests}

Circular tests can be completed to find unwrapping errors and any processing artefacts which have been added to the data in a connecting loop. If three interferograms are summed, the result theoretically should be zero: a non-zero result indicates the presence of a processing error or noise within the image (Massonnet and Feigl, 1998). Tymofyeyeva and Fialko (2015) presented results from circular testing that showed that filtering interferograms added spatially correlated error. When stacked, this spatially correlated error could be mistaken for a deformation signal. Similarly, when unwrapping interferograms a circular test can be applied to search for unwrapping errors (Shanker and Zebker, 2009).

\section{Comparison with Independent Data}

When both the ascending and descending velocity maps are available, the horizontal InSAR velocities can be compared with GPS velocities to calculate the average absolute deviation between the two data sets (Chaussard et al., 2015). Figure 2.11 presents the results obtained by Chaussard et al. (2015). Fault-parallel velocities were compared, a best fit linear regression was calculated and InSAR velocity field uncertainty was calculated as the average absolute deviation from the regression slope (Chaussard et al., 2015). In the case where the InSAR and GPS are not well correlated, this may indicate that the InSAR does not represent the ground deformation field and careful consideration of processing steps needs to be completed.

\section{Semivariogram}

A semivariogram estimates spatial variation by calculating how values differ with increasing separation (Bagnardi and Hooper, 2018). Variance typically increases with distance, such that two data points close to each other are likely to be similar, and two points further away are more dissimilar [Figure 2.12]. Bagnardi and Hooper (2018) used a semivariogram to estimate noise levels in interferograms prior to modelling. The variance of the interferogram is described by a nugget, range and 

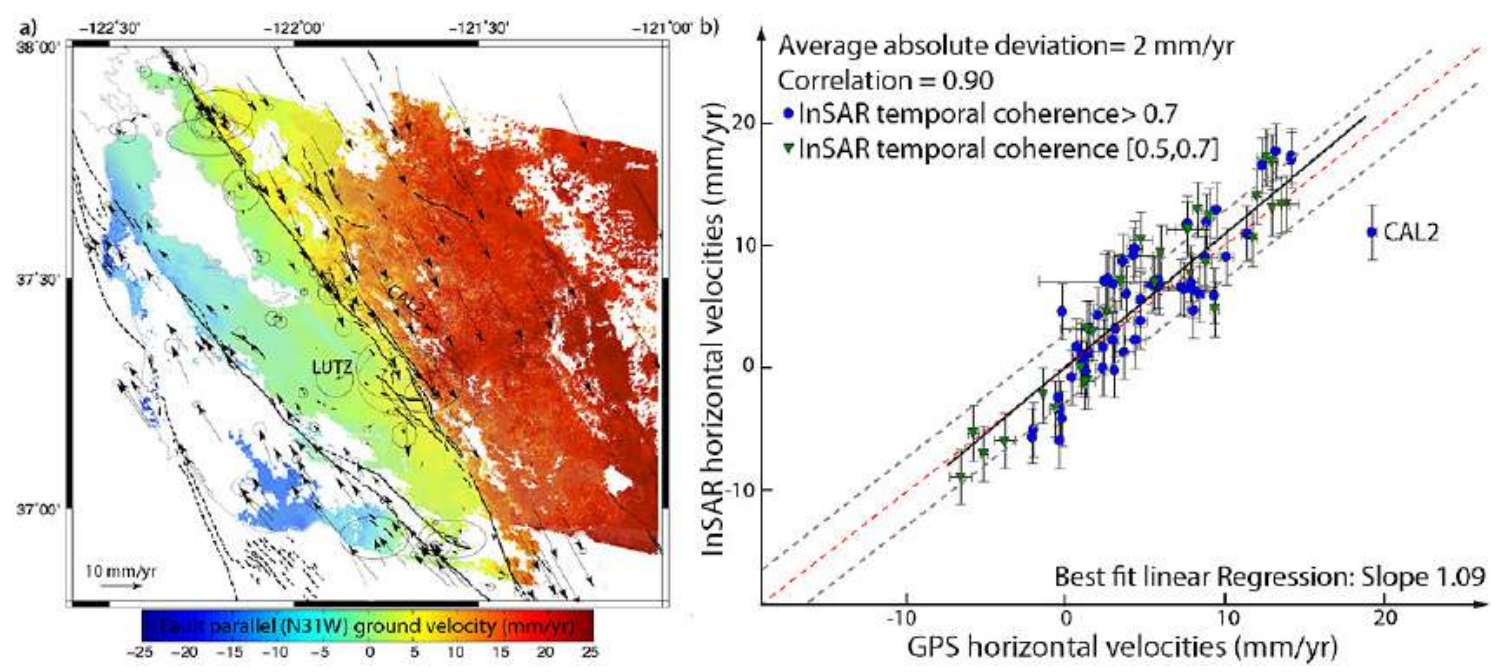

Figure 2.11: Comparison of GPS and InSAR derived velocities by Chaussard et al. (2015). The uncertainty for the InSAR velocity field was determined by finding the average absolute deviation (grey dashed line) from the best fit linear Regression (black line). The InSAR velocities shown are the mean values from within $200 \mathrm{~m}$ of the corresponding GPS location.

sill value (Bagnardi and Hooper, 2018). The nugget is the lowest level of variance in the data measured between two neighbouring locations. The nugget value represents measurement errors or spatial sources of variation at distances smaller than the sampling interval (Bagnardi and Hooper, 2018). The range is the lag distance between the nugget variance and the point at which the variance plateaus. The sill variance is the value at which the variance plateaus indicating that variance no longer increases with distance above that threshold (Bagnardi and Hooper, 2018). Bagnardi and Hooper (2018) suggest that a semivariogram is calculated over a non-deforming region at least as large as the deforming region being investigated. 

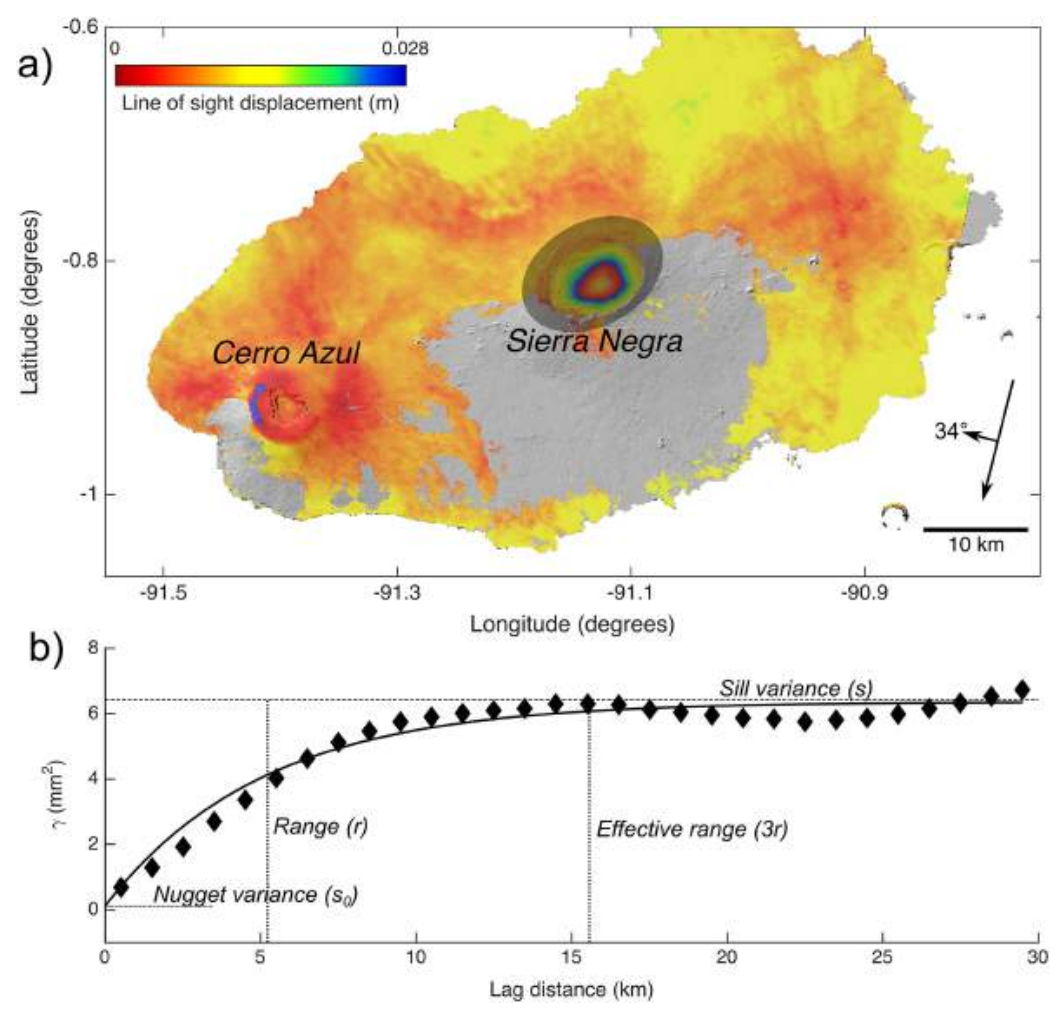

Figure 2.12: Estimation of InSAR noise field of interferogram from southern Isabela Island, Galápagos (Ecuador) by Bagnardi and Hooper (2018). Within the unwrapped interferogram, the deformation is masked (grey shaded region (a)) and the nugget, range and sill variance is estimated from the non-deforming regions. (b) shows how the variances changes with increasing distance (lag) between data values for the experimental (diamonds) and theoretical (solid line) semivariograms. 


\section{Methodology}

In the previous chapter, we outlined the theoretical premise for Interferometric Synthetic Aperture Radar (InSAR). In this chapter, we describe GMTSAR InSAR processing, Small Baseline Subset (SBAS) time series analysis, and Geodetic Bayesian Inversion Software V1.0 (GBIS) modelling. We indicate adaptations to the standard processing that improve results and present the preliminary findings which informed these adaptations. Examples from ALOS2 and Sentinel 1A/B presented in this chapter highlight challenges within the ALOS1 dataset (Section 3.2) and indicate the limitations of these datasets (Section 3.3). All parameter testing was completed on track 348, frame 6260 (ALOS1), as this region is the search area for surface deformation signals near Secretary Island that may indicate the presence of triggered slip.

\subsection{Data}

We analyse Synthetic Aperture Radar (SAR) data from Japan Aerospace Exploration Agency (JAXA) (Advanced Land Observing Satellite's 1 and 2 (ALOS1/ALOS2)) and European Space Agency (ESA) (Sentinel 1A/B) and Global Positioning System (GPS) data from Beavan et al. (2010) [Table 3.1]. The ALOS1 dataset includes 21 ascending acquisitions along track 348, and 12 along track 349 made between December 2006 to January 2011. In addition, there were 2 descending acquisitions along track 639 made between July 2008 and July 2009 [Figure 3.1]. The ALOS2 dataset includes 12 ascending acquisitions made between November 2014 to November 2017. The Sentinel dataset includes 17 ascending acquisitions made between November 2016 and April 2017, and 11 descending acquisitions between October 
2016 to March 2017. The ALOS1 data have an average time interval of 73.5 days; most acquisitions occurred every 46 days although a few have separations of up to 138 days [Figure 3.2]. Here we processed both L-band (ALOS1/ALOS2) and C-band (Sentinel 1A/B) to compare and contrast the results.

\begin{tabular}{llllllll}
\hline Satellite & Agency & Orbit & Frame & Pass Direction & Band & Time Period & No. acquisitions \\
\hline ALOS1 & JAXA & 348 & $6240,6250,6260$ & Ascending & L & $2006-2011$ & 21 \\
ALOS1 & JAXA & 349 & 6240,6250 & Ascending & L & $2006-2011$ & 13 \\
ALOS1 & JAXA & 639 & $4530,4540,4550$ & Descending & L & 2009 & 2 \\
ALOS2 & JAXA & 106 & 6260,6270 & Ascending & L & $2014-2017$ & 12 \\
Sentinel 1A/B & ESA & 96 & & Ascending & C & $2016-2017$ & 17 \\
Sentinel 1A/B & ESA & 44 & & Descending & C & $2016-2017$ & 11 \\
\hline
\end{tabular}

Table 3.1: Summary of data sources: Advanced Land Observing Satellite's 1 and 2 (ALOS1/ALOS2) from JAXA, Sentinel 1A/B from ESA. Ascending data is collected as the satellite orbits from south to north, and descending from north to south.

\subsection{Anticipated Challenges}

The Fiordland region is characterised by steep terrain, vast regions of heavy vegetation, heavy rainfall and seasonal snow cover, each of which contributes to reducing image coherence. The ALOS1 dataset was additionally limited by large orbital drift (up to $6 \mathrm{~km}$ in 2.5 years) and long temporal separations between acquisitions (up to 138 days).

\section{Topography}

Areas where the topographic gradient exceeds the satellites look angle result in reduced spatial coverage of the SAR image. 'Shadows' form where the wavefront is reflected off one side of an elevated feature and does not illuminate the other [Figure 2.2] (Massonnet and Feigl, 1998). Topography also affects the amplitude of the reflected signal: a slope perpendicular to the look direction will reflect more signal than a parallel slope (Massonnet and Feigl, 1998). As the data used here was acquired on right-looking platforms and along ascending tracks, the valley walls dipping towards the southwest are optimally orientated for this orbit geometry, while steep valley walls dipping towards the northeast may be in the shadow and have 


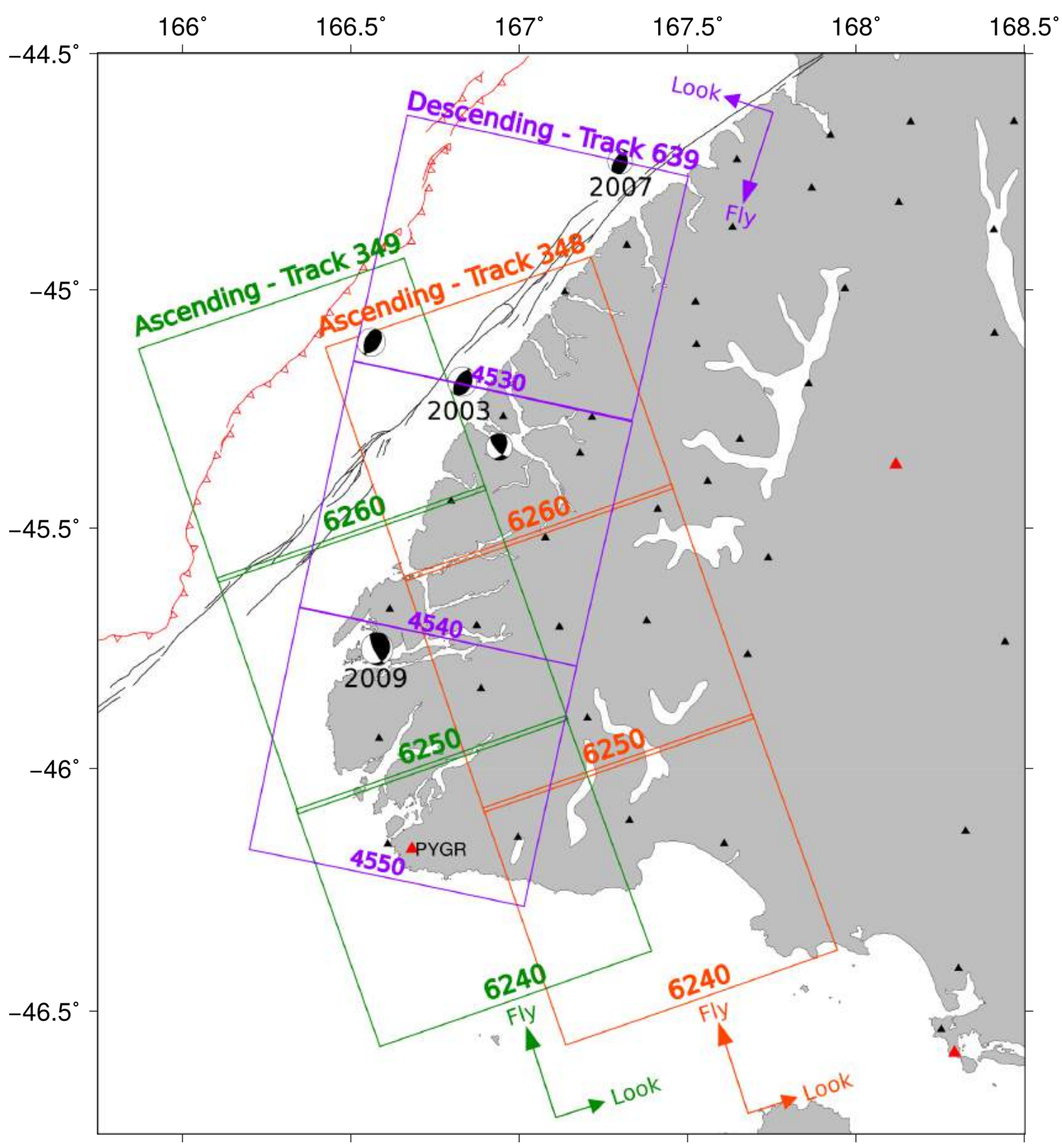

Figure 3.1: ALOS1 SAR frames used in this study. Ascending track 349 and descending track 639 is used to re-analysis deformation from Dusky Sound earthquake. Track 348, frame 6260 is centred on Secretary Island and is used to isolate potential areas of reactivated slip around Secretary Island. Red triangles are cGPS stations, of which PYGR is the only station within the frames. Black triangles are sGPS sites which are occupied as part of the national network approximately every eight years and following large earthquakes (Beavan et al., 2016). Focal mechanism show locations (from southeast to northwest) of the 1989, 2003 and 1993 earthquakes near Secretary Island, as well as the 2007 George Sound (north), and the 2009 Dusky Sound (south) earthquakes. 


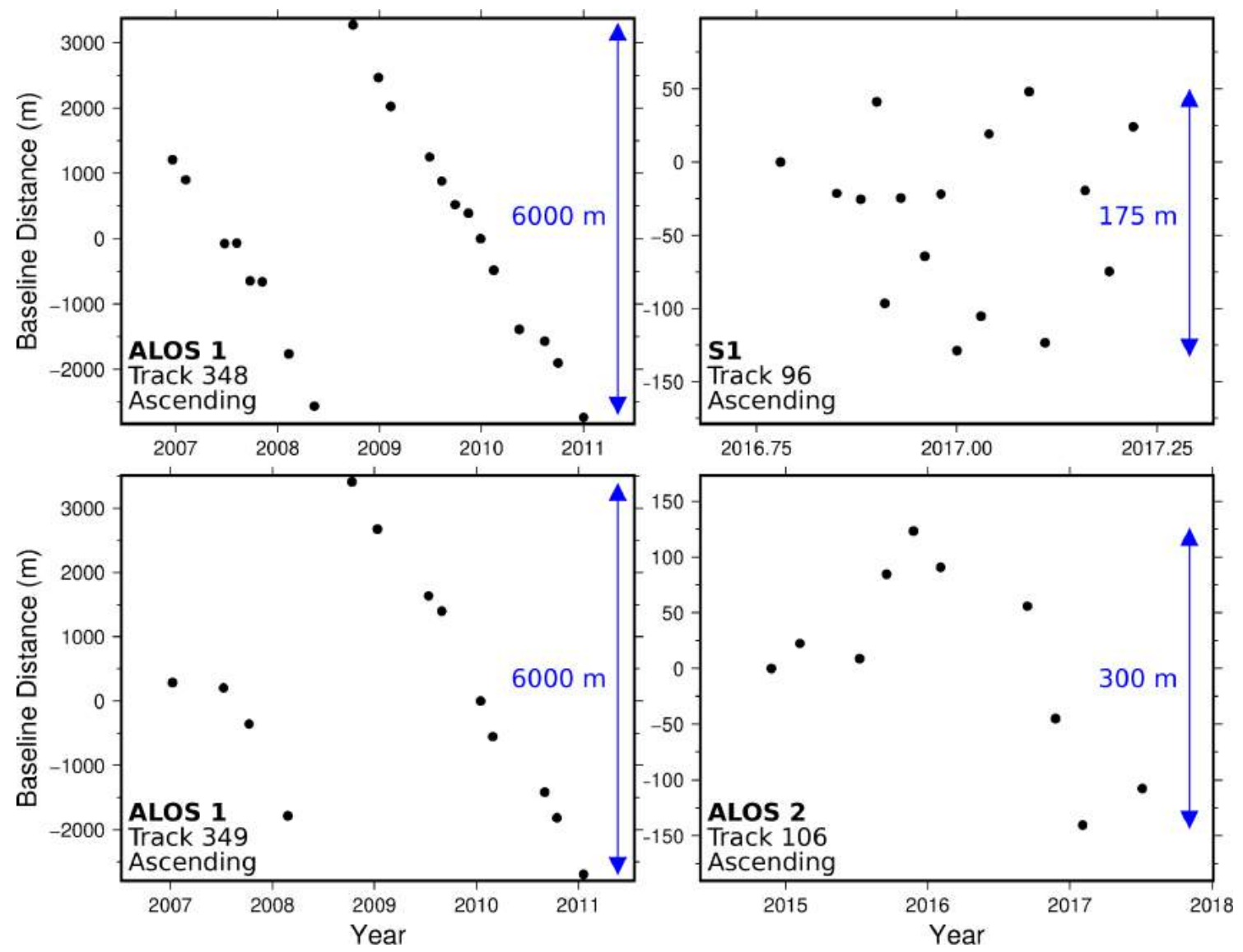

Figure 3.2: Plots of perpendicular baseline separation and temporal separation of SAR acquisitions. The orbital drift of ALOS1 results in baseline distances of $6 \mathrm{~km}$ over 2.5 years. The newer satellites, Sentinel 1 (S1) and ALOS2, have greater orbital control and result in baseline distances of less than $300 \mathrm{~m}$. 
limited spatial coverage.

\section{Heavy Vegetation}

Fiordland contains New Zealand's largest national park with land cover dominated by native forest, tussock and snow-capped mountains. As described in Sections 2.2 \& 2.4, long-wavelength ALOS1/ALOS2 L-band has the ability to penetrate through some vegetation and maintain coherence when small (centimetre scale) changes occur, whereas the coherence of short-wavelength Sentinel 1A/B C-band decreases rapidly with time (Bürgmann et al., 2000a).

\section{High Precipitation}

Fiordland has the highest median annual total rainfall in New Zealand, typically receiving 1500-6000 mm/yr (NIWA, 2012). Precipitation increases with elevation and reduces east of the main divide [Figure 3.3]. As described in Section 2.4, heavy rainfall causes topographically correlated increases in water vapour content which delay the return of the signal to the radar.

\section{Observing Geometry}

Figure 3.2 shows the baseline and temporal separation of the data analysed here. The ALOS2 and Sentinel datasets have a maximum baseline separation of less than $300 \mathrm{~m}$, whereas the ALOS1 platform has an orbital drift of up to $6 \mathrm{~km}$ over the 2.5 years of acquisitions. Orbital drift leads to deviations in the observational geometry of the SAR scene which increase baseline distance and decrease image coherence (see Section 2.3)(Simons and Rosen, 2015). The temporal resolution is also limited by the long-baseline distances (see Section 3.3). 


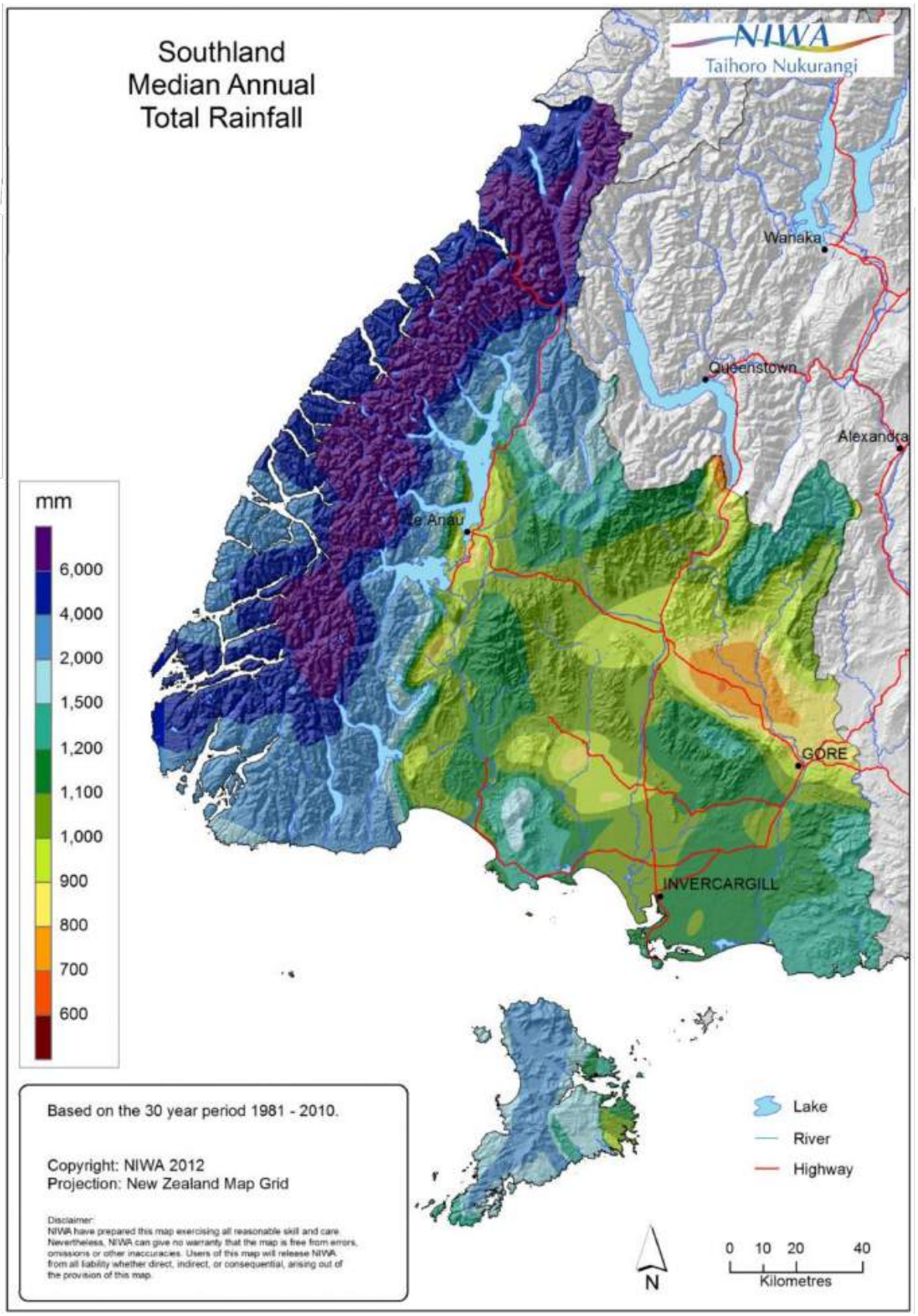

Figure 3.3: Median annual total rainfall for Southland, New Zealand. Note the high levels of precipitation along the South Island's west coast and at higher elevations (NIWA, 2012). These are regions where local atmospheric delays may be present in the data. 


\subsection{InSAR Processing}

We used GMTSAR, an open-source program which calls on five main C algorithms and the standard Generic Mapping Tools (GMT) package with c-shell wrappers (Sandwell et al., 2011b, Wessel and Smith, 2013). GMTSAR calls on the StatisticalCost, Network-Flow Algorithm for Phase Unwrapping (Snaphu) as described in Section 2.4 and Figure 3.4 (Chen and Zebker, 2000). GMTSAR supports two-pass processing, which takes two SAR frames to generate one interferogram, and batch processing, using a series of SAR images to generate many interferograms as input for time series analysis. Batch processing differs from the processing flow described in Figure 3.4, in that: 1) all images are pre-processed with the same orbital geometry (zero Doppler, Earth radius and near-range values) and; 2) all slave images are aligned to the same master.

Interferograms of co- and post-seismic deformation of the Dusky Sound earthquake were processed using the two-pass method [Figure 3.4]. Data from ALOS1 track 348, frame 6260, were processed using batch processing, the additional steps are described throughout the following sections. At the time of processing, no GMTSR batch processing scripts for ALOS2 data were available. Initial processing, as part of the preliminary investigation with ALOS2, was completed using the twopass processing method [Figure 3.4]. These results did not indicate the presence of localised deformation so no further attempts to complete batch processing were made [Figures 4.2 and A5].

The descriptions below of GMTSAR-specific processing steps are paraphrased from the GMTSAR manual Sandwell et al. (2011a), personal communications with David Sandwell at the 2018 UNAVCO InSAR Processing and Theory with GMTSAR short course, and from comments within the GMTSAR scripts. 


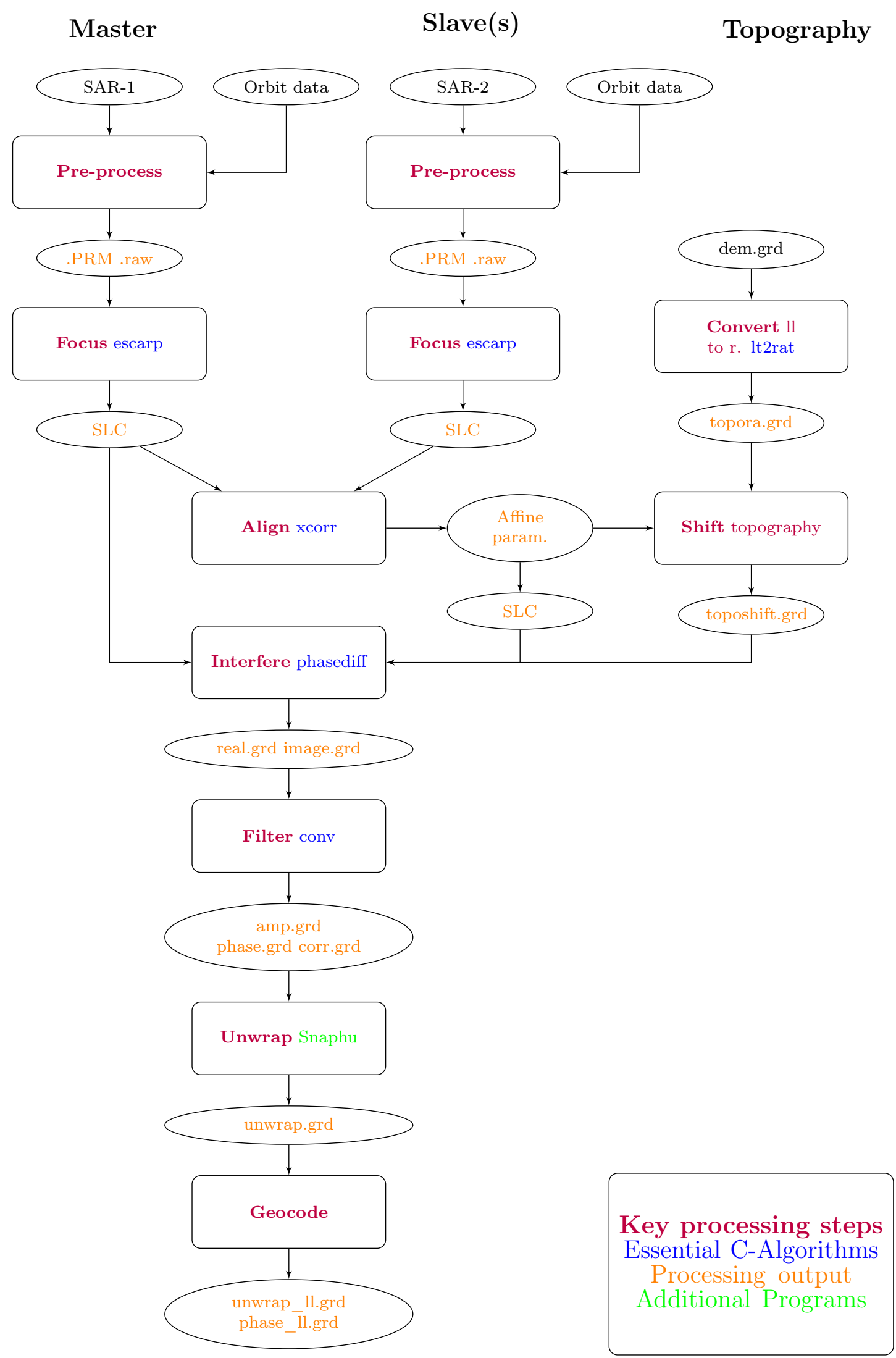

Figure 3.4: Typical processing flow for generating interferograms with GMTSAR. For two-pass processing, only one slave image is used while for batch processing many slave images are all aligned to the same master image. Once aligned, any combination of SLC's can be used to generate interferograms. 

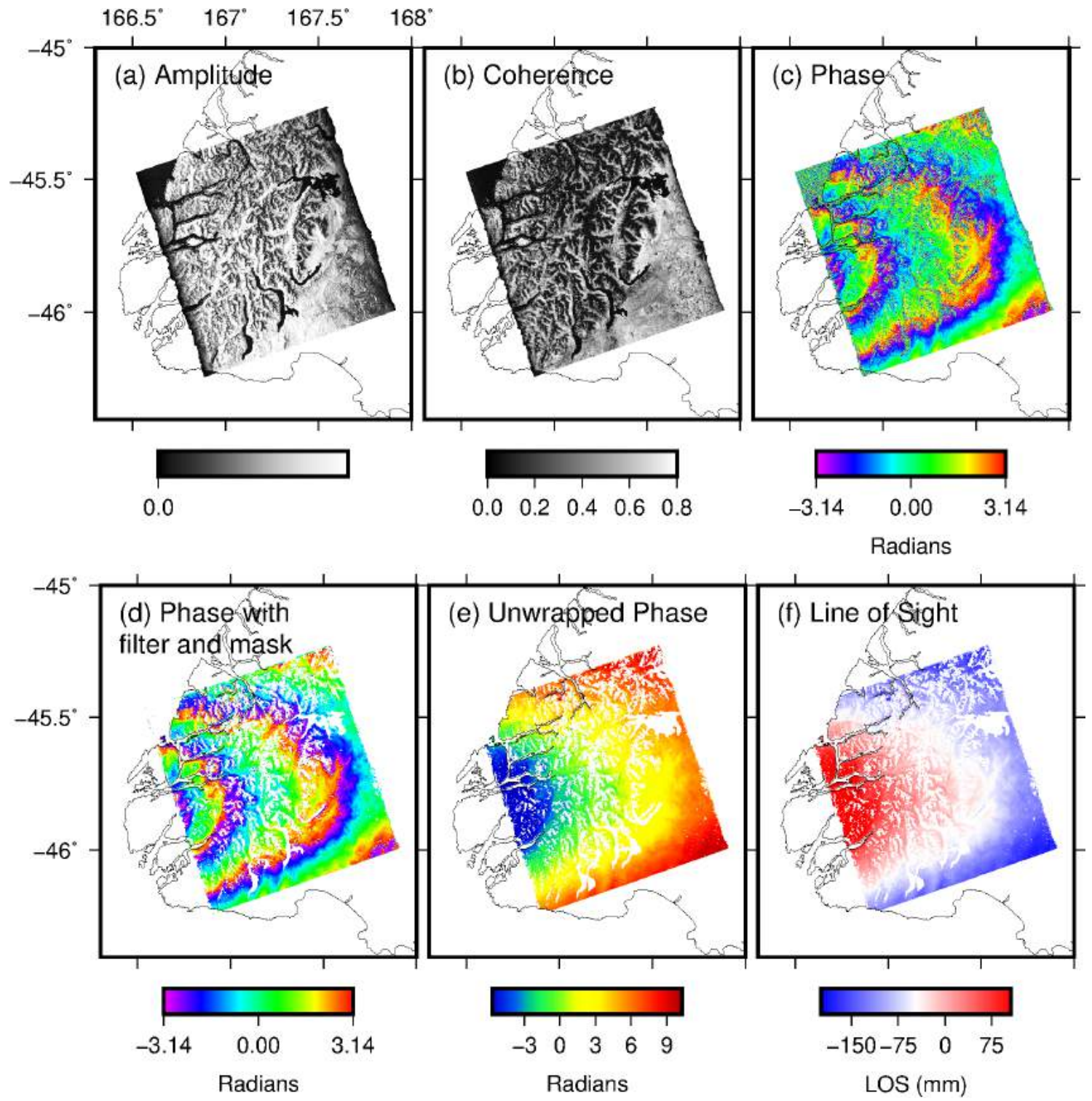

Figure 3.5: Representative output from GMTSAR showing displacements for Dusky Sound earthquake between 28 June 2009 to 13 August 2009 (track 348, frame 6250). (a) Amplitude of reflected back scatter energy. (b) Image coherence. (c) Wrapped phase in radians, each fringe represents c. $11 \mathrm{~cm}$ of deformation. (d) Wrapped phase with $200 \mathrm{~m}$ Gaussian filter and coherence masked applied. (e) Unwrapped phase in radians. (f) Line-of-sight (LOS) displacement in millimetres. 


\section{Pre-process and SLC Formation}

The raw SAR images were pre-processed to convert data from its native format to a generic format for single look complex (SLC) formation. GMTSAR extracted the orbit information from the header of the ALOS1 and ALOS2 raw data or from the auxiliary files for Sentinel-1. The master was selected from the perpendicular baseline plots to minimise baseline and temporal separations (Figure 3.2). The Earth radius, near-range distance and the zero-Doppler distance specific to the master file were defined in the configuration file and all repeat images are focused using those same parameters. The SAR focusing algorithm in GMTSAR is escarp, which focuses thousands of consecutive echos into a SLC format through the method described in Section 2.2 (Sandwell et al., 2011a).

\section{Alignment}

Correct alignment of images removes the geometric contribution to the phase due to differences in the viewing geometry of the scene (see Section 2.3). GMTSAR uses precise orbits to estimate the azimuth and range shifts required to align the upper left corner of the slave image to the master (to within 2 pixels in range and 1 pixel in azimuth) (Sandwell et al., 2011a). GMTSAR computes a two-dimensional cross correlation to calculate values required to warp the slave image into alignment with the master image. GMTSAR defines a template size of $128 \times 256$ pixels and sets the image sub-patch size to double that of the template. The template of the slave image is cross correlated with a sub-patch of the master SAR image. The cross-correlation (CC) was repeated at 20 locations in the range direction and 50 locations in the azimuth direction, totalling to 1000 CCs. The GMTSAR alignment output contains the required shift to align the slave to the master in range and azimuth for each CC location and the signal-to-noise ratio (SNR) for each CC. These range and azimuth shift values were used to calculate the 6 parameters required to shift, stretch or rotate the slave image in range and azimuth to align it with the master image. The SLC for the slave image was then reprocessed using these 6 parameters with an affine 
transformation that preserves points, straight lines and planes within the image.

The CC SNR was examined to estimate the coherence of the final image. Ideally, the SNR for each CC should exceed 20 for a robust solution. Appendix Table 1 summarises tests undertaken to determine the ideal CC parameters for the data in this study. A longer window length results in a higher SNR providing a more robust solution, however, this also increased computation time. Increasing the number of CC decreases the SNR while increasing computation. The change in computation time is more sensitive to increasing the number of CC (nx, ny) and the average SNR is more sensitive to changes in the template size (x_search, $\mathrm{y} \_$search).

When completing GMTSAR batch processing, each of the slave images must be aligned to a 'primary master' so that all interferograms can be generated relative to the same acquisition geometry. Figure 3.6 shows the alignment of SAR images from ALOS1. The primary master for processing was acquired on 29 December 2009. This SAR image was selected as the master as it was closest to the mean baseline distance, acquired in the summer when snow cover was minimal, and acquired with Fine Beam Dual Polarization (FBD) (see Section 2.2) so could be down-sampled to Fine Beam Single Polarization (FBS) format. As the baseline distances are very large for ALOS1, 'secondary masters' were selected in order to align the most distant acquisitions. First, alignment of the primary master to each of the secondary masters and to images within c. $\pm 1300 \mathrm{~m}$ of the primary master was completed. Next, all remaining images were aligned to the secondary masters. As the SLCs of the secondary masters are reprocessed and warped to align with the primary master before subsequent images are aligned to them, all images are, in effect, aligned to the signal primary master. As the baseline distances were small (less than $200 \mathrm{~m}$ ) for Sentinel 1A/B, all slaves images were aligned to a single master image [Figure A1].

To determine whether the final result was biased by the master selection, all data from track 348, frame 6260, were reprocessed with a different master acquired on 8 November 2007, which also fulfilled the criteria listed above [Figure A2]. The noise levels and average coherence values of the interferograms were comparable for 


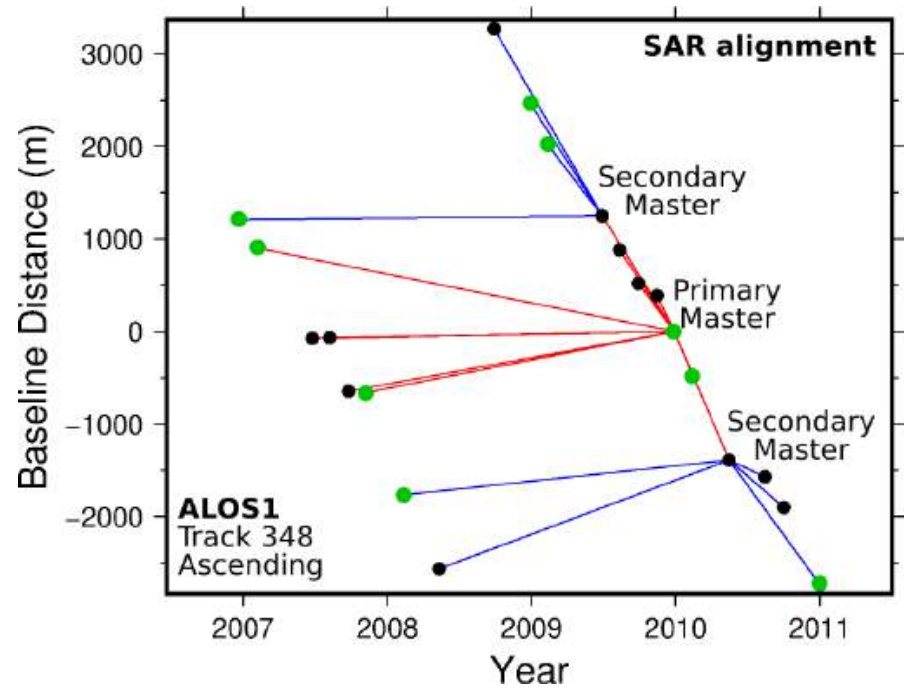

Figure 3.6: Baseline distance vs. time plot showing how each SAR image is aligned to either a 'primary' or 'secondary' master. SAR images within a baseline distance of c. $\pm 1300 \mathrm{~m}$ from the 29 December 2009 acquisition are aligned to this 'master' image. All other SAR images are aligned to the secondary masters acquired on 28 June 2009 and 16 May 2010. As the secondary masters are aligned to the primary master before other pairs all SAR images are effectively aligned to the primary master.

both masters. From here on, ALOS1 results and methods presented will be aligned to the primary master acquired on 29 December 2009, as in Figure 3.6.

\section{Weighting for Persistent Scatterers}

GMTSAR includes tools to weigh Persistent Scatterers (PS) pixels in a SAR image prior to interferogram formation. Each pixel in the focused SAR image (SLC) is assigned a weight based on its amplitude dispersion through time. Figure 3.7 shows the weighting of PS pixels for Fiordland compared with a region in Jakarta, Indonesia. In the example from the mixed land use region surrounding Jakarta, urban areas show as bright regions where the pixels have been up-weighted. The rural areas around the outside of the frame have been down-weighted, as the returned amplitudes varied through time. The pixel weightings for Fiordland show few 'bright spots' as there are few man-made or natural structures in the region that act as PSs. Some pixels are up-weighted: these are randomly dispersed across the image and may represent optimally oriented rock exposures. As compared with ALOS1, the Sentinel 1A/B data have a high proportion of up-weighted pixels. 
Fiordland: ALOS1

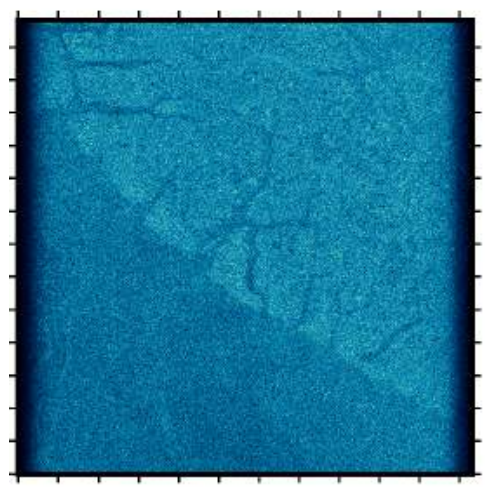

Fiordland: Sentinel 1A/B
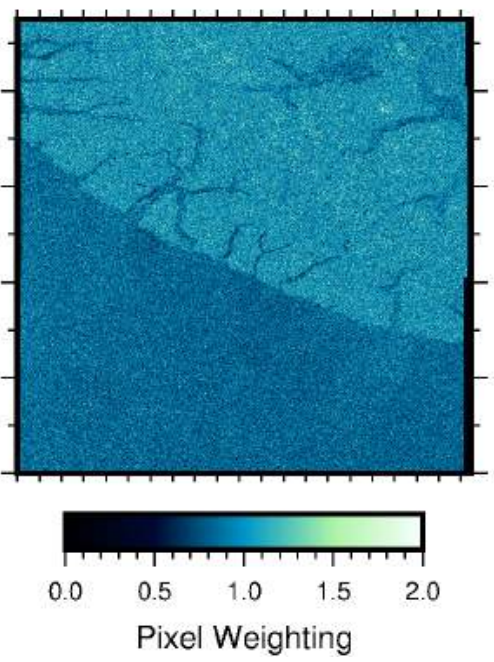

Jakarta: Sentinel 1A/B

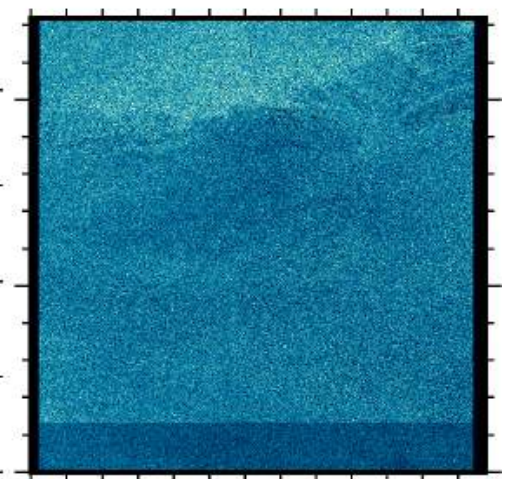

Figure 3.7: Comparison of Persistent Scatterers from Fiordland (rural) and Jakarta (urban/rural). Map of pixel weighting based on amplitude dispersions for Fiordland (ALOS1 (left), Sentinel 1A/B (middle)) and Jakarta (Sentinel 1A/B (right)). Unpublished data for Jakarta from Andy McNab (pers. comms., 2019).

\section{Generating Interferograms}

GMTSAR computes the phase difference between two SAR images, removes the effects of the curvature of the earth and removes the topographic contribution to the total phase (Sandwell et al., 2011a). The general methods outlined in Section 2.3 are used. A $30 \mathrm{~m}$ Digital Elevation Model (DEM) generated as part of the Shuttle Radar Topography Mission (SRTM) mission in 2000 was used to subtract the topographic component of phase from the interferograms.

The effect of summer/winter pairing and spatial/temporal separations on image coherence were tested to determine which pairs of images to connect for the optimal network configuration. Figure 3.8 shows coherence maps of a selection of summer-to-summer pairs, acquired when snow cover is less and coherence between summer-to-summer pairs should in principle be higher (summer SAR acquisitions highlighted in Figure 3.9a). Due to the baseline drift of ALOS1, summer-to-summer pairs are restricted to having either short temporal separations within the same summer, or temporal separations exceeding two years. Coherence was high for pairs with short temporal and baseline separations (Figure 3.8 top-left and bottom-right), and poor for pairs with long temporal and short-baseline separation. Acquisitions 


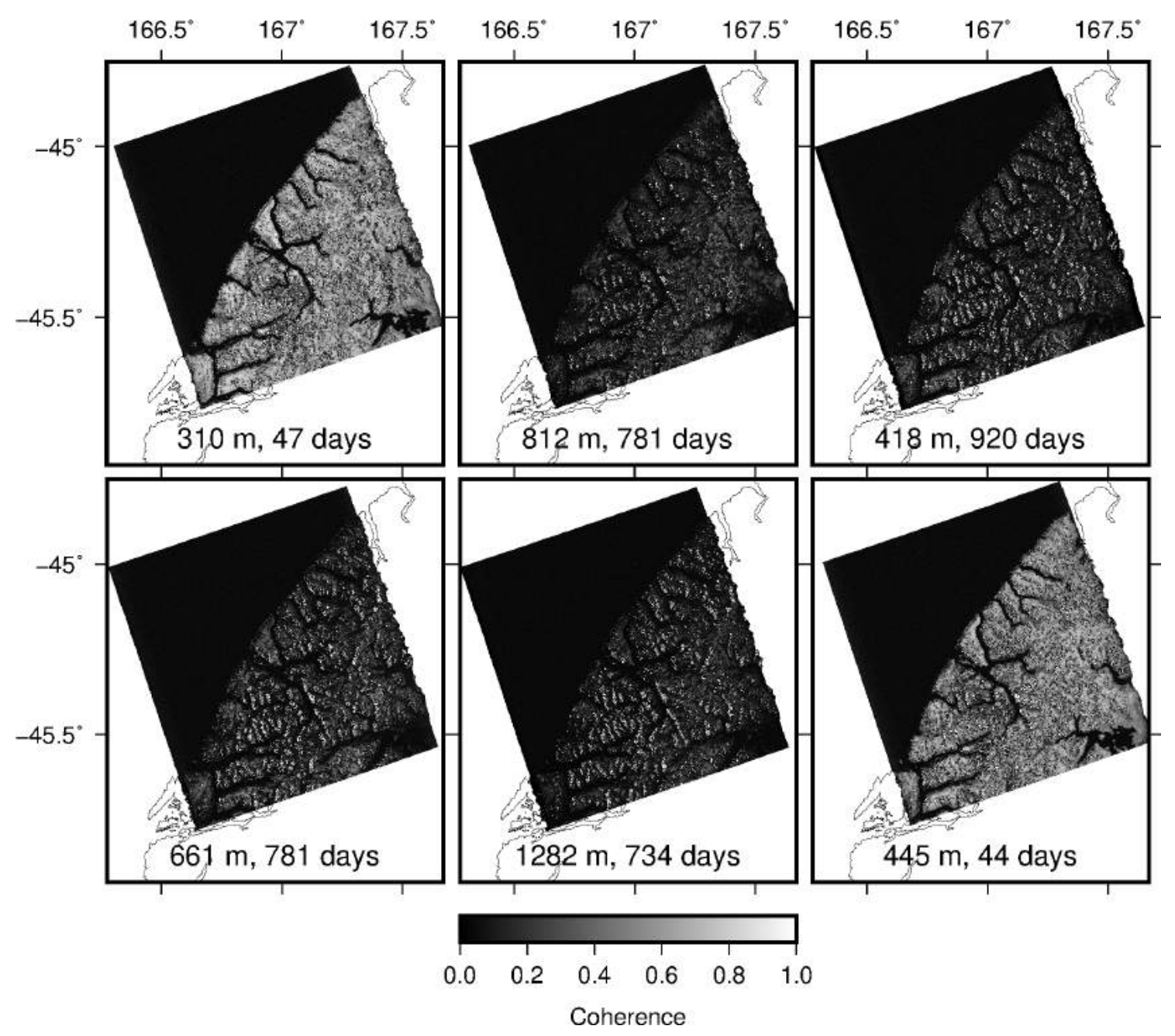

Figure 3.8: Comparison of coherence from summer-to-summer interferograms. Pairs with short-baseline and temporal separations have noticeably better coherence than those with long temporal and/or baseline separations.

separated by days to weeks tend to have higher coherence than those separated by months to years. Similarly, two SAR acquisitions separated by short perpendicular baseline separations $(<400 \mathrm{~m})$ tend to have higher coherence than those with longer baseline separations (c. $1000 \mathrm{~m})$. For pairs with both long temporal and baseline separations, coherence was poor. Overall, coherence values were less sensitive to seasonal change, than to baseline and temporal separations.

The network of interferograms constructed needs to be considered in detail based upon the intended use of the final product, and in order to generate a time series, all pairs of interferograms must be connected to each other. GMTSAR uses the SBAS approach (see Section 2.5), where the interferogram pairs are determined by the baseline distance separation and the temporal separation. In the ideal case, 


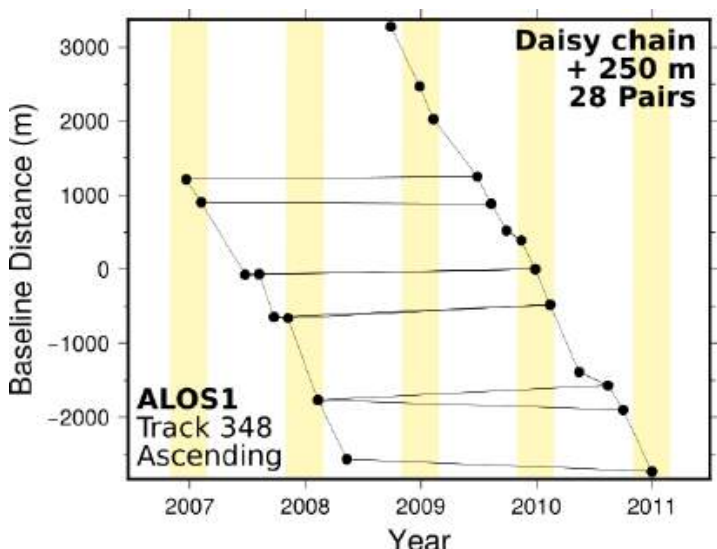

(a) Network 1: $B_{\perp}=250 \mathrm{~m}$

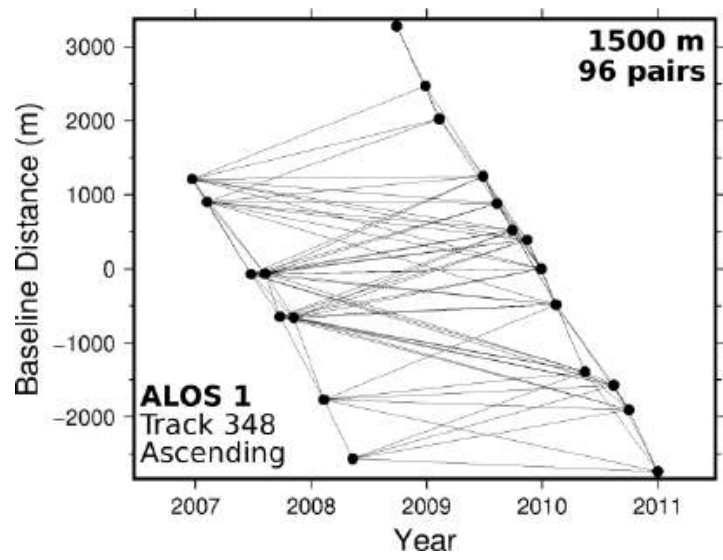

(c) Network 3: $B_{\perp}=1500 \mathrm{~m}$.

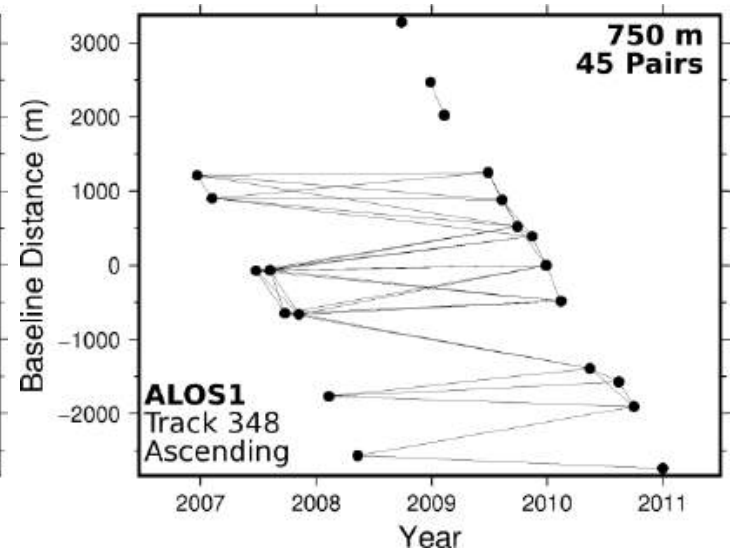

(b) Network 2: $B_{\perp}=750 \mathrm{~m}$.

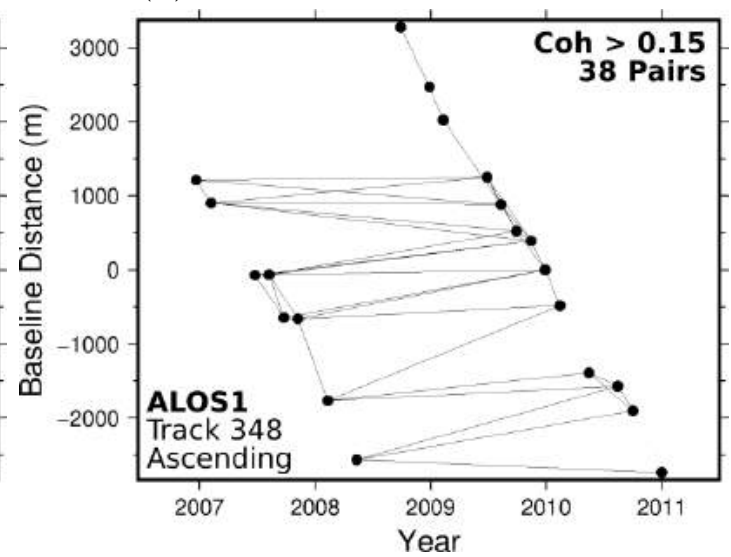

(d) Network 4: Coherence selection

Figure 3.9: Networks of interferograms tested during processing. Yellow lines in subfigure 3.9a denote summer months when snow cover and atmospheric water vapour typically is lowest.

a network would connect many interferograms with equal time spans and relatively short-baselines (Sandwell et al., 2011a). We present interferogram network testing completed with ALOS1 to determine the ideal network design for the given dataset.

\section{Network 1}

Network 1 was designed using selection criteria adapted from Simons and Rosen (2015) which required that each pair have a perpendicular baseline distance of less than $250 \mathrm{~m}$, not include scenes that were acquired during rain or snowfall events, and have a maximum temporal separation of less than 3.5 years. In Network 1, pairs with short $(250 \mathrm{~m})$ baseline separations were connected first [Figure 3.9a]. Preference was given towards images images acquired in the summer months, but due to orbital drift, the application of this criteria was relaxed. Next, the 'daisy 
chain' template (described in Section 2.3) was applied to connect pairs with short temporal separations. Within Network 1, all acquisitions were connected, with short and long temporal pairs and mainly short-baseline pairs. The pairs either side of the greatest baseline distance $(>6000 \mathrm{~m})$ were not linked in a circular manner to all other pairs. The total number of pairs was 28 , which is too few to generate a SBAS time series (Schmidt and Bürgmann, 2003).

\section{Network 2}

Sandwell et al. (2011a) suggest that the optimal baseline distance should be onequarter of the critical baseline distance. For ALOS1, this yields an optimal separation of c. $500 \mathrm{~m}$. For Network 2, the maximum baseline distance threshold was increased to $750 \mathrm{~m}$ as this was close to the optimal separation and connected most acquisitions. The total number of interferograms pairs increased to 45 . In this network, not all acquisitions are connected, with three acquisitions in late 2008 and early 2009 discarded. The largest baseline separation occurs in the months before the Dusky Sound earthquake, meaning that there were fewer interferograms constraining the deformation before the earthquake.

\section{Network 3}

In order to include data from all acquisitions, the maximum baseline separation was finally increased to $1500 \mathrm{~m}$, bringing the total number of interferograms to 96 . The baseline threshold was not extended further as visual inspection showed that a number of interferograms with baseline distances approaching $1500 \mathrm{~m}$ had extremely poor coherence. The total number of interferograms satisfied the SBAS requirement that the number of interferograms exceeded the number of SAR acquisitions and all but one acquisitions were connected via multiple loops. As a result of the increase in the number of interferograms spanning the date of the Dusky Sound earthquake, constraints on the earthquake displacements were highest for Network 3. 

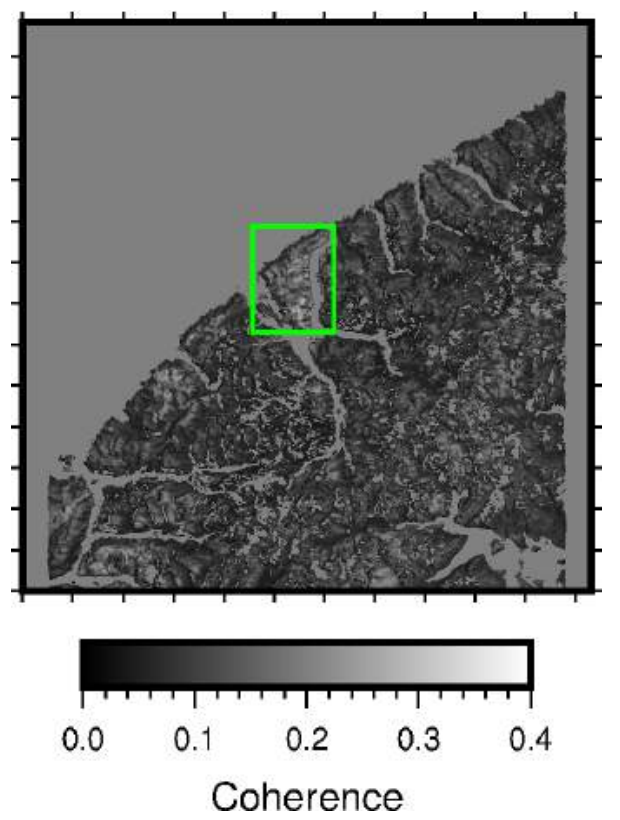

Figure 3.10: Region used to select interferograms with high coherence for Network 4 . If $50 \%$ or more of the pixels within the green box had a coherence greater than 0.15 the image was selected for the final interferogram network.

\section{Network 4}

Visual inspection showed that a high proportion of the interferograms from Network 3 had poor coherence. To remove these 'bad' pairs, the coherence selection method of Chaussard et al. (2015) was adapted and applied. Frames which containing greater than $50 \%$ of pixels with a coherence greater than 0.15 within the specified region of interest were selected for Network 4. Figure 3.10 shows the area of interest, which encompasses the region in which deformation from reactivated slip would be most pronounced around Secretary Island. For comparison, Chaussard et al. (2015) used a coherence threshold of 0.5: we lowered the coherence threshold, otherwise, no images would fulfil the criteria. This trade-off meant the number of images in the final network was still sufficient to complete time series analysis.

The coherence-based selection produced a network of interferograms in which each SAR acquisition was linked to all others, and the selected pairs all had moderate coherence across the region of interest. The maximum baseline separation was $1103 \mathrm{~m}$ but, not all pairs with separations less than this baseline distance were created. The benefit of processing all possible pairs first (i.e. Network 3 with $B \perp=1500 \mathrm{~m}$ ), and subsequently selecting based on coherence, is that interferograms with poor coherence were excluded. If included, the low-coherence interferograms would have add noise to the time series and weaken any deformation signal. 
Interestingly, some pairs with long-baselines (approaching c. $1300 \mathrm{~m}$ ) were retained as they had short temporal separations and coherence was maintained. These pairs would have been excluded with a simple baseline threshold as most interferograms would not have maintained coherence over that distance.

\section{Filtering}

GMTSAR uses an adaptive Goldstein-Werner filter and a Gaussian filter to remove phase noise, as described in Section 2.3. The amplitudes of both images, the real and imaginary components of phase, filter strength $(\alpha)$ and patch sizes are required. The default values for filter strength of $\alpha=0.5$ and patch size of $32 \times 32$ pixels were used in processing. Each patch was set to overlap the adjacent ones in the azimuth and range directions. Filter testing completed showed that the ideal Gaussian filter length given the noise level in the ALOS1 dataset was $400 \mathrm{~m}$ [Figure 3.11]. Shorterwavelengths resulted in increased unwrapping errors correlated with regions of poor coherence and data gaps. Longer-wavelength filters did not improve the phase signal but reduced the spatial resolution. When geo-coding the final image, the pixel size is reduced to one-quarter of the wavelength of the spatial Gaussian filter, so filter selection determines the final spatial resolution of the data. The application of a $400 \mathrm{~m}$ Gaussian filter reduced the unwrapping errors introduced by noise and did not degrade the resolution of the image unacceptably. Figure 3.11 (bottom) shows the difference between the original wrapped phase and $400 \mathrm{~m}$ filtered phase, to determine whether any of the deformation signal was removed in the filtering process. We find that some of the deformation signal is removed (faint fringes in Figure 3.11 bottom right), this is likely removed during Goldstein-Werner filtering in the frequency domain.

\section{Unwrapping Interferograms}

Unwrapping of interferograms was completed by Snaphu as addressed in Section 2.4. Parameters were set to: 1) not allow phase jumps across short distances; 2) 

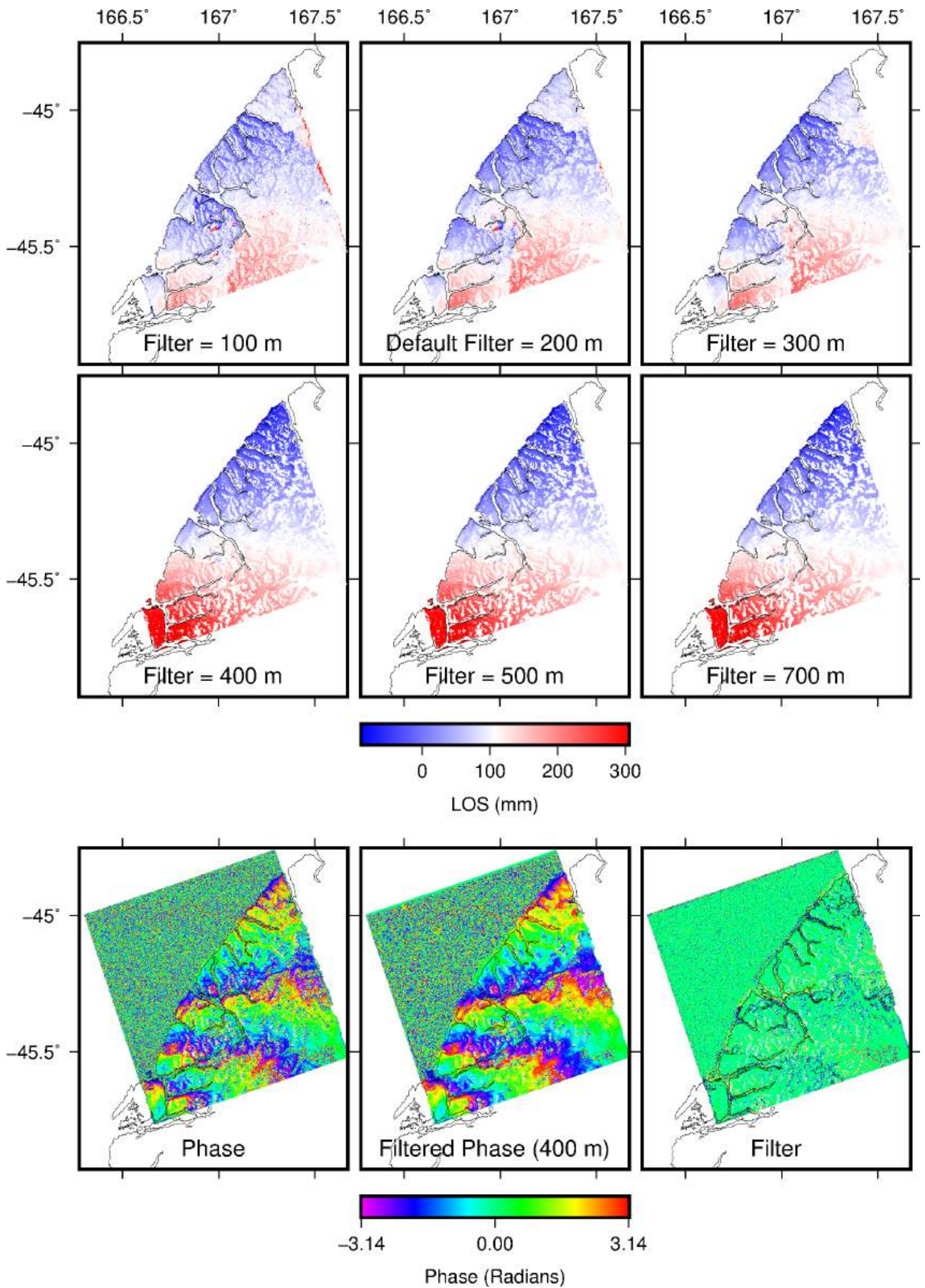

Figure 3.11: Top: Gaussian filter tests with increasing spatial wavelength. The filter wavelength varies from $100 \mathrm{~m}$ to $700 \mathrm{~m}$. Longer-wavelength filters $(400-700 \mathrm{~m})$ smooth across data gaps and produce interferograms with fewer unwrapping errors. Shorter-wavelength filters (100-300 m) have clear unwrapping errors where there are steps in the LOS across the scene. NB: Red (positive) indicates motion towards the satellite and blue (negative) indicates motion away. Bottom: The filtered phase (middle) was subtracted from the original phase (left) to determine how much of the deformation signal was being removed in the filtering process (right). 
determine the coherence values of pixels included in the unwrapped solution and; 3) apply an interpolation prior to unwrapping.

\section{Allowing Phase Jumps}

The deformation mode determines the number of phase jumps allowed within the unwrapped interferogram. A phase jump would be applicable for determining the displacement after an earthquake, where one side of the fault would have a path length several wavelengths greater than the other. When unwrapping this image, the step in phase needs to be preserved in order to capture the surface rupture. Here, we set this parameter to prohibit phase jumps and to smooth phase jumps across boundaries, as no surface ruptures, or features causing phase steps, were expected in this dataset.

\section{Coherence Masking}

Pixel selection based on coherence reduced the amount of phase noise in the unwrapped interferogram. Lower noise levels, reduced the number of unwrapping errors and computation time. Two modes of coherence masking were tested: first, a classic coherence threshold, and second, a uniform coherence mask.

A coherence threshold was tested to determine whether the phase value of a pixel should be used for the unwrapped solution based on its coherence. Low coherence pixels often relate to regions of water, vegetation growth or snow cover, and these regions add noise to the solution. The threshold excludes pixels with a coherence below the specified threshold. Figure 3.12 shows the speckle effect from pixels with low coherence. When the threshold is 0.05 , the image contains patches where the reflected signal has caused a speckled effect over water bodies and mountain tops. At a threshold of 0.3 there is no speckle in the image, but useful data have been omitted. A threshold of 0.15 still retains some speckling, but the spatial coverage of the data is better. Pixels remaining in regions of water were omitted using a land/water mask.

Network 1 (see Figure 3.9a) was unwrapped using a coherence threshold 

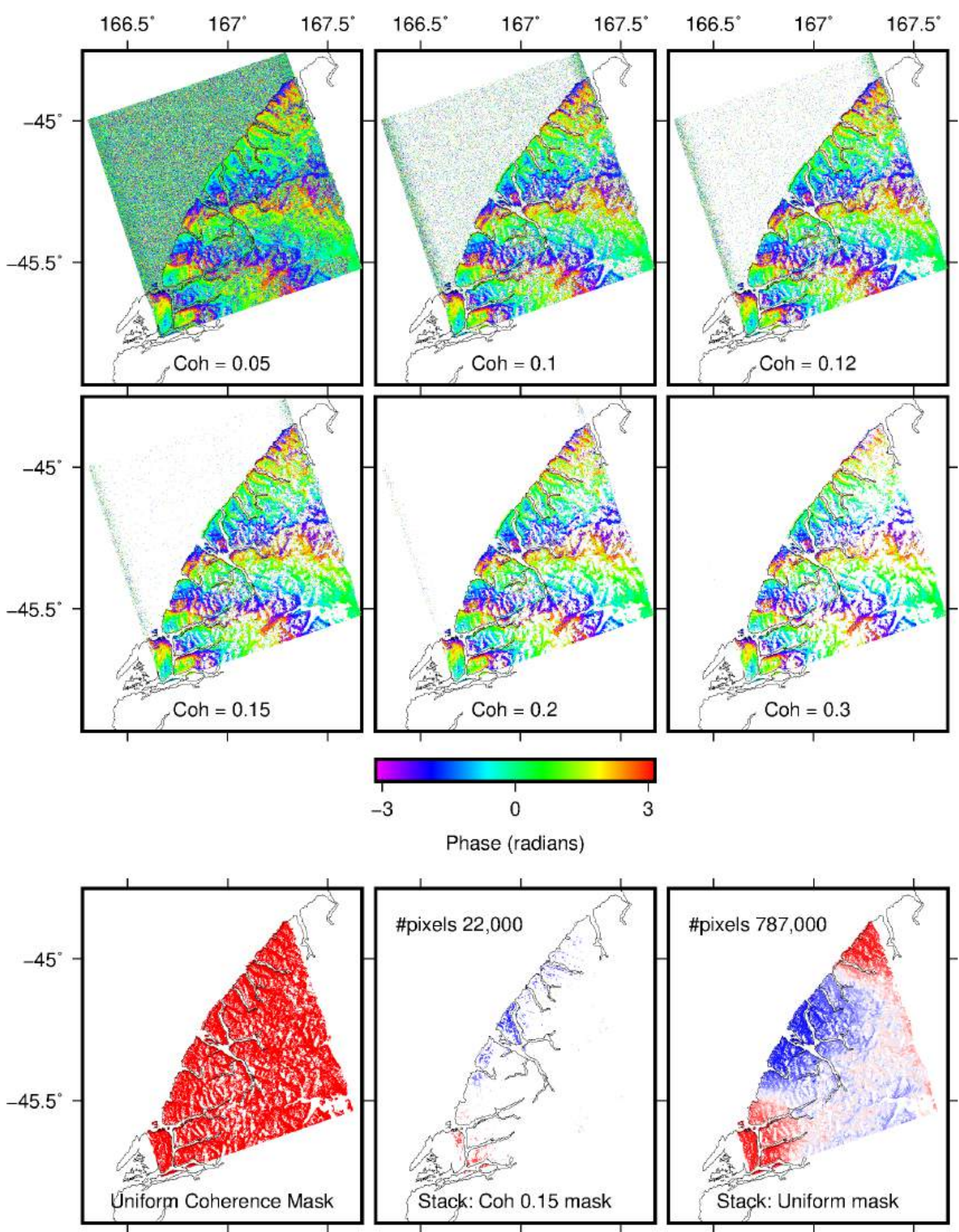

Figure 3.12: Coherence mask testing. (Top) Pixels with a coherence value below a certain threshold (0.05-0.3) were excluded from unwrapping. (Bottom left) Example of a uniform coherence mask. (Bottom centre) Stack of interferograms unwrapped with a pixel coherence threshold of 0.15. (Bottom right) Stack of interferograms unwrapped with a uniform coherence mask. 
of 0.15. Interferograms with large baseline separations had sizeable regions of low coherence, this resulted in spatially disconnected phase values, and unwrapping errors occurred across these data gaps. Additionally, if a pixel was not present in every interferogram, this pixel was dropped when stacking. Figure 3.12 (bottom centre) shows the few pixels that were coherent through time with a spatial coherence threshold of 0.15 . Only pixels along the coast with consistently high coherence were retained in the final stack.

To improve the number of available pixels for stacking and time series analysis, a uniform coherence mask was applied. The coherence mask was generated by stacking the coherence grids from all interferograms, and then pixels with coherence below 0.09 were removed [Figure 3.12 (bottom left)]. This uniform coherence mask was applied to all wrapped interferograms so that each unwrapped interferogram had exactly the same pixels present. Through the application of a uniform coherence mask, interferograms with relatively higher coherence had viable pixels excluded and interferograms with relatively low coherence had noisy pixels included. The introduction of these noisy pixels was the trade-off for having an adequate number of temporally coherent pixels for time series analysis [Figure 3.12 (bottom right)].

\section{Interpolation Method}

Unwrapping errors were pervasive when no interpolation was applied prior to unwrapping [Figure A6]. The quantity and spatial extent of the unwrapping errors made manual correction untenable for the full dataset of 96 interferograms (Network 3). When unwrapping interferograms from both ALOS1 and Sentinel 1A/B without interpolation, there were some interferograms which took days to unwrap and were eventually abandoned. These particular frames were characterised by large data gaps and the inclusion of noisy pixels, resulting from large regions of poor coherence inland along mountain ranges where the highest levels of rainfall are recorded [Figure 3.3].

The nearest neighbour interpolation method from Shanker and Zebker (2009) was implemented with Snaphu to reduce processing time, reduce unwrapping errors 
and allow for unwrapping across regions of low coherence (as explained in Section 2.4). Applying the interpolation meant that noisy pixels with low coherence could be unwrapped, and unwrapping errors across data gaps were reduced. With the application of interpolation, all ALOS1 interferograms could be unwrapped and a higher proportion of Sentinel 1A/B interferograms were completed. ALOS2 generally had high coherence and fewer unwrapping errors so the implementation of the interpolation made little difference to the result. The pre-unwrapping interpolation reduced processing time by up to a factor of ten for ALOS1 data. For track 348, frame 6260 , the coherence was higher along the coast and around rocky outcropping mountain tops [Figure 3.13 (left)]. High-coherence regions are likely to have been sampled more frequently, have fewer interpolated values and be an accurate representation of the deformation field. In regions with poor coherence, fewer 'real' data points exist, more values were interpolated and the deformation field may have been under-sampled or biased by the noisy phase values.

The best results were achieved when a uniform mask was applied to all interferograms, the unwrapping coherence threshold set to 0.001 and the nearest neighbour interpolation method of Snaphu was applied. All remaining pixels were forced to be unwrapped. For the most part, unwrapping errors occurred in small isolated pockets in which coherence was particularly poor. Mostly unwrapping across data gaps from fjords and other water bodies was completed correctly without error. For interpolated unwrapped images, the application of a coherence threshold or uniform coherence mask produced deformation fields with the same amplitude and spatial distribution. Data with the uniform coherence mask applied, included more noisy pixels, but resulted in the same deformation field and maintained an adequate number of pixels for time series analysis.

\section{Noise Corrections}

Figure 3.14 presents examples of the ionosphere and orbital noise in ALOS1 and ALOS2 interferograms, and atmospheric noise in Sentinel 1A/B. A strong deformation gradient is present across ALOS1 and ALOS2, and a scaly texture appears in 


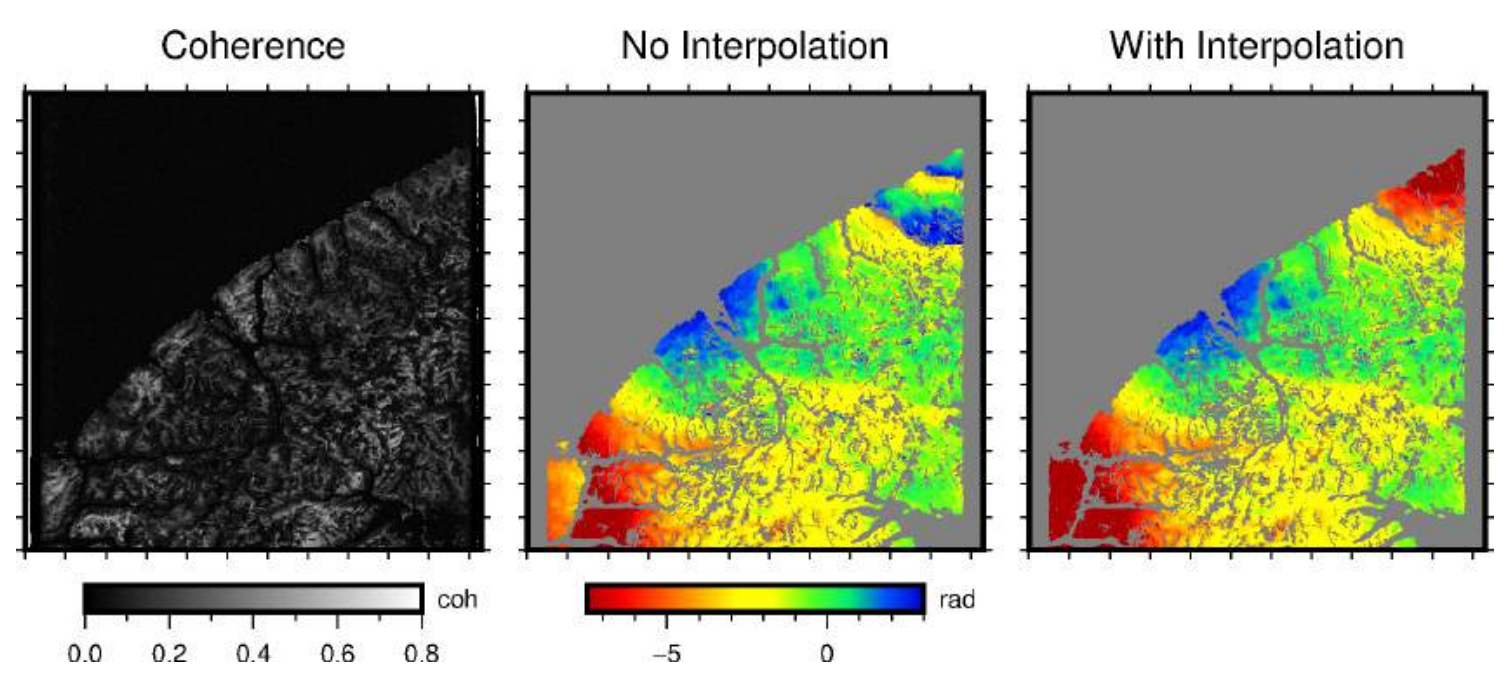

Figure 3.13: Interferograms unwrapped without interpolation (centre) and with interpolation (right). Coherence plot (left) shows the regions with higher coherence exist along the coast and lowest on the mountain tops parallel to the coast. These SAR images have a baseline separation of $645 \mathrm{~m}$ and temporal separation of 2.55 years. Note the unwrapping errors (centre). The colour in the top right corner jumps from yellow to blue over a fjord, indicating a step from c. -7.5 to c. 3 radians of LOS displacement. As we know there was no surface rupture during the epoch of the interferogram, this step must be an unwrapping error. A similar feature can also be seen in the bottom left corner. In the interpolated image (right) this step does not exist and the phase gradient is continuous across the whole image.

the Sentinel 1A/B example. The ramp in ALOS1 and ALOS2 is attributed to a combination of long wavelength ionosphere noise and residual orbital errors. The atmospheric noise in Sentinel 1A/B is attributed to lenses of variable air pressure and water vapour content, causing localised propagation delays, as seen in Figure 3.14 .

\section{Long-Wavelength}

Interferograms were detrended to remove the long wavelength noise (as described in Section 2.4). As the system was under-determined, a least squares solution was used to solve for the set of linear parameters which described the linear ramp, where the azimuth and range gradients were calculated. The calculated ramp was then subtracted from the data and the process repeated for each unwrapped interferogram [Figure 3.15]. An issue with removing a linear ramp is that if there is deformation from a large earthquake some distance away (e.g. $>50 \mathrm{~km}$ ), then the deformation field may approximate a ramp at the scale of the InSAR frame. 

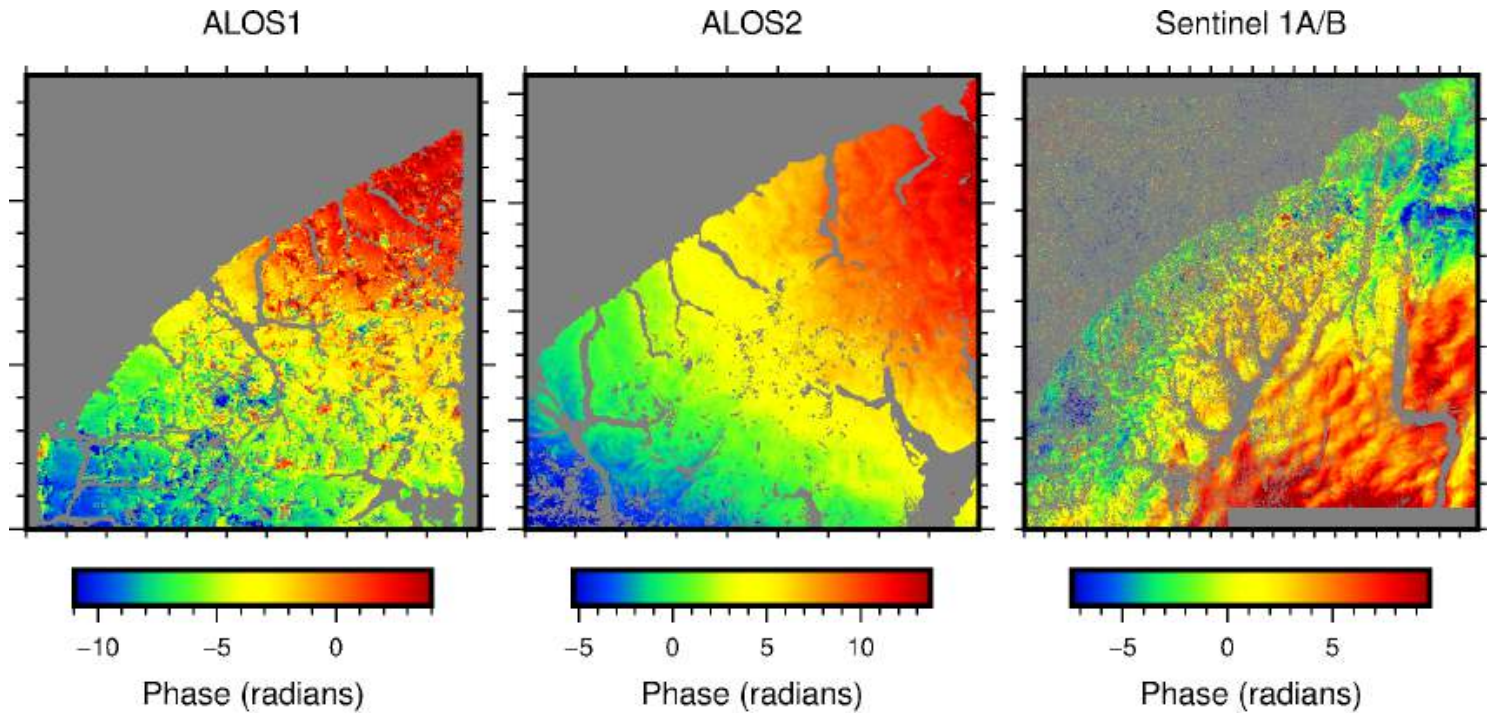

Figure 3.14: Examples of noise from ionosphere (ALOS1: 21 Dec 2006 - 8 Aug 2007, ALOS2: 25 Nov 2015 - 3 Feb 2016) and atmosphere (Sentinel 1A/B: 3 Feb 2017 - 27 Feb 2017). The epochs of these interferograms do not contain any large scale tectonic deformation so unwrapped phase change has been attributed to noise in the data. ALOS1 and ALOS2 interferograms have strong gradients (or linear ramps) from the bottom right to top left of the frame, indicative of ionosphere noise. The interferogram from Sentinel 1A/B exhibits a scale-like texture from localised changes in atmospheric conditions.

Unwrapped Interferogram

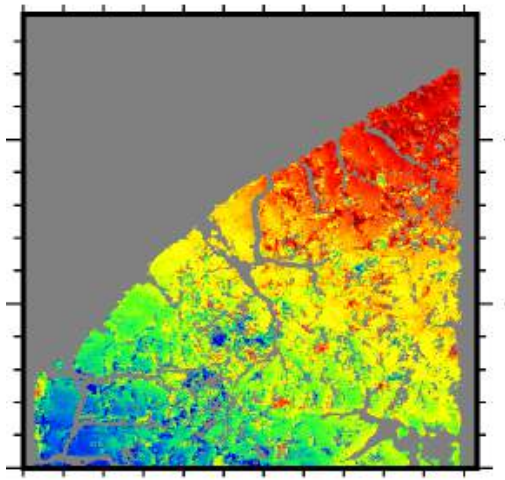

Calculated Trend
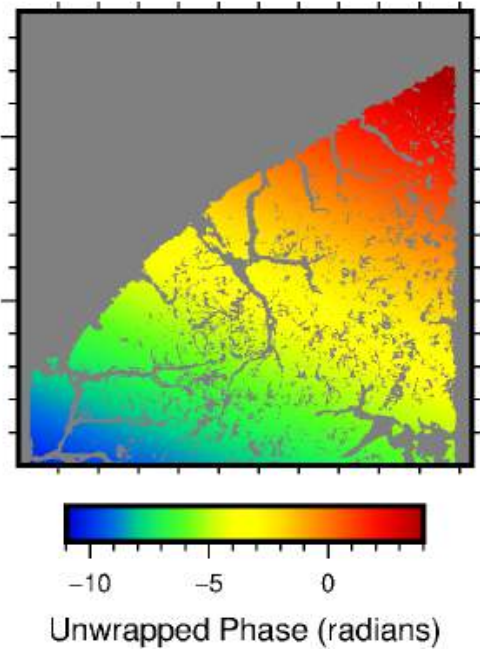

Detrended Interferogram

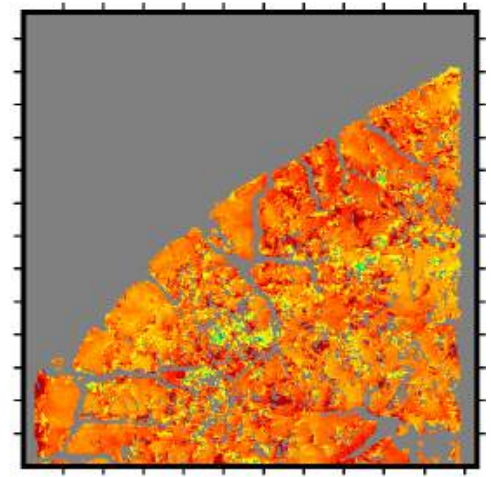

Figure 3.15: The linear trend for each interferogram was calculated and subtracted from the data to produce a detrended interferogram. The calculated linear trend approximates the residual orbital noise and long-wavelength ionosphere noise in the unwrapped interferogram so this can be removed from the final solution. 

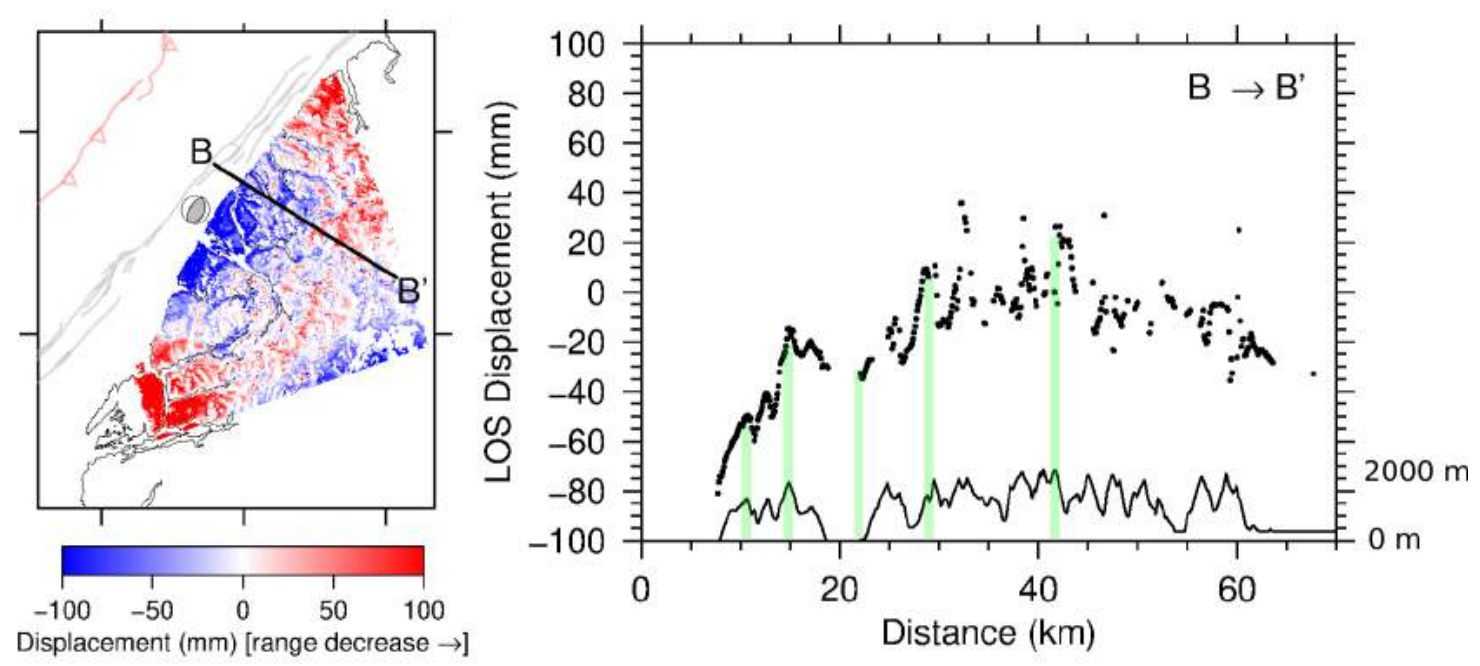

Figure 3.16: Comparison of topography and residual noise field for interferogram from track 348 frame 6260, 28 June 2009-13 August 2009. The residual noise field is topographically correlated. Gaps in data represent the 'shadow' regions where due to the steepness of the topography the emitted radar signal does not reach [see Figure 2.2].

\section{Short-Wavelength}

The SRTM 30 m DEM was used to solve for topographically correlated short-wavelength noise. A linear function with elevation was assumed and a least-squares linear inversion performed to solve for the best-fitting stratified delay for the observations (Hamling and Hreinsdóttir, 2016). Figure 3.16 shows the residual deformation field after detrending and the correction for topographically correlated noise was applied. short-wavelength topographically correlated noise is still present in the data, this remaining short-wavelength noise was corrected for by brute stacking.

Stacking was completed by summing all detrended and unwrapped interferograms and dividing by the cumulative time. This enhanced the SNR by reducing the contribution from residual noise in the dataset. A linear deformation rate was produced for the time period of the interferograms. The ideal case would have been to stack all interferograms before the George Sound earthquake, between the George Sound and Dusky Sound earthquakes, and after Dusky Sound earthquake to determine the linear rates between each event and determine the magnitude of deformation from reactivated slip. However, due to the orbital drift of ALOS1 and the temporal resolution of the acquisitions, few interferometric pairs were available 
with which to separate out the deformation associated with each event.

Figure 3.17 compares the results from stacking 'all', 'good' and 'bad' interferograms from the adapted coherence-based selection method of Chaussard et al. (2015) (see Section 3.3). 'All' includes the full set of interferograms from Network 3, 'Good' is all interferograms in Network 4 and 'Bad' is interferograms from Network 3 which were excluded from Network 4. Figure 3.17 highlights the impact of pixel coherence and detrending on the magnitude of the stacked deformation signal. Where low-coherence interferograms were included, the deformation signal centred on Secretary Island had a lower magnitude and spatial extent. When stacking the high-coherence and detrended interferograms, the magnitude of the deformation signal increased from c. 1 radian to $>2$ radians [Figure 3.17, middle right]. In all cases, the speckled texture from short-wavelength noise has been removed from almost all regions.

\subsection{Short Baseline Subset (SBAS) Time Series}

The SBAS method of Schmidt and Bürgmann (2003) was used to solve for cumulative LOS displacements and linear velocity for the period 2007 to 2011. The SBAS method requires all interferograms to be fully connected to the network. As there are some pairs within Network 4 that did not complete loops, these pairs were redundant and could not be included in the time series inversion. Grids of root mean square (RMS) values and DEM errors were produced from the SBAS inversion. Typically this method requires interferograms to be tied to a stable point within the frame (Schmidt and Bürgmann, 2003). From GPS data, we know that the Dusky Sound earthquake caused displacements across all InSAR frames. Consequently, there were no stable points in any frame for the interferograms to be tied to. If there had been a cGPS station in each of the frames, those data could have been used to tie interferograms and produce an absolute time series of deformation.

The atmosphere correction method of Tymofyeyeva and Fialko (2015) was tested on the dataset during SBAS time series analysis. This correction method 

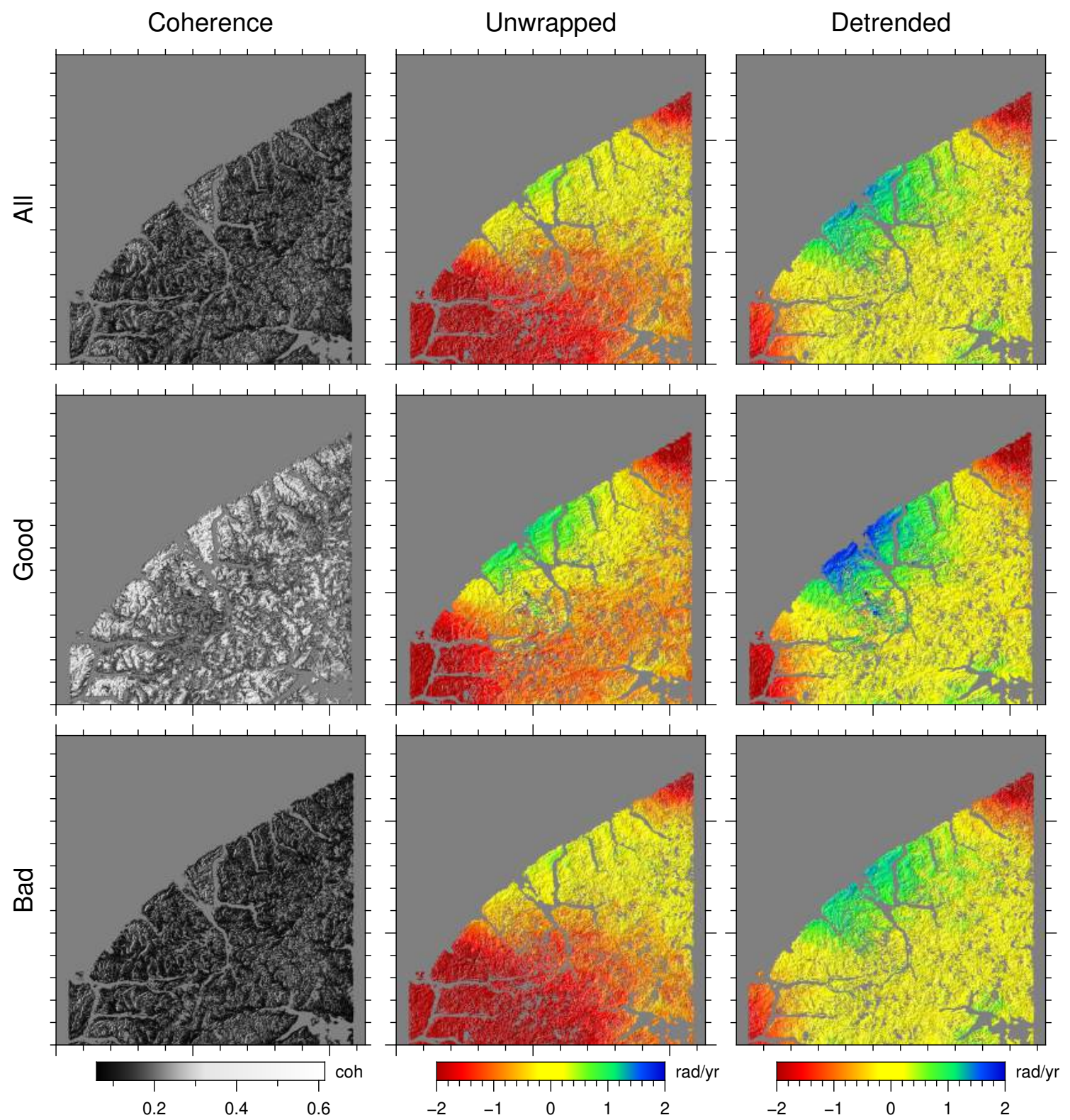

Figure 3.17: Stack of 'all' interferograms (96), 'good' interferograms which were above the coherence threshold (36), and 'bad' interferograms which were below the coherence threshold (60). The coherence, unwrapped and detrended unwrapped interferograms have been stacked for each group. The stack of 'good' interferograms has greatly improved coherence, and includes the highest magnitude deformation signal around Secretary Island. NB: The colour palette has been clipped to values in the 'good' 'detrended' stack. 
yielded unsatisfactory results as the algorithm assumes that the residual signal after completing the circle test is from atmospheric contribution alone rather from than tectonic signals and so smooths the residual through time. The application of this temporal smoothing factor smeared the co-seismic signal from the Dusky Sound earthquake throughout the time series.

Figure 3.18 presents the linear velocity maps for the parameter tests completed. The difference in final velocity indicates how sensitive the final result is to the tested parameter. We find that noise levels are reduced when all 96 interferograms from Network 3 were included, when PS pixels are weighted and when higher-resolution processing is completed. The noise field is most sensitive to the network of interferograms used, which indicates that the inversion result is more robust with a greater number of interferograms, irrespective of the coherence values. The spatial distribution of noise appears to be correlated to regions with low coherence, but not correlated to the long-wavelength deformation signal. We find that the amplitude of the deformation signal is not altered by the application of these processing steps (mean velocity value varies by $\pm 1 \mathrm{~mm} / \mathrm{yr}$ between tests). This gives confidence that we are only improving the noise level without degrading the signal.

\subsection{Modelling}

We adapted the method of Hamling and Hreinsdóttir (2016) to isolate surface deformation associated with a triggered slip source near to Secretary Island following the Dusky Sound earthquake. We used geodetic modelling to test whether reactivated slip on a patch similar to the 2003 Secretary Island earthquake source could result in a deformation field similar to that observed in interferograms. To achieve this an accurate model of deformation associated with the 2009 Dusky Sound earthquake was produced, so the associated deformation field could be removed from the observations. Any residual localised deformation centred on Secretary Island was then examined for evidence of reactivated slip. The deformation modelling involved four sequential tasks: 1) forward modelling of potential Secretary Island slip; 2) Bayesian 


\section{Network 4 (36 intf) / No PS weighting / Low resolution}
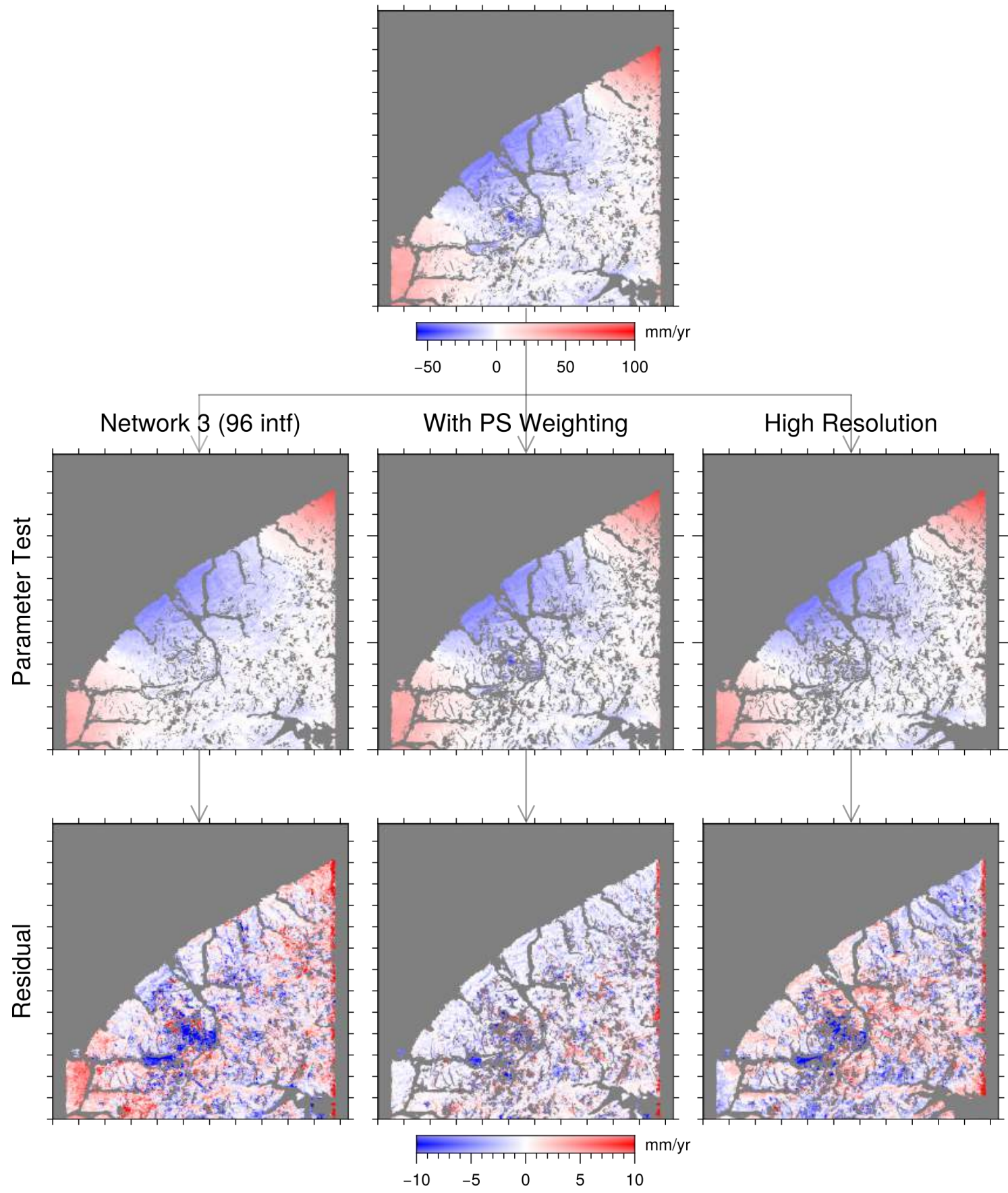

Figure 3.18: Comparison of SBAS results. Velocity grids from each parameter test were subtracted from the 'control' velocity grid (top) to calculate the residual (i.e. the difference between the velocity fields derived with and without weighting for PS). The residual velocity grids show that the different parameters only improve the noise level and do not change the amplitude of the long-wavelength signals. 
modelling of the Dusky Sound earthquake; 3) removal of Dusky Sound deformation from the interferograms and; 4) modelling the residual signal around Secretary Island. The results of the modelling are shown in Chapter 4, and compared with Beavan et al. (2010) in Chapter 5.

\section{Forward Model of slip below Secretary Island}

Forward modelling was completed as a first approximation to compare the hypothesised reactivated slip signal from below Secretary Island to the observed deformation pattern (step 1). A Secretary Island source was modelled as a single fault plane undergoing uniform slip a homogeneous elastic half-space using the method of Okada (1985). The fault geometry parameters for the 2003 Secretary Island earthquake obtained by Reyners et al. (2003) were adapted to visually match the observed surface deformation around Secretary Island, following the 2009 Dusky Sound earthquake. The magnitude of slip from the 2003 Secretary Island earthquake was reduced from a maximum slip of $2.4 \mathrm{~m}$ to $1.0 \mathrm{~m}$ to align the magnitude of reactivated slip with that of Hamling and Hreinsdóttir (2016) at George Sound.

\section{Bayesian Model of Dusky Sound}

We next generated a Bayesian statistical model to determine the best-fitting parameters for the Dusky Sound earthquake, using posterior probability density functions (PDFs) calculated with GBIS (Bagnardi and Hooper, 2018) (step 2). Dusky Sound modelling was completed with ALOS1 data from tracks 348, 349 and 639. The data were collected at different time intervals following the earthquake so contain increasing amount of post-seismic deformation: track 349 on acquired on 15 July 2009 (the day of the earthquake), track 639 was acquired 8 days after and track 348 acquired 28 days after the Dusky Sound earthquake. The closest cGPS stations to the study area are those at PYGR and Mavora Lakes (MAVL) which are both c. $100 \mathrm{~km}$ from Secretary Island [Figure 1.1]. A total of 41 cGPS and sGPS sites were included in the inversion, which provided absolute values of deformation for 
the region south of Hokitika. The cGPS data were acquired on 15 July 2009 and the sGPS data acquired after the earthquake on 19-21 August 2009. The sGPS dataset therefore includes some post-seismic deformation signal; without these additional far-field data, the inversion was found not to converge.

In preparing the InSAR data for modelling, pixels with coherence less than 0.15 were masked and the image was sub-sampled to a pixel size of $100 \mathrm{~m}$. Quad-tree sub-sampling was used to reduce the total number of data points (as described in Section 2.6). A quad-tree variance threshold was imposed to determine the amount of variance allowed within one cell. This parameter was varied until the final number of data points was c. 1000 [Figure 3.19]. The quad-tree sub-sampling algorithm could not discriminate between short-wavelength noise (i.e from unwrapping errors) and the deformation signal of interest, as sub-sampling is sensitive to any phase gradients. This led to over-sampling in noisy regions and under-sampling in low-noise regions.

The interferograms were tied to the GPS co-seismic displacements published by Beavan et al. (2010). The appropriate look vectors were calculated with the the incidence $(\delta)$ and azimuth $(\gamma)$ angles:

$$
\begin{aligned}
& \operatorname{look}_{E}=-\cos (\gamma) \times \sin (\delta) \\
& \operatorname{look}_{N}=\sin (\gamma) \times \sin (\delta) \\
& \operatorname{look}_{U}=\cos (\delta)
\end{aligned}
$$

Each GPS component was multiplied by the equivalent component of the look vector in order to project the north, east and up GPS measurements into a LOS displacement vector:

$$
G P S_{L O S}=\left(G P S_{E} \times l o o k_{E}\right)+\left(G P S_{N} \times l o o k_{N}\right)+\left(G P S_{U} \times l o o k_{U}\right)
$$

A static shift was then applied, whereby the difference between the LOS vector at one cGPS station (i.e. PYGR) and the equivalent location (averaged over $300 \mathrm{~m}$ of the GPS station location) was subtracted from the whole interferogram. 


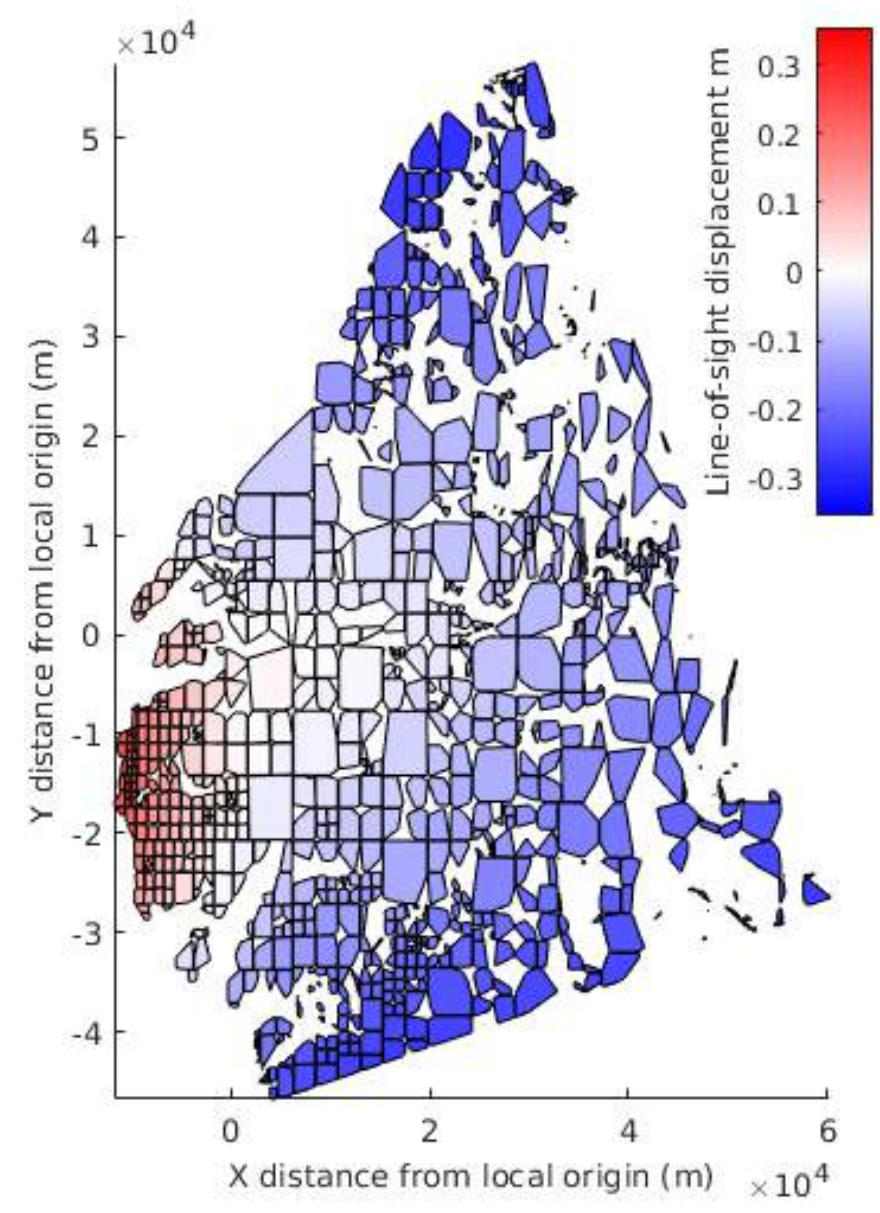

Figure 3.19: Quad-tree sampling for Dusky Sound modelling. Track 349, frame 6250 had c. 500,000 pixels, which were sub-sampled with the quad-tree sampling method to 1006. The density of measurements increases towards the epicentral region as the phase-gradient steepens. 

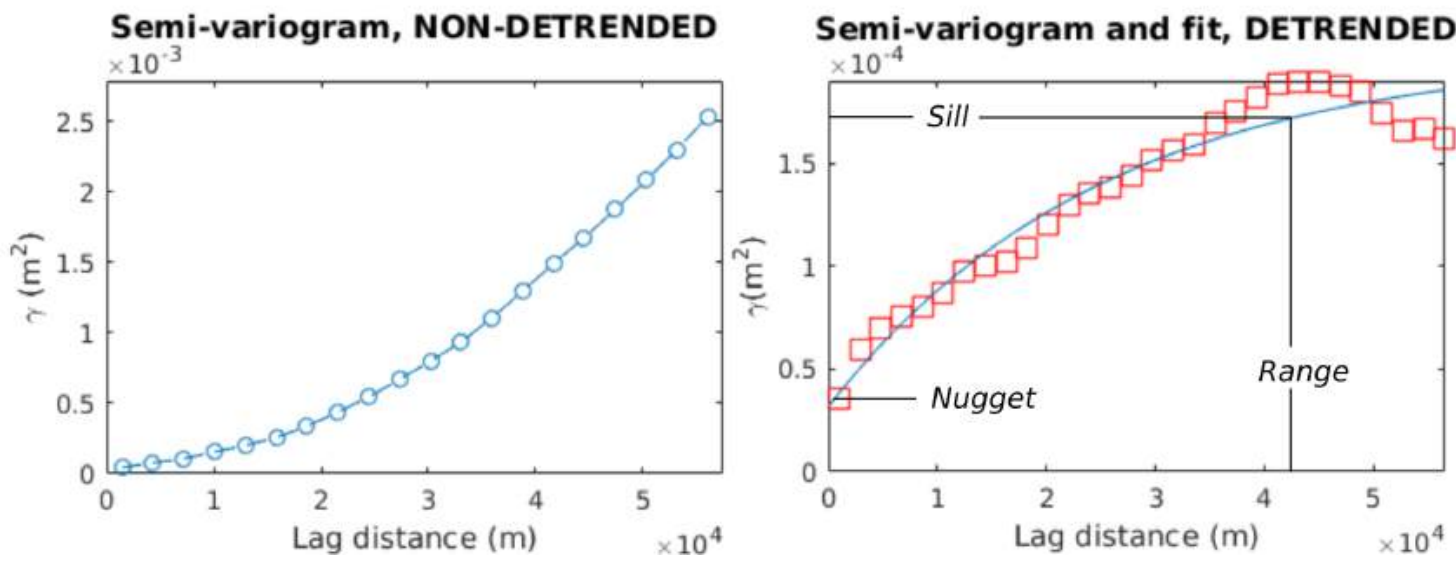

Figure 3.20: Variogram for track 639 used to estimate noise. Sill $\left(1.7214 x 10^{4} \mathrm{~m}^{2}\right)$, Range $(25214.92 \mathrm{~m})$ and Nugget $(3.1907 e-05 \mathrm{~m})$ values calculated from the semivariogram.

Errors within the interferograms were measured directly in GBIS by calculating the sill, range and nugget values for each frame, and then generating a semi-variogram (as described in Section 2.8). Noise was assumed to have a Gaussian distribution and to be the same across deforming and non-deforming regions. Bagnardi and Hooper (2018) recommended that sill, nugget and range values are calculated over a non-deforming region at least as large as the deformed region (Bagnardi and Hooper, 2018). As the Dusky Sound earthquake caused displacement across all frames, we used the northern most frame along track 639, $100 \mathrm{~km}$ from the epicentre of the Dusky Sound earthquake, to estimate spatial noise [Figure 3.20]. Noise levels for this frame were estimated and applied to the other frames along the same track acquired on the same days. For tracks 348 and 349, the sill, nugget and range values were calculated in a region to the southeast of the Dusky Sound epicentre, where the deformation gradient was lowest. Any residual longwavelength noise was solved for as a linear plane during the inversion.

Initial parameters were assigned from seismological modelling of the Dusky Sound of slip distribution from Fry et al. (2010). As some geological information is available, such as the surface trace of the plate boundary, a priori bounds can be placed each source parameter. The bounds of each parameter were varied until a full spectrum of geologically reasonable parameters were tested and the PDF each had a Gaussian distribution. The input bounds were limited as follows; length 10- 


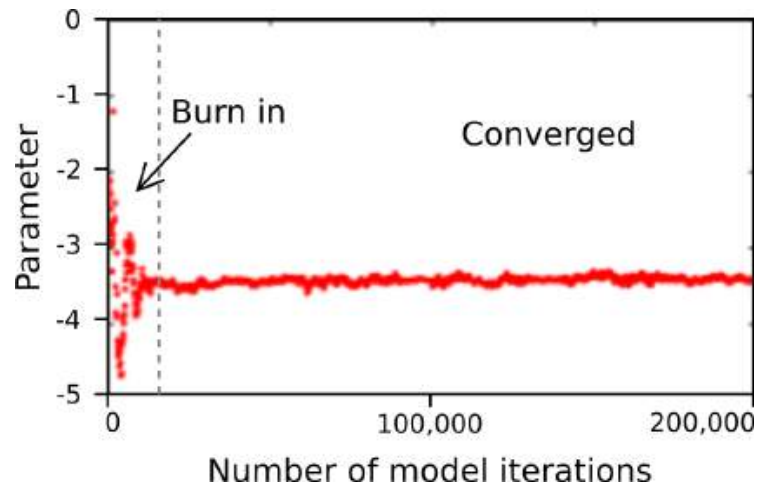

Figure 3.21: Example of model parameter converging over 200,000 iterations. The values in the 'burn' period are disregarded and those once the value has 'converged' are retained in the joint PDF's.

$120 \mathrm{~km}$, width $1-60 \mathrm{~km}$ depth to bottom edge of fault plane $10-50 \mathrm{~km}$, dip $0-90^{\circ}$, strike $-180-180^{\circ}$, magnitude of strikeslip -20-20 m and magnitude of dipslip -20$20 \mathrm{~m}$ and a Poisson's ratio of 0.25 was set. The Bayesian model solved for and removed a constant offset and linear ramp in the InSAR data, and for fault length, width, depth, dip, strike, location, strike-slip and dip-slip. To assess model results, convergence plots were visually inspected to check for parameter convergence and the posterior PDF were inspected to check for Gaussian distribution [Figure 3.21, 4.12a and 4.12b]. The GPS and InSAR data and optimal modelled values were compared and the residual values calculated. When each parameter converged, the PDF's had a Gaussian distribution and the residual values were below $0.2 \mathrm{~m}$.

\section{Isolating the Secretary Island Local Deformation Field}

We adapted the method of Hamling and Hreinsdóttir (2016) to isolate any deformation signal near Secretary Island. To isolate deformation signal near George Sound following the Dusky Sound earthquake, Hamling and Hreinsdóttir (2016) subtracted the Dusky Sound deformation model of Beavan et al. (2010), solved for and removed a linear plane and topographically correlated noise. Hamling and Hreinsdóttir (2016) applied these corrections to the cumulative displacement field for the nine months following the Dusky Sound earthquake.

As we do not have GPS data for the period following the Dusky Sound earthquake, we instead selected an unwrapped interferogram containing the local deformation signal which aligned with the dates of the sGPS campaign following the Dusky Sound earthquake. The unwrapped interferogram from this time period 
was shifted to align with the co-seismic offset recorded at site DF4L (method as described above). The Dusky Sound model produced in this study was then subtracted from the GPS aligned interferogram (step 3). The remaining deformation field was detrended by solving for the best-fitting linear plane and the short-wavelength, topographically correlated noise, was removed. The location of the Secretary Island deformation model was shifted laterally by trial and error through fault locations along strike and updip from the Secretary Island epicentre. Once the spatial pattern of modelled deformation matched that of the deformation field, the amount of slip was solved (step 4).

Two methods were then applied to determine the magnitude of slip. Our first approach was to visually match cross-section profiles through the observations and models. We then increased the modelled slip in the best-fit location, to determine the magnitude of the slip given the noise level of the data. This was repeated until the modelled surface deformation visually matched that of the observations, i.e. when the shape of the modelled profile matched that of the observations. Our second approach used a least-squares linear inversion to determine the best-fit magnitude of slip. The resulting slip value was that which produced the lowest misfit between the observations and the model. This inversion assumed that all fault parameter were known and the only unknown variable was that of the magnitude of slip. Histograms of the residual values at each step compared to determine whether the models improved the fit to the data, introduced signal to the data, or if the difference was below the standard deviation of the observations.

\subsection{Summary}

Data from ALOS1 were batched processed as follows. Raw SAR frames were preprocessed and focused using precise orbit information. The SRTM $30 \mathrm{~m}$ DEM was transformed to radar coordinates and the appropriate topographic shift was calculated. Pixels with persistently high amplitudes were up-weighted and SLC's were re-calculated. Many slave images were aligned to a single master, and each pair in 
Network 3 were used to generate interferograms with the topographic and orbital contribution of phase removed. An adaptive Goldstein-Werner filter and a $400 \mathrm{~m}$ Gaussian filter were applied to the wrapped phase to remove short-wavelength noise. A uniform coherence mask was generated to force the same pixels in each image to unwrap. The phase of each interferogram was unwrapped with Snaphu interpolation to produce a map of relative ground displacement for the given epoch. Each unwrapped interferogram was detrended to remove residual long-wavelength noise from ionosphere delays and residual orbital errors. ALOS2, and co-seismic ALOS1 interferograms were processed with two-pass processing, which only varies from the process above in that not all frames are aligned to a single master image, pixels were not weighted, and a coherence threshold of 0.12 was applied prior to unwrapping.

Data from ALOS2 and the ALOS1 images of the co-seismic offset of Dusky Sound were processed by the two-pass method with the same key steps of SAR focusing, alignment, interfering, unwrapping and noise corrections [Figure 3.4]. In the end, processing with Sentinel 1A/B data were abandoned after satisfactory unwrapping could not be achieved. Owing to the short wavelength, the coherence was particularly poor in the vegetated regions, and even with the implementation of interpolation, not all interferograms could not be unwrapped. Additionally, the interferograms which could be unwrapped included sizeable local atmosphere delays, which swamped any tectonic deformation within the frame [Figure 3.14].

Images from ALOS1 (track 348, frame 6260) were stacked and an SBAS inversion performed to calculate the cumulative displacement at each acquisition date. Bayesian modelling using a uniform slip, single-fault model was completed to model and subsequent remove the deformation field from the Dusky Sound earthquake. Finally, a potential fault source on the plate interface below Secretary Island was modelled and the amount of required slip determined.

This chapter has described the dataset and sequence of analytic steps used in this study, the results are presented in the following chapter. 


\section{Results}

This chapter presents results from Interferometric Synthetic Aperture Radar (InSAR) processing, time series analysis and modelling. A network of interferograms was processed to generate a time series of deformation with the intention of determining whether the rupture patch of the 2003 Secretary Island earthquake was reactivated by the 2009 Dusky Sound earthquake. The localised surface deformation signal around Secretary Island was then isolated by removing the surface deformation associated with the 2009 Dusky Sound earthquake and detrending the residual. The remaining local surface deformation was modelled with a local source near to the 2003 Secretary Island rupture surface. We cannot rule out reactivated slip in the vicinity of Secretary Island following the Dusky Sound earthquake.

For clarity, InSAR results are presented in line-of-sight (LOS) geometry in both radians and millimetres. The convention used for presenting InSAR results is that LOS motion away from the satellite is a positive number in radians and negative number in millimetres, and, vise versa, LOS motion towards the satellite is a negative number in radians and a positive number in millimetres. As presented in this chapter, the Dusky Sound earthquake moved the ground surface south-west and down, bringing the ground surface closer to the satellite on ascending tracks (348 and 349), and away from the satellite on descending tracks (639). Throughout the chapter, we refer to the 'local deformation signal', this is the region of motion away from the satellite which is centred on Secretary Island. This local deformation signal appeared to move the ground surface away ascending satellites (track 348, frame 6260): this appears as positive motion in radians and negative motion in millimetres. In figures, the dates of the interferograms are presented in YYYYDDD, for the year and the Julian day of the acquisition. 


\subsection{Preliminary Investigation}

Preliminary investigation shows that there was localised deformation around Secretary Island at the same time as the Dusky Sound earthquake [Figure 4.1]. Figures 4.1 and 4.2 presents interferograms between acquisition before and after the Dusky Sound and Kaikōura earthquakes. We see the region surrounding Secretary Island moving away from the satellite during the period including the Dusky Sound earthquake 28 June 2009-13 August 2009 [Figure 4.1]. To a smaller degree, this signal is also present during the period 26 December 2008-10 February 2009 prior to the Dusky Sound earthquake. This localised deformation has a relative magnitude of c. 3 radians compared to the surrounding region. For period 16 September 2015-8 November 2017, we did not observe any evidence of localised deformation centred on Secretary Island, suggesting no triggered slip from a source close to Secretary Island followed the 2016 Kaiköura earthquake [Figure 4.2 and A5]. As a result of this finding, no further analysis of data from ALOS2 was completed.

As an initial approach to determine whether spatial extent and magnitude of deformation observed at Secretary Island, as seen in Figure 4.1, could be generated by a source similar to the 2003 Secretary Island earthquake we modelled the expected deformation field [Figure 4.3]. To visually match the pattern of motion away from the satellite at Secretary Island, the location of the maximum slip was horizontally shifted [Figure 4.4, left]. We then assess whether the signal of a local slip source could be resolved given the large regional deformation signal from the Dusky Sound earthquake, the deformation fields were combined. Displacement from the Dusky Sound earthquake was modelled using the parameters from Fry et al. (2010), and this was added to the forward model of a local slip source, as described above [Figure 4.4]. From this, we determined that a small expected signal from reactivated slip, in the vicinity of the 2003 Secretary Island earthquake, should be resolvable despite the relatively large surface displacement from the Dusky Sound earthquake. 

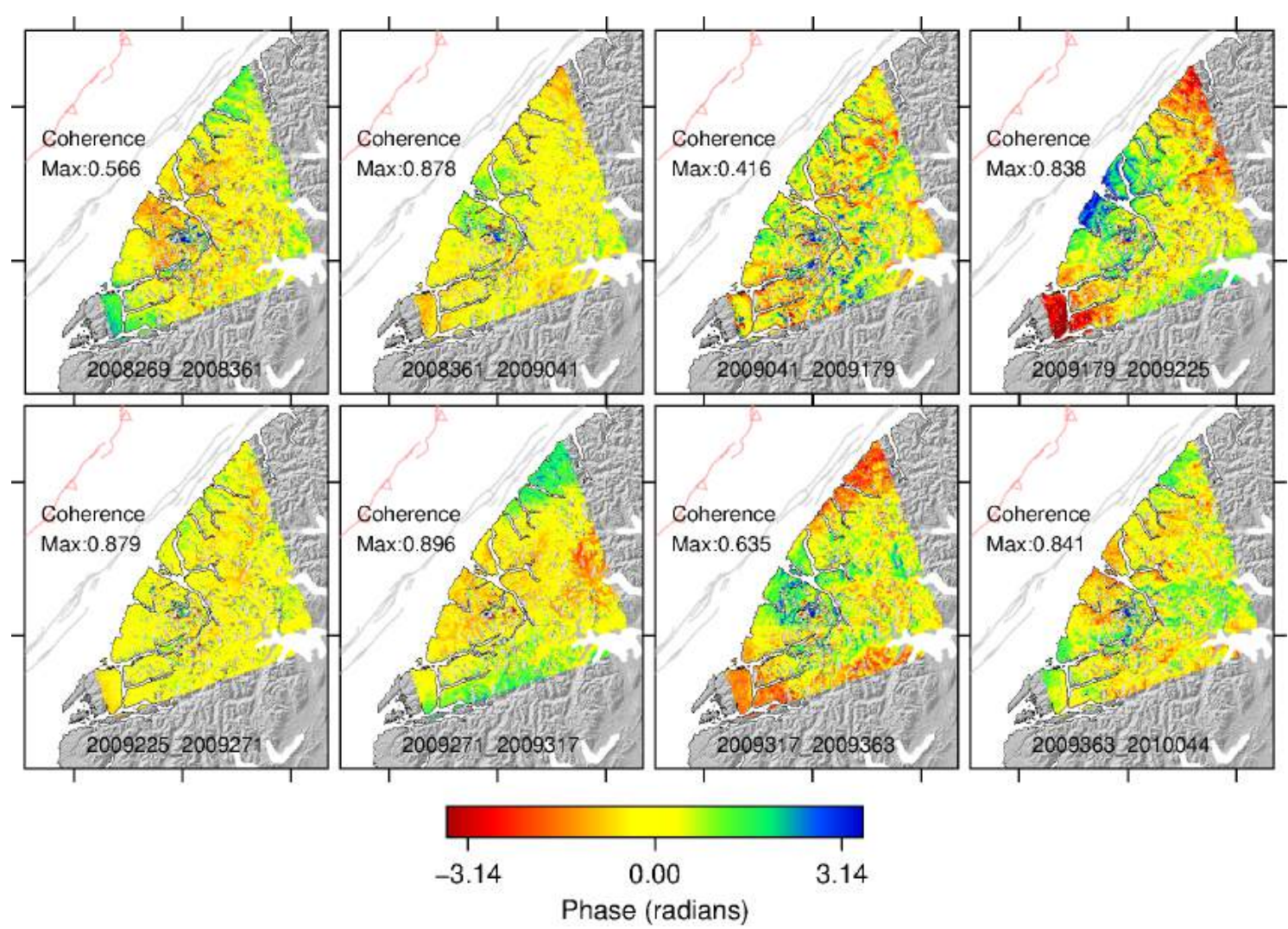

Figure 4.1: ALOS1 interferograms: track 348, frame 6260, 25 September 2008-13 February 2010. The region surrounding Secretary Island appears to move away from the satellite between 28 June 2009-13 August 2009 (2009179-2009225).
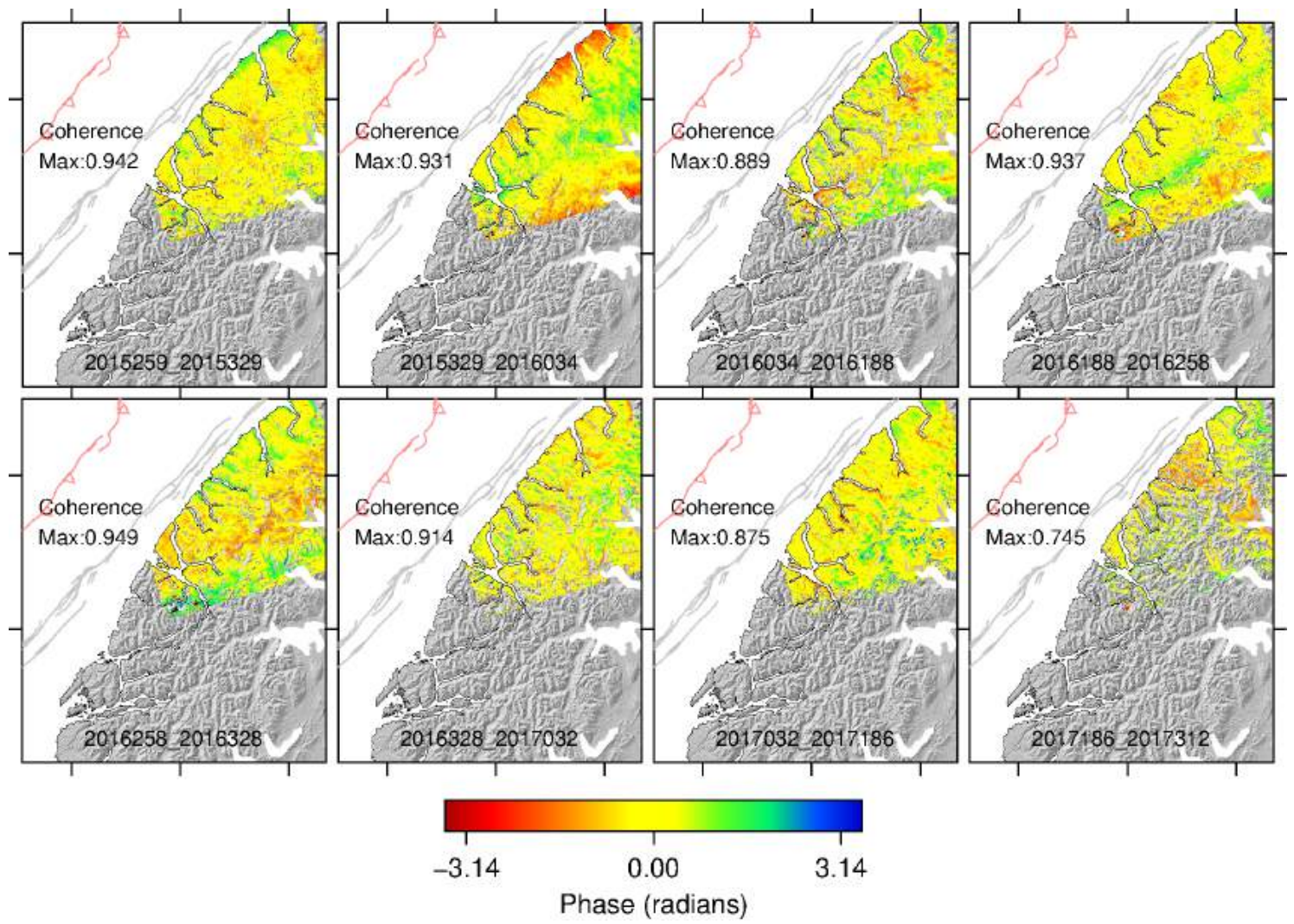

Figure 4.2: ALOS2 interferograms: track 106, frame 6270, 16 September 2015-8 November 2017. No deformation is observed during this period 


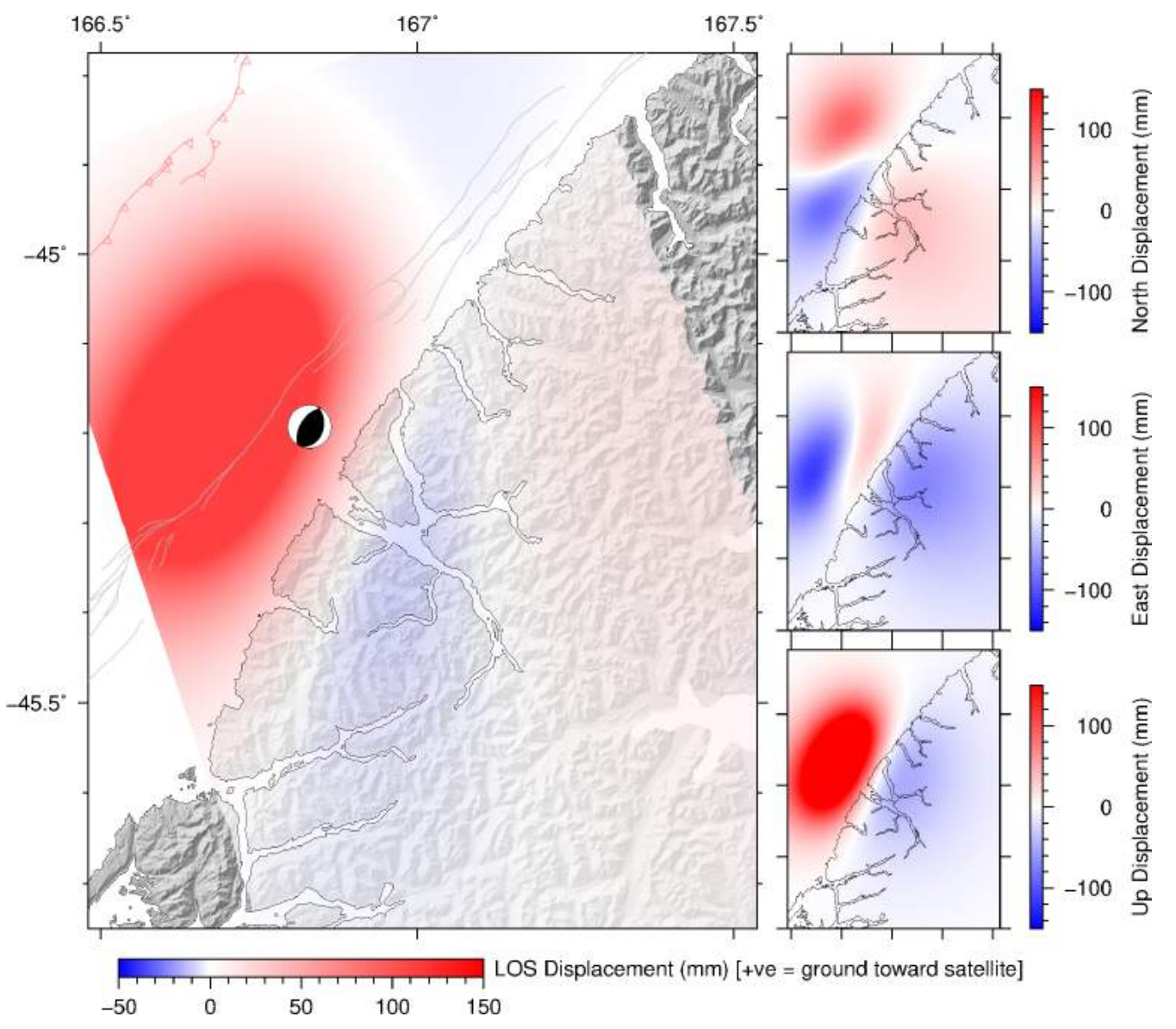

Figure 4.3: Forward model of 2003 Secretary Island displacement field based on parameters from Reyners et al. (2003); strike $030^{\circ}$, dip $30^{\circ}$, fault length and width $35 \mathrm{~km}$ and $10 \mathrm{~km}$, depth $23 \mathrm{~km}$, slip $2.4 \mathrm{~m}$ and rake $100^{\circ}$. 


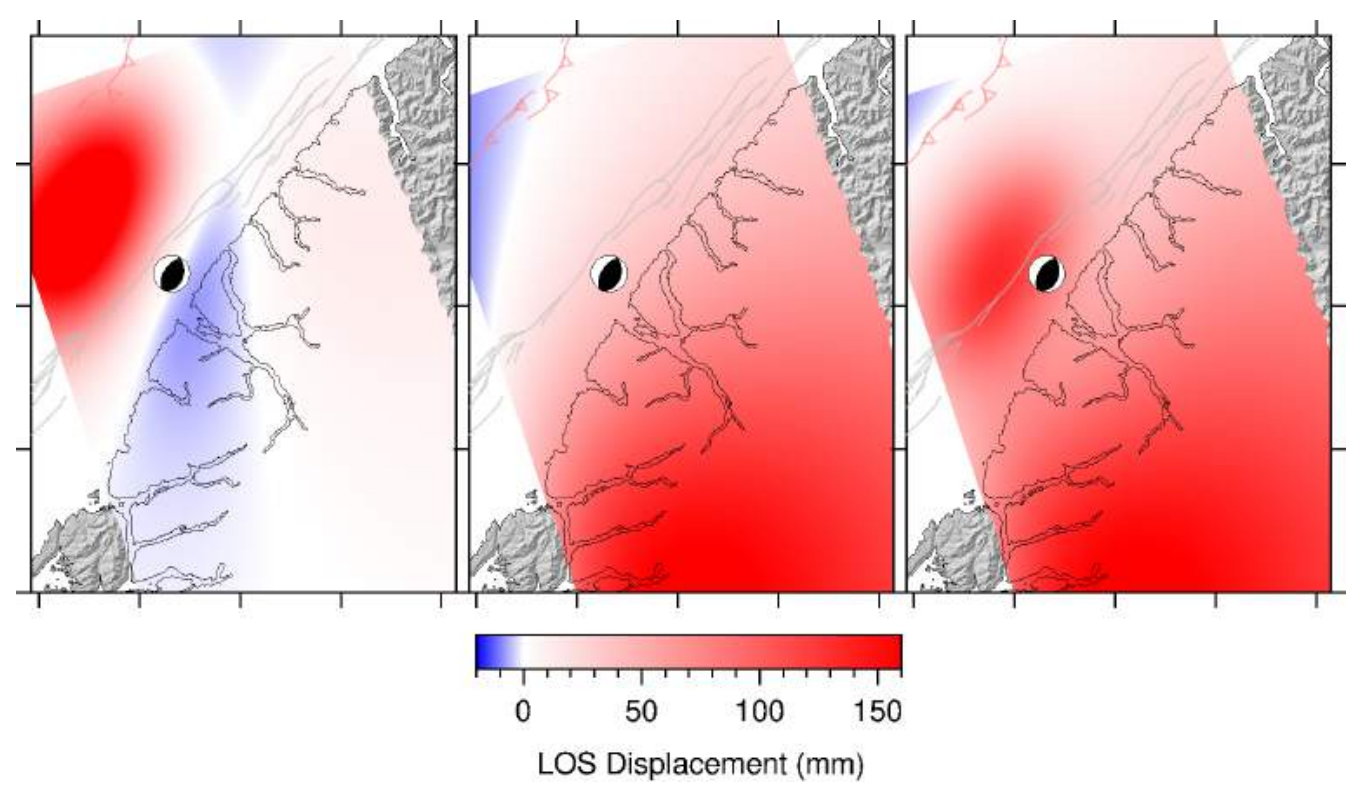

Figure 4.4: (Left) Modelled reactivated slip near Secretary Island. Fault parameters from Reyners et al. (2003) were adjusted to a maximum slip of $1 \mathrm{~m}$, and the location of maximum slip shifted $10 \mathrm{~km}$ west of the 2003 Secretary Island epicentre and the strike was increased to $35^{\circ}$. (Centre) Displacement from Dusky Sound earthquake modelled with parameters from Fry et al. (2010). (Right) The two displacements fields combined.

\section{$4.2 \quad$ Interferograms}

The final network of interferograms presented includes 96 images of varying qualities, Figure 4.5 presents a sub-sample. Interferograms with 'good' coherence had a maximum coherence of $0.8-0.9$ and median coherence of $0.4-0.5$. In contrast, 'low' coherence interferograms had a maximum coherence in the range of $0.3-0.4$ and a median coherence value of $0.04-0.1$. Coherence was not uniform across the interferograms, for images from track 348, coherence was higher along the coastline, and lower inland in the mountainous region of maximum rainfall [Figure 3.3 and A7]. These low coherence regions appear as speckled patches in the wrapped phase interferograms, despite this, some phase signal could still be extracted from these regions [Figure A8]. As shown in Figure A6, unwrapping errors were pervasive through nearly all interferograms, irrespective of coherence, prior to the application of the nearest-neighbour interpolation during unwrapping. With the application of the nearest-neighbour interpolation, interferograms with higher coherence had fewer unwrapping errors than interferograms with low coherence [e.g. Figure A17]. 

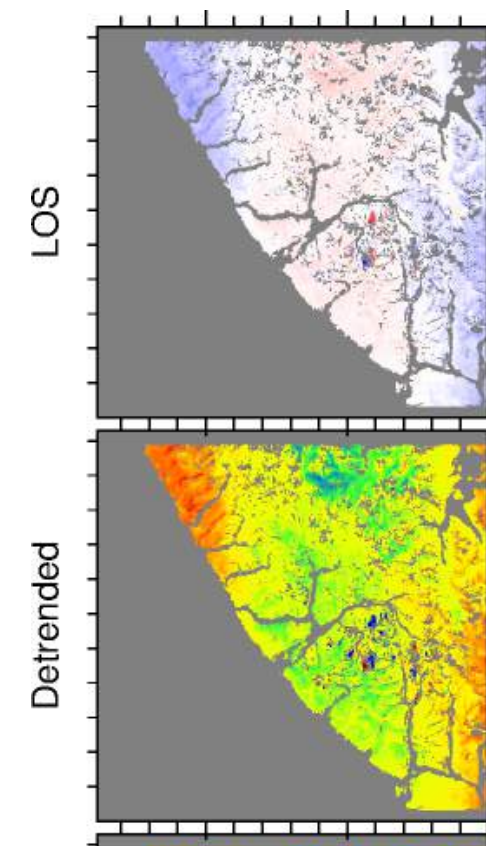

$$
\text { 高 }
$$
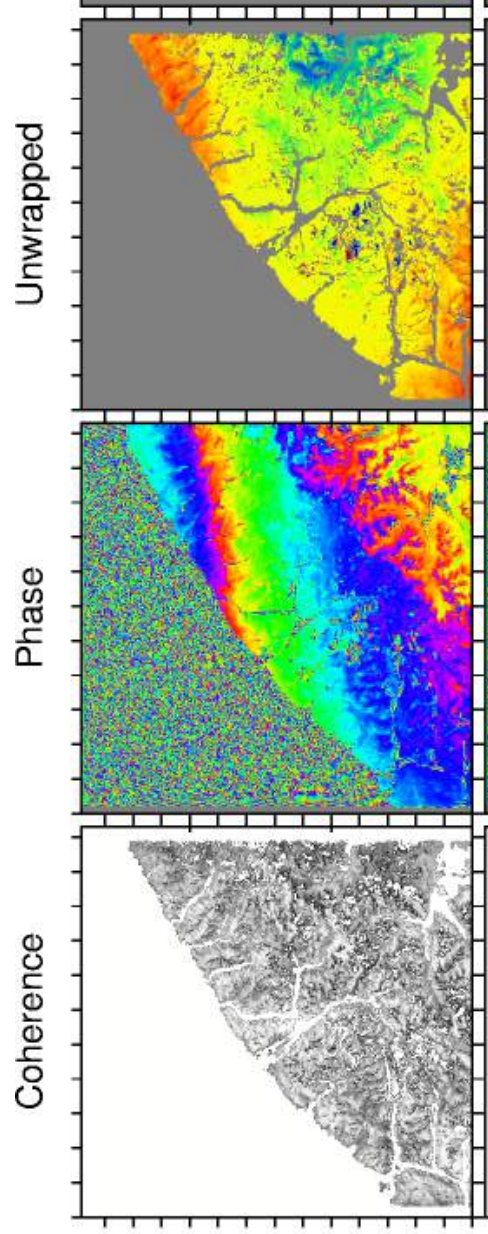

әэиәдучоว рооэ
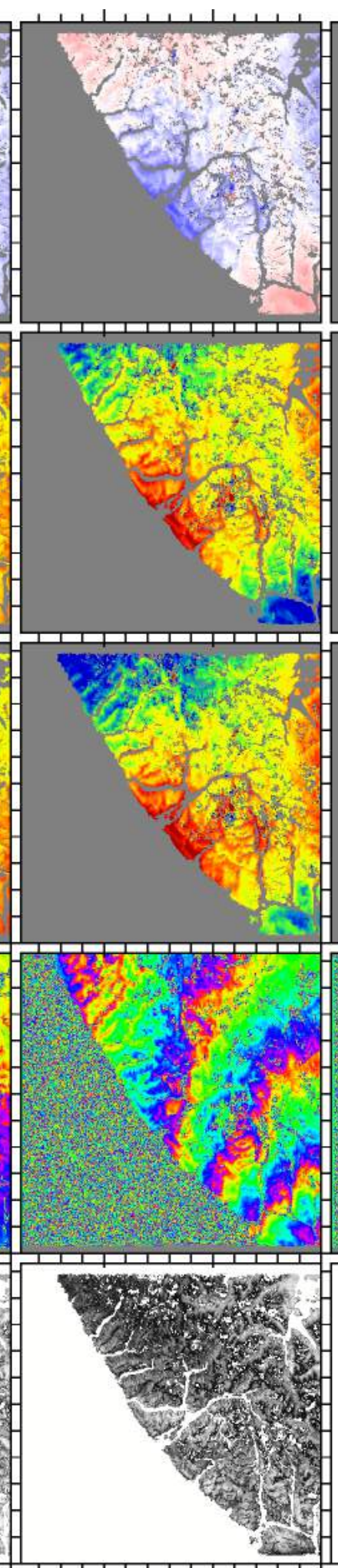

әоиәдәчоว әџедәроW
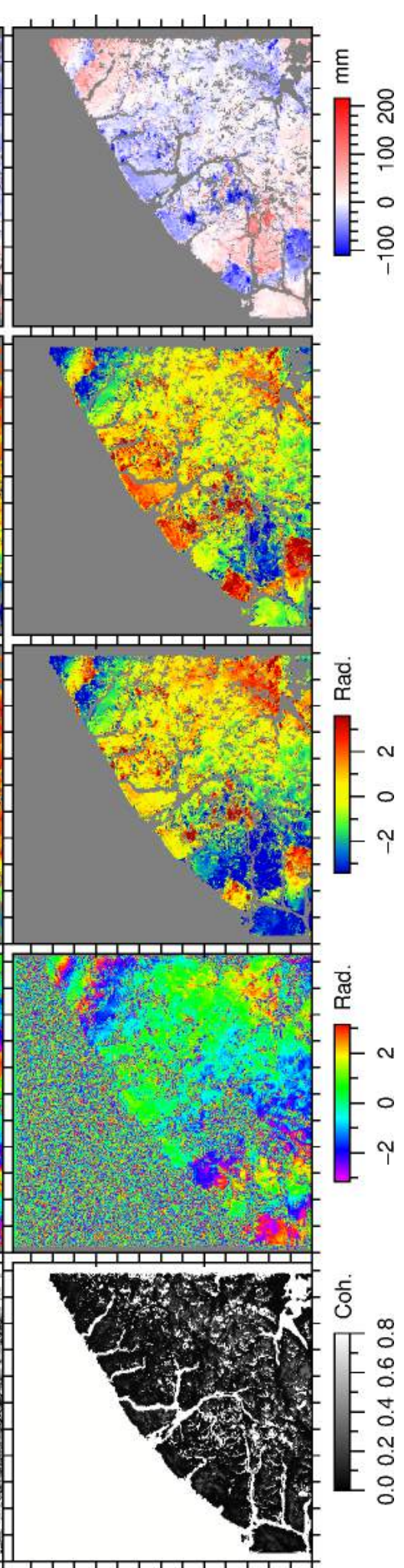

әวиวләчоว 100d
है छُ

50 皮

ช

$\overbrace{}^{-1}$

売 $\delta .0$

ช ণ

인

य

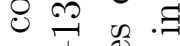

응

일

ส $\Xi$ 光

i $\infty$

$\approx 2 \infty$

t)

द्व

-

元

ठ \&

유

ही क्ये

तิ

कै

$\|$ 㻤

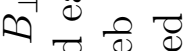

ᄋ

ํำ

웜용

乙.

m 0

거워워

ᄋ류

글

造远

次

రั

800

○ छ

$\rightarrow \infty 00$

.

ते च क

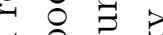

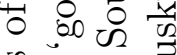

ق

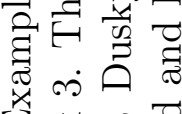

되

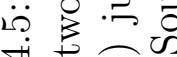

अ

$\exists$ 胥

5000 
The influence of atmosphere and ionosphere noise is clear in wrapped interferograms; tightly spaced deformation fringes appear during periods with no coinciding ground deformation [Figure 4.5, top row]. Phase gradient from ionosphere delays are often observed trending northeast-southwest [Figures A5, A8, A9 A10, A11 and A12]. Noise attributed to ionosphere delays produced phase gradients of up to 15 radians or $30 \mathrm{~mm}$ across a scene. After detrending, this was reduced to the range of 5 radians (e.g. scene 2007266-2007312 [Figure A14] and 2009179-2009271 [Figure A16]).

Figure 4.6 presents a circle test used to determine the robustness of detrending and to assess short-wavelength noise in the data. The deformation associated with the Dusky Sound earthquake and the local deformation signal near Secretary Island cancelled in the residual plot, indicating that they were accurately represented in both interferograms. The deformation from the George Sound earthquake was not captured accurately, as shown by its presence in the residual plot. This could indicate the presence of an unwrapping error, although no step in phase was identified, or error associated with removal of a linear plane. The 'speckled' noise inland correlates spatially with where coherence is typically lowest and shows that short-wavelength noise from low image coherence is still present in the final interferograms.

Figure 4.7 compares visual detections of the local deformation signal around Secretary Island detections were deemed 'unclear' if there was an unwrapping error near to Secretary Island or if the noise level was high, and the presence or absence of the signal could not be determined. The local deformation signal is most often present in pairs which span both the George Sound and Dusky Sound earthquakes. The 'unclear' detections can be categorised as pairs with long temporal separations, or pairs with short temporal baselines following the Dusky Sound earthquake. This method of bracketing the timing of potential deformation revealed a window between 28 June 2009 and 13 August 2009 that is coincident with the Dusky Sound earthquake [Figure 4.7]. Additional positive detections of interferograms with short time spans followed the Dusky Sound earthquake between 29 December 2009-13 


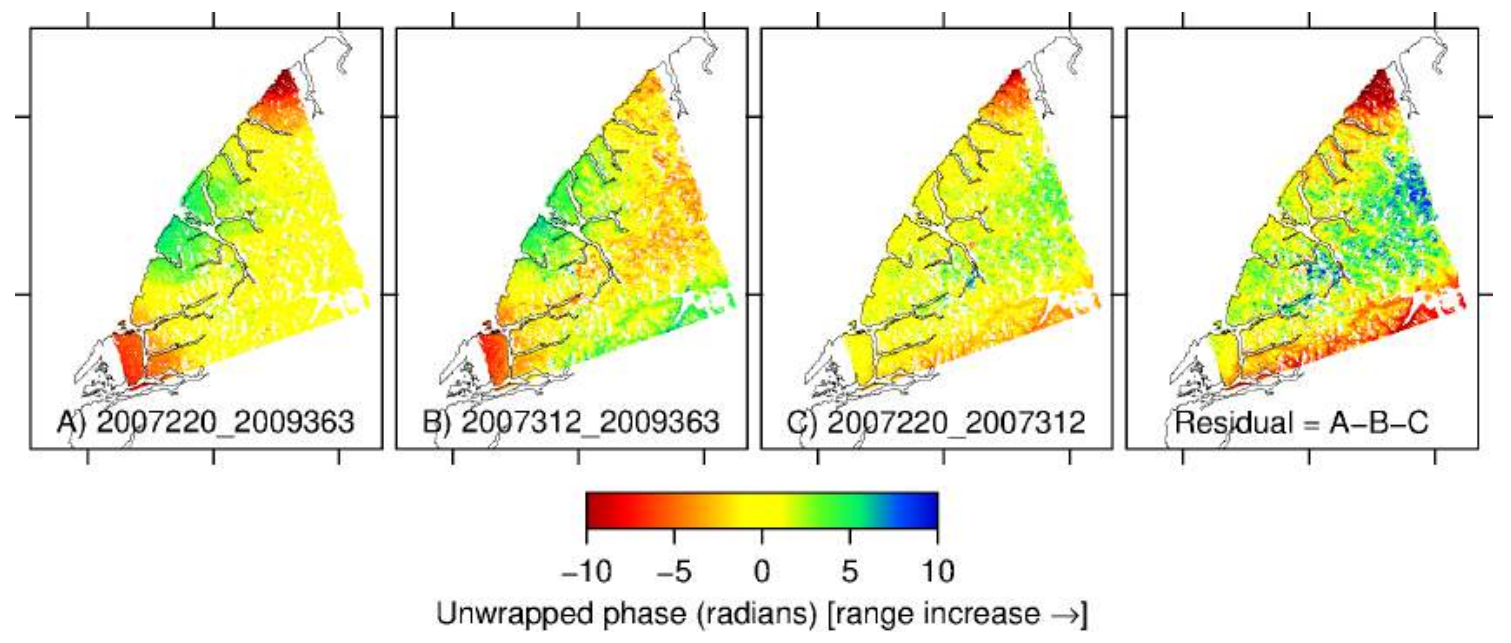

Figure 4.6: Circle test with unwrapped, detrended interferograms to determine the amount of noise within images. Interferogram A contains deformation from both the Dusky Sound and George Sound sound earthquakes, B contains only the Dusky Sound and C contains only the George Sound. The residual (A-B-C) indicates that the amount of offset from the George Sound earthquake (to the north) was under or overestimated and does not sum to zero. In contrast, interferogram A and B both captured the same magnitude of deformation for the Dusky Sound earthquake and the local deformation around Secretary Island, and these cancel in the residual plot (right). Along the south-east edge of $\mathrm{B}$ and $\mathrm{C}$ interferograms, the deformation is non-zero as would be expected, and in the residual this is compounded. Shortwavelength 'speckled' noise appears inland in interferograms B, C and the residual where coherence is lowest. 


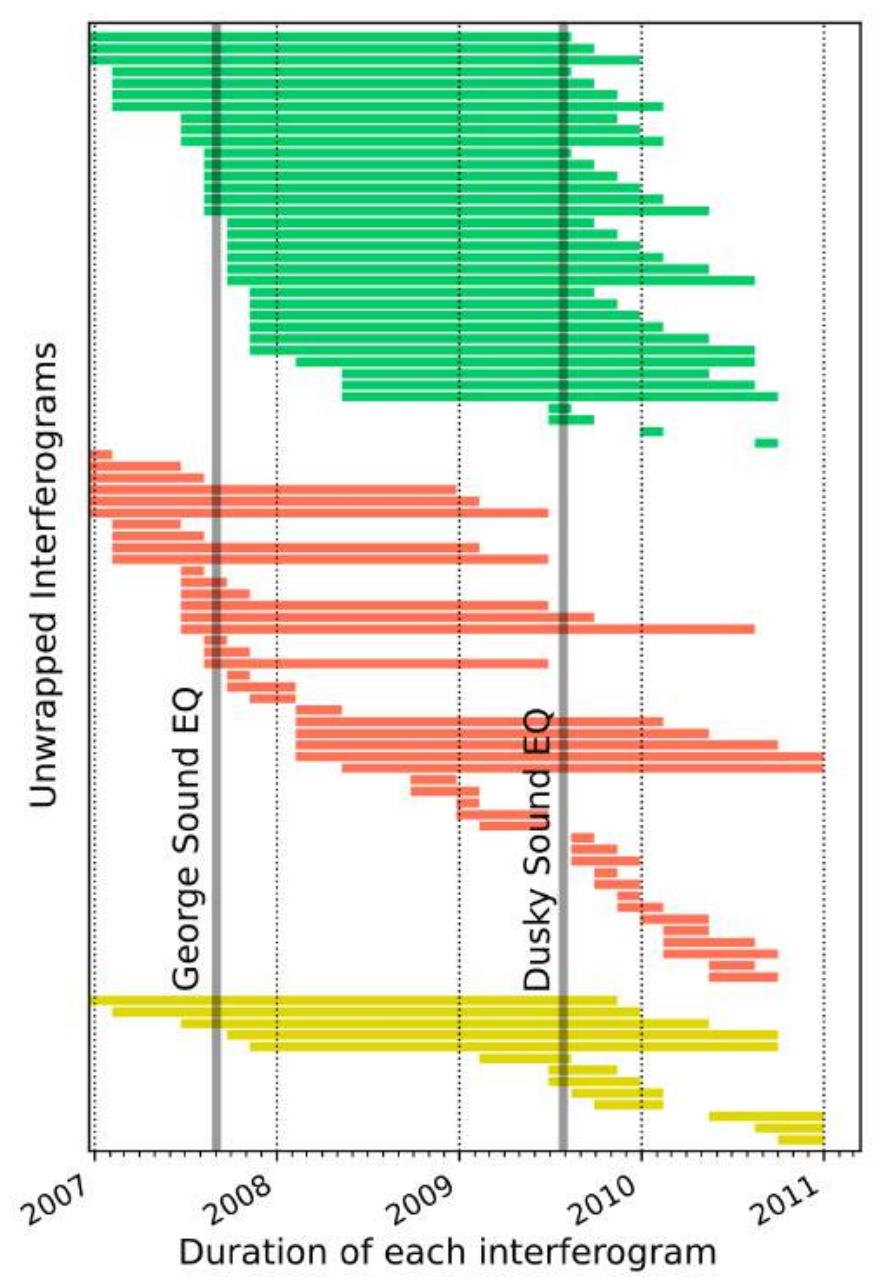

Figure 4.7: Unwrapped interferograms were visually inspected for the local deformation signal centred on Secretary Island. Green denotes signal observed, red indicates signal was not observed, yellow indicates unwrapping errors near Secretary Island or that the noise level was such the presence of signal could not be determined.

February 2010; and 16 August 2010-1 October 2010. With the full network of 96, 36 interferograms contained evidence of a region of motion away from the satellite centred around Secretary Island. The local signal was present in interferograms which spanned the periods of pre-George Sound to post-Dusky Sound, and preDusky Sound to post-Dusky Sound. The positive and unclear detections following the Dusky Sound earthquake may indicate the presence of temporally variable slip.

\subsection{Time Series}

The timeseries in Figure 4.8 presents the incremental displacement at each acquisition date for track 348, frames 6250 and 6260. From 5 February 2007 to 23 September 
2007 no change in the deformation field was detected. On 8 November 2007, the relative displacement of $227 \pm 7 \mathrm{~mm}$ towards the satellite, from the $M_{w} 6.7$ George Sound earthquake (16 October 2007) is observed (NB: maximum values in George Sound epicentral region are clipped in Figure 4.8). On the 8 November 2007, there is a slight region of relative negative LOS displacement along the north-western edge of the frame. On the 13 August 2009, displacement from the $M_{w} 7.8$ Dusky Sound earthquake (15 July 2009) is first observed. Relative maximum displacement of $104 \pm 4 \mathrm{~mm}$ towards the satellite is present the south-west region of the frame. In the centre of the frame, along the coastline, is the semi-circular region around Secretary Island (i.e. local deformation signal), which moved a maximum of $87 \pm 4$ relatively away from the satellite. The root mean square (RMS) misfit in Figure 4.9, indicates greater misfit values coeval with the regions with the largest displacements (i.e. in the north and south of the frame closer).

Cross-sections of the cumulative displacement are presented in Figure 4.9. Five profiles perpendicular from the plate boundary at $15 \mathrm{~km}$ spacing, and one $100 \mathrm{~km}$ profile parallel the plate boundary were selected. The motion prior to George Sound is presented in green, all transects were stable during this period with no motion above the background noise level observed. The period between George Sound and Dusky Sound is presented in yellow. Here we see the co-seismic offset from George Sound to the north along $\mathrm{X}-\mathrm{X}^{\prime}$ and no detectable deformation along the lines $\mathrm{A}-\mathrm{A}^{\prime}$ to $\mathrm{E}-\mathrm{E}$ '. The displacement profile along $\mathrm{X}-\mathrm{X}^{\prime}$ shows no detectable displacement to the south $(0-70 \mathrm{~km}$ along the profile), while to the north a positive linear gradient, with displacement increases to a maximum of c. $220 \mathrm{~mm}$ in the north.

Following the Dusky Sound earthquake (presented in purple) all profiles except $\mathrm{A}-\mathrm{A}$ ' show LOS displacements. $\mathrm{X}-\mathrm{X}$ ' shows the positive co-seismic offset from Dusky Sound to the south, motion away from the satellite through the centre, and positive slip towards the satellite as the profile approaches George Sound $(100 \mathrm{~km})$. Profiles B-B', C-C' and D-D' indicate increasing motion away from the satellite (negative LOS) westward along the profile, and no detectable motion 

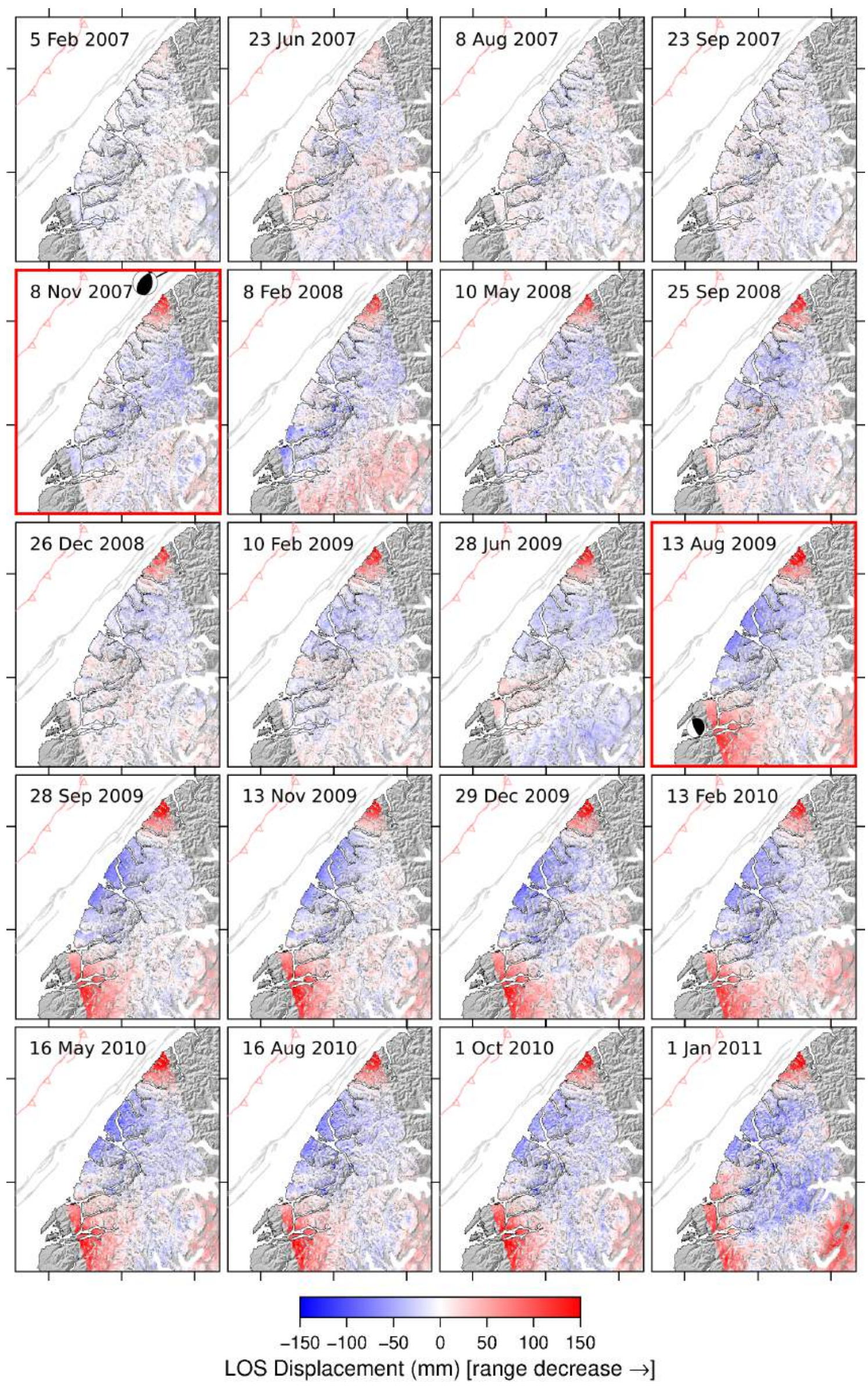

Figure 4.8: Cumulative displacement from 2007-2011. George Sound (16 October 2007) and Dusky Sound (15 July 2009) earthquakes highlighted in the red boxes. 

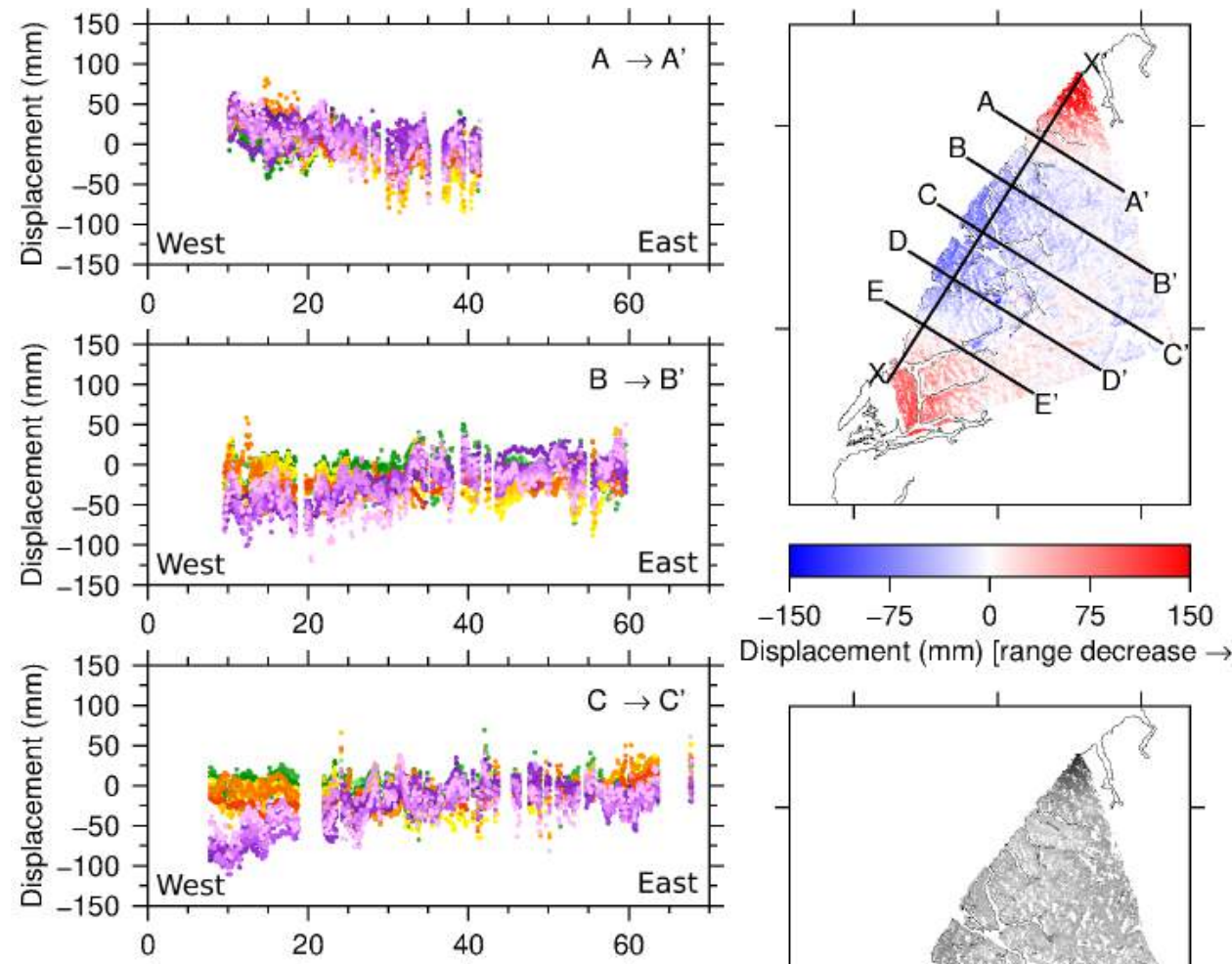

Displacement $(\mathrm{mm})$ [range decrease $\rightarrow$ ]
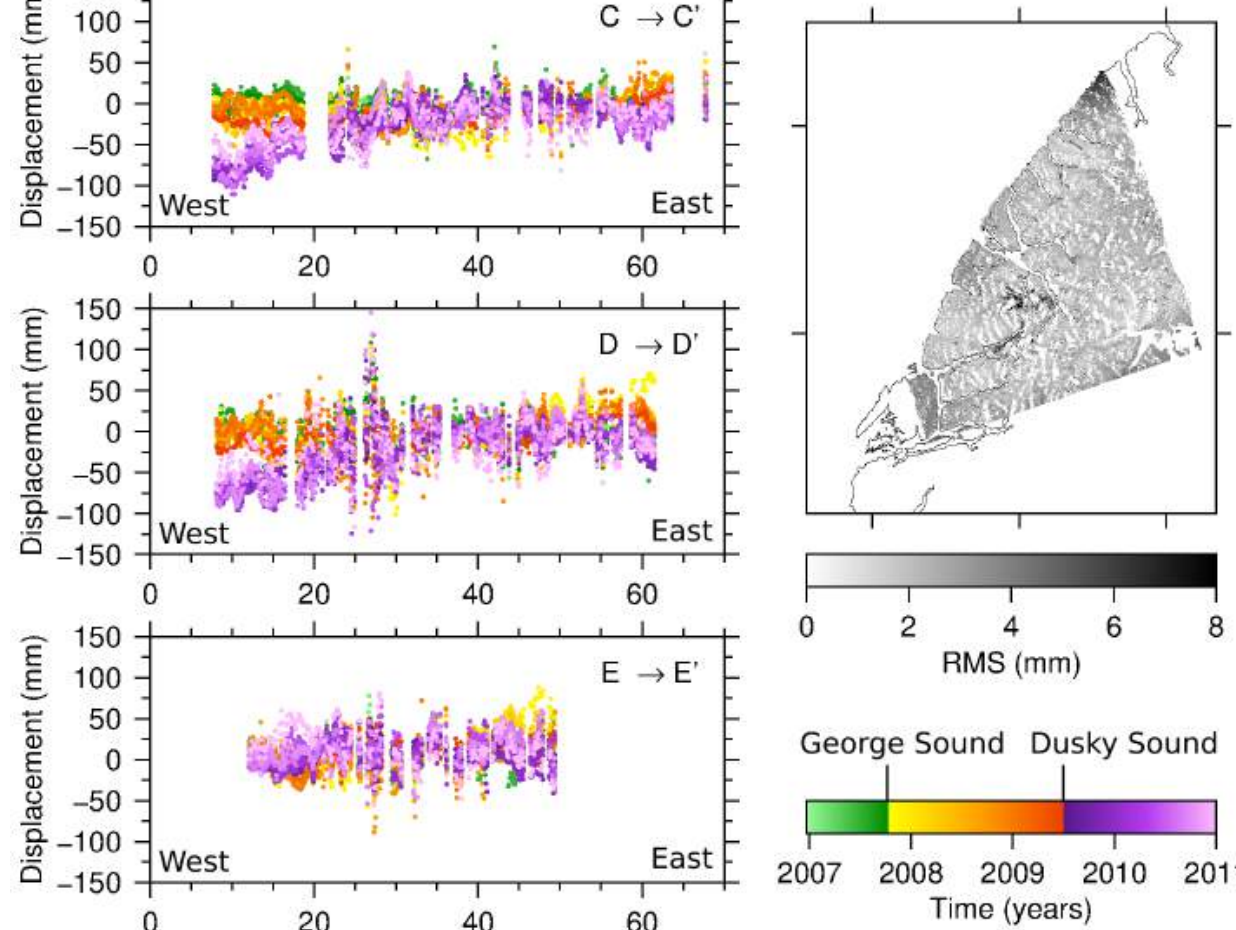

George Sound Dusky Sound
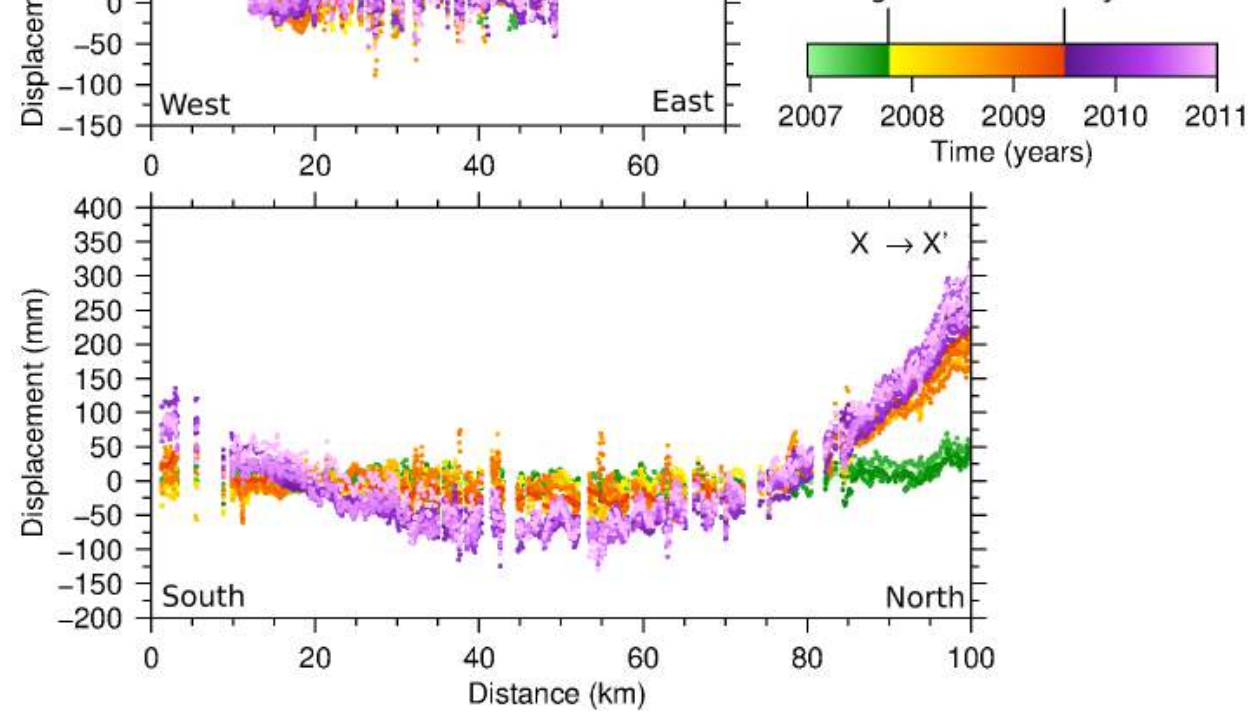

Figure 4.9: Cross section of cumulative deformation field from 2007-2011. Top subplot shows total cumulative LOS displacement at 1 Jan 2011 and the locations of cross-section lines. Bottom subplot, presents RMS values for each pixel in the timeseries inversion. 

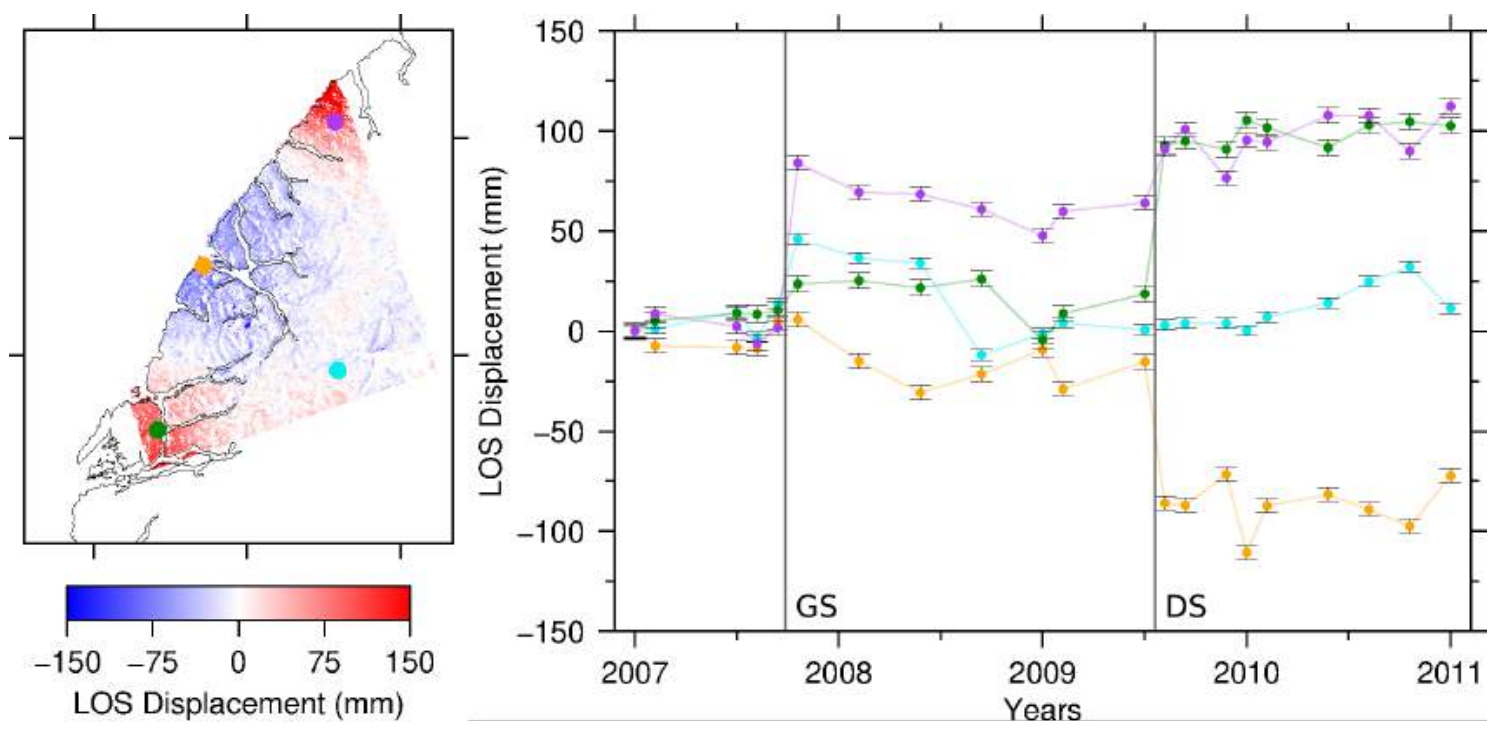

Figure 4.10: Time series showing average displacement of pixels within $1 \mathrm{~km}$ of the selected points. Green site in the southwest corner near to Dusky Sound, Orange near Secretary Island, purple is near to George Sound in the north. Blue in southeast corner which is relatively stable. Measurements are presented with the average RMS value of pixels with $1 \mathrm{~km}$ of the specified point. George Sound (GS) and Dusky Sound (DS) earthquake shown by grey lines.

inland (40-60 km along profile). This negative displacement has a wavelength on the order of ten's of kilometres: profile $\mathrm{X}-\mathrm{X}$ ' shows the downward motion has a length of $60 \mathrm{~km}$ parallel to the plate boundary, and profile D-D' shows a width of $20 \mathrm{~km}$ perpendicular to the plate boundary. The additional positive displacement after the Dusky Sound earthquake in the north is in agreement with reactivated slip at George Sound reported by Hamling and Hreinsdóttir (2016).

Figure 4.10 presents the relative cumulative displacement of selected pixels located in the north, south, near Secretary Island, and on a relatively stable location inland from Secretary Island. The LOS offset from both the George Sound and Dusky Sound earthquakes clear as steps in the timeseries. Before the George Sound earthquake, all points are within c. $25 \mathrm{~mm}$ of each other, this is indicative of the scatter in the data. The pixel near Secretary Island shows a negative LOS offset of c. $80 \mathrm{~mm}$ following the Dusky Sound earthquake, with outlying values on 29 December $2009(-117 \mathrm{~mm})$ and 1 October $2010(-96 \mathrm{~mm})$. Following the Dusky Sound earthquake, the pixel located near Secretary Island almost mirrors the pixel in the south near Dusky Sound, with an equal but opposite magnitude of relative 
displacement. After the Dusky Sound earthquake, the pixels in the north and south of the frame are again within c. $25 \mathrm{~mm}$ of each other. The variability of the pixel on Secretary Island after the Dusky Sound earthquake is larger than other pixels, this variability may reflect slip from a temporally variable local source.

\subsection{Dusky Sound Modelling}

The co-seismic offset from Dusky Sound was measured using ascending Synthetic Aperture Radar (SAR) images for the periods 12 January 2009-15 July 2009 and 6 June 2009-13 August 2009, and descending for the period 20 July 2008-23 July 2009. Figure 4.11 presents the wrapped, unwrapped, and LOS displacement interferograms. These interferograms were not detrended, as a linear plane and static offset were solved for during modelling. Interferograms along tracks 348 and 349 had moderate coherence, while interferograms along track 639 had relatively good coherence. The temporal and baseline separations were 184 days and $1037 \mathrm{~m}$ for track 349, 363 days and $342 \mathrm{~m}$ for track 639, and 46 days and $370 \mathrm{~m}$ for track 348 . Track 349 had unwrapping errors which were manually corrected for in the north of the frame where fjords and data gaps isolated the data and phase could not be continuously unwrapped.

Track 349 had a maximum LOS displacement of $682 \mathrm{~mm}$ towards the satellite located south-east of the Dusky Sound epicentre. Track 639 measured $741 \mathrm{~mm}$ LOS displacement away from the satellite, with the maximum displacement coeval with the location of the Dusky Sound epicentre. This $60 \mathrm{~mm}$ difference in LOS displacement can be accounted for, in part, by post-seismic deformation; continuous Global Positioning System (GPS) at Puysegur Point (PYGR) showed post-seismic deformation of c. $19 \mathrm{~mm}$ west and c. $30 \mathrm{~mm}$ south in the first week following the earthquake (Beavan et al., 2010). As PYGR is located c. $50 \mathrm{~km}$ away from the epicentre, we expect these values to underestimate the amount of after-slip in the epicentral region. The spatial deformation patterns for each track were different, with track 639 showing concentric fringes of phase change centred around the Dusky Sound epi- 


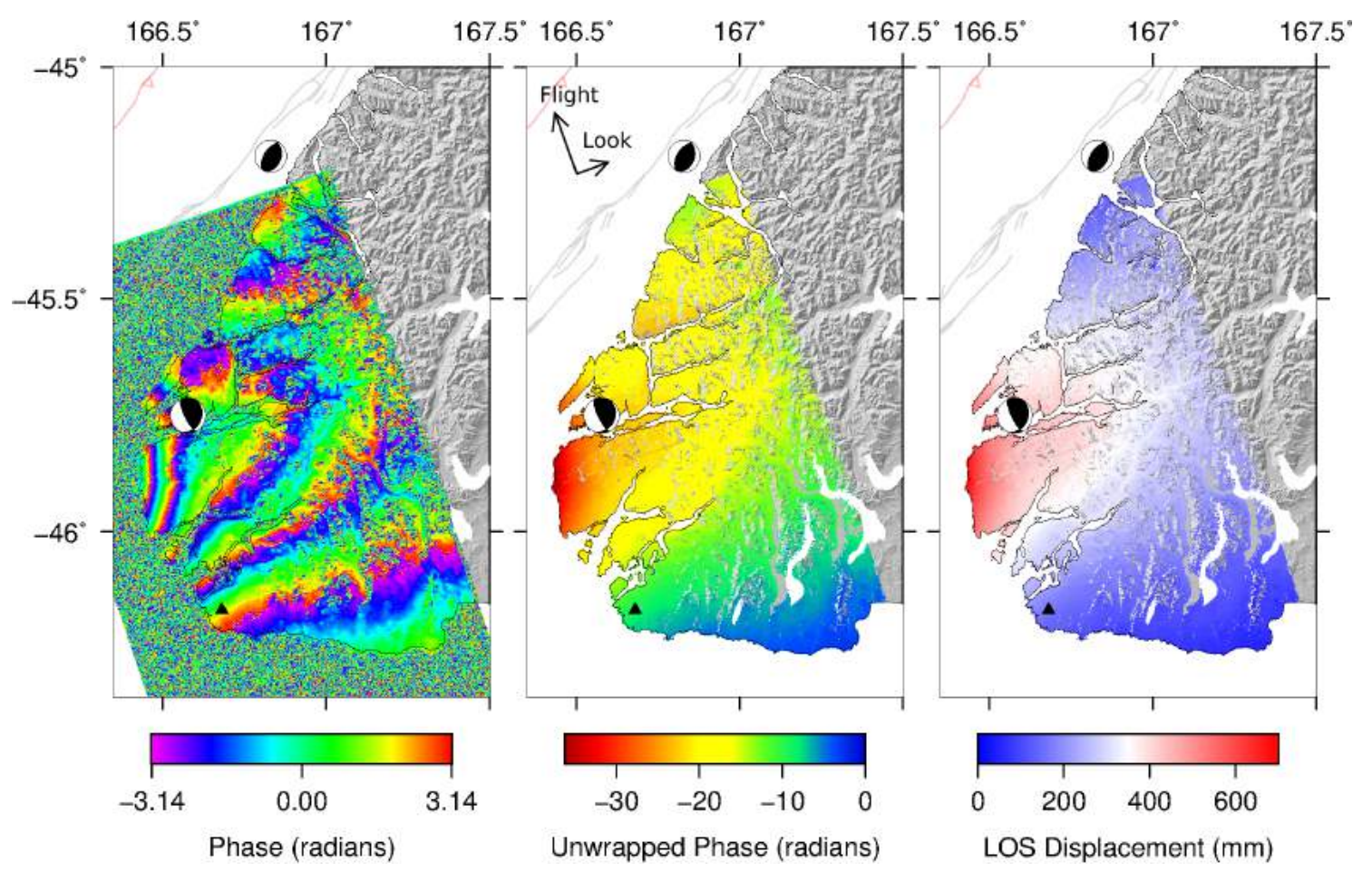

(a) 12 Jan 2009-15 Jul 2009, Track 349, Frames 6240 and 6250

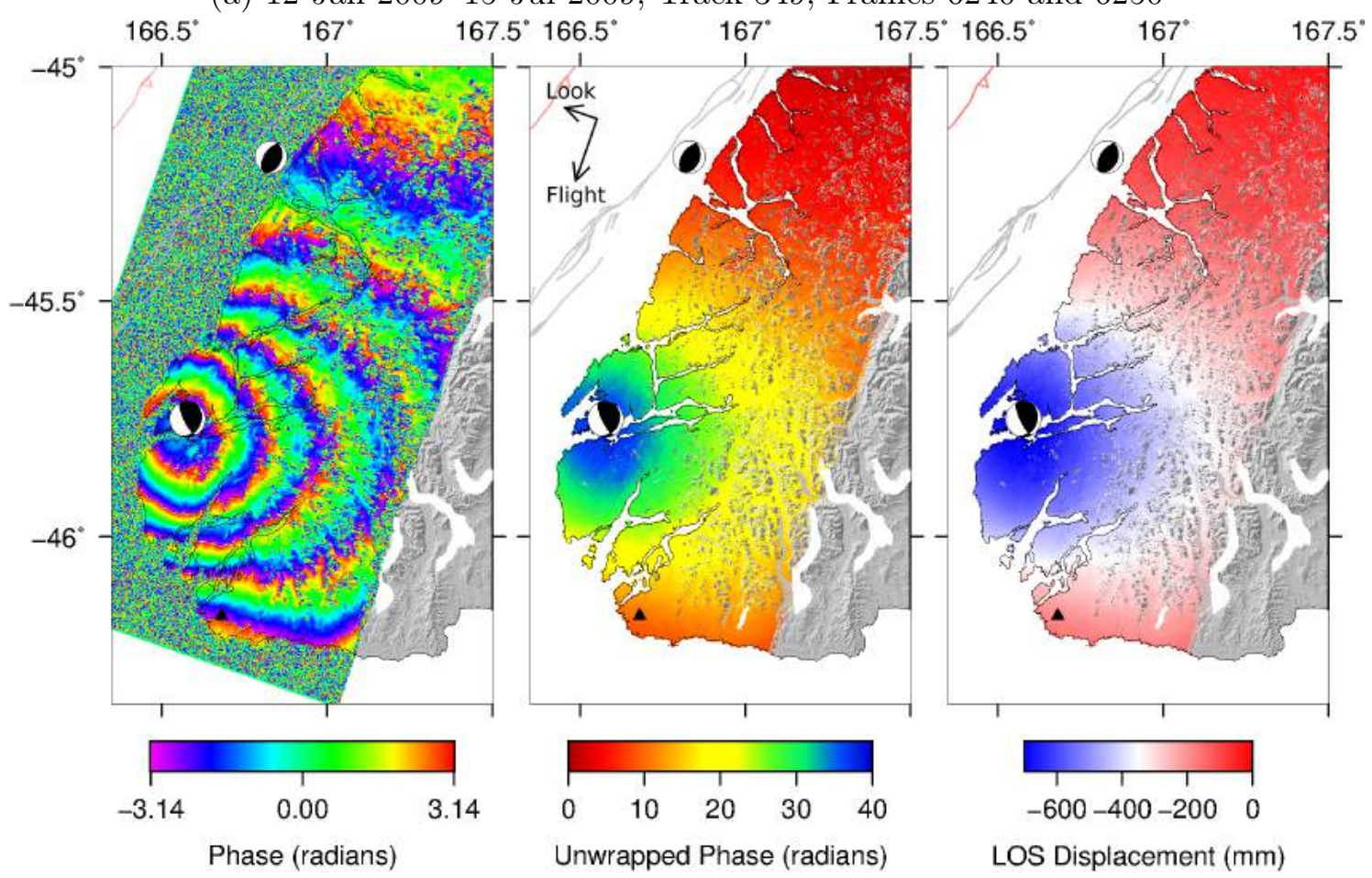

(b) 20 Jul 2008-23 Jul 2009, Track 639, Frames 4530, 4540 and 4550 


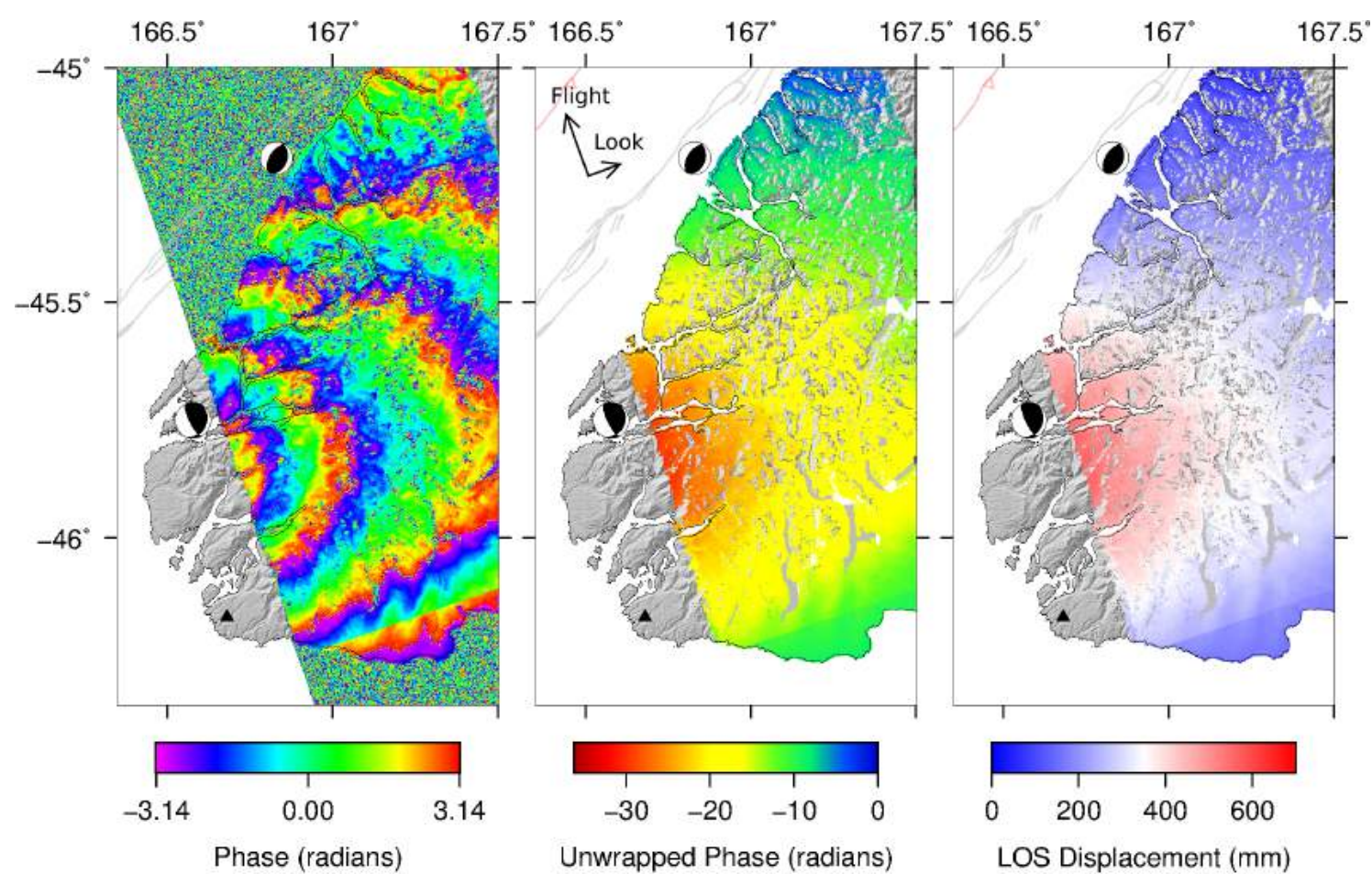

(c) 6 Jun 2009-13 Aug 2009, Track 348, Frames 6240, 6250 and 6260

Figure 4.11: Interferogram of the Dusky Sound earthquake used for modelling. Filtered wrapped phase (left), unwrapped phase (middle) and LOS displacement (right). Focal mechanism indicate location of 2003 Secretary Island and 2009 Dusky Sound earthquakes.

centre, and tracks 348 and 349 showing a broader asymmetric pattern, with fringes of phase change forming $U$ shapes away from the epicentre. The differences in the deformation patterns are likely due to long-wavelength ionosphere noise and the different observation geometries.

Table 4.1 presents the results test inversions for each individual track with GPS, and the final inversion with interferograms from tracks 349 and 639 and GPS data. Earlier inversions were completed using only InSAR data, however, these models did not converge. Although spatially sparse and acquired after the Dusky Sound earthquake, the GPS data provided bounding values for the InSAR, which stabilised the inversion, and allowed the fault parameters to converge during the inversion. The GPS data constrained the far-field deformation beyond the InSAR coverage and provided absolute measurements for the near-field deformation. 


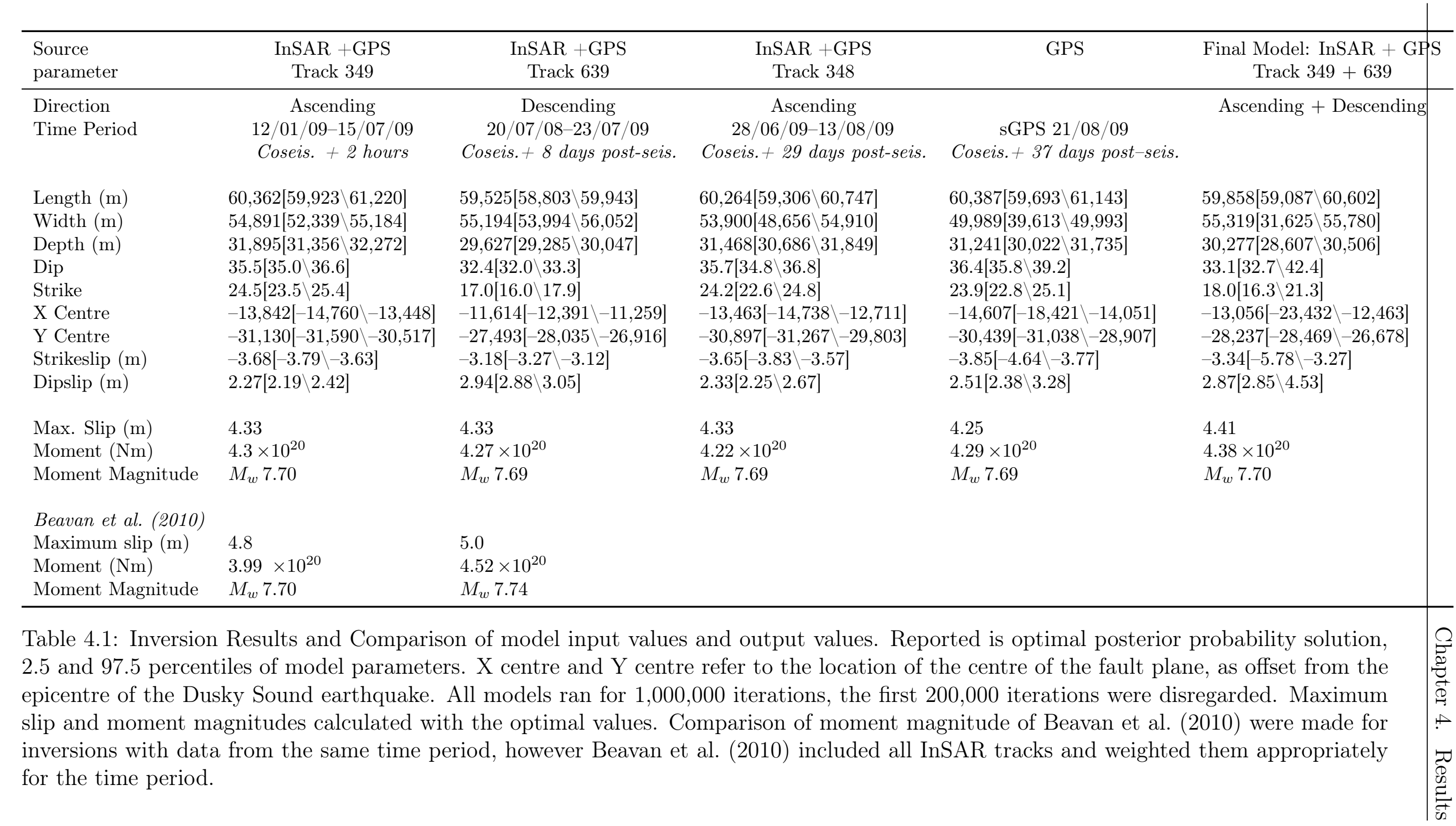


The optimal values for fault length across all tests were within $1 \mathrm{~km}$ of each other and within the 2.5 and 97.5 percentiles of the final model. Optimal values for fault width varied by $6 \mathrm{~km}$, depth by $2 \mathrm{~km}$ and dip by $3^{\circ}$. The ascending interferograms and the GPS had a strike of c. $24^{\circ}$, while the descending track had a strike of $17^{\circ}$. All tests placed the centre of the fault $29-33 \mathrm{~km}$ southwest of the epicentre. The magnitude of slip for all InSAR and GPS inversions was $4.33 \mathrm{~m}$ with the contributions of strikeslip and dipslip motion varying slightly. The GPS only inversion had slightly less slip of $4.25 \mathrm{~m}$. As the fault geometries and amount of slip were similar between the tests, the moment magnitude was only varied by 0.01 . The result that each of the tests produced the same amount of slip was unexpected as GPS from Beavan et al. (2010) indicated post-seismic deformation, and there was a difference in the GPS aligned interferograms, as presented above. We suggest that the results from tests were similar due to the high number of model iterations and that a linear plane and static offset was simultaneously solved for.

As the modelling of the Dusky Sound earthquake was for the purpose of removing the co-seismic signal and observing the post-seismic signal, the final model was generated by simultaneously inverting tracks 349, 639 and the GPS data. One million iterations of the model were performed and the fault parameters converge after 200,000 iterations [Figure 4.12a]. probability density functions (PDFs)'s were calculated from model results after the fault parameters began to converge [Figure 4.12b]. All parameters had uni-model distributions, with fault width slightly skewed to the lower values, and strike-slip and dip-slip skewed to the higher values. The joint probabilities were compared to identify relationships between fault parameters in the inversion [Figure 4.13]. The final model produced a fault with length and width of $60 \times 55 \mathrm{~km}$, and depth to the bottom edge of the fault at $30 \mathrm{~km}$. The fault strikes $18^{\circ}$ and dips $33^{\circ}$ to the southeast, with the centre of the fault located $29 \mathrm{~km}$ southwest of the Dusky Sound epicentre. The modelled fault had a uniform slip of $4.4 \mathrm{~m}$, producing a moment magnitude of $M_{w} 7.7$.

The observed, modelled and residual deformation fields are presented in Figure 4.14. The model fits track 349 better in the near-field and track 639 better 
in the far-field. Track 639 has misfit of greater than $0.1 \mathrm{~m}$ in the near-field, while track 349 has a similar magnitude of misfit in the southern portion of the track (frame 6240) [Figure 4.14]. The horizontal displacements from GPS have a similar misfit as track 639, with the modelled and observed values diverging with increasing proximity to the Dusky Sound epicentre [Figure 4.15]. The lack of near-field fit is attributed to an oversimplification of the source geometry of the earthquake. The residual from track 349 also has topographically correlated residuals. This is a result of a lower coherence threshold being applied so noisy pixels were not masked before the removal of the model. These results will be compared with those of Beavan et al. (2010) in Chapter 5.

\subsection{Isolating Local Deformation Source}

To isolate the local deformation signal centred on Secretary Island, the Dusky Sound model was subtracted from the observations, and the residual data were detrended [Figure 4.16]. To determine whether the rupture patch of the 2003 Secretary Island earthquake was reactivated by the 2009 Dusky Sound earthquake, or a similar source nearby, local source models were fitted to the residual local deformation field. The results were then examined to see if removal of a local source model improved the solution, and what the threshold of resolvable slip is given the noise level of the data. Figure 4.16 provides a summary of each step in isolating and modelling the location deformation signal.

As Figure 4.7 indicated, the temporal window for reactivated slip coincided with the Dusky Sound earthquake, hence we use the interferogram spanning the period 28 June 2009-13 August 2009, from track 348, frame 6260, to isolate the local deformation source. The interferogram was tied to the GPS co-seismic offset value of site DF4L located southwest of Secretary Island [Figure 4.16, top left]. The Dusky Sound model, as described above, was subtracted from the data producing 'Residual 1' (top row of Figure 4.16). The Dusky Sound model has a clear northsouth, deformation gradient and when detrended the residual has a strong south- 

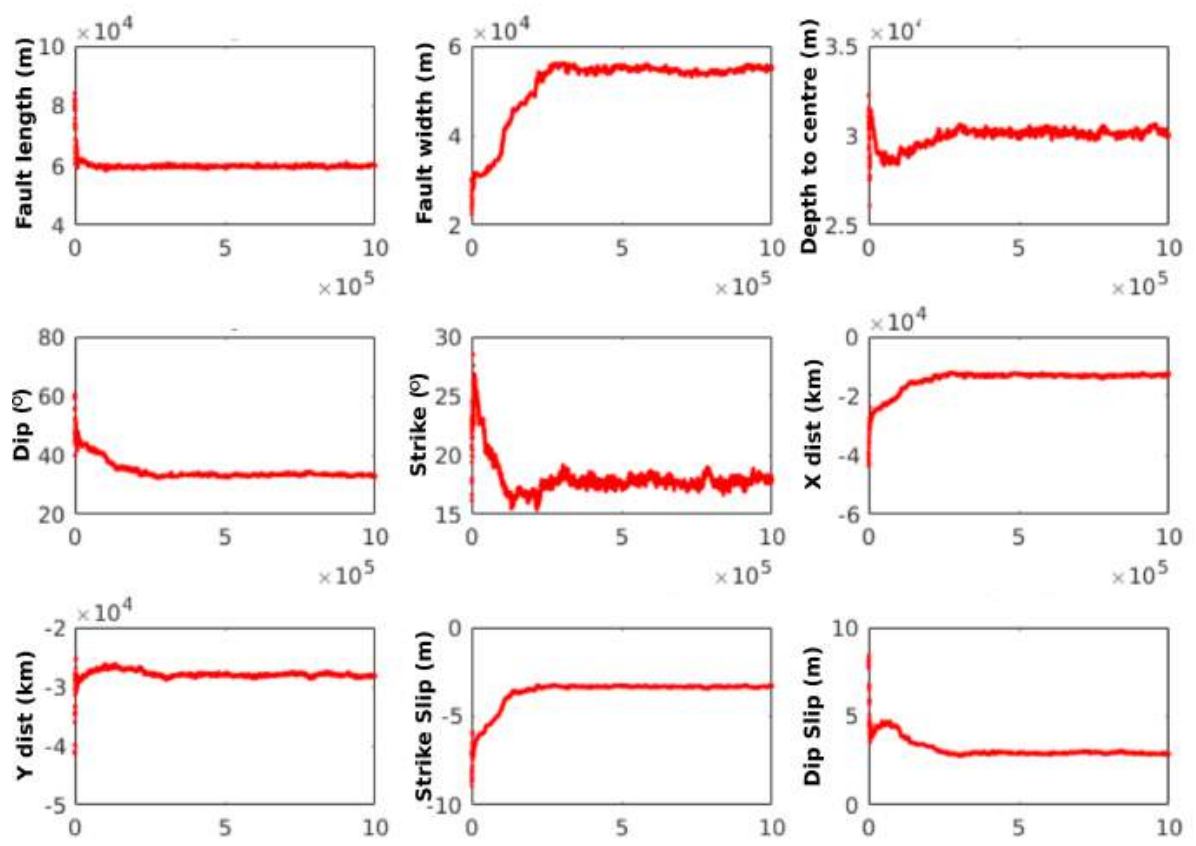

(a) Convergence plots for each model parameter
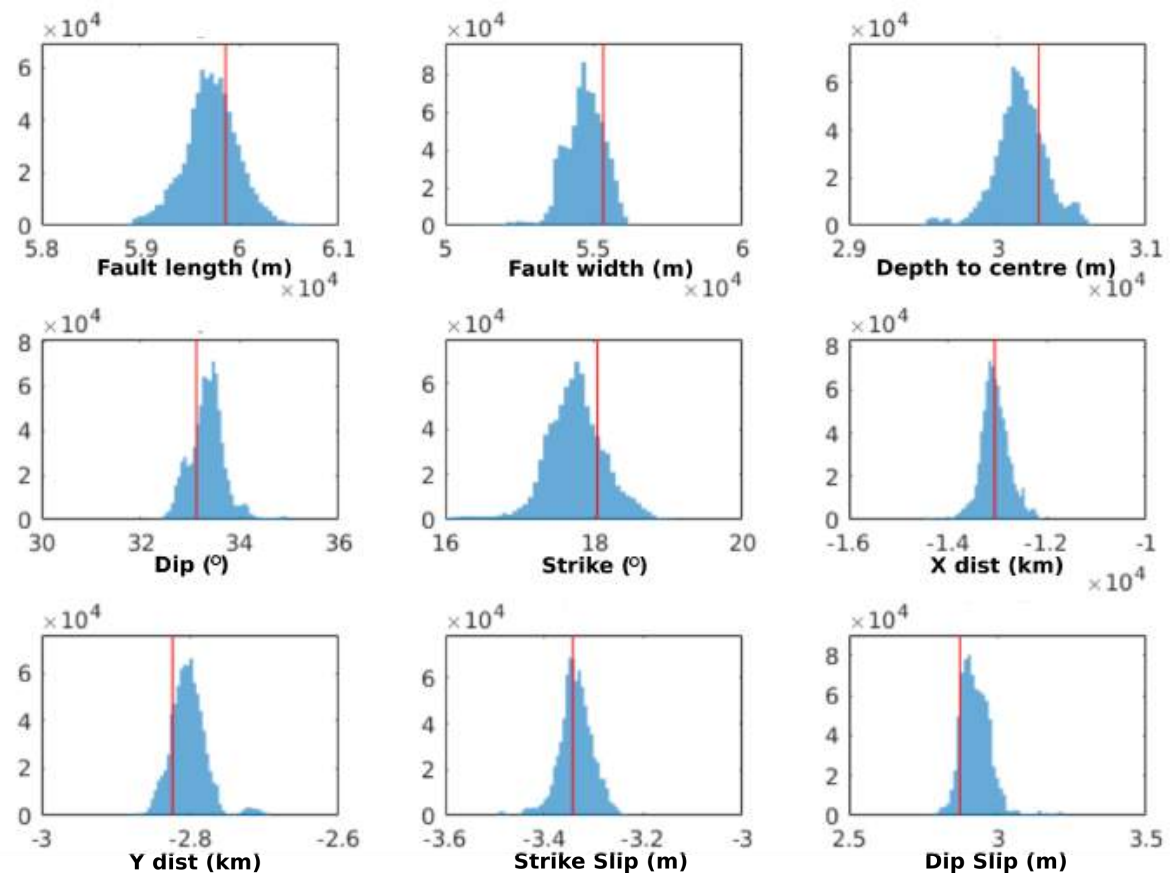

(b) Posterior probability distribution functions for final model

Figure 4.12: Convergence and PDF plots for final model parameters. For both plot, frequency is on the $\mathrm{x}$-axis and the fault parameter is on the y-axis. Red lines in (b) represent the maximum a posteriori probability solution. 


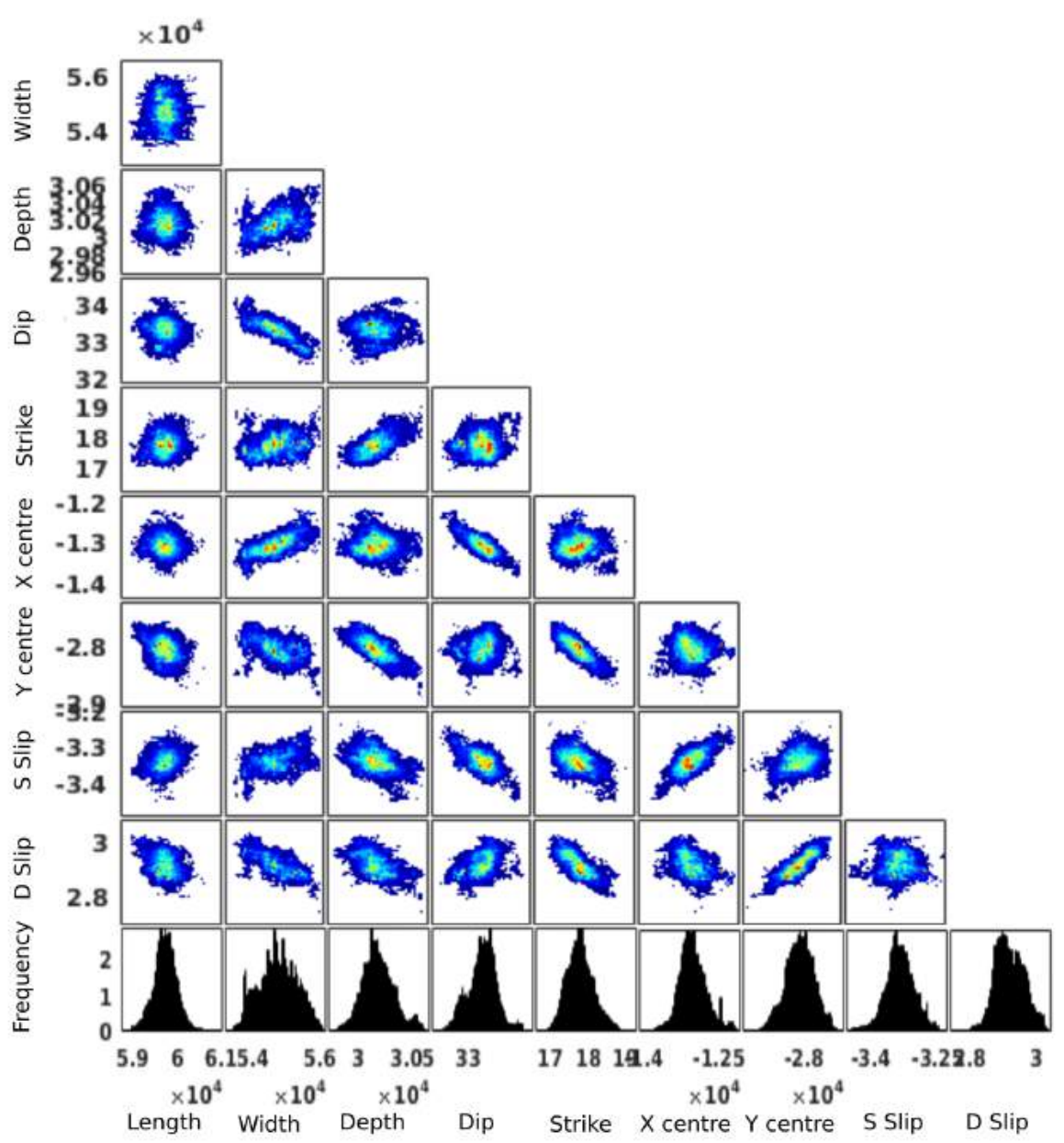

Figure 4.13: Joint Probabilities for Dusky Sound earthquake. Final model with InSAR (tracks 349 and 639) and GPS. This plot highlights the parameters which are not independent of each other. As fault width increasing the dip becomes shallower, as the depth increasing the strike increases and the fault centre moves south-west. As the fault centre moved west the amount of strikeslip increased, and as the fault centre moved south the amount of dipslip increased. 
DATA
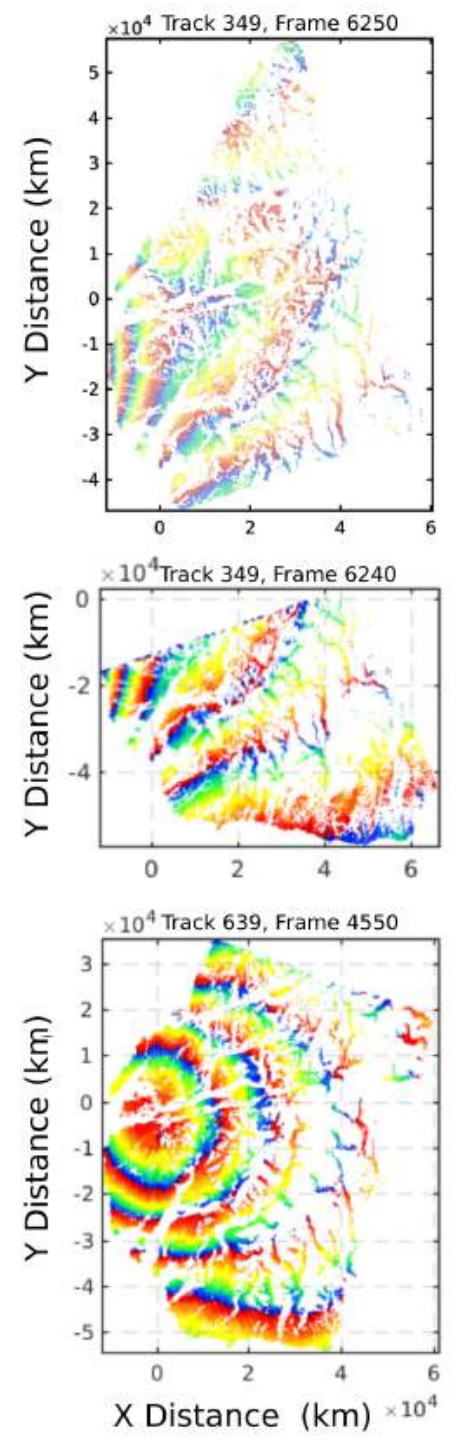

MODEL
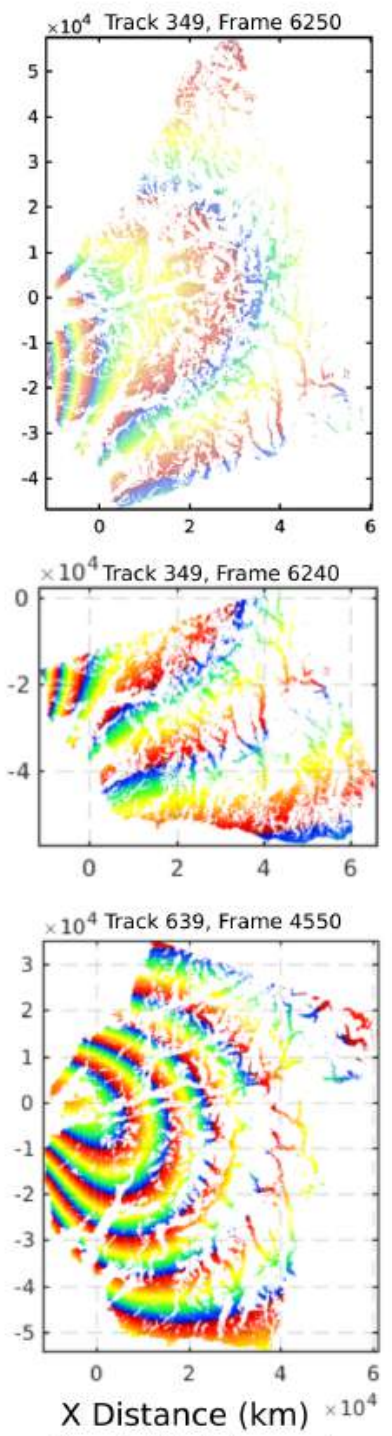

Line-of-sight displacement $\mathrm{m}$ $\begin{array}{lll}0 & 0.05 & 0.1\end{array}$
RESIDUAL
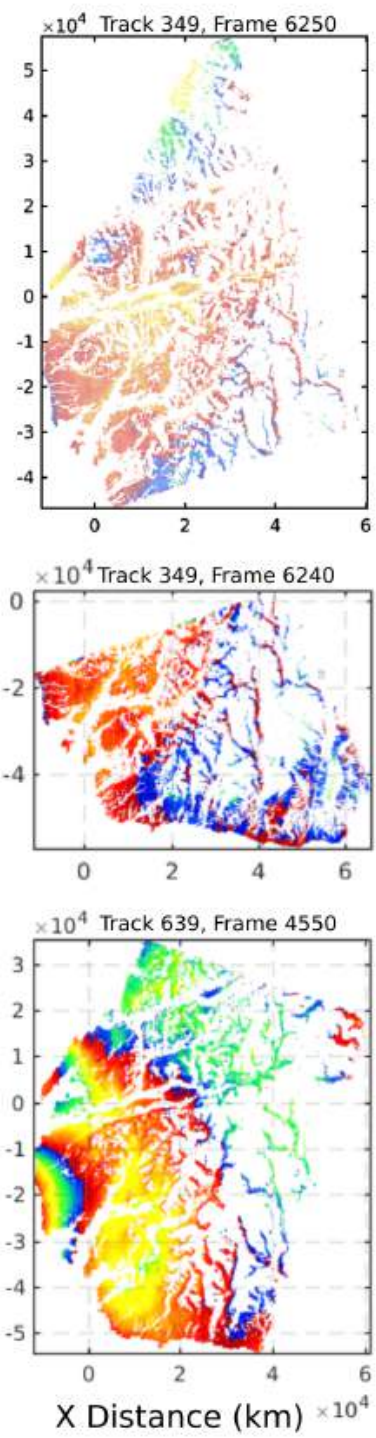

Figure 4.14: Comparison of observed, modelled and residual data for the uniform slip model of the 2009 Dusky Sound earthquake. The near-field deformation in track 639 (bottom) is not well fitted, with greater than $0.1 \mathrm{~m}$ of misfit west of the Dusky Sound epicentre. Track 348 (top and middle) is fitted well in the near-field but data in the north and south of the track do not fit as well. Figures are plotted relative to a local origin at the Dusky Sound epicentre. 


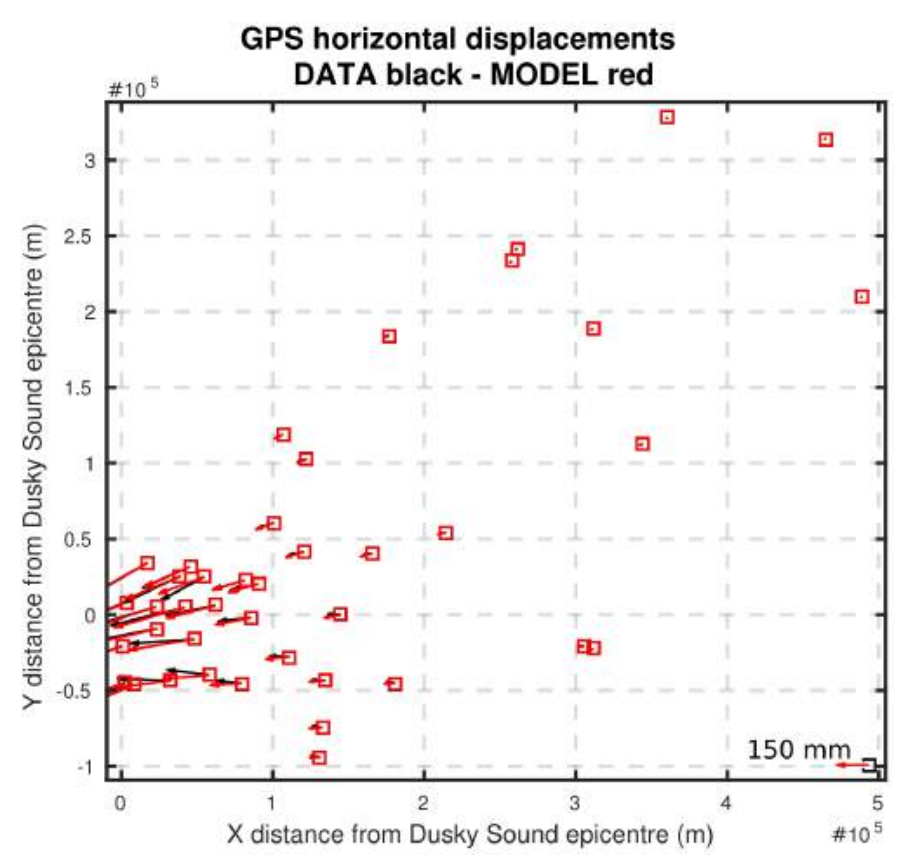

Figure 4.15: Data and Model of GPS horizontal displacement. Similar to the results of the InSAR derived deformation field, the modelled horizontal velocity do not fit as well in the epicentral region.

north gradient (Residual 1).

The trend of Residual 1 was calculated and removed, producing 'Residual 2' (middle row of Figure 4.16). A correction was also made to remove topographically correlated noise to reduce the scatter in the data. The deformation pattern of Residual 2 includes positive LOS displacement (towards the satellite) in the south, indicating not all deformation associated with the Dusky Sound earthquake was removed. As the interferogram was only tied to an absolute reference frame with one GPS point, we attribute this residual Dusky Sound deformation signal to miss alignment. The negative LOS displacement (away from the satellite) is still observed in the vicinity of the Secretary Island, however, the spatial extent is reduced.

We then assume a local source model with parameters similar to the 2003 Secretary Island earthquake and test the fault location and amount of slip, to determine the best local source model to fit Residual 2. Figure 4.17 presents results of different fault locations along strike and updip (left), and increasing amounts of slip (right), and compares them to profiles of LOS displacement from Residual 2. A maximum LOS displacement of $-25 \mathrm{~mm}$, after detrending and noise corrections, was present along profile C-C' [Figure 4.17]. The centre of the fault plane was 
first modelled at the Secretary Island epicentre, it was then shifted northeast and southwest along strike. Next, the fault centre was then shifted by trial and error to match the general pattern of negative LOS displacement in Figure 4.16 'Residual 2'. The fault centre was shifted $7 \mathrm{~km}$ west and $14 \mathrm{~km}$ north of the Secretary Island epicentre, placing the deformation further updip of the Secretary Island epicentre. The amount of slip was then varied from $1 \mathrm{~m}-3 \mathrm{~m}$ to match the shape of the residual deformation field (Figure 4.17, right). From this simplistic assessment, if the fault source is approximated well by the 2003 Secretary Island source parameters then we suggest that the centre of slip occurred northwest of the epicentre and had a maximum amount of slip in the range of $2-3 \mathrm{~m}$ (best shown in Figure 4.17, right profile $\left.\mathrm{C}-\mathrm{C}^{\prime}\right)$.

A linear-inversion was performed to independently solve for the amount of slip which best fitted the 'Residual 2' deformation field. The local source model, which fitted the pattern of negative LOS displacement (located $7 \mathrm{~km}$ west and $14 \mathrm{~km}$ north of the 2003 Secretary Island epicentre) was used (purple line Figure 4.17). The linear-inversion suggested $0.4 \mathrm{~m}$ of slip was the best fit for the data. This is significantly lower than the values modelled by visually inspecting the displacement profiles. As the linear inversion was solving for the best fit for the whole frame, and the test described above was only fitting a line, this difference is expected. From visual inspection, we can see that although the model centred northwest of the epicentre matches the region of negative LOS displacement in the selected profiles, it does not accurately represent the whole deformation field (bottom row of Figure 4.16).

To determine whether fitting a local source modelled improved the fit of the data, we remove the local deformation model (as described above with $7 \mathrm{~km}$ west and $14 \mathrm{~km}$ north epicentre and $0.4 \mathrm{~m}$ of slip) from Residual 2 to produce 'Residual 3' (bottom row of Figure 4.16). Figure 4.18 presents histograms of residual values from Figure 4.16. Observed values ('Data') are centred on $145 \mathrm{~mm}$ and have a standard deviation of $30 \mathrm{~mm}$. The values of Residual 1 have a range of c. $430 \mathrm{~mm}$ which is seen in Figure 4.18 as a low broad distribution with a median value of $22 \mathrm{~mm}$. Residual 
2 is centred around zero (median $0.5 \mathrm{~mm}$ ) and has a standard deviation of $14 \mathrm{~mm}$. Residual 3 has a median value of $-0.3 \mathrm{~mm}$ with a standard deviation of $14 \mathrm{~mm}$ (the same standard deviation as Residual 2). Figure 4.18 shows that the removal of the Dusky Sound model and Residual 1 trend removes the majority of the deformation signal. The difference between Residual 2 and Residual 3 is below the level of noise in the data. We can not determine from Figure 4.18 if the inclusion of the local source model is a better representation of the deformation field in Residual 2.

\subsection{Summary}

In this chapter, we have presented results from our preliminary investigation, a subset of interferograms, timeseries analysis, models of the Dusky Sound earthquake deformation and our attempt to model a local deformation source. Our initial investigations showed motion away from the satellite in the same epoch as the Dusky Sound earthquake, and no deformation centred on Secretary Island for the period 2015-2017, ruling out reactivated slip after the 2016 Kaikōura earthquakes [Figures 4.1 and 4.2]. To examine the local deformation signal further, we generated 96 interferograms of which we presented a subset [Figure 4.5]. These were used to produce a timeseries of cumulative displacement [Figure 4.8]. No localised deformation signal was observed following the 2007 George Sound earthquake, so reactivation following that event was ruled out [Figure 4.9]. The region near Secretary Island was relatively displaced c. $80 \mathrm{~mm}$ away from the satellite after the Dusky Sound earthquake [Figure 4.10]. To isolate this local deformation signal, we modelled the Dusky Sound earthquake [Figure 4.14], and remove this from an interferogram spanning 28 June 2009-13 August 2009 [Figure 4.16]. The residual data were detrended and topographically correlated noise was removed. A local source modelled was tested by adjusting the parameters of the 2003 Secretary Island source model [Figure 4.17], the best fitting fault location was northwest of the Secretary Island epicentre inferring an updip location. When examining histograms of the residual deformation field, we cannot determine if including a local source model improves the fit of the data 

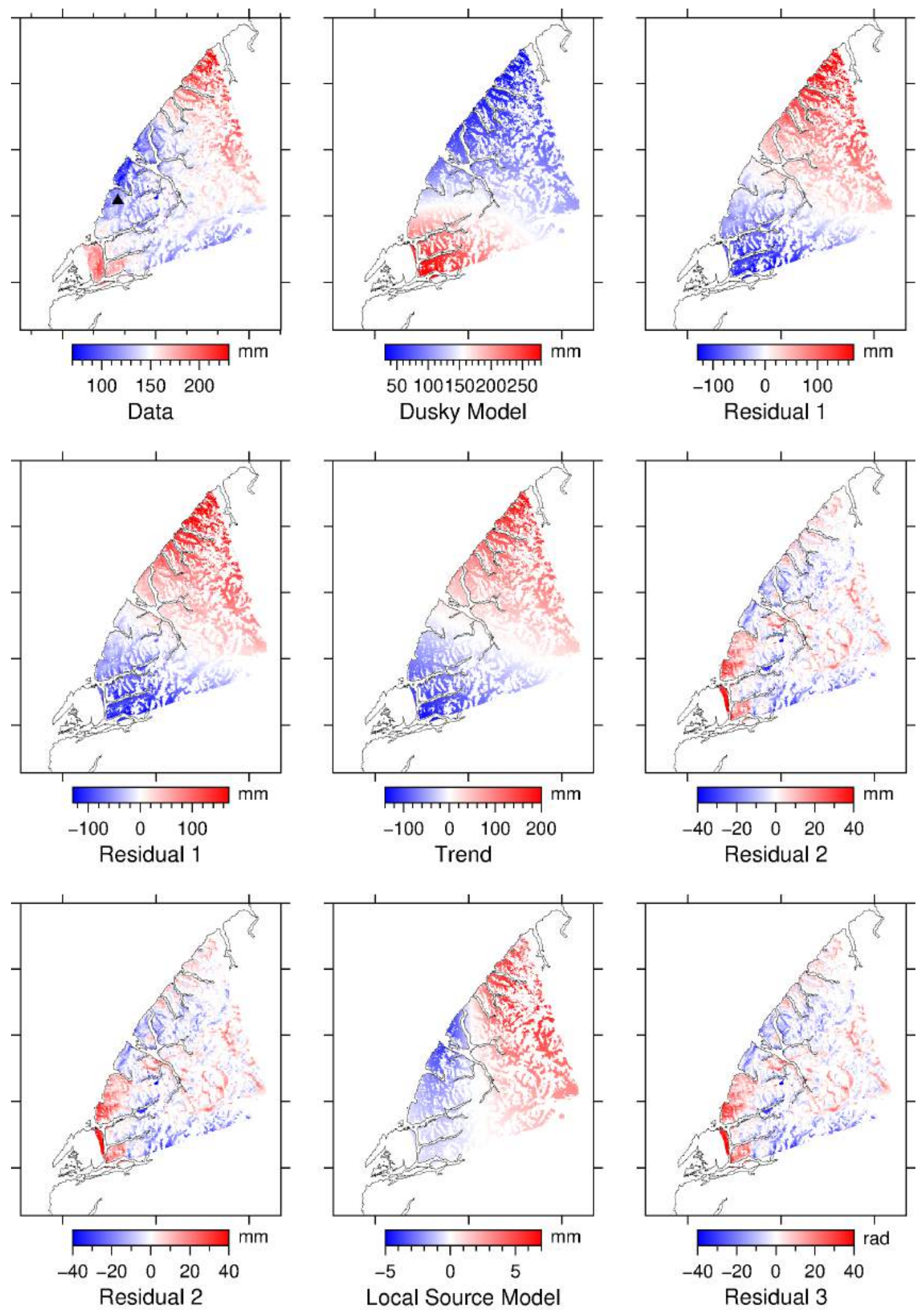

Figure 4.16: Isolating Secretary Island deformation signal. The observation (Data) had the Dusky Sound model removed producing 'Residual 1' (row one), 'Residual 1' was then detrended producing 'Residual 2' (row 2) and finally a local slip model was subtracted from 'Residual 2' producing 'Residual 3'. The local source has the slip parameters of 2003 Secretary Island earthquake however the location of maximum slip was shifted $7 \mathrm{~km}$ west and $14 \mathrm{~km}$ north, and the maximum slip was set to $1 \mathrm{~m}$. 


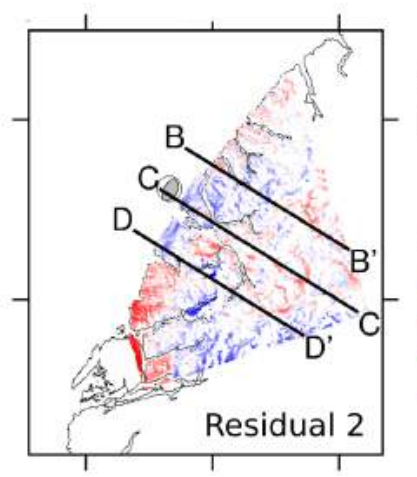

Secretary Island Slip Model:

Strike $=30^{\circ}$, Dip $=30^{\circ}$, Rake $=100^{\circ}$,

Length $=35 \mathrm{~km}$, Width $=10 \mathrm{~km}$ and

Depth $=35 \mathrm{~km}$.

Horizontal shift from epicentre Amount of Slip

$0 \mathrm{~km}$ East, $0 \mathrm{~km}$ North

$-1.0 \mathrm{~m}$

$10 \mathrm{~km}$ East, $20 \mathrm{~km}$ North

$-1.5 \mathrm{~m}$

$-5 \mathrm{~km}$ West, $10 \mathrm{~km}$ South

$2.0 \mathrm{~m}$

$7 \mathrm{~km}$ West, $14 \mathrm{~km}$ North

$-2.5 \mathrm{~m}$
$-3.0 \mathrm{~m}$
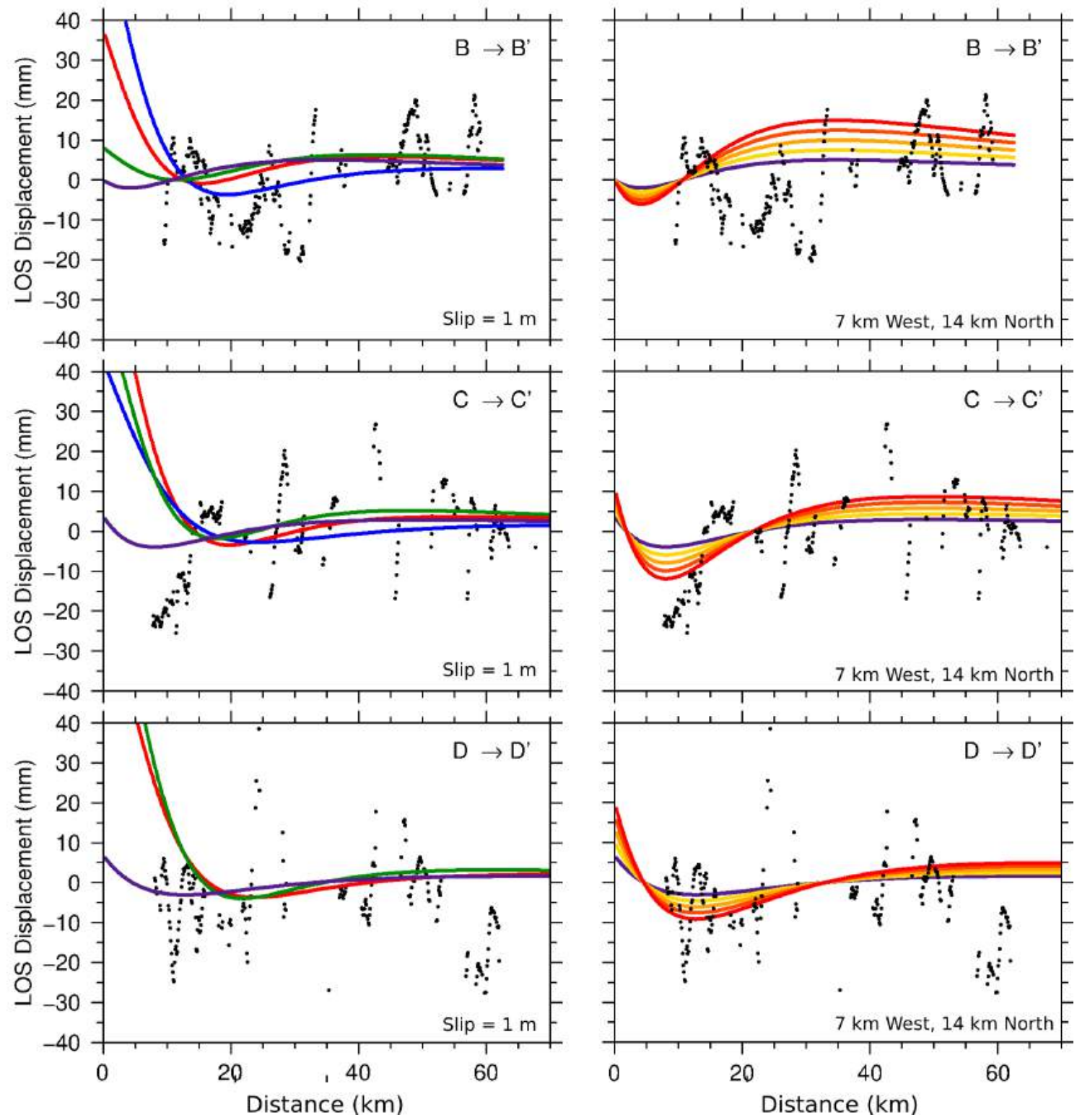

Figure 4.17: Cross-sections comparing residual displacement field and Secretary Island models. The centre of the fault plane was modelled at the 2003 Secretary Island epicentre (blue line), northeast along strike (red line) and southwest along strike (green). The pattern of motion away from the satellite was then match visually by placing the centre of the fault northwest (purple). The amount of slip was then varied to match the shape of the deformation profile (right). Slip was increased from $1.5 \mathrm{~m}$ (yellow) to $3 \mathrm{~m}$ (red). Maximum LOS displacement of $-25 \mathrm{~mm}$ was recorded along $\mathrm{C}-\mathrm{C}$ ' 


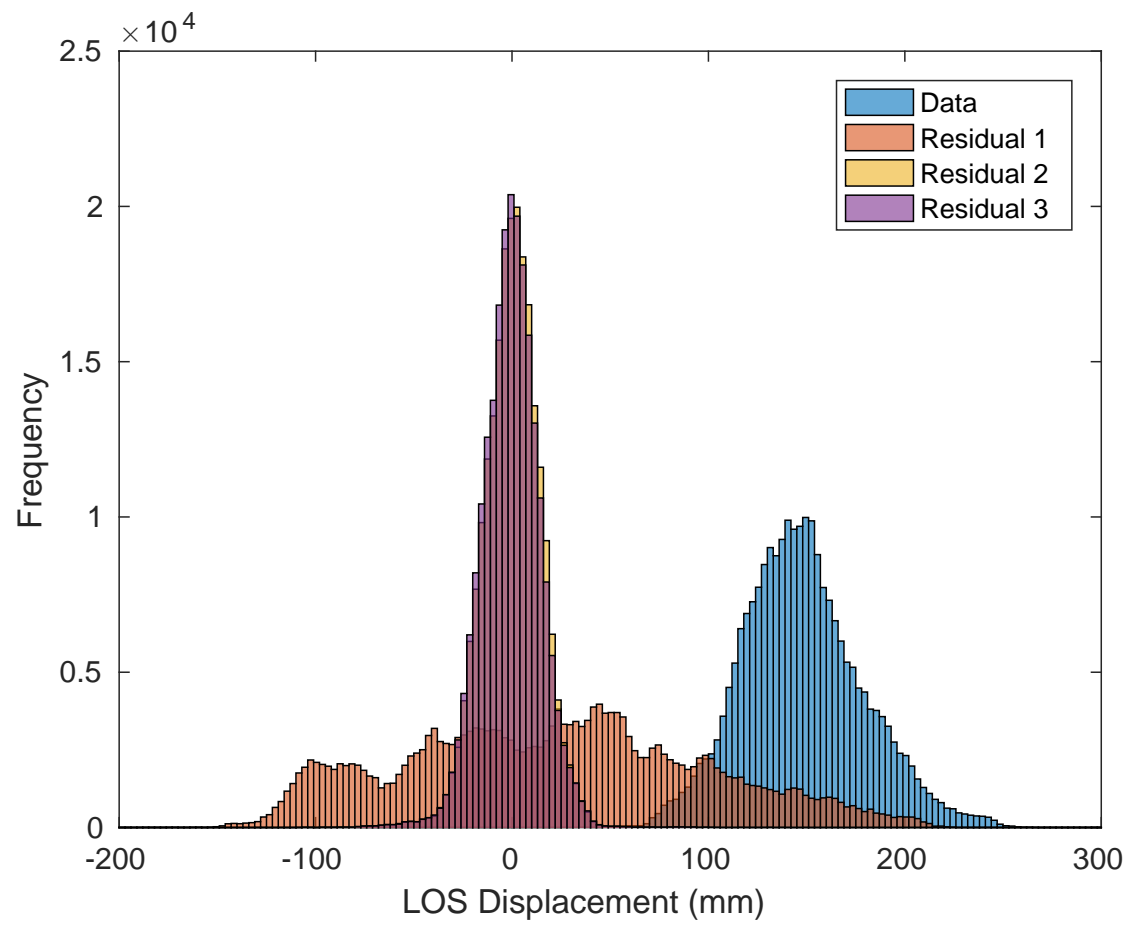

Figure 4.18: Histograms at each step of isolating the local deformation signal. Residual 1 is the observed (Data) minus the Dusky Sound model, Residual 2 is the data minus the Dusky Sound model and the trend and Residual 3 is the data minus the Dusky Sound model, the trend and a local deformation source. Refer to Figure 4.16.

[Figure 4.18]. From this analysis, we cannot rule out the presence of reactivated slip near Secretary Island following the 2009 Dusky Sound earthquake. 


\section{Discussion and Conclusions}

The previous chapter presented results from Interferometric Synthetic Aperture Radar (InSAR) processing for the purpose of identifying if there was a surface deformation signal associated with a region of reactivated slip centred on Secretary Island. In this chapter, we first discuss the limitations inherent in the dataset, how well different sources of error were mitigated during processing, and uncertainty introduced by processing methods. Second we, examine the magnitude of detectable slip given the observational geometry and the noise level of the data. The results presented in Chapter 4 are then discussed with specific reference to the constraints on timing, the magnitude of crustal movement and the spatial scale over which any deformation occurred. Our findings are compared to the post-Dusky Sound Global Positioning System (GPS) velocity field and newly identified regions of tremor in Fiordland. This chapter concludes with a comparison of these results to the triggered slip reported at George Sound and suggestions for future research.

\subsection{Limitations of the Dataset}

The inherent limits of the InSAR technique are surface preservation (i.e. changes to scattering properties of the surface causing decorrelation), phase gradient (i.e. phase changes which have a gradient steeper than one fringe change per pixel) and phase ambiguity (two-way path length $2 \pi n$, where $n$ is unknown) (Massonnet and Feigl, 1998). As addressed in Sections 3.1 and 3.3, temporal decorrelation due to changes in the scattering properties of the ground surface are expected when such coherence was observed in this study [Figure A7]. The lack of coherence in regions with variable snowfall provided challenges in phase extraction. This was overcome to a 
certain extent through the application of a uniform coherence mask and the forced unwrapping of remaining pixels (as described in Section 3.3). Extracting phase from regions with low coherence produced an overall increase in short-wavelength noise. Steep phase gradients from tectonic sources did not affect the result as no surface ruptures, or other feature causing steep phase changes over distances smaller than one pixel were expected in the dataset. The application of pre-unwrapping interpolation mitigated the effects of unwrapping errors due to phase ambiguity (Section 3.3).

Synthetic Aperture Radar (SAR) acquisition parameters add further limitations including temporal (frequency of acquisitions), spatial (ground surface extent and pixel resolution) and orbital (platform drift). In this study, the frequency of SAR acquisitions determined whether a deformation signal associated with triggered slip could be temporally constrained to immediately before, or after, an earthquake. For track 348, acquisitions bracketing the George Sound earthquake were made 23 days before and 26 days after the earthquake, and for the Dusky Sound earthquake, bracketing acquisitions were made 17 days before and 29 days after. This meant that any detected slip could only be temporally constrained to within a c.50 day window in each case. By contrast, for the purpose of modelling the Dusky Sound earthquake co- and post-seismic deformation the timing of acquisitions provided adequate coverage.

Our ability to quantify noise levels and detrend the data were limited by the spatial extent of the available SAR frames. The detrended data were biased by the surface deformation within the frames and introduced errors at the edges of the frames [Figure 4.6]. When calculating the linear-ramp for track 348, frame 6260, approximately one-third of the fame was dominated by the local deformation field surrounding Secretary Island. These errors associated with detrending were propagated into time series analysis and could be seen along the southwest edge of the frame [Figure 4.8]. The spatial resolution of the data were reduced to one-quarter of the Gaussian filter wavelength $(400 \mathrm{~m})$, producing a $100 \mathrm{~m}$ horizontal ground resolution (as in Section 3.3). For the purpose of investigating a deformation signal 
with a surface expression on the order of tens of kilometres, this pixel resolution was adequate.

One of the largest limitations for producing high coherence interferograms was the accuracy of the ALOS1 repeat orbit trajectories. As shown in Figures 2.3 and 4.7, many pairs with long temporal baselines were formed as the orbital drift of ALOS1 prohibited short temporal pairs. The low coherence in many images arose from the long temporal separations, which averaged 539 days (1.47 years). The lack of pairs with short-temporal baselines also limited our ability to discriminate when reactivated slip could have occurred. Of the 96 interferograms, 52 spanned intervals longer than 1 year, 50 spanned the Dusky Sound earthquake and 42 spanned both the George Sound and Dusky Sound earthquakes. As the majority of interferograms included deformation from the George Sound and Dusky Sound earthquakes, smallscale displacements were overwhelmed by relatively large displacements occurring within the duration of the interferogram.

Location-specific limitations restricted continuous sample-spacing, forming data gaps and under-sampling of phase. The steep topography in Fiordland resulted in shadow regions that the radar signal could not reach [Figure 3.16]. Water bodies and seasonal snow restricted regular sample-spacing. These features caused phase discontinuities, which when unwrapped were expressed at steps in line-of-sight (LOS) displacement. With the implementation of the pre-unwrapping interpolation, could be addressed, improving the final results (see Figures A13, A14, A15, A16 and A17). For future InSAR processing in New Zealand, we recommend the use of pre-unwrapping interpolation to improve computation time and reduce unwrapping errors in adverse conditions.

\subsection{Uncertainty Introduced from Methodology}

The method used to isolate the local deformation centred on Secretary Island following the Dusky Sound earthquake has a number of sources of uncertainty which are outlined below. 
1. A static shift was performed to tie the InSAR- and GPS-derived co-seismic deformation fields (Section 3.5). As track 348, frame 6260 contains no continuous GPS stations and the campaign GPS sites coincided with regions of low coherence, only measurements from one site (DF4L) were applicable. The uncertainty of the co-seismic displacement measurement at DF4L was $4 \mathrm{~mm}$ east, $5 \mathrm{~mm}$ north and $12 \mathrm{~mm}$ vertically (Beavan et al., 2010). When tying the InSAR-derived deformation field to the GPS site, these uncertainties are inherited.

2. The Dusky Sound model was derived using a single uniform fault plane which does not totally account for the near-field deformation [Figure 4.14]. In particular, this simplified the fault geometry may not accurately represent the deformation field near to Secretary Island (discussed further in the next section).

3. When isolating the Secretary Island signal in 2009 [Figure 4.16], the trend was only calculated using one frame (track 348, frame 6260), which, as described above, was dominated by deformation from the Dusky Sound earthquake. The calculated trend is sensitive to details of the Dusky Sound co-seismic deformation model subtracted from the data. Any error in the Dusky Sound co-seismic deformation may add or strip out localised deformation signal if the modelled doesn't accurately represent the deformation field [Figure 4.16].

4. We made the assumption that the residual local deformation signal centred on Secretary Island could be modelled with a source similar to that of the 2003 Secretary Island earthquake, determined with geodetic modelling by Reyners et al. (2003). Our results are therefore contingent on assumption.

When searching for a deformation signal on the order of tens of millimetres, error introduced at any one of these four steps will propagate through and may remove the deformation signal out of the final result. 


\subsection{Comparison of Dusky Sound Deformation Model}

As described above, our ability to determine whether reactivated slip occurred relies on the quality of the deformation model removed from the data. We compare our Dusky Sound co-seismic deformation model results to those of Beavan et al. (2010) to differences arising from contrasting modelling approaches.

The primary purpose of re-analysing the Dusky Sound deformation model was to produce a model which accurately removed the deformation associated with the 2009 earthquake so that the deformation signal associated with a triggered slip source could be effectively isolated. For this study, accurately representing the farfield deformation $(>40 \mathrm{~km})$ was of greater importance than capturing the near-field deformation. In contrast, the study by Beavan et al. (2010) addressed in finer detail the spatial and temporal evolution of the fault geometry and subsequent after-slip distribution. Using a Bayesian modelling approach, we have completed an extensive analysis of a simplistic fault model, whereas Beavan et al. (2010) used a kinematically more complex model but undertook less statistical analysis. In this study, we solved for 12 parameters, with bounds which did not limit the solution, and iterated the solution 1,000,000 times. Beavan et al. (2010) fixed the fault location, depth, and size and then solved for slip on each $5 \mathrm{~km}$ grid cell on a $120 \times 50 \mathrm{~km}$ fault patch. In both studies, InSAR data were inverted simultaneously with 27 campaign GPS (sGPS) and 14 continuous GPS (cGPS) data points. Similar to this study, Beavan et al. (2010) found that the inclusion of sGPS was required to stabilise the inversion. Both studies down-sampled interferograms to $100 \mathrm{~m}$ pixel size and performed quad-tree sub-sampling; Beavan et al. (2010) reduced the number of data points to 50-450 for each InSAR image, while this study used c. 1000 for each InSAR frame. Each time step in this study was solved for using the InSAR data acquired on that day and the GPS data. Beavan et al. (2010) included InSAR data from all three acquisition dates in the inversion, but assigned different weights according to the epoch; this meant that those results included contributions from different observation geometries at each time step. 
As presented in Table 4.1, the moment magnitudes for the co-seismic and the combined co-seismic +8 days of post-seismic were similar $( \pm 0.05)$. Beavan et al. (2010) concluded that 5-6 m of slip occurred on an $80 \times 50 \mathrm{~km}$ fault patch, with a maximum slip at a depth of $30-40 \mathrm{~km}$ in the epicentral region, decreasing to $17 \mathrm{~km}$ southwest along strike. Our results indicate $4.4 \mathrm{~m}$ of slip on a $60 \times 55 \mathrm{~km}$ patch with the depth to the bottom edge of the fault plane $30 \mathrm{~km}$. Both studies placed the location of maximum slip c. $30 \mathrm{~km}$ southwest of the epicentre. We compare the residuals of the two model in Figure 5.1. Overall, we find that the two models produce similar results with the model of Beavan et al. (2010) illuminating finer details of the evolution of after-slip and this study characterising the fault parameters based on posterior probability density functions (PDFs).

\subsection{Magnitude of Detectable Slip}

To determine what magnitude of slip is expected to be detectable given the observation geometry and the noise in the data, we have modelled a hypothetical slip source and compared the expected east, north, up and LOS deformation fields. Figure 5.2 presents a model of $1 \mathrm{~m}$ of slip on a pure thrust fault plane in the location of the 2003 Secretary Island earthquake. As the rake of the 2003 Secretary Island earthquake was $100^{\circ}(($ Reyners et al., 2003)), the sense of movement on a reactivated patch is assumed here also to be predominantly reverse and can be approximated for the purpose of this comparison as being pure reverse-slip. Figure 5.2 shows horizontal displacement towards the fault (i.e. westward), with uplift along the upper edge of the slipping patch and subsidence along the lower edge. Given the ascending and right-looking observation geometry along track 348, ground motion west, south and up, moves the surface of the earth closer to the satellite. As Figure 5.2 illustrates, the modelled westward and downwards motion largely cancel each other out when observed in the LOS direction. This model suggests that a large amount of uplift (of the order of c. $120 \mathrm{~mm}$ for $1 \mathrm{~m}$ of slip) is generated offshore along the tip of the blind thrust, which is not able to be measured by InSAR. The onshore portion of the 

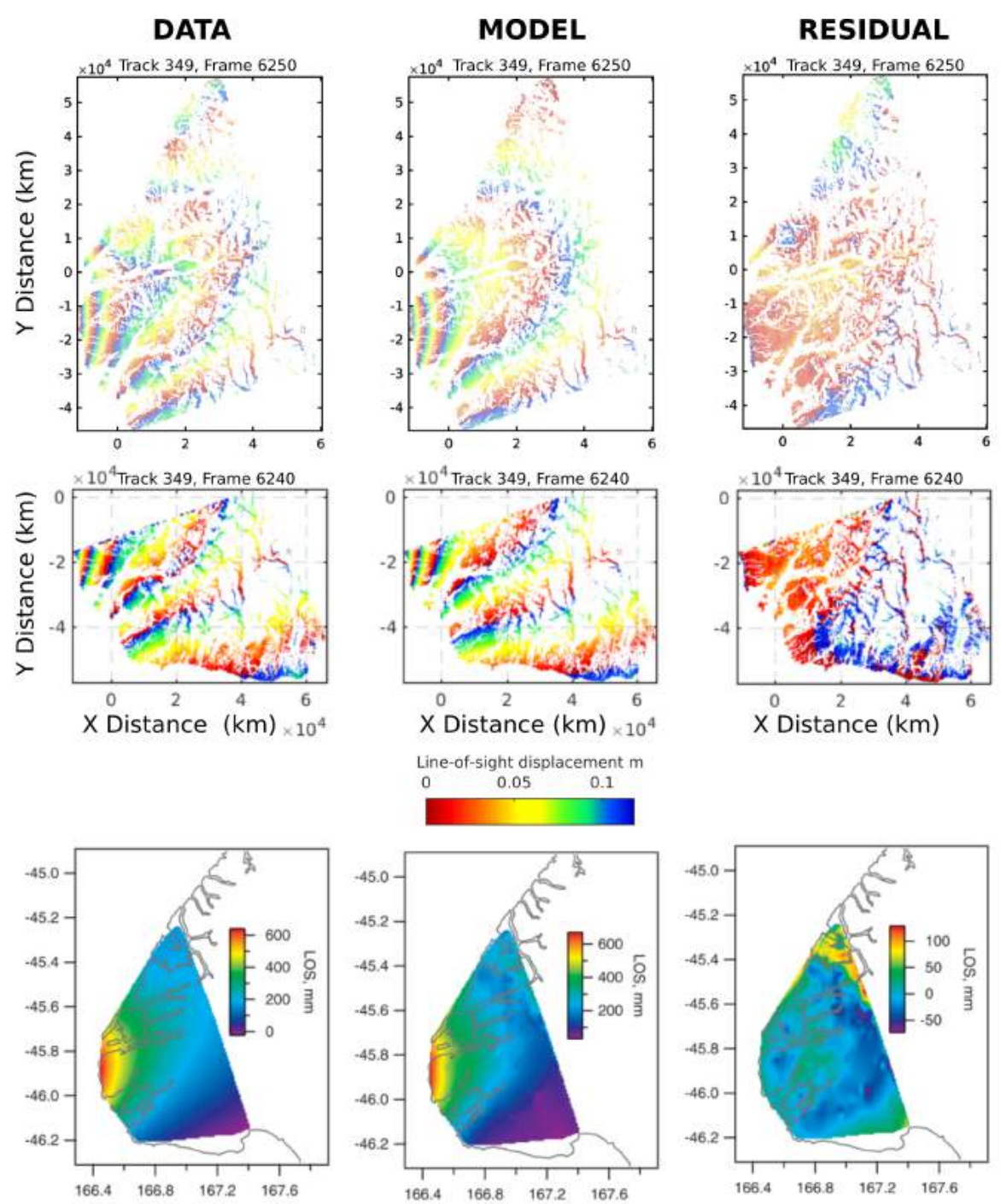

Figure 5.1: Comparison of modelling from this study (top) and that of Beavan et al. (2010) (bottom). The data in Beavan et al. (2010) are a smoothed version of quad-tree sampling of the original data. We can see that the general pattern of deformation is similar, however, greater smoothing has been applied to the models of Beavan et al. (2010). The residual plots indicate that the model from this study has similar misfit in the near-field, with Beavan et al. (2010) producing misfit on the order of $50 \mathrm{~mm}$ in the epicentral region. Our model produces nearly to $100 \mathrm{~mm}$ of misfit in the south frame (6240) which is not observed in Beavan et al. (2010). Other regions of significant misfit in the model of Beavan et al. (2010) correlate to the regions of low coherence which were excluded in this study. 
Slip on a pure thrust fault

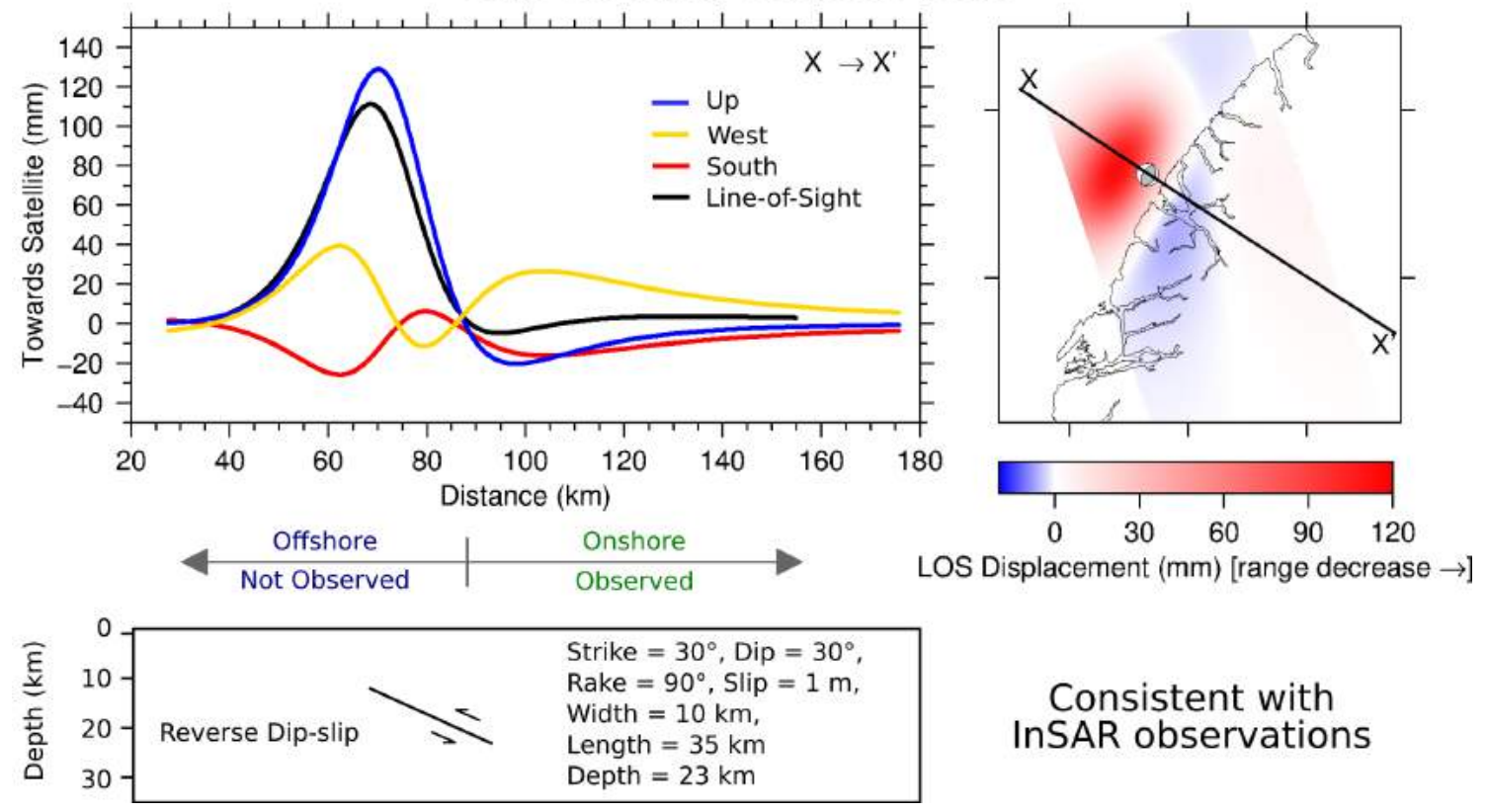

Figure 5.2: Tectonic interpretation of a blind thrust source causing surface deformation near Secretary Island. The portion of the deformation signal observed InSAR, given the ALOS1, track 348 geometry is limited to the region onshore of westward motion and subsidence. When viewed in LOS geometry, $1 \mathrm{~m}$ of slip on the fault results in motion away from the satellite of c. $5 \mathrm{~mm}$.

deformation field, able to be measured using InSAR, only undergoes a small amount of motion away from the satellite where subsidence at the bottom edge of the fault plane occurs. For $1 \mathrm{~m}$ of slip on the fault, the onshore portion of the deformation field moves down and west, producing a small LOS signal of c. $5 \mathrm{~mm}$ away from the satellite. Given the residual scatter in the observations $( \pm 15 \mathrm{~mm})$, we determine that reactivated slip below of $1 \mathrm{~m}$ cannot be detected with the available dataset [Figure 3.16 and 4.17].

\subsection{Triggered Slip near Secretary Island}

The data used in this study do not indicate reactivation of slip on the 2003 Secretary Island rupture patch following either the 2007 George Sound or 2016 Kaikōura earthquakes [Figures 4.2 and 4.17]. The local deformation signal expected for such slip, was not detected in ALOS1 data between 21 December 2006 and 26 December 2008 [Figures 4.7, 4.8, and 4.9]. Preliminary analysis of ALOS2 for the period 26 
November 2014 to 26 November 2017 also did not present any evidence for localised deformation signals near Secretary Island [Figure 4.2].

We did, however, find evidence of a temporal window in which reactivated slip following the 2009 Dusky Sound earthquake could have occurred [Figure 4.7]. By manually eliminating interferograms with and without the local deformation signal near Secretary Island, we found that slip may have occurred during the period of 28 June 2009 and 13 August 2009 [Figure 4.7]. There is additional evidence for a local deformation signal in the following periods: 26 December 2008-10 February 2009; 29 December 2009-13 February 2010; and 16 August 2010-1 October 2010 [Figures 4.8 and 4.10]. In each case, there was one interferogram which included a deformation signal consistent with the Secretary Island area undergoing movement away from the satellite [Figures 4.7].

The spatial extent of the Secretary Island deformation signal, c. $50 \mathrm{~km}$ parallel and c. $25 \mathrm{~km}$ perpendicular to the coastline, was measured from the cumulative time series [Figure 4.9]. After removing the Dusky Sound deformation model and detrending the modelling, the surface extent of deformation was smaller but still centred near Secretary Island [Figure 4.16]. The maximum recorded subsidence was c. $25 \mathrm{~mm}$, but the residual deformation included topographically-correlated noise [Figure 3.16].

When fitting a forward model of deformation produced by a source similar to the 2003 Secretary Island earthquake to the observed deformation field, we found that slip was required to be centred offshore, northwest of the 2003 Secretary Island epicentre [Figures 4.17]. The preferred location of triggered slip northwest of the Secretary Island epicentre may indicate that slip on a shallower portion of the subduction interface than the 2003 earthquake. Simple forward modelling suggests that the amount of slip required to produced the observed subsidence is in the range of 1-3 m, corresponding to a moment magnitude in the range of $M_{w} 6.6-6.9$. This amount of slip is of the same order of magnitude as occurred in the 2003 Secretary Island island earthquake which produced $2.4 \pm 0.5 \mathrm{~m}$ of slip in a $M_{w} 7.2$ event (Reyners et al., 2003). 


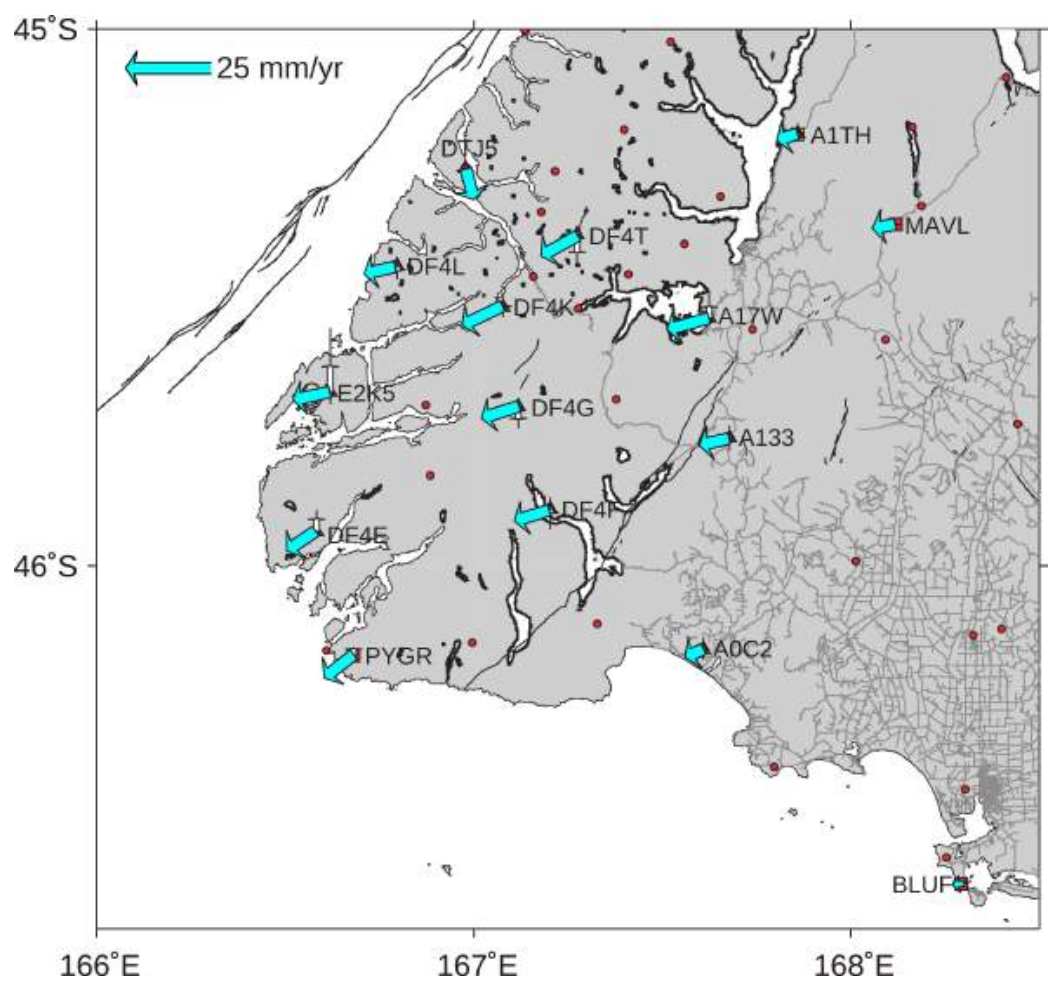

Figure 5.3: Fiordland horizontal velocity field following the Dusky Sound earthquake, fixed to the Australian Plate reference frame. Data from sGPS and cGPS for the period 2010 to 2014 processed by Sigrun Hreinsdóttir (pers. comm 2018). Site DTJ5 located on Secretary Island has a southeast velocity vector that differs from those of neighbouring sites. Figure adapted from Sigrun Hreinsdóttir (pers. comm 2018)

We can also consider whether triggering of slip could have occurred by transient stresses from the passage of seismic waves from the Dusky Sound earthquake, or by static stress changes related to the long term stress field (Freed, 2005). Fry et al. (2010) noted that the seismic waves from the Dusky Sound earthquake had southward directivity, meaning that radiated seismic energy was preferentially directed away from Secretary Island. As the slip patch we inferred following the Dusky Sound earthquake is located northeast of the Dusky Sound epicentre, we suggest slip is then unlikely to have been triggered by passing seismic energy, similar to the George Sound case (Hamling and Hreinsdóttir, 2016). However, further analysis is required to substantiate or disprove this conclusion. 


\subsection{Comparison with Independent Observations}

The unpublished post-Dusky Sound velocity field for 2010-2014 obtained by Hreinsdóttir (pers. comm., 2018), indicated that the sGPS site on Secretary Island moved in a southeast direction, while the remainder of sGPS sites moved southwest [Figure 5.3]. The modelled deformation, assuming a reverse-slip source similar to that of the 2003 Secretary Island earthquake, suggests northwest motion at DTJ5 that is not observed in the GPS velocity field [Figures 4.3 and 5.2]. Figure 5.4 presents an alternative model which agrees with the GPS-derived velocity field, but not with the InSAR observations. Slip on a dextral strike-slip fault, such as the Alpine Fault, northeast of the 2003 Secretary Island epicentre produces southeast motion at DTJ5 on Secretary Island. In both reverse-dipslip and dextral-strikeslip models, the magnitude of LOS displacement produced by $1 \mathrm{~m}$ of slip is on the order of $5-20 \mathrm{~mm}$, which is below the scatter in the observations [Figures 5.2 and 5.4]. To properly quantify the three-dimensional displacement and timing of slip GPS data must be used in conjunction with InSAR.

Romanet and Ide (2019) recently reported regions of seismic tremor in Fiordland during the period 2005-2016. This new catalogue is not likely to be capturing all tremor in the region, as the seismic station density is sparse (Romanet and Ide, 2019). Nevertheless, the clusters of tremor were located southeast of the Secretary Island epicentre toward the head of Doubtful Sound, near the 2007 George Sound rupture patch and between the heads of George and Caswell Sounds [Figure 5.5]. The occurrence of tremor is an independent line of evidence in support of aseismic slip in the vicinity of Secretary Island (Rogers and Dragert, 2003). The tremor cluster near Secretary Island delineates the sharp change in strike of the plate boundary and steepening of the subducting plate (Eberhart-Phillips and Reyners, 2001, Reyners et al., 2002). The location of tremor to the southeast of the 1989, 1993 and 2003 earthquake ruptures, may indicate the location of tremor and episodic slip on a deeper portion of the plate interface (Romanet and Ide, 2019).

Three episodes of tremors were detected on 21 April 2009 near Secretary 


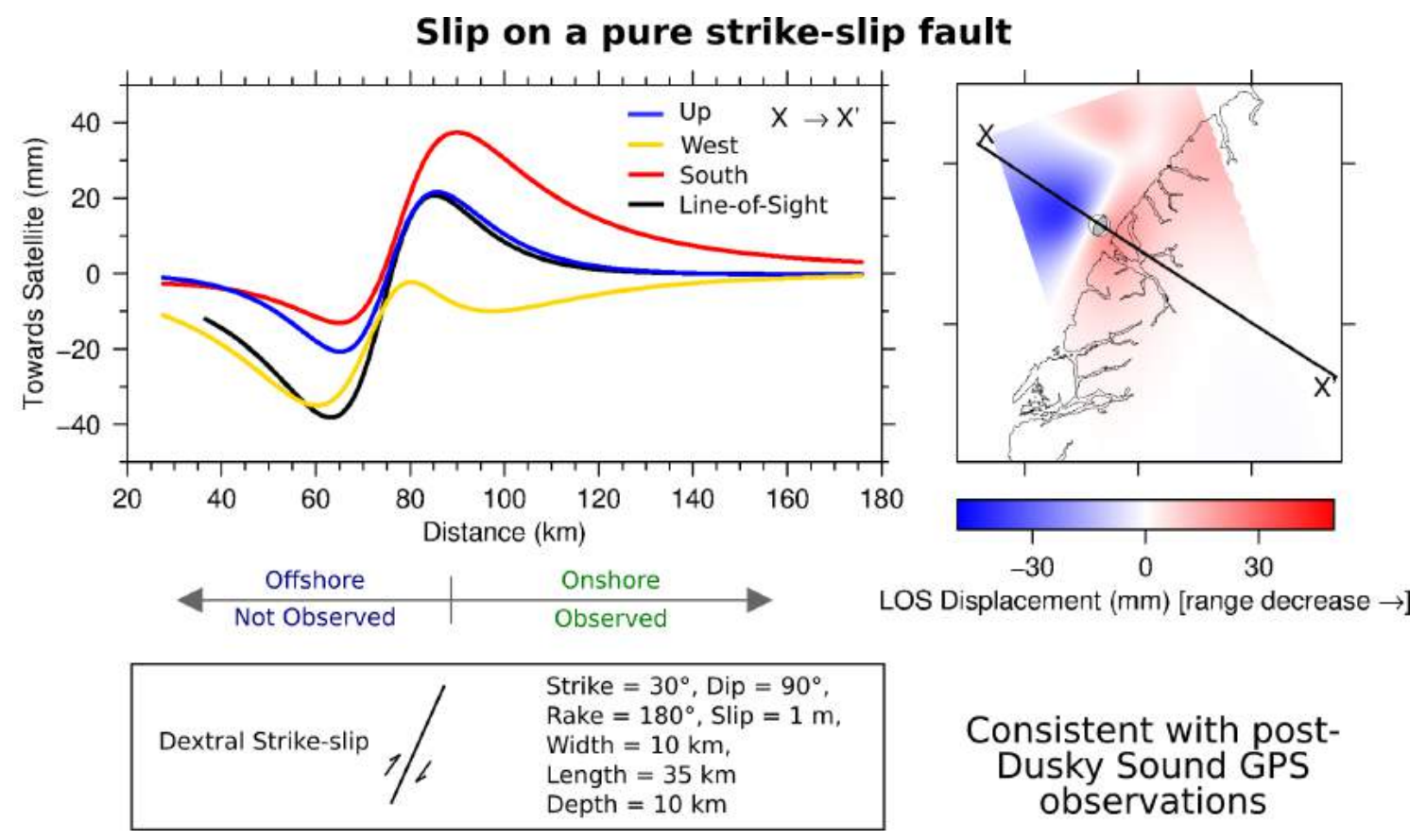

Figure 5.4: Alternative tectonic deformation model which agrees with post-Dusky Sound GPS observations from Sigrun Hreinsdóttir (pers. comm 2018). With this source model site DTJ5 located on Secretary Island has a southeast. This produces LOS motion towards the satellite which is not observed in the InSAR measurements.

Island prior to the 2009 Dusky Sound earthquake (Romanet and Ide, 2019). Following the Dusky Sound earthquake, tremor near Secretary Island was identified on 19 August 2009, 8 February 2010, and 8 November 2010 (Romanet and Ide, 2019). The tremor on 8 February 2010 corresponds to the potential slip window of 9 December 2009-13 February 2010 inferred from the InSAR analysis with this study, while the tremor on 8 November 2010 occurs within the 16 August 2010-1 October 2010 slip window (Figure 4.7). The tremor located near George Sound occurred after the Dusky Sound earthquake, but not within the period of triggered afterslip identified by Hamling and Hreinsdóttir (2016).

\subsection{Comparison with Triggered Slip at George Sound}

The method we have used to isolate the surface deformation signal from triggered slip differs from the method of Hamling and Hreinsdóttir (2016) in three key ways: 

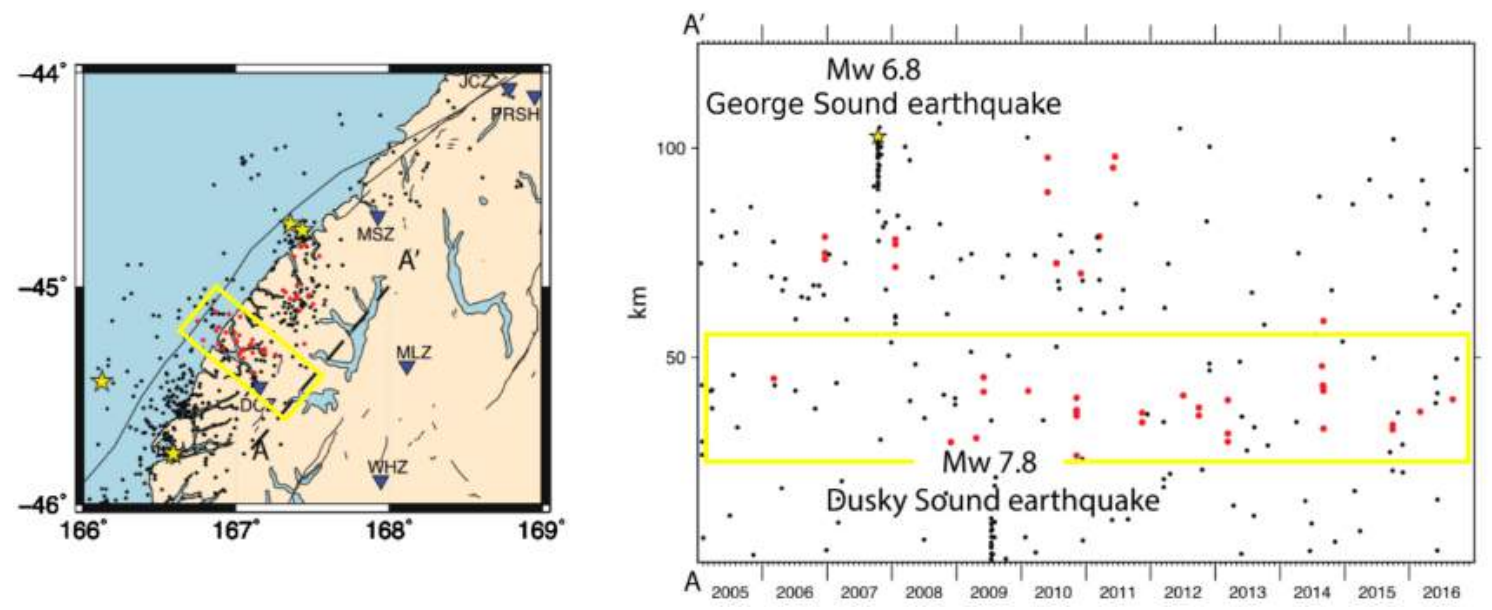

Figure 5.5: Recently reported tremor in Fiordland (red dots) (Romanet and Ide, 2019). Yellow box indicates the tremor located near the 1989, 1993 and 2003 earthquakes. Black dots are earthquakes $M_{w}>4$ and yellow stars $M_{w}>6$ between 20052016.

InSAR processing and time series analysis were completed with the Stanford Method for Persistent Scatterers (StaMPS); the Dusky Sound model of Beavan et al. (2010) was subtracted from the cumulative displacement for nine months following the Dusky Sound earthquake; and a distributed slip model was used to estimate the magnitude of slip. Prior to modelling, noisy pixels were smoothed by down-sampling pixels within $20 \mathrm{~km}$ of the George Sound earthquake to $2 \mathrm{~km}$ spacing, and pixels at greater distances to $5 \mathrm{~km}$ spacing. The same corrections, for removal of linear trends and topographically correlated noise, were applied in this study, but, the corrections of Hamling and Hreinsdóttir (2016) were made to each image in the time series, rather than to individual interferograms. Hamling and Hreinsdóttir (2016) also used sGPS and cGPS data, beyond those published by Beavan et al. (2010).

The reactivated slip results of Hamling and Hreinsdóttir (2016) showed surface deformation with a maximum amplitude of c. $100 \mathrm{~mm}$ towards the satellite, with a lateral extent of $20-25 \mathrm{~km}$ along strike. The maximum magnitude of the detected slip in this study, after detrending and noise corrections, was c. $25 \mathrm{~mm}$, and the lateral extent was c. $50 \mathrm{~km}$ along strike, and c. $25 \mathrm{~km}$ perpendicular to strike. As shown in Figure 5.6 of Fry et al. (2010), the rupture location estimated from the aftershock distribution of the George Sound earthquake was smaller than that of the Secretary Island earthquake. Therefore it follows, that the patch reactivated 
near George Sound may also be smaller and generate a deformation pattern with a smaller spatial extent. As the assumed rakes only differ by $10^{\circ}$, we expect that the surface deformation from reactivated slip on the George Sound and Secretary Island rupture planes would have similar spatial distribution. Figure 5.2 indicates that the equivalent motion towards the satellite observed at George Sound would have occurred offshore in the theoretical reverse-dipslip model of slip near Secretary Island.

\subsection{Future Research}

This study reinforces the need for increased density of ground-based measurements in the southwest of the South Island. Both the GPS dataset and the tremor catalogues were compromised by the constraints of a sparse network and the infrequent measurements of sGPS sites. To estimate long-term and incremental deformation with InSAR it would be benefial to integrate sGPS data (which is yet to be published) into a three-dimensional deformation field. In New Zealand, the national sGPS network is occupied approximately once every eight years (Beavan et al., 2016), which Beavan et al. (2010) noted is not often enough to accurately measure co-seismic displacements. As in the case of the Dusky Sound earthquake displacement, multiple earthquakes had occurred since the previous sGPS occupation, and the accumulated co-seismic displacements from previous earthquakes, at some sites, had to be estimated before the Dusky Sound offset could be determined (Beavan et al., 2010). These ground-based measurements are vital for the full utilisation of satellite-based observations of deformation, and more frequent occupation of sGPS sites, and seamless data availability would provide the opportunity to better constrain surface deformation in New Zealand.

As we cannot rule out the presence of triggered slip, future analysis is required to determine why reactivation George Sound occurred following the Dusky Sound earthquake, and why it was not clearly observed in this study in the vicinity of Secretary Island. As the George Sound earthquake happened more recently one 


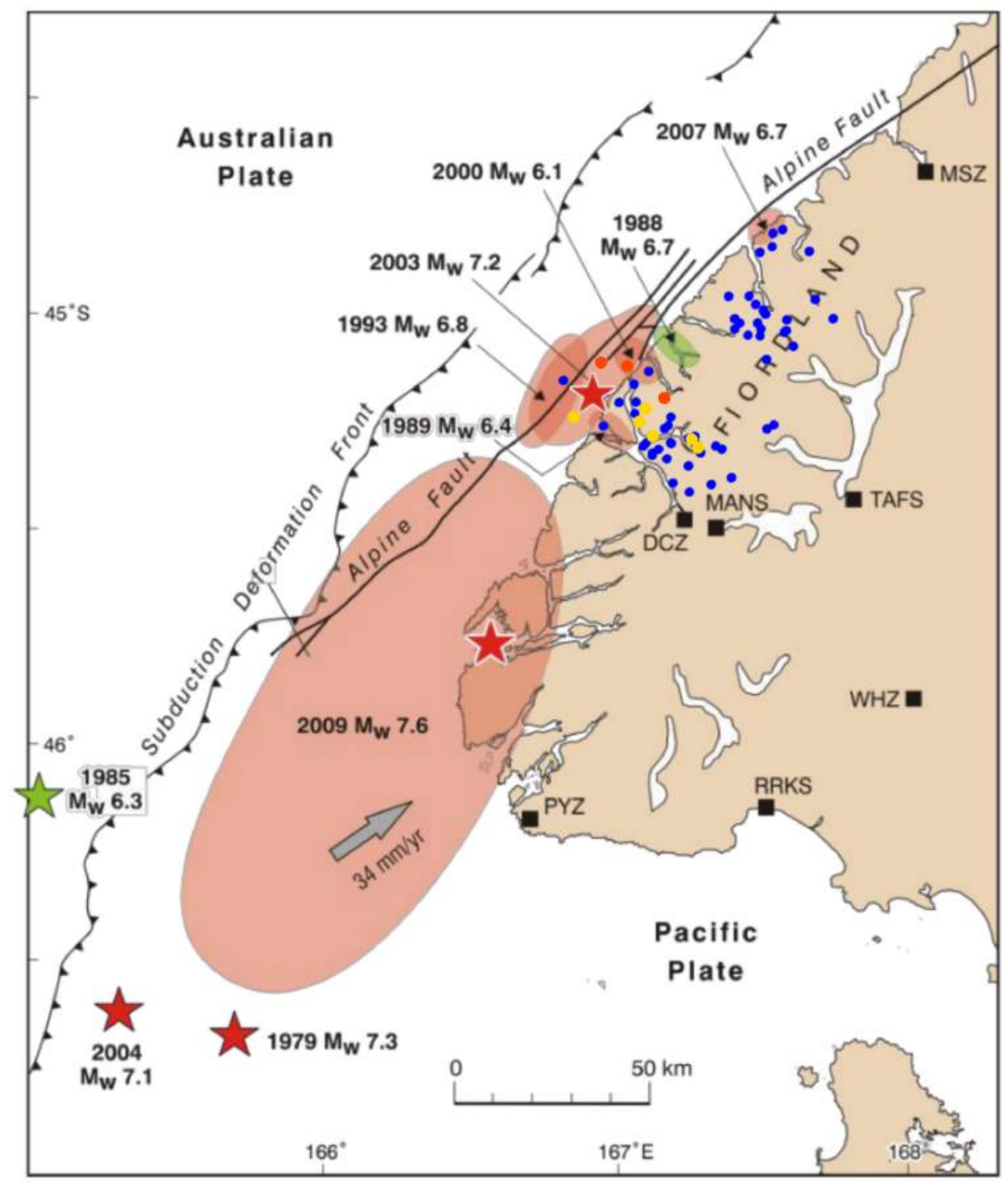

Figure 5.6: Figure from Fry et al. (2010) overlaid with the tremor catalogue of Romanet and Ide (2019). Tremor on 21 April 2009 (prior to the 2009 Dusky Sound earthquake) is plotted as orange circles, between the Dusky Sound earthquake and 2011 is plotted in yellow, and all other tremor events plotted in blue. Generally, tremor is located in three clusters 1) inland from 2003 Secretary Island rupture 2) inland from 2007 George Sound rupture and 3) between the two previously listed clusters. The predicted rupture patches based on 3 months of aftershocks for each earthquake are shaded in pink. 
possibility is that the rupture surface of the 2003 Secretary Island earthquake had healed before the Dusky Sound earthquake, but not that of the 2007 George Sound rupture. An alternative interpretation is that past events reduced the stress on the Secretary Island section of the plate interface so that it was not optimally loaded to slip again. We suggest a further detailed analysis of the three-dimensional deformation field is required to constrain surface deformation so that future research can address the interactions between different sections of the plate boundary in Fiordland.

\subsection{Summary and Conclusions}

We used L-band SAR data from ALOS1 and ALOS2 to investigate the presence of localised surface deformation centred on Secretary Island following the 2007 George Sound, 2009 Dusky Sound and 2016 Kaikōura earthquakes. We conclude that reactivation of after-slip in the vicinity of the 2003 Secretary Island earthquake did not occur following the 2007 George Sound and 2016 Kaikōura earthquakes. However, we cannot rule out aseismic slip from a source similar to that of the 2003 Secretary Island earthquake following the 2009 Dusky Sound earthquake.

Our ability to resolve the source of local deformation near Secretary Island is hindered by imprecise knowledge of the location of potential slip sources offshore and by the observation geometry of ALOS1. With the two proposed deformation models of pure dipslip, approximating slip on a source similar to the 2003 Secretary Island earthquake, and pure strikeslip, approximating slip along the Alpine Fault, the angle of incidence of the radar means that the equivalent LOS displacement is on the order of $5-20 \mathrm{~mm}$ for $1 \mathrm{~m}$ of slip. As the scatter in the observations, after the removal of long-wavelength ionosphere and short-wavelength atmosphere noise, is on the order of $\pm 15 \mathrm{~mm}$, the amount of slip from an offshore source would need to be greater than $1 \mathrm{~m}$ to be detected within this dataset. The large baseline drift of ALOS1 (c. $6 \mathrm{~km}$ in 2.5 years), prohibited the generation of interferograms with short temporal separations, leading to low coherence images and poor temporal 
constraints on the timing of deformation.

InSAR observations indicated surface motion (c. $-80 \mathrm{~mm}$ ) away from the ALOS1 satellite near Secretary Island following the 2009 Dusky Sound earthquake. We identified a time window between 28 June 2009 and 13 August 2009 where local deformation in the vicinity of Secretary Island was detected, with potential for additional deformation between December 2009 and October 2010. Assuming a model with a similar source as the 2003 Secretary Island earthquake, we place the deformation offshore, northwest of the 2003 Secretary Island epicentre. This location indicates slip on an updip portion of the subduction interface, as observed for the reactivated afterslip at George Sound following the Dusky Sound earthquake (Hamling and Hreinsdóttir, 2016). The modelled fault plane with an area of c. 350 $\mathrm{km}^{2}$, and an average slip of 1-3 m, can account for the observed motion away from the satellite of c. $25 \mathrm{~mm}$ at Secretary Island (after corrections applied). Recently reported tremors in Fiordland inferred to be located downdip of the 2003 Secretary Island earthquake provide support for the presence of episodic slip along this section of the Puysegur Subduction Zone (Romanet and Ide, 2019). 


\section{Bibliography}

Anderson, H. and Webb, T. (1994). New Zealand seismicity: Patterns revealed by the upgraded National Seismograph Network. New Zealand Journal of Geology and Geophysics, 37(4):477-493.

Bagnardi, M. and Hooper, A. (2018). Inversion of surface deformation data for rapid estimates of source parameters and uncertainties: A Bayesian approach. Geochemistry, Geophysics, Geosystems, 19(7):2194-2211.

Barnes, P. M. (2009). Postglacial (after $20 \mathrm{ka}$ ) dextral slip rate of the offshore Alpine Fault, New Zealand. Geology, 37(1):3-6.

Beavan, J., Samsonov, S., Denys, P., Sutherland, R., Palmer, N., and Denham, M. (2010). Oblique slip on the Puysegur subduction interface in the 2009 July MW 7.8 Dusky Sound earthquake from GPS and InSAR observations: Implications for the tectonics of southwestern New Zealand. Geophysical Journal International, 183(3):1265-1286.

Beavan, J., Wallace, L. M., Palmer, N., Denys, P., Ellis, S., Fournier, N., Hreinsdottir, S., Pearson, C., and Denham, M. (2016). New Zealand GPS velocity field: 1995-2013. New Zealand Journal of Geology and Geophysics, 59(1):5-14.

Berardino, P., Fornaro, G., Lanari, R., and Sansosti, E. (2002). A new algorithm for surface deformation monitoring based on small baseline differential SAR interferograms. IEEE Transactions on Geoscience and Remote Sensing, 40(11):23752383.

Berryman, K. R., Cochran, U. A., Clark, K. J., Biasi, G. P., Langridge, R. M., and Villamor, P. (2012). Major earthquakes occur regularly on an isolated plate boundary fault. Science, 336(6089):1690-1693.

Bevis, M., Businger, S., Herring, T. A., Rocken, C., Anthes, R. A., and Ware, R. H. (1992). GPS meteorology: Remote sensing of atmospheric water vapor using the global positioning system. Journal of Geophysical Research, 97(D14):15787.

Boese, C. M., Stern, T. A., Michailos, K., Townend, J., Boese, C. M., Stern, T. A., Michailos, K., Townend, J., and Townend, J. (2018). Implications of uppermantle seismicity for deformation in the continental collision zone beneath the 
Alpine Fault, South Island, New Zealand. New Zealand Journal of Geology and Geophysics, 0(0):1-26.

Bürgmann, R., Hilley, G., Ferretti, A., and Novali, F. (2006). Resolving vertical tectonics in the San Francisco Bay Area from permanent scatterer InSAR and GPS analysis. Geology, 34(3):221.

Bürgmann, R., Rosen, P. A., and Fielding, E. J. (2000a). Synthetic aperture radar interferometry to measure Earth's surface topography and its deformation. Annu. Rev. Earth Planet. Sci, 28:169-209.

Bürgmann, R., Schmidt, D., Nadeau, R., D’Alessio, M., Fielding, E., Manaker, D., McEvilly, T., and Murray, M. (2000b). Earthquake potential along the northern Hayward fault, California. Science, 289(5482):1178-1182.

Chaussard, E., Bürgmann, R., Fattahi, H., Johnson, C. W., Nadeau, R., Taira, T., and Johanson, I. (2015). Interseismic coupling and refined earthquake potential on the Hayward-Calaveras fault zone. Journal of Geophysical Research: Solid Earth, 120(12):8570-8590.

Chen, C. W. and Zebker, H. A. (2000). Network approaches to two-dimensional phase unwrapping: intractability and two new algorithms. Journal of the Optical Society of America A, 17(3):401.

Cochran, U. A., Clark, K. J., Howarth, J. D., Biasi, G. P., Langridge, R. M., Villamor, P., Berryman, K. R., and Vandergoes, M. J. (2017). A plate boundary earthquake record from a wetland adjacent to the Alpine fault in New Zealand refines hazard estimates. Earth and Planetary Science Letters, 464.

Demets, C., Gordon, R. G., and Argus, D. F. (2010). Geologically current plate motions. Geophys. J. Int, 181:1-80.

Doser, D. I., Webb, T. H., and Maunder, D. E. (1999). Source parameters of large historical (1918-1962) earthquakes, South Island, New Zealand. Geophysical Journal International, 139(3):769-794.

Eberhart-Phillips, D. and Reyners, M. (2001). A complex, young subduction zone imaged by three-dimensional seismic velocity, Fiordland, New Zealand. Geophysical Journal International, 146(3):731-746.

Fattahi, H. and Amelung, F. (2015). InSAR bias and uncertainty due to the systematic and stochastic tropospheric delay. Journal of Geophysical Research: Solid Earth, 120(12):8758-8773. 
Ferretti, A., Monti-guarnieri, A., Prati, C., and Rocca, F. (2007). InSAR Principles: Guidelines for SAR Interferometry Processing and Interpretation (TM-19, February 2007). Number 02.

Fialko, Y. (2006). Interseismic strain accumulation and the earthquake potential on the southern San Andreas fault system. Nature, 441(7096):968-971.

Freed, A. M. (2005). Earthquake Triggering by Static, Dynamic, and Postseismic Stress Transfer. Annual Review of Earth and Planetary Sciences.

Fry, B., Bannister, S. C., Beavan, J., Bland, L., Bradley, B. A., Cox, S. C., Cousins, J., Gale, N. H., Hancox, G., Holden, C., Jongens, R., Power, W., Prasetya, G., Reyners, M., Ristau, J., Robinson, R., Samsonov, S., and Wilson, K. (2010). The Dusky Sound EQ of 2009: A Preliminary Report. Bulletin of the New Zealand Society for Earthquake Engineering, 43(1):24-40.

Goldstein, R. M., Engelhardt, H., Kamb, B., and Frolich, R. M. (1993). Satellite radar interferometry for monitoring ice sheet motion: Application to an Antarctic ice stream. Science, 262(5139):1525-1530.

Goldstein, R. M. and Werner, C. L. (1998). Radar interferogram filtering for geophysical applications. Geophysical Research Letters, 25(21):4035-4038.

Hamling, I. J. and Hreinsdóttir, S. (2016). Reactivated afterslip induced by a large regional earthquake, Fiordland, New Zealand. Geophysical Research Letters, $43(6): 2526-2533$.

Hamling, I. J., Hreinsdóttir, S., Clark, K., Elliott, J., Liang, C., Fielding, E., Litchfield, N., Villamor, P., Wallace, L., Wright, T. J., D’Anastasio, E., Bannister, S., Burbidge, D., Denys, P., Gentle, P., Howarth, J., Mueller, C., Palmer, N., Pearson, C., Power, W., Barnes, P., Barrell, D. J. A., Van Dissen, R., Langridge, R., Little, T., Nicol, A., Pettinga, J., Rowland, J., and Stirling, M. (2017). Complex multifault rupture during the $2016 \mathrm{Mw} 7.8$ Kaikōura earthquake, New Zealand. Science, 356(6334):eaam7194.

Hanssen, R. F., Weckwerth, T. M., Zebker, H. A., and Klees, R. (1999). Highresolution water vapor mapping from interferometric radar measurements. Science, 283(5406):1297-1299.

Hooper, A., Bekaert, D., Spaans, K., and Arikan, M. (2012). Recent advances in SAR interferometry time series analysis for measuring crustal deformation. Tectonophysics, 514-517:1-13. 
Hooper, A., Zebker, H., Segall, P., and Kampes, B. (2004). A new method for measuring deformation on volcanoes and other natural terrains using InSAR persistent scatterers. Geophysical Research Letters, 31(23):1-5.

Hooper, A. J. (2008). A multi-temporal InSAR method incorporating both persistent scatterer and small baseline approaches. Geophysical Research Letters, $35(16): 1-5$.

Howarth, J. D., Cochran, U. A., Langridge, R. M., Clark, K., Fitzsimons, S. J., Berryman, K., Villamor, P., and Strong, D. T. (2018). Past large earthquakes on the Alpine Fault: paleoseismological progress and future directions. New Zealand Journal of Geology and Geophysics, 61(3):309-328.

Howarth, J. D., Fitzsimons, S. J., Norris, R. J., and Jacobsen, G. E. (2014). Lake sediments record high intensity shaking that provides insight into the location and rupture length of large earthquakes on the Alpine Fault, New Zealand. Earth and Planetary Science Letters, 403.

Howarth, J. D., Fitzsimons, S. J., Norris, R. J., Langridge, R., and Vandergoes, M. J. (2016). A 2000 yr rupture history for the Alpine fault derived from Lake Ellery, South Island, New Zealand. Bulletin of the Geological Society of America, 128(3-4):627-643.

Jónsson, S., Zebker, H., Segall, P., and Amelung, F. (2002). Fault slip distribution of the 1999 Mw 7.1 Hector Mine, California, earthquake, estimated from satellite radar and GPS measurements. Bulletin of the Seismological Society of America, 92(41):1377-1389.

Lamb, S. (2015). Kinematics to dynamics in the New Zealand Plate boundary zone: implications for the strength of the lithosphere. Geophysical Journal International, 201:552-573.

Langridge, R. M., Ries, W. F., Litchfield, N. J., Villamor, P., Van Dissen, R. J., Barrell, D. J., Rattenbury, M. S., Heron, D. W., Haubrock, S., Townsend, D. B., Lee, J. M., Berryman, K. R., Nicol, A., Cox, S. C., and Stirling, M. W. (2016). The New Zealand Active Faults Database. New Zealand Journal of Geology and Geophysics, 59(1).

Langridge, R. M., Villamor, P., Basili, R., Almond, P., Martinez-Diaz, J. J., and Canora, C. (2010). Revised slip rates for the Alpine fault at Inchbonnie: Implications for plate boundary kinematics of South Island, New Zealand. Lithosphere, 2(3):139-152. 
Liang, C. and Fielding, E. J. (2017). Interferometry with ALOS-2 full-aperture ScanSAR data. IEEE Transactions on Geoscience and Remote Sensing, 55(5):2739-2750.

Lyons, S. and Sandwell, D. (2003). Fault creep along the southern San Andreas from interferometric synthetic aperture radar, permanent scatterers, and stacking. Journal of Geophysical Research: Solid Earth, 108(B1).

Massonnet, D. and Feigl, K. L. (1998). Radar interferometry and its application to changes in the earth's surface. Reviews of Geophysics, 36(4):441-500.

Massonnet, D., Rossi, M., Carmona, C., Adragna, F., Peltzer, G., Feigl, K., and Rabaute, T. (1993). The displacement field of the Landers earthquake mapped by radar interferometry. Nature, 364(6433):138-142.

Mortimer, N., Campbell, H. J., Tulloch, A. J., King, P. R., Stagpoole, V. M., Wood, R. A., Rattenbury, M. S., Sutherland, R., Adams, C. J., Collot, J., and Seton, M. (2017). Zealandia: Earth's hidden continent. GSA Today, 27(3):27-35.

Norris, R. J. and Cooper, A. F. (2001). Late Quaternary slip rates and slip partitioning on the Alpine Fault, New Zealand. Journal of Structural Geology, 23(2-3):507-520.

Okada, Y. (1985). Surface deformation due to shear and tensile faults in a half-space. Bulletin of the Seismological Society of America, 75(4):1135-1154.

Osmanoğlu, B., Sunar, F., Wdowinski, S., and Cabral-Cano, E. (2016). Time series analysis of InSAR data: Methods and trends. ISPRS Journal of Photogrammetry and Remote Sensing, 115:90-102.

Peng, Z., Fry, B., Chao, K., Yao, D., Meng, X., and Jolly, A. (2018). Remote triggering of microearthquakes and tremor in New Zealand following the 2016 M7.8 Kaikoura earthquake. Seismological Society of America, 108(3B):17841793.

Petersen, T., Ristau, J., Beavan, J., Denys, P., Denham, M., Field, B., Françoisholden, C., Palmer, N., Reyners, M., and Geonet, T. (2009). The Mw 6.7 George Sound earthquake of October 15, 2007: response and preliminary results. Bulletin of the New Zealand Society for Earthquake Engineering, 42(2):129-141.

Price, E. J. and Sandwell, D. T. (1998). Small-scale deformations associated with the 1992 Landers, California, earthquake mapped by synthetic aperture radar interferometry phase gradients. Journal of Geophysical Research: Solid Earth, 103(B11):27001-27016. 
Pritchard, M. E., Simons, M., Rosen, P. A., Hensley, S., and Webb, F. H. (2002). Co-seismic slip from the 1995 July $30 \mathrm{Mw}=8.1$ Antofagasta, Chile, earthquake as constrained by InSAR and GPS observations. Geophysical Journal International, 150(2):362-376.

Reyners, M., Eberhart-Phillips, D., Upton, P., and Gubbins, D. (2017). Threedimensional imaging of impact of a large igneous province with a subduction zone. Earth and Planetary Science Letters, 460:143-151.

Reyners, M., Gledhill, K., and Waters, D. (1991). Tearing of the subducted Australian Plate during the Te Anau, New Zealand, earthquake of 1988 June 3. Geophysical Journal International, 104(1):105-115.

Reyners, M., McGinty, P., Cox, S., Turnbull, I., O’Neill, T., Gledhill, K., Hancox, G., Beavan, J., Matheson, D., McVerry, G., Cousins, J., Zhao, J., Cowan, H., Caldwell, G., and Bennie, S. (2003). The Mw 7.2 Fiordland earthquake of August 21, 2003: Background and preliminary results. Bulletin of the New Zealand Society for Earthquake Engineering, 36(4):233-248.

Reyners, M., Robinson, R., Pancha, A., and McGinty, P. (2002). Stresses and strains in a twisted subduction zone-Fiordland, New Zealand. Geophys. J. Int, 148:637-648.

Reyners, M. and Webb, T. (2002). Large earthquakes near doubtful sound, New Zealand, 1989-93. New Zealand Journal of Geology and Geophysics, 45(1):109 120.

Ristau, J., Harte, D., and Salichon, J. (2016). A revised local magnitude (ML) scale for New Zealand earthquakes. Bulletin of the Seismological Society of America, 106(2):398-407.

Rogers, G. and Dragert, H. (2003). Episodic tremor and slip on the Cascadia subduction zone: the chatter of silent slip. Science (New York, N.Y.), 300(5627):19423 .

Romanet, P. and Ide, S. (2019). Ambient tectonic tremors in Manawatu, Cape Turnagain, Marlborough, and Puysegur, New Zealand. Earth, Planets and Space, 71(1):59.

Rosen, P., Hensley, S., Joughin, I., Li, F., Madsen, S., Rodriguez, E., and Goldstein, R. (2000). Synthetic aperture radar interferometry. Proceedings of the IEEE, 88(3):333-382. 
Rosen, P. A., Hensley, S., Shaffer, S., Veilleux, L., Chakraborty, M., Misra, T., Bhan, R., Raju Sagi, V., and Satish, R. (2015). The NASA-ISRO SAR mission - An international space partnership for science and societal benefit. In IEEE National Radar Conference - Proceedings, volume 2015-June, pages 1610-1613. IEEE.

Rosenqvist, A., Shimada, M., Ito, N., and Watanabe, M. (2007). ALOS PALSAR: A Pathfinder Mission for Global-Scale Monitoring of the Environment. IEEE Transactions on Geoscience and Remote Sensing, 45(11):3307-3316.

Sandwell, A., Mellors, D., Tong, R., Sandwell, D., Mellors, R., Tong, X., Wei, M., and Wessel, P. (2011a). GMTSAR: An InSAR Processing System Based on Generic Mapping Tools Scripps Institution of Oceanography Technical Report GMTSAR: An InSAR Processing System Based on Generic Mapping Tools. Technical report, UC San Diego: Scripps Institution of Oceanography, San Diego.

Sandwell, D., Mellors, R., Tong, X., Wei, M., and Wessel, P. (2011b). Open radar interferometry software for mapping surface Deformation. Eos, Transactions American Geophysical Union, 92(28):234-234.

Schmidt, D. A. and Bürgmann, R. (2003). Time-dependent land uplift and subsidence in the Santa Clara valley, California, from a large interferometric synthetic aperture radar data set. Journal of Geophysical Research: Solid Earth, 108(B9).

Shanker, A. P. and Zebker, H. A. (2009). Sparse two-dimensional phase unwrapping using regular grid methods. IEEE Geoscience and Remote Sensing Letters, 6(3):519-522.

Simons, M., Fialko, Y., and Rivera, L. (2002). Earthquake as Inferred from InSAR and GPS Observations. Bulletin of the Seismological Society of America, 92(41):1390-1402.

Simons, M. and Rosen, P. A. (2015). Interferometric Synthetic Aperture Radar Geodesy. In Treatise on Geophysics, volume 3, pages 339-385. Oxford: Elsevier, 2 edition.

Stern, T., Molnar, P., Okaya, D., and Eberhart-Phillips, D. (2000). Teleseismic $\mathrm{P}$ wave delays and modes of shortening the mantle lithosphere beneath South Island, New Zealand. Journal of Geophysical Research: Solid Earth, 105(B9):21615-21631. 
Stirling, M. W., Litchfield, N. J., Villamor, P., Van Dissen, R. J., Nicol, A., Pettinga, J., Barnes, P., Langridge, R. M., Little, T., Barrell, D. J., Mountjoy, J., Ries, W. F., Rowland, J., Fenton, C., Hamling, I., Asher, C., Barrier, A., Benson, A., Bischoff, A., Borella, J., Carne, R., Cochran, U. A., Cockroft, M., Cox, S. C., Duke, G., Fenton, F., Gasston, C., Grimshaw, C., Hale, D., Hall, B., Hao, K. X., Hatem, A., Hemphill-Haley, M., Heron, D. W., Howarth, J., Juniper, Z., Kane, T., Kearse, J., Khajavi, N., Lamarche, G., Lawson, S., Lukovic, B., Madugo, C., Manousakis, I., McColl, S., Noble, D., Pedley, K., Sauer, K., Stah, T., Strong, D. T., Townsend, D. B., Toy, V., Villeneuve, M., Wandres, A., Williams, J., Woelz, S., and Zinke, R. (2017). The Mw7.8 2016 Kaikōura earthquake: Surface fault rupture and seismic hazard context. Bulletin of the New Zealand Society for Earthquake Engineering, 50(2).

Sutherland, R. (1995). The Australian-Pacific boundary and Cenozoic plate motions in the SW Pacific. Tectonics, 14(4):819-831.

Sutherland, R., Davey, F., and Beavan, J. (2000). Plate boundary deformation in South Island, New Zealand, is related to inherited lithospheric structure. Earth and Planetary Science Letters, 177(3-4):141-151.

Sutherland, R., Eberhart-Phillips, D., Harris, R. A., Stern, T., Beavan, J., Ellis, S., Henrys, S., Cox, S., Norris, R. J., Berryman, K. R., Townend, J., Bannister, S., Pettinga, J., Leitner, B., Wallace, L., Little, T. A., Cooper, A. F., Yetton, M., and Stirling, M. (2007). Do great earthquakes occur on the Alpine Fault in central South Island, New Zealand? Geophysical Monograph Series, 175:235251 .

Sutherland, R., Gurnis, M., Kamp, P. J., and House, M. A. (2009). Regional exhumation history of brittle crust during subduction initiation, Fiordland, southwest New Zealand, and implications for thermochronologic sampling and analysis strategies. Geosphere, 5(5):409-425.

Torres, R., Snoeij, P., Geudtner, D., Bibby, D., Davidson, M., Attema, E., Potin, P., Rommen, B., Floury, N., Brown, M., Traver, I. N., Deghaye, P., Duesmann, B., Rosich, B., Miranda, N., Bruno, C., L'Abbate, M., Croci, R., Pietropaolo, A., Huchler, M., and Rostan, F. (2012). GMES Sentinel-1 mission. Remote Sensing of Environment, 120:9-24.

Tymofyeyeva, E. and Fialko, Y. (2015). Mitigation of atmospheric phase delays in InSAR data, with application to the eastern California shear zone. Journal of Geophysical Research: Solid Earth, 120(8):5952-5963. 
Wallace, L. M., Hreinsdóttir, S., Ellis, S., Hamling, I., D'Anastasio, E., and Denys, P. (2018). Triggered Slow Slip and Afterslip on the Southern Hikurangi Subduction Zone Following the Kaikōura Earthquake. Geophysical Research Letters, pages 4710-4718.

Warren-Smith, E., Lamb, S., Seward, D., Smith, E., Herman, F., and Stern, T. (2016). Thermochronological evidence of a low-angle, mid-crustal detachment plane beneath the central South Island, New Zealand. Geochemistry Geophysics Geosystems, 17:1312-1338.

Warren-Smith, E., Lamb, S., Stern, T. A., and Smith, E. (2017). Microseismicity in Southern South Island, New Zealand: Implications for the Mechanism of Crustal Deformation Adjacent to a Major Continental Transform. Journal of Geophysical Research: Solid Earth, 122(11):9208-9227.

Wei, M. and Sandwell, D. T. (2010). Decorrelation of L-band and C-band interferometry over vegetated areas in California. IEEE Transactions on Geoscience and Remote Sensing, 48(7):2942-2952.

Wessel, P. and Smith, W. (2013). Generic Mapping Tools: Inproved version released. EOS Trans. AGU, 94(45):409-10.

Zebker, H. A., Rosen, P. A., Goldstein, R. M., Gabriel, A., and Werner, C. L. (1994). On the derivation of coseismic displacement fields using differential radar interferometry: The Landers earthquake. Journal of Geophysical Research: Solid Earth, 99(B10):19617-19634. 
Appendices 



\begin{tabular}{|c|c|c|c|c|c|c|}
\hline $\mathrm{nx}$ & $\begin{array}{l}\text { x_search } \\
\text { ny / y_search }\end{array}$ & $\begin{array}{l}16 \\
32\end{array}$ & $\begin{array}{l}32 \\
64\end{array}$ & $\begin{array}{l}64 \\
128\end{array}$ & $\begin{array}{l}128 \\
256\end{array}$ & $\begin{array}{l}256 \\
512\end{array}$ \\
\hline 5 & 12 & & & & $\begin{array}{l}47.26 \\
40.47 \\
60\end{array}$ & \\
\hline 10 & 25 & & & & $\begin{array}{l}45.06 \\
185.76 \\
250\end{array}$ & \\
\hline 20 & 50 & $\begin{array}{l}34.11 \\
78.97 \\
1000\end{array}$ & $\begin{array}{l}37.34 \\
117.93 \\
1000\end{array}$ & $\begin{array}{l}40.97 \\
272.63 \\
1000\end{array}$ & $\begin{array}{l}44.53 \\
730.52 \\
1000\end{array}$ & $\begin{array}{l}47.71 \\
2,417.38 \\
1000\end{array}$ \\
\hline 30 & 75 & & & & $\begin{array}{l}43.82 \\
1,656.83 \\
2250\end{array}$ & \\
\hline 40 & 100 & & & & $\begin{array}{l}43.72 \\
2,916.81 \\
4000\end{array}$ & $\begin{array}{l}\text { average SNR } \\
\text { processing time }(\mathrm{s}) \\
\text { total\# of CC locations }\end{array}$ \\
\hline
\end{tabular}

Table 1: Results of cross correlation (CC) testing between 21 Dec 2006 and 05 Feb 2007 (track 348, frame 6260). The template size (number of pixels in $\mathrm{x}(\mathrm{nx})$ and $\mathrm{y}$ (yn) directions) and total number of CC (specified by total number in $\mathrm{x}$ ( $\mathrm{x}$ _search) and in $\mathrm{y}$ (y_search) directions) have been varied from the GMTSAR default values. As the template size increases, the SNR increases. For each test the top value is the average SNR, the middle value the processing time in seconds and the bottom value is the total number of CC completed
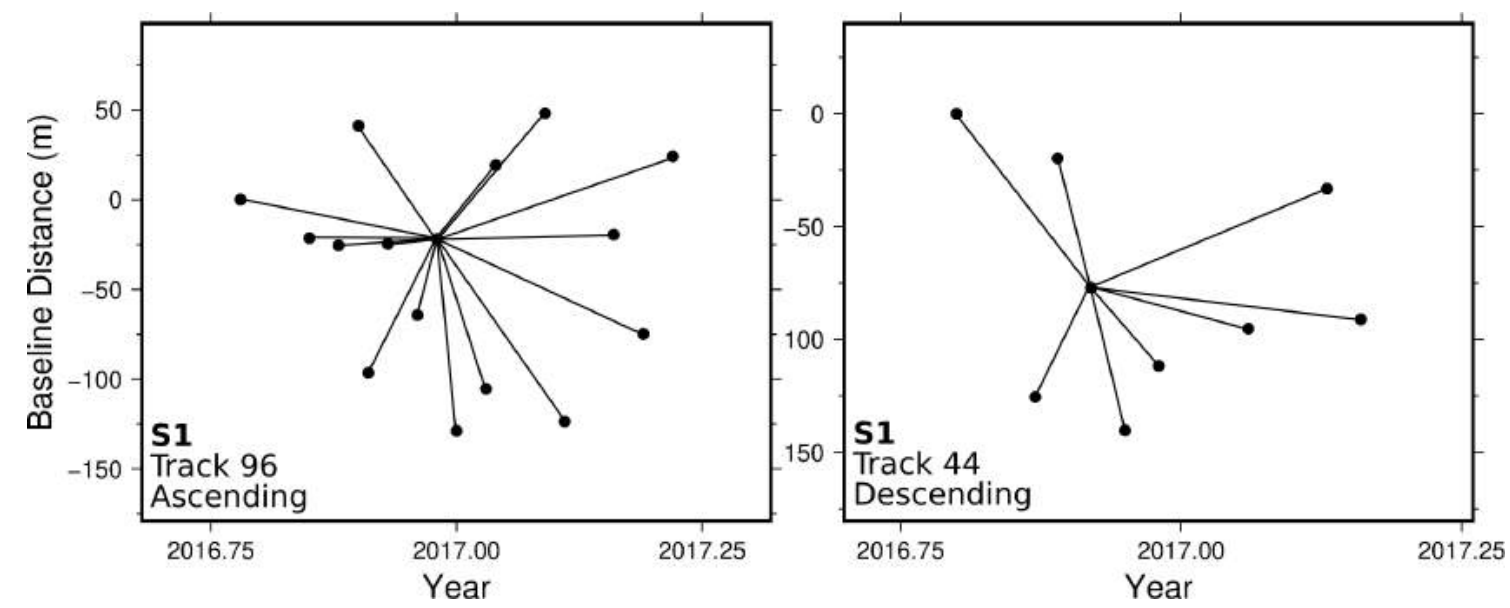

Figure A1: Baseline distance vs. time plot of SAR acquisition alignment. As all acquisitions were within $200 \mathrm{~m}$ of each other all slave images were aligned to a single master. 


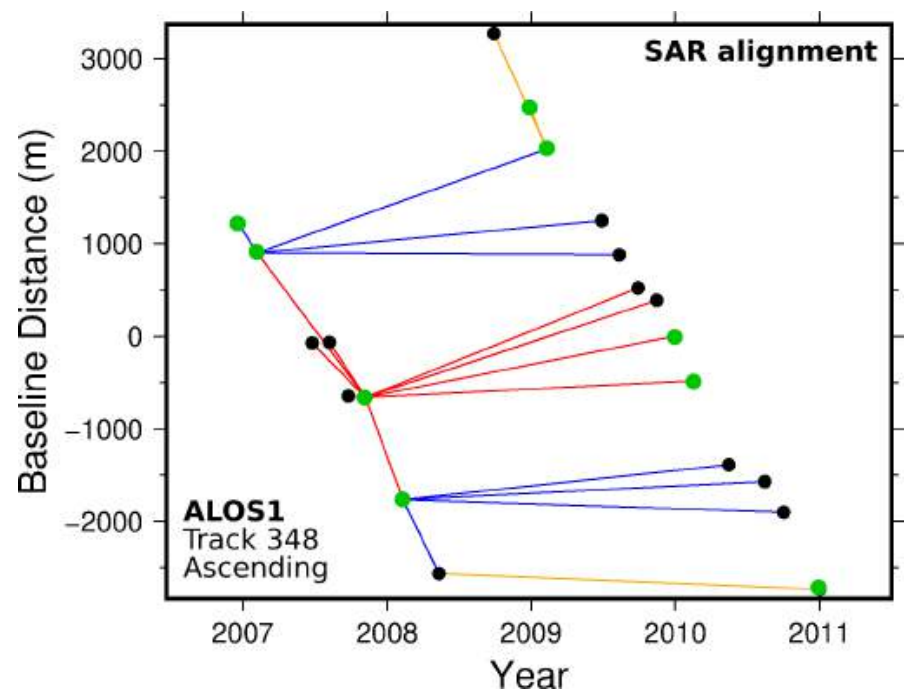

Figure A2: Baseline distance vs. time plot of alternative alignment of SAR images. This alternative alignment arrangement produced a very similar result to that of Figure 3.6. Green dots indicate double polarisation acquisitions and black dots are single polarisation. 

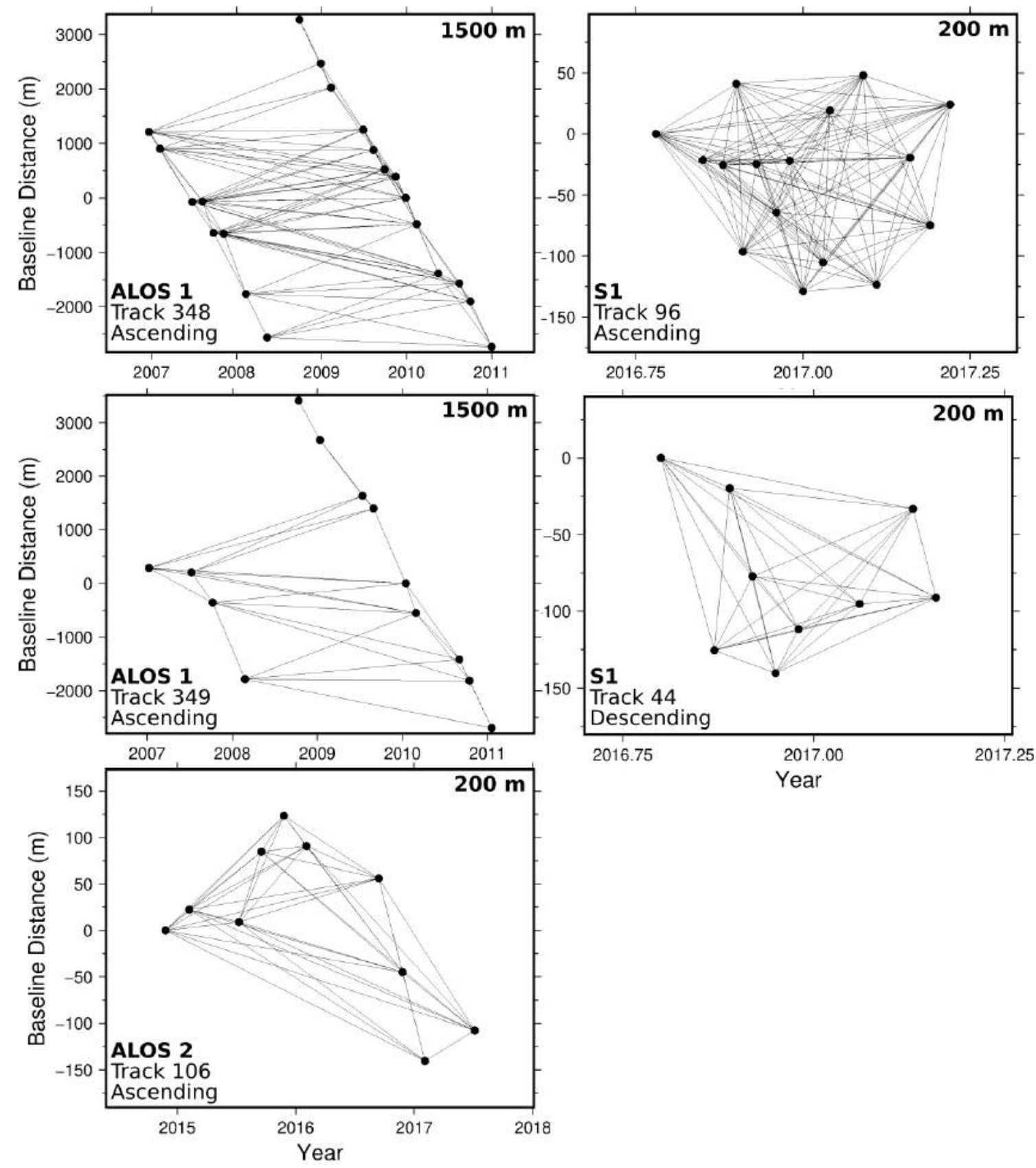

Figure A3: Plot of final interferogram networks. ALOS1 tracks have a maximum baseline separation of $1500 \mathrm{~m}$, while ALOS2 and Sentinel (S1) have a maximum separation of $200 \mathrm{~m}$. 

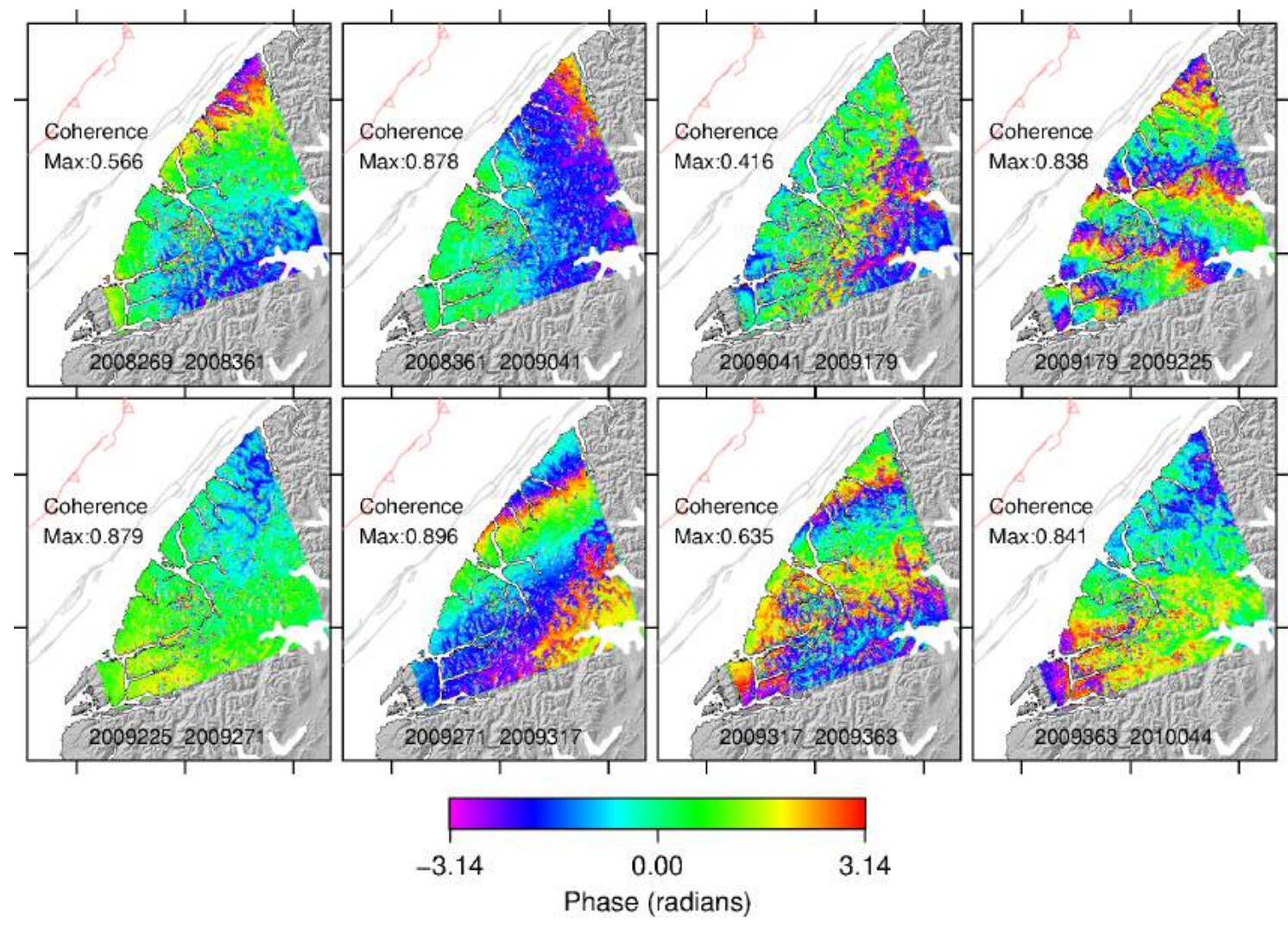

Figure A4: Preliminary investigation. ALOS1 wrapped phase from track 348, frame 6260 for the period 25 September 2008 to 13 February 2010.
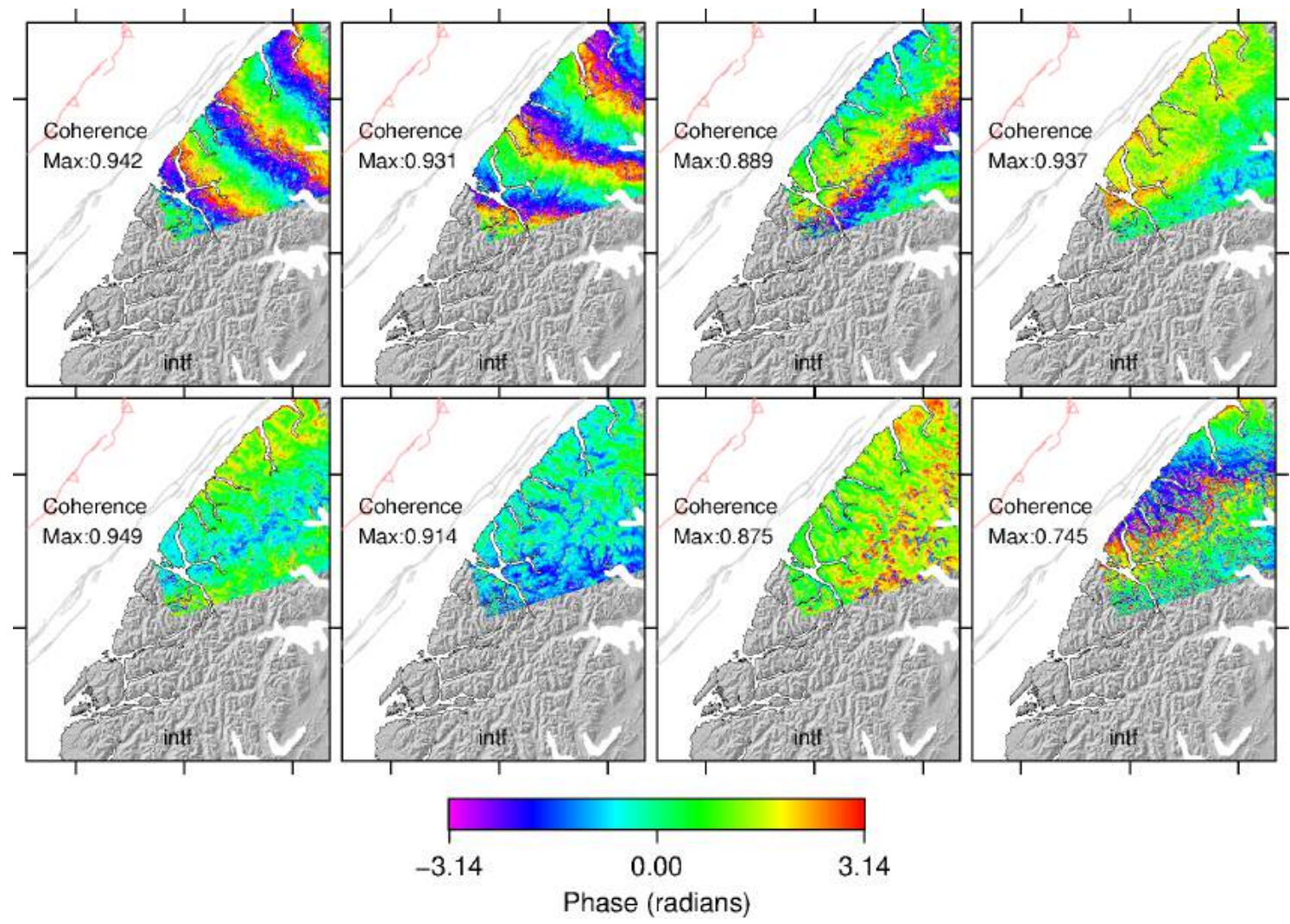

Figure A5: Preliminary investigation - ALOS2 Wrapped phase from track 105, frame 6270 for the period 16 September 2015 to 8 November 2017 


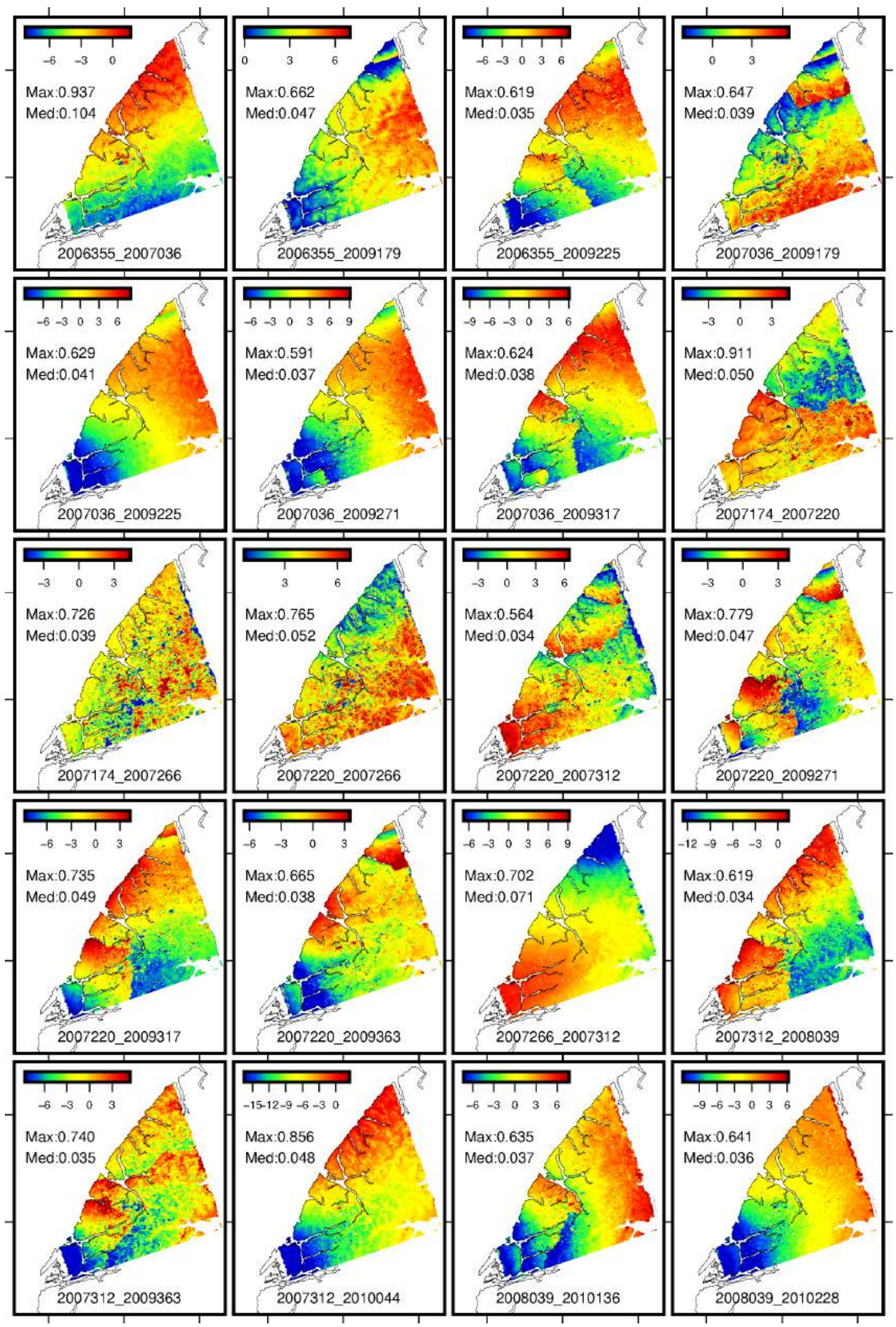

Figure A6: Interferograms unwrapped with no interpolation, processed with a $400 \mathrm{~m}$ Gaussian Filter and a coherence threshold of 0.12. Unwrapping errors observed as steps in the data are present in 17 of the 20 interferograms presented. The maximum and median coherence value for each interferogram is presented. Images with a lower median coherence include more unwrapping errors. 


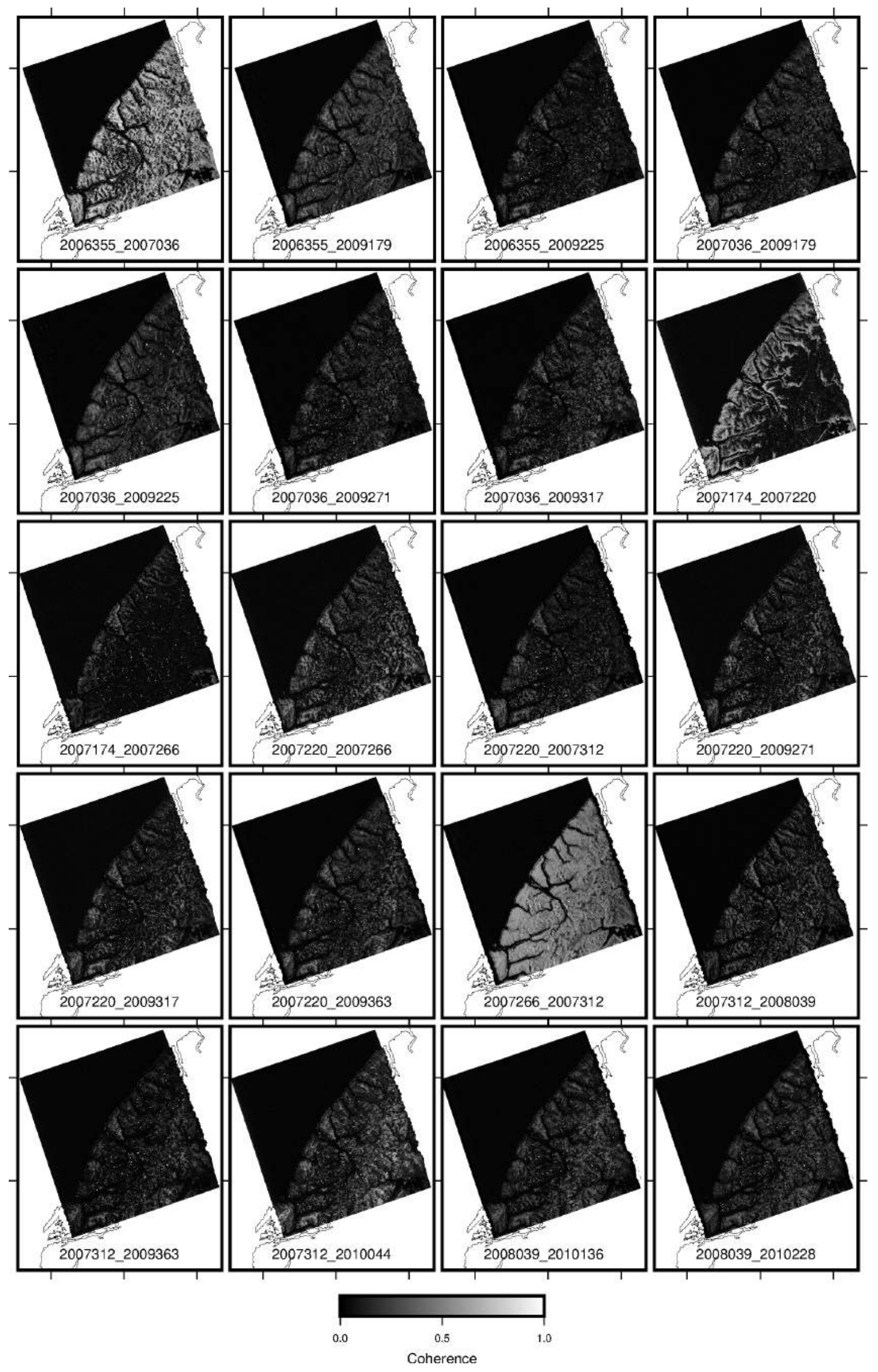

Figure A7: Coherence plots for the interferograms presented in Figure A6. Images with short temporal separations had more uniform coherence. 

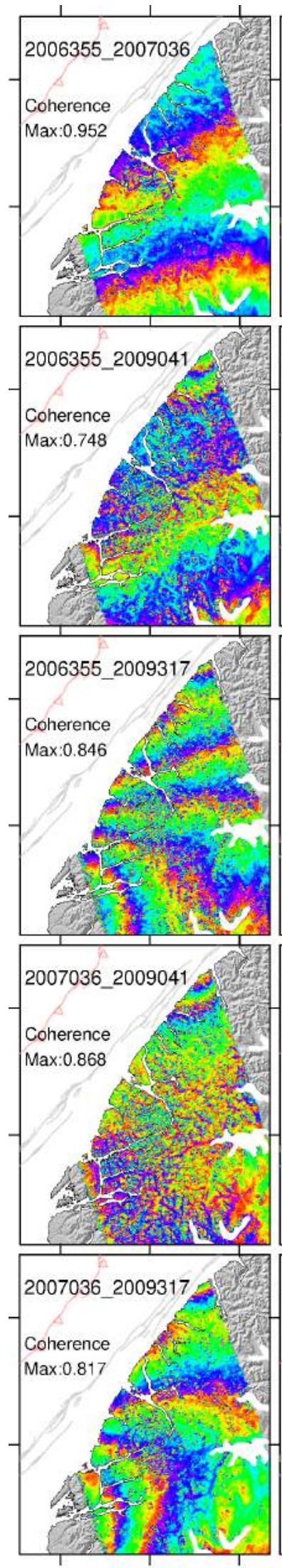
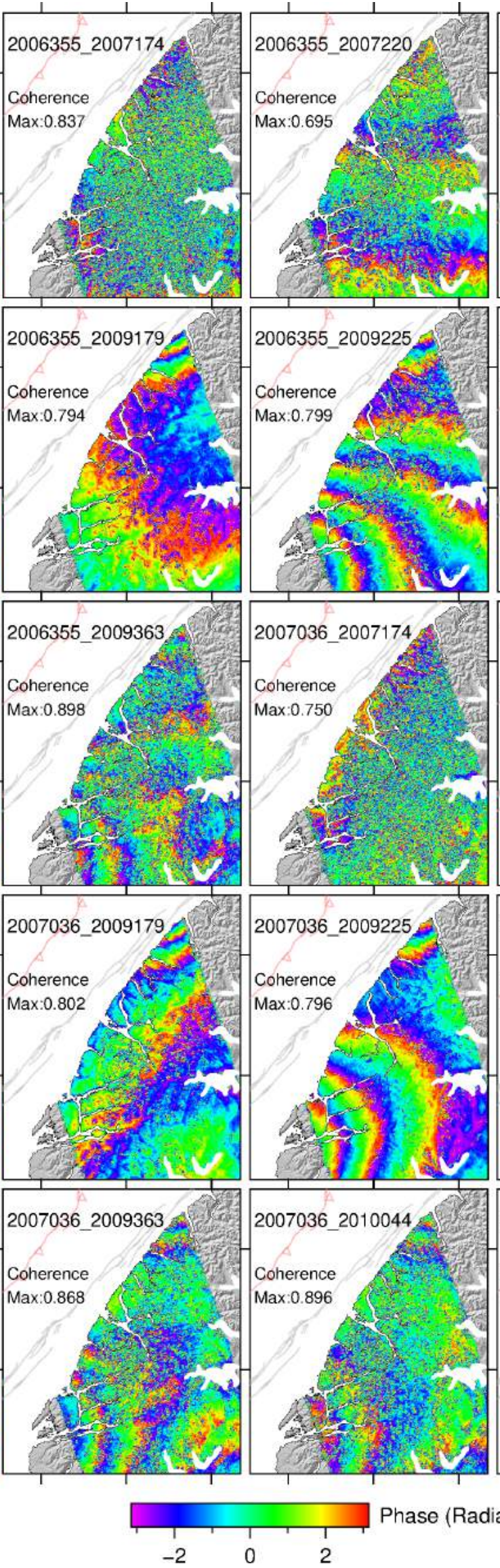

Phase (Radians)
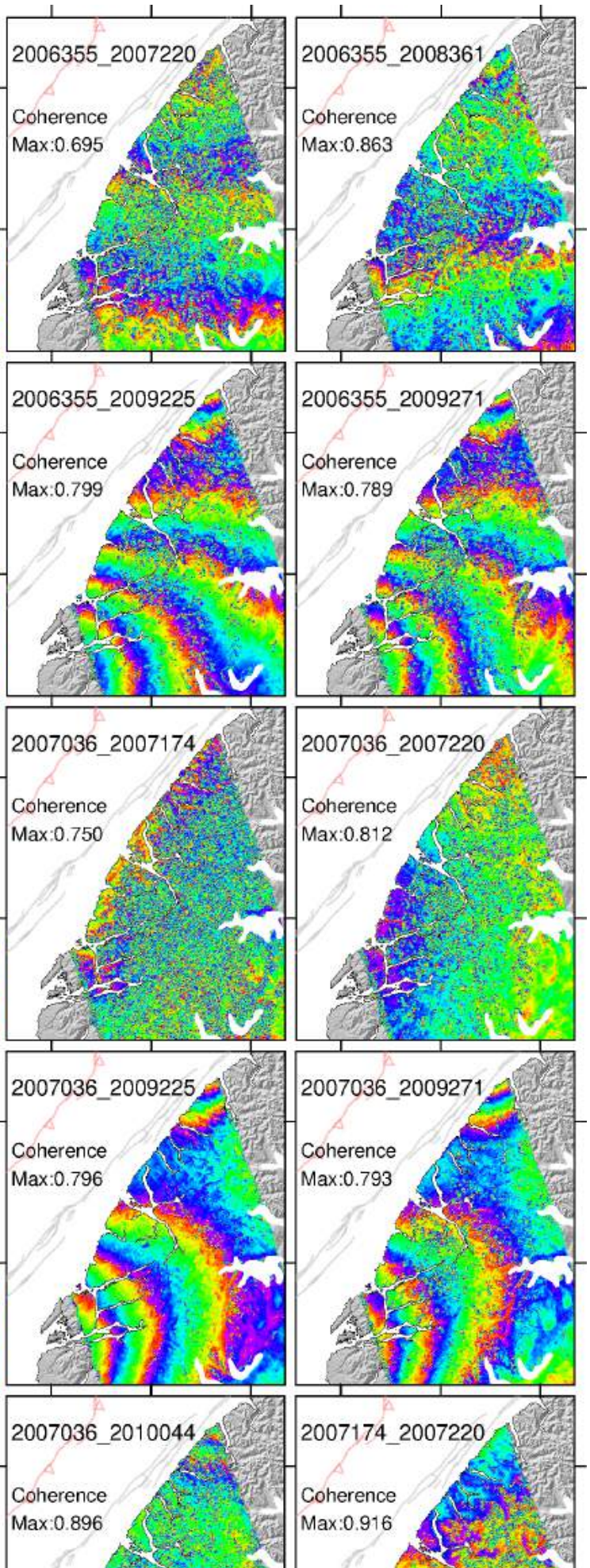

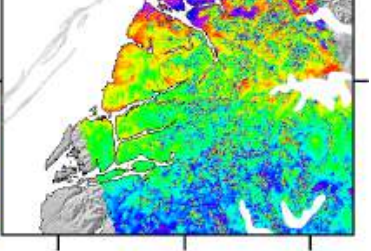

\section{ans)}

Figure A8: Final Interferograms - Wrapped Phase 1 

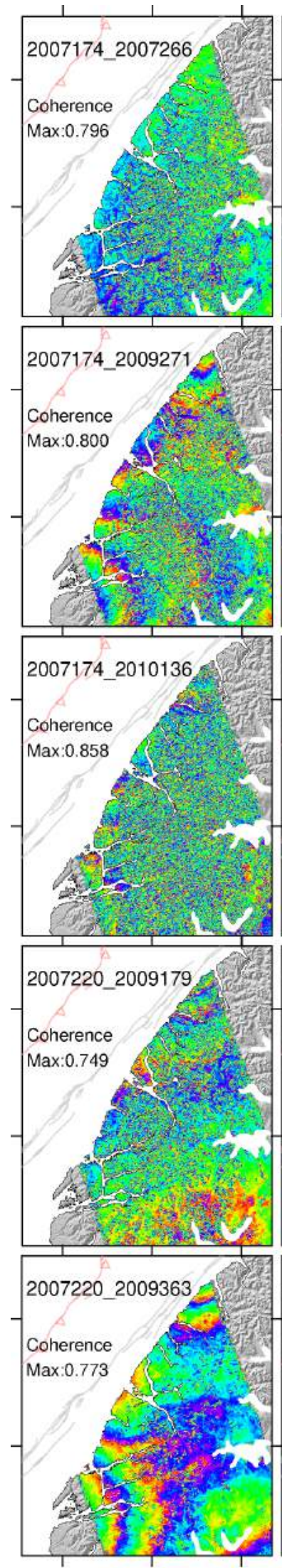
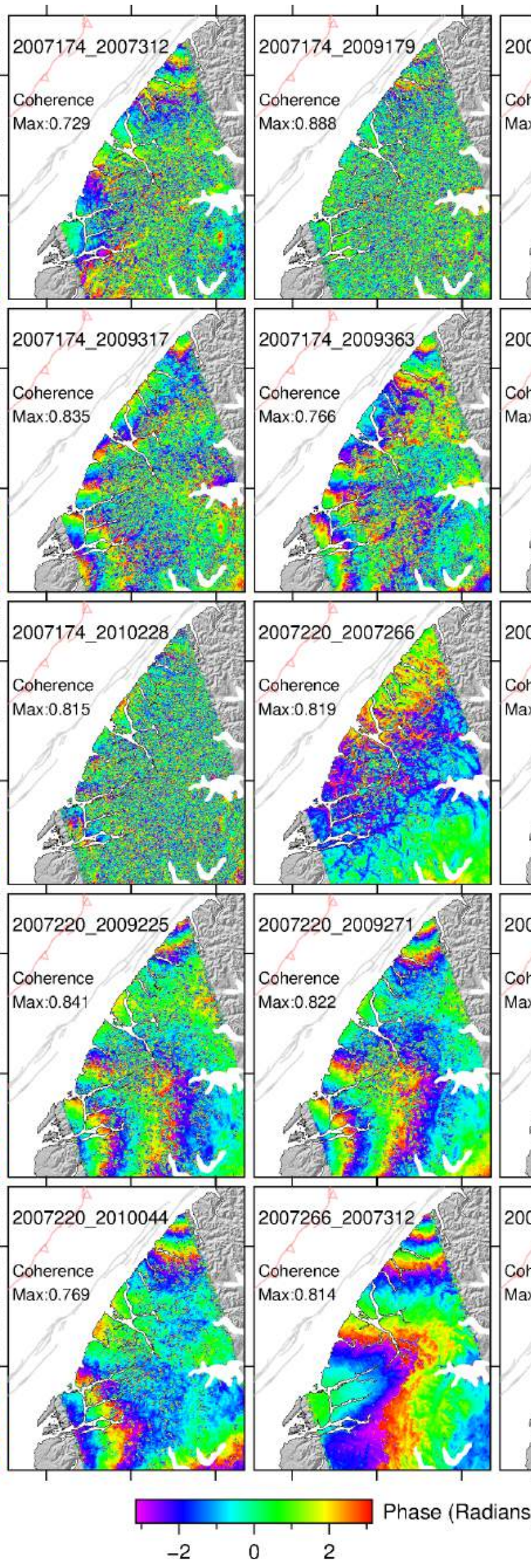
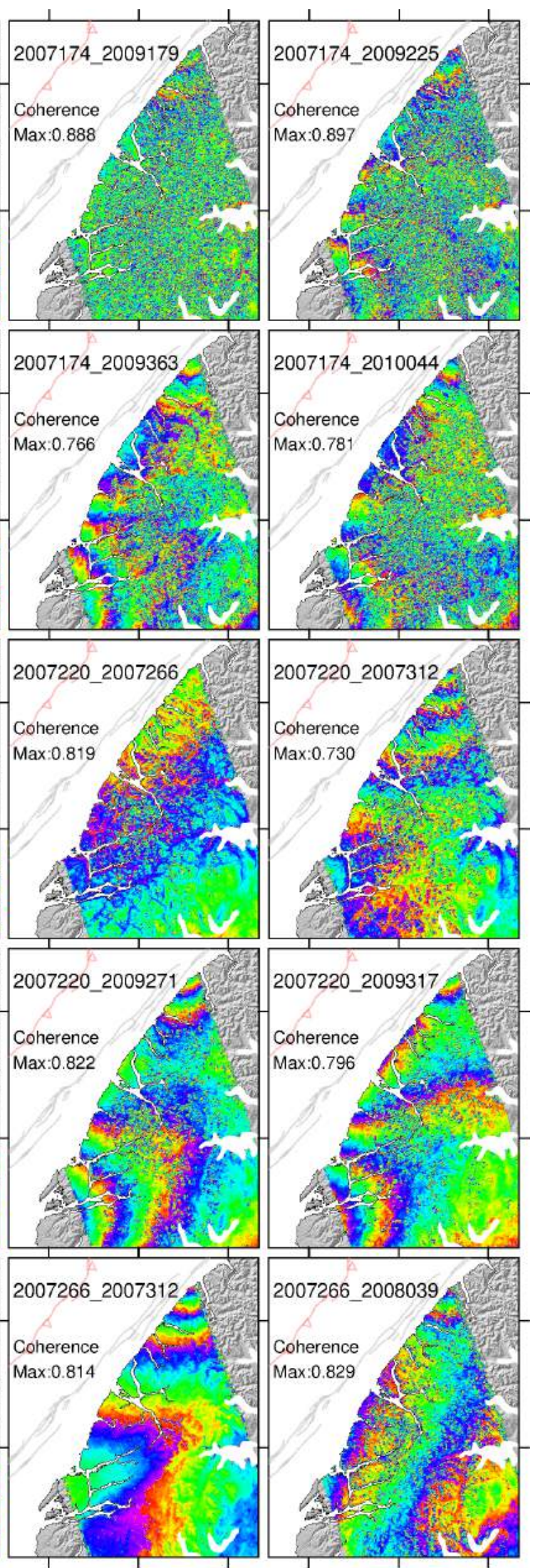

ns)

Figure A9: Final Interferograms - Wrapped Phase 2 

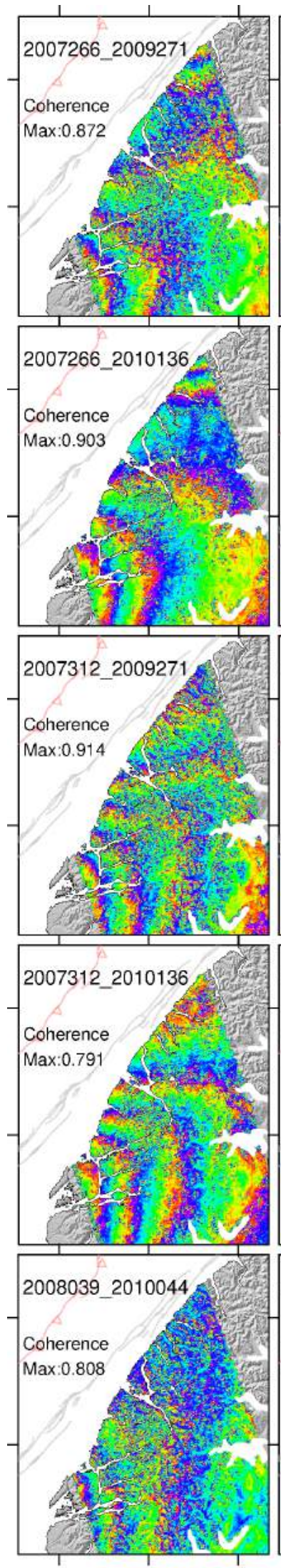
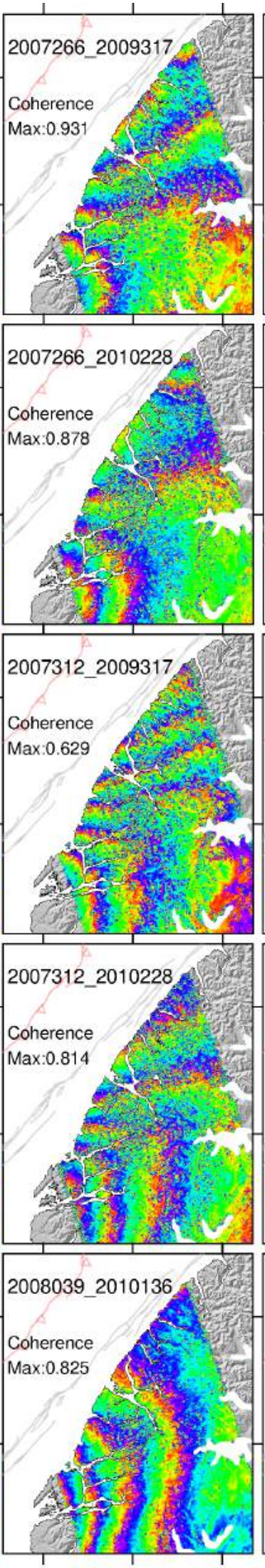
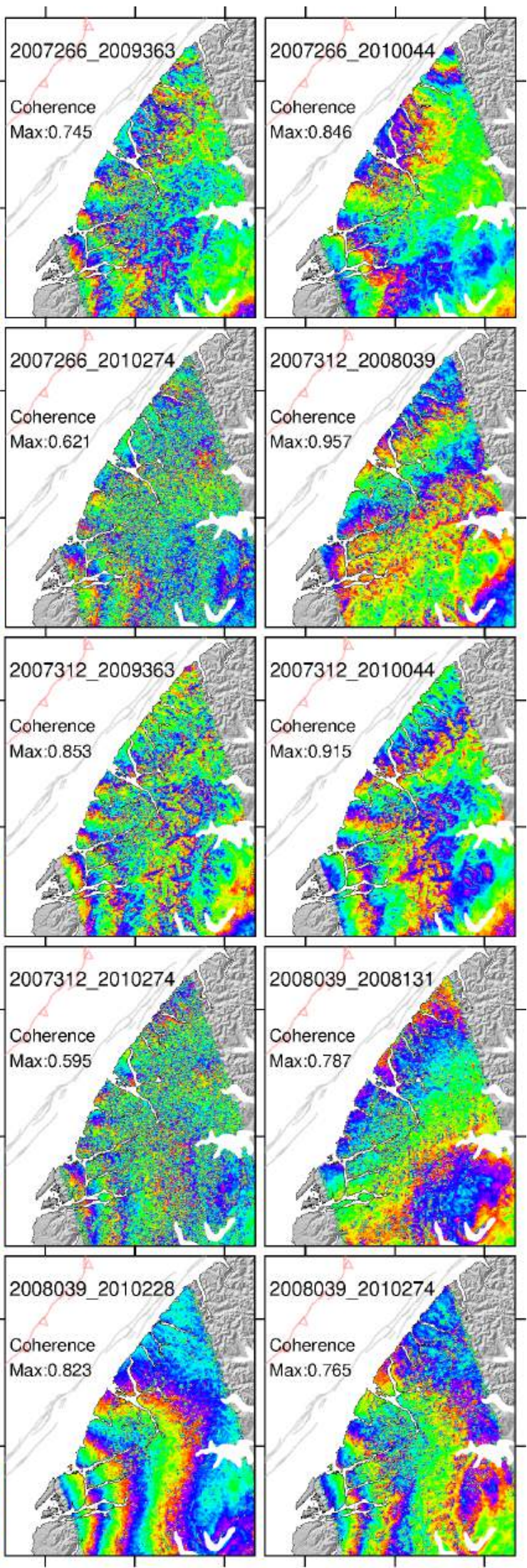

Phase (Radians)

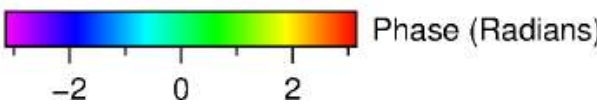

Figure A10: Final Interferograms - Wrapped Phase 3 

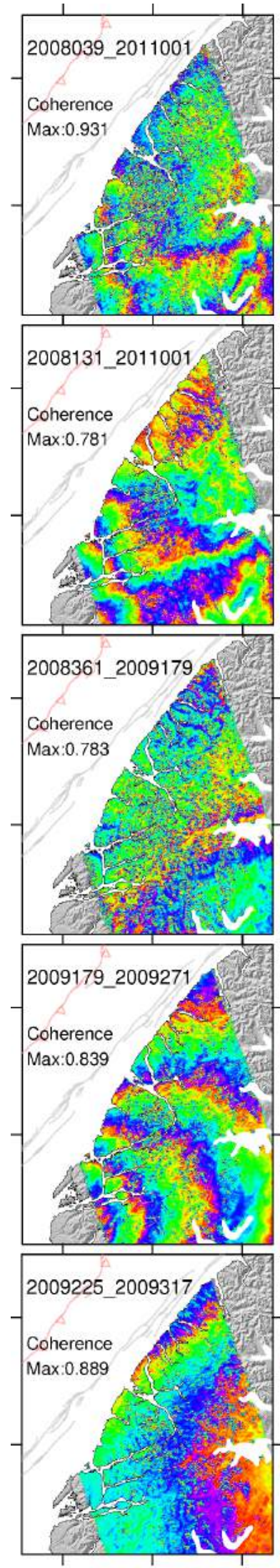
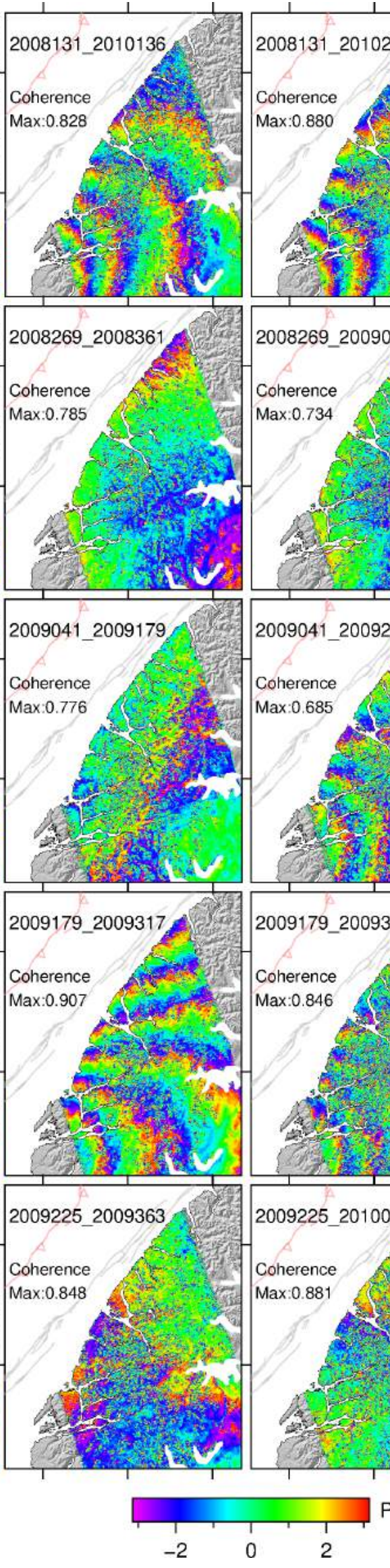

Phase (Radians)
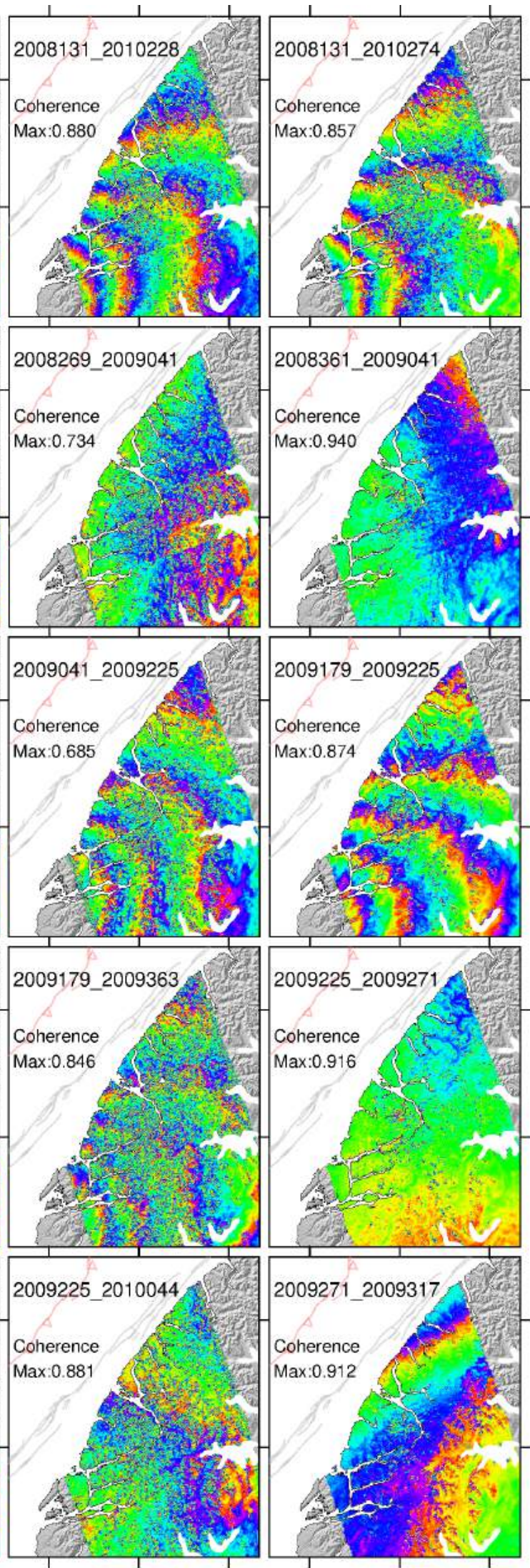

n)

Figure A11: Final Interferograms - Wrapped Phase 4 

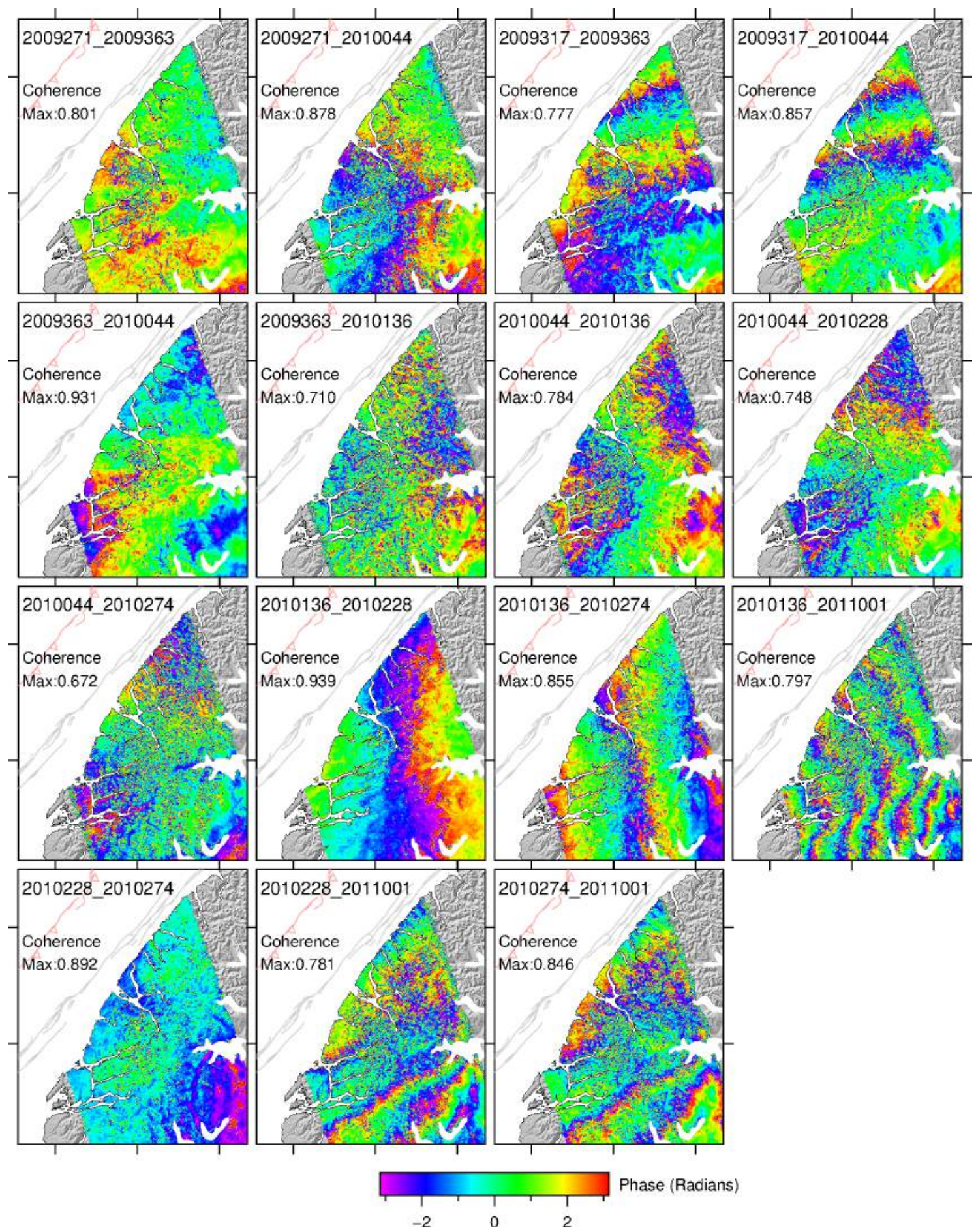

$\operatorname{Max}: 0.846$

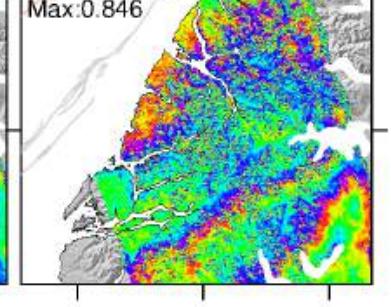

Figure A12: Final Interferograms - Wrapped Phase 5 

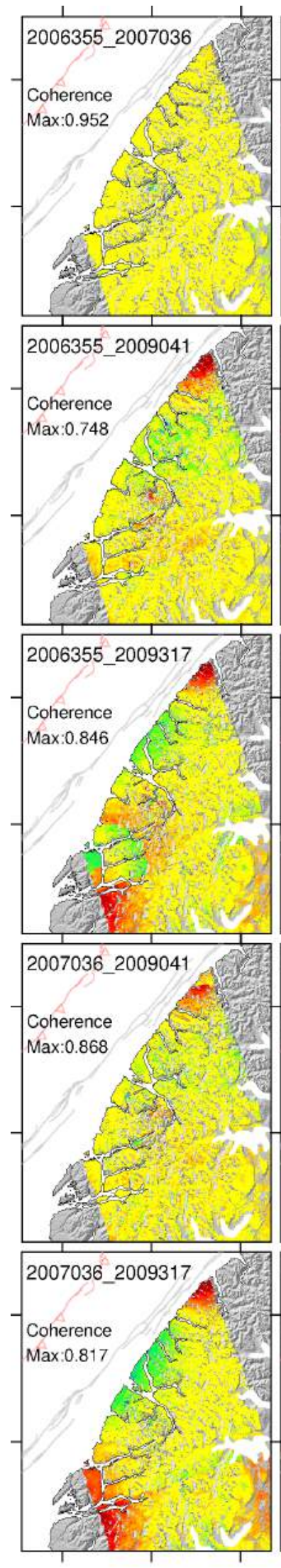
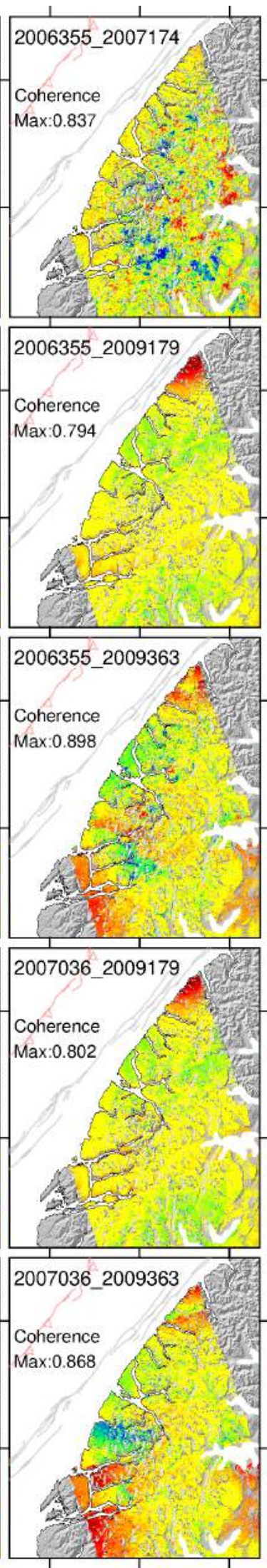
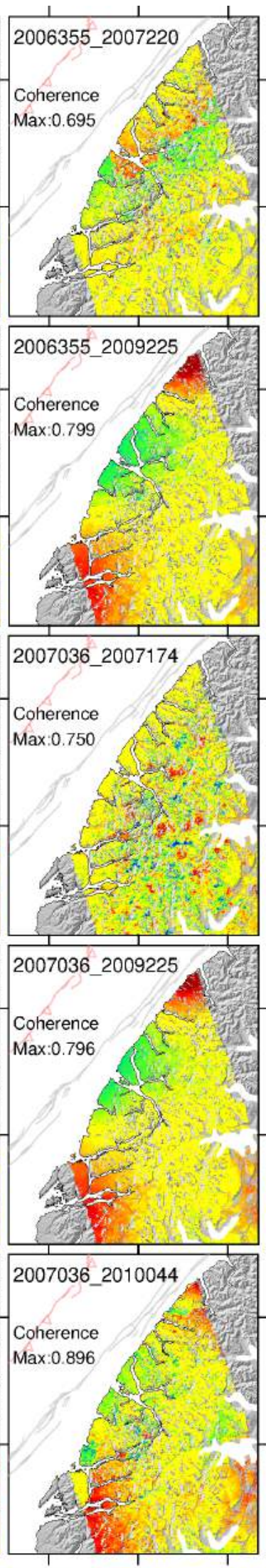

Phase (Radians)
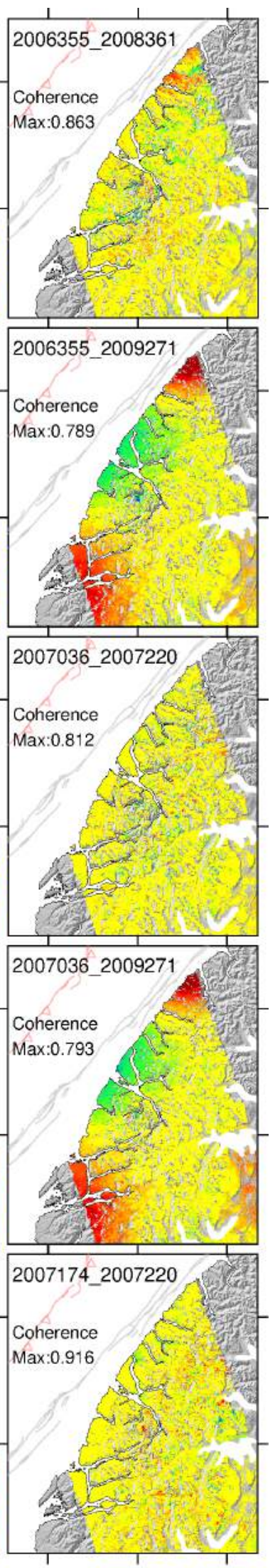

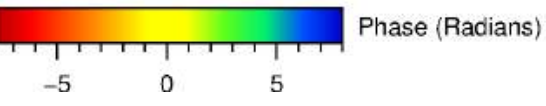

Figure A13: Final Interferograms - Unwrapped Phase 1 

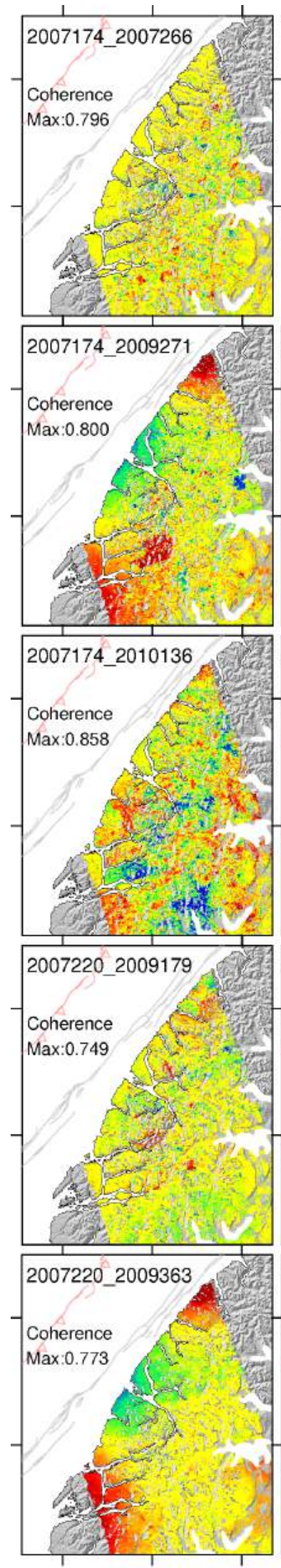
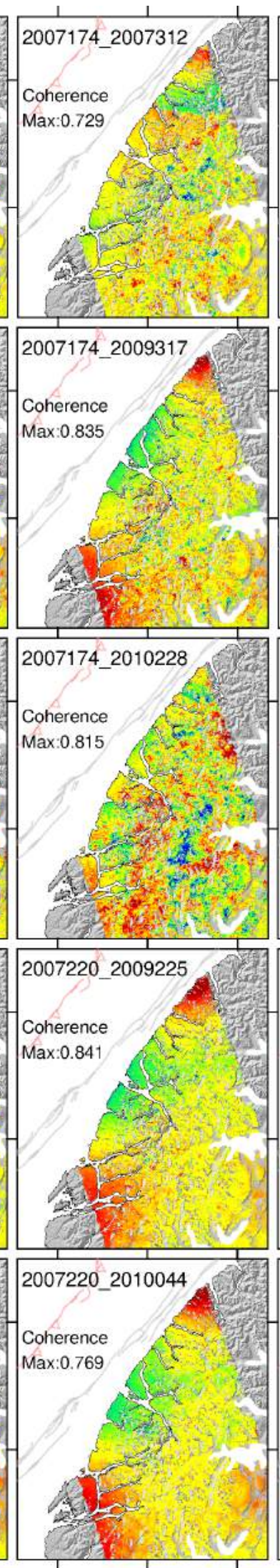
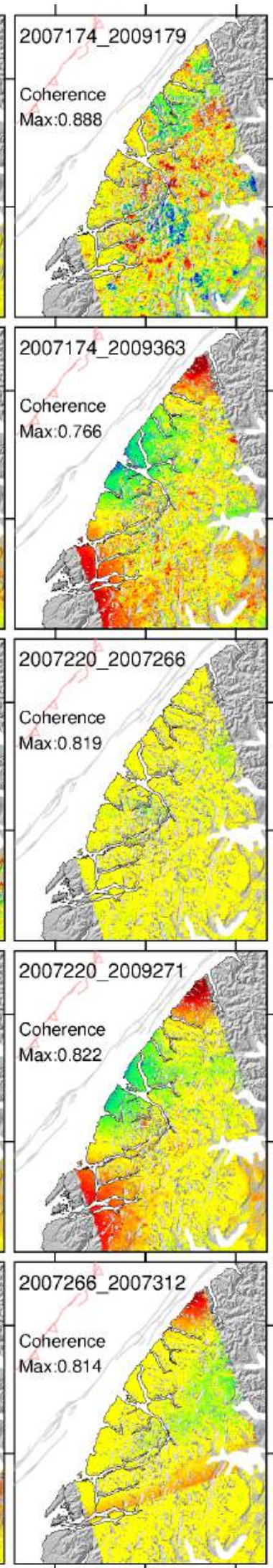

Phase (Radians)
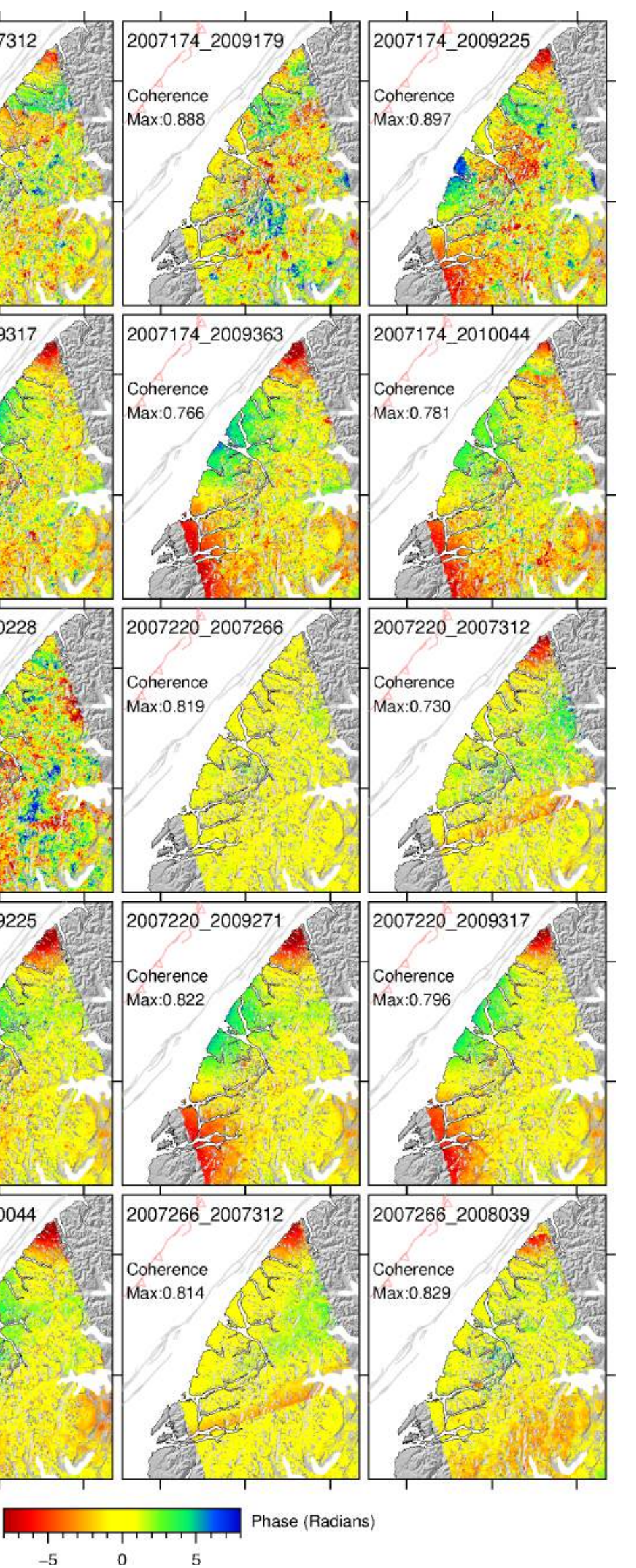

Figure A14: Final Interferograms - Unwrapped Phase 2 

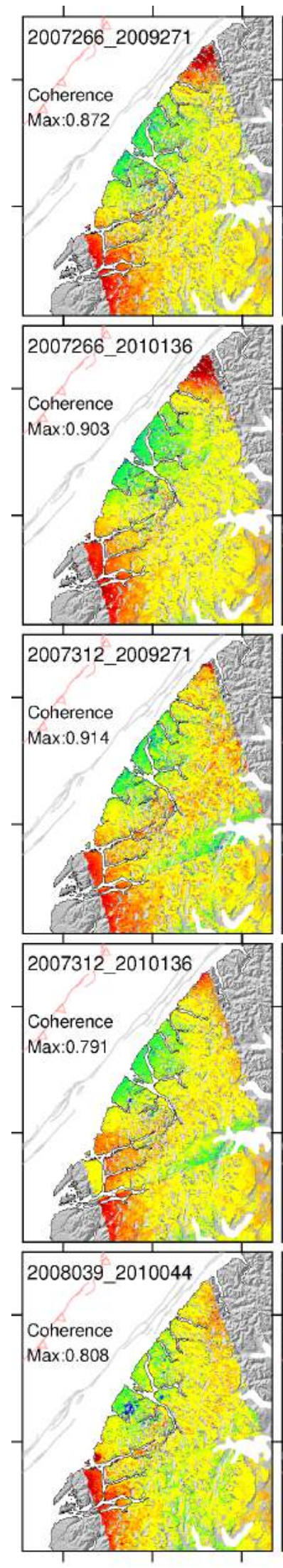
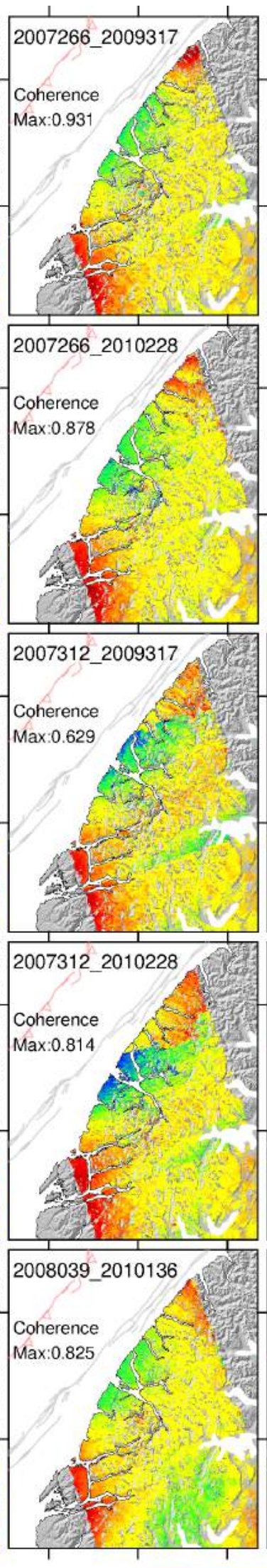
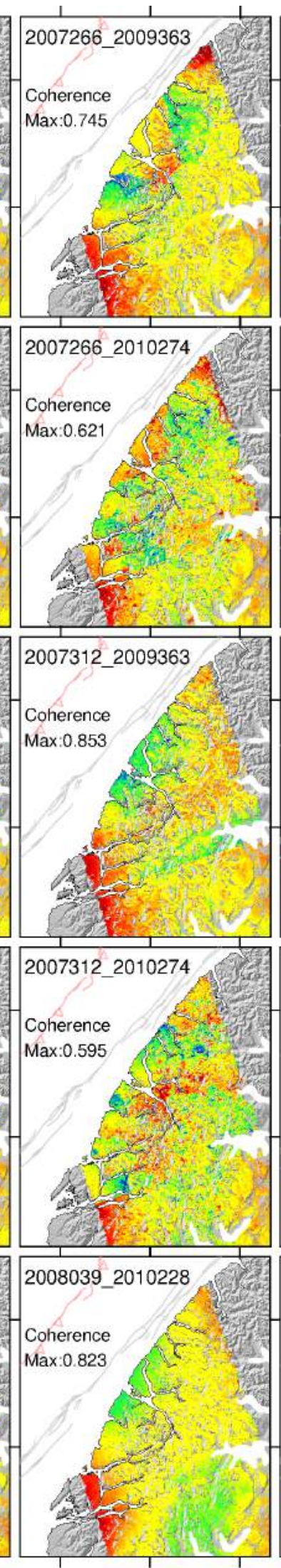

Phase (Radians)
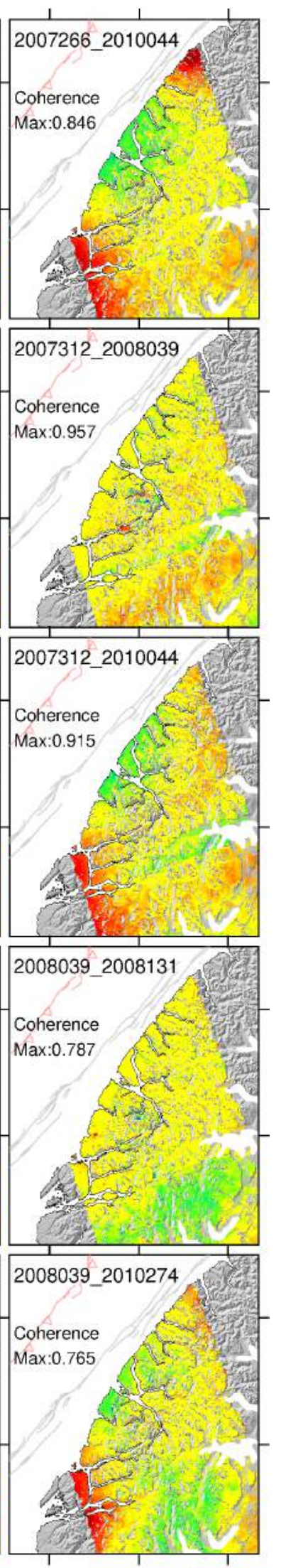

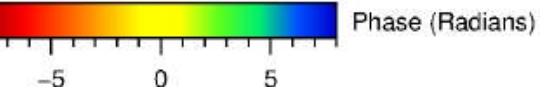

Figure A15: Final Interferograms - Unwrapped Phase 3 

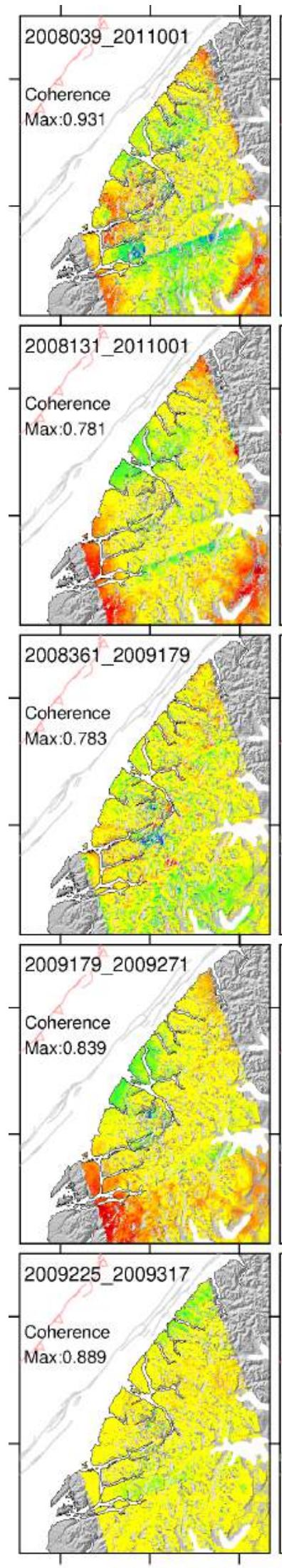
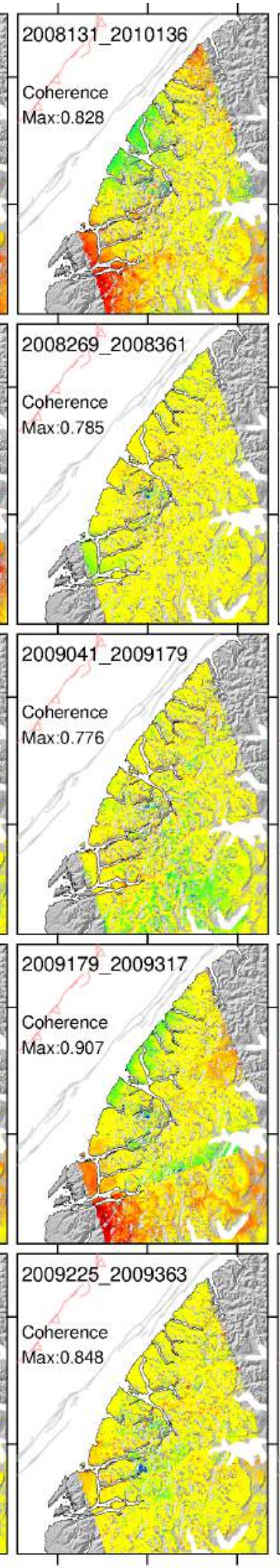
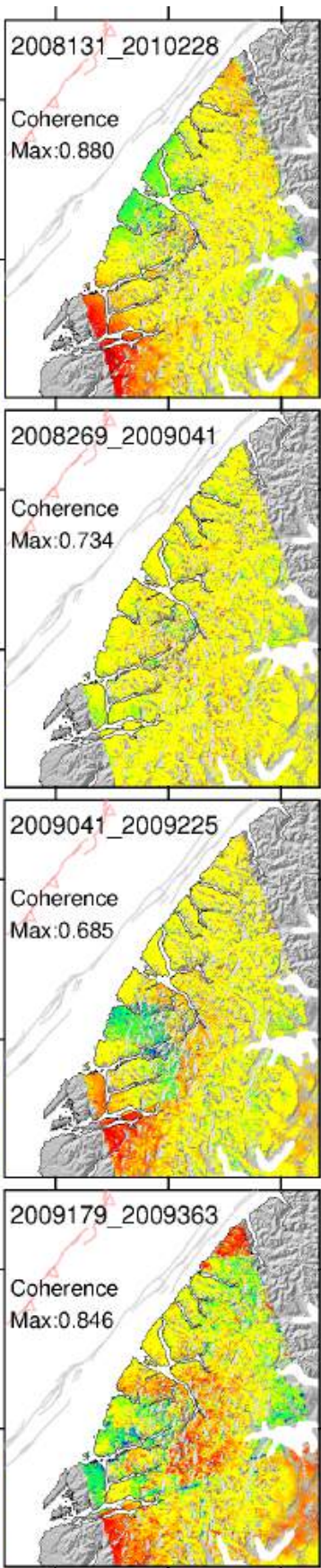

2009225_2010044
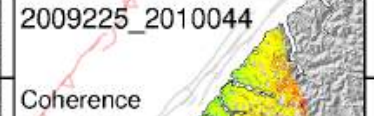

Max:0.881
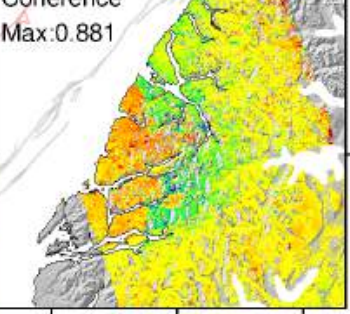

Phase (Radians)
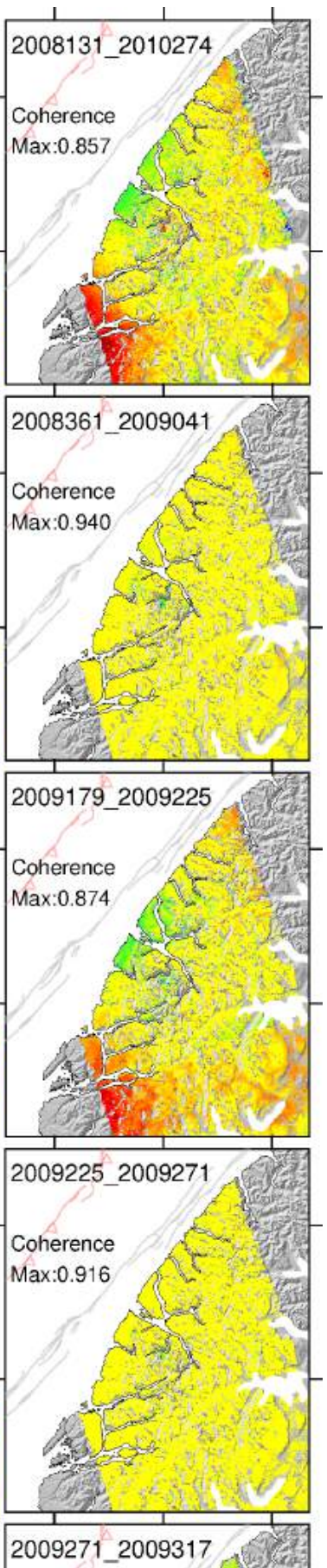

Coherence

Max:0.912

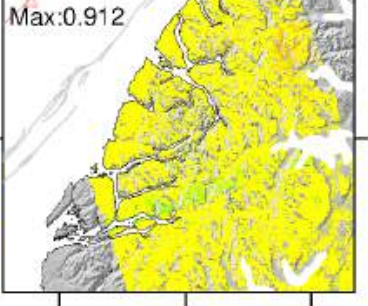

Figure A16: Final Interferograms - Unwrapped Phase 4 

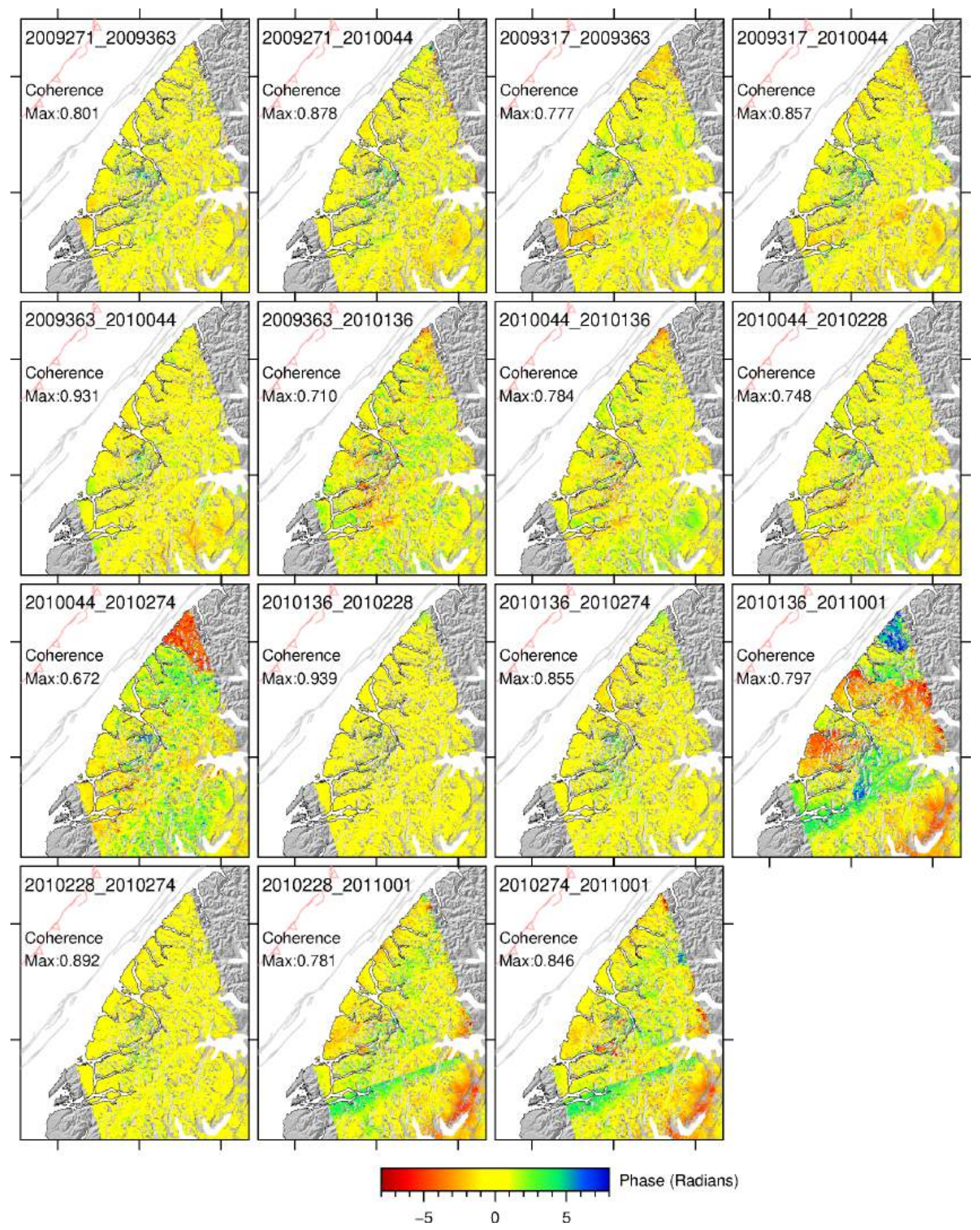

Figure A17: Final Interferograms - Unwrapped Phase 5 